\title{
Entwicklung des Schlafverhaltens im Säuglingsalter
}

Inaugural-Dissertation

zur Erlangung des Grades eines Doktors der Medizin

des Fachbereichs Humanmedizin

der Justus-Liebig-Universität Gießen

vorgelegt von Andreas Heitkamp

aus Remscheid 
Aus dem Medizinischen Zentrum für Psychosomatische Medizin, Abteilung Medizinische Psychologie

Leiterin: Prof. Dr. Renate Deinzer

des Universitätsklinikums Gießen und Marburg GmbH; Standort Gießen

Gutachter: PD Dr. Pauli-Pott

Gutachter: Prof. Dr. Schulz

Tag der Disputation: 10. Juli 2008 


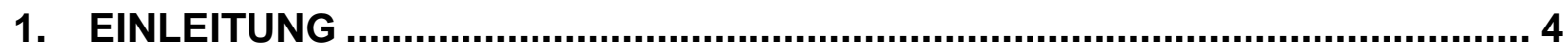

2. LITERATURÜBERSICHT ........................................................................ 6

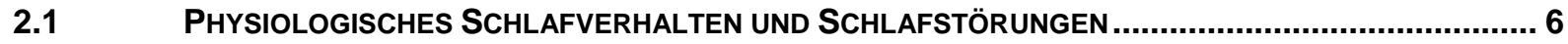

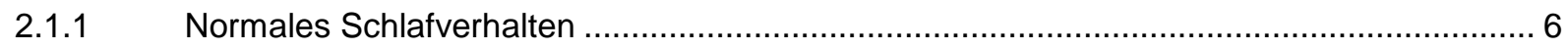

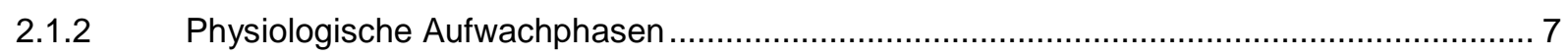

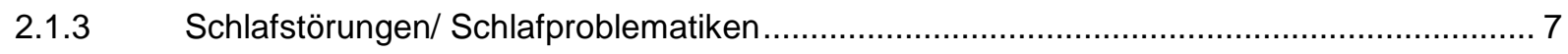

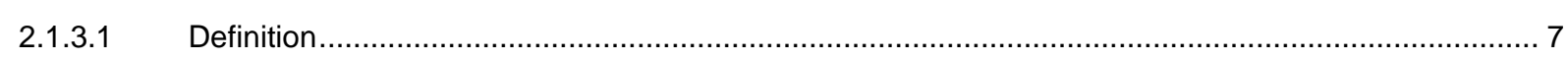

2.1.3.2 Prävalenz und Persistenz von Schlafstörungen.................................................................... 8

2.1.3.3 Veränderungen der Schlafproblematiken im Verlauf der kindlichen Entwicklung ............................. 10

2.1.3.4 Zusammenhang der Schlafprobleme mit elterlichen Merkmalen ................................................... 10

2.1.3.5 Zusammenhang zwischen kindlichem Temperament und Schlafverhalten..................................... 12

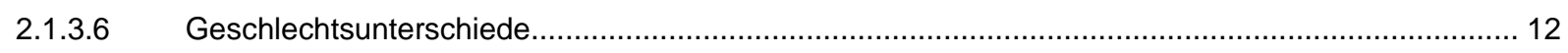

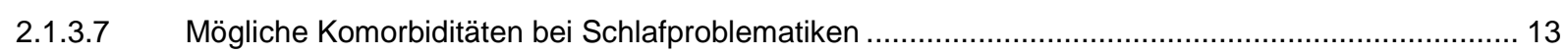

2.1.3.8 Auswirkungen von Schlafstörungen auf die spätere Kindheit .................................................. 13

2.2 DAS RISIKO- UND SCHUTZFAKTORENKONZEPT FÜR KINDLICHE VERHALTENSAUFFÄLLIGKEITEN 15

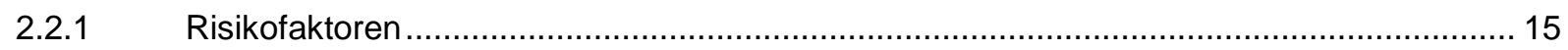

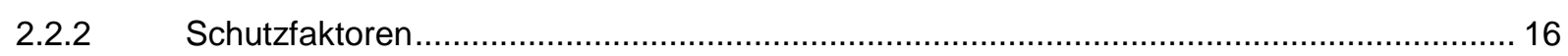

2.2.3 Zusammenwirken von Risiko- und Schutzfaktoren .................................................... 17

2.3 Elterliche Merkmale (Depressivität, ÄNGStLichKeIt, PSYCHISCH/SOZIALE RISIKEN, SOZIO-

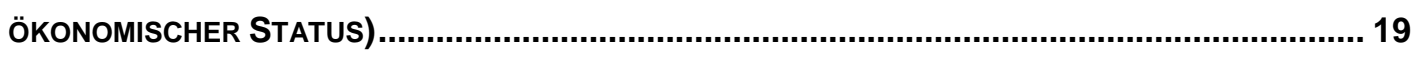

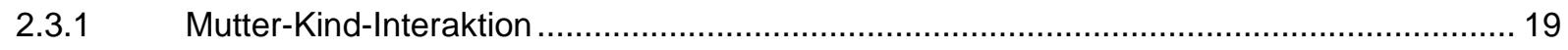

2.3.2 Mütterliche Depressivität und Ängstlichkeit............................................................... 20

2.3.3 Soziale und Sozio-ökonomische Merkmale ............................................................... 20

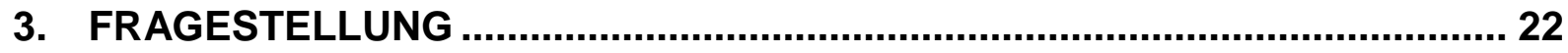

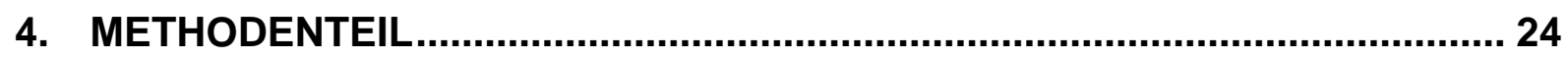

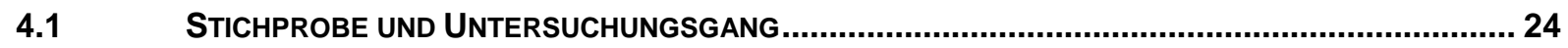

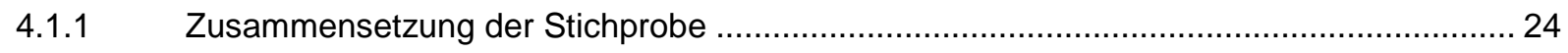

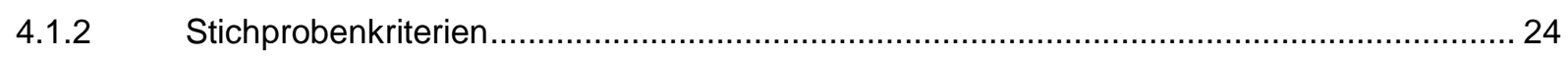

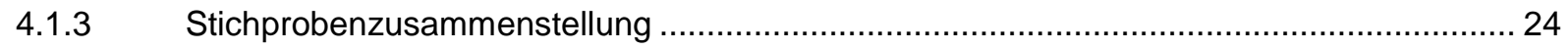

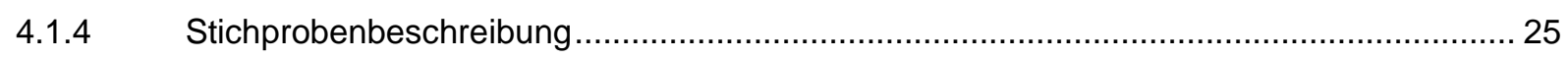

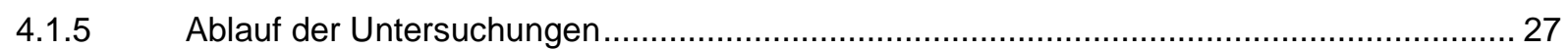

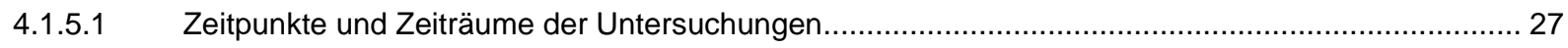

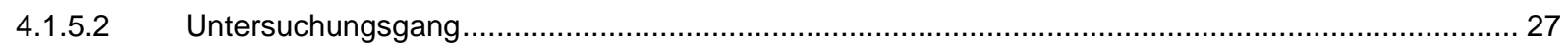


4.2.1 Erfassung des Schlafverhaltens der Säuglinge ........................................................... 30

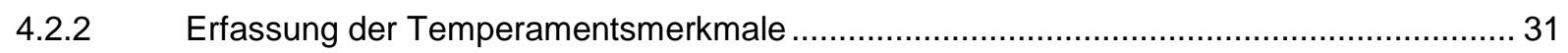

4.2.2.1 Erfassung der Temperamentsmerkmale im Elternurteil .............................................................. 31

4.2.2.2 Erfassung der Temperamentsmerkmale durch Untersuchungen von Reaktionen auf standardisierte

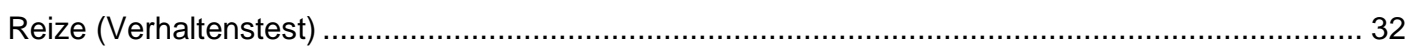

4.2.2.2.1 Erfassung negativer Emotionalität/ Irritierbarkeit im Alter von 4 Monaten....................................... 32

4.2.2.2.2 Erfassung negativer Emotionalität im Alter von 8 und 12 Monaten............................................. 34

4.2.2.2.3 Erfassung von positiver Emotionalität/Soziabilität....................................................................... 37

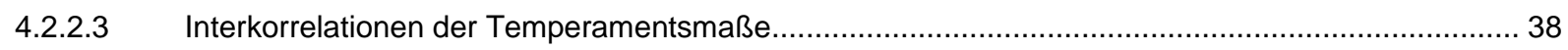

4.2.3 Erfassung der Depressionsneigung und Ängstlichkeit der Bezugsperson ........................ 39

4.2.4 Erfassung des kognitiven Entwicklungsstandes ..................................................... 41

4.2.5 Erfassung von kindlichen Koliken, Stilldauer und Stillverhalten, Körpergewicht und Anzahl

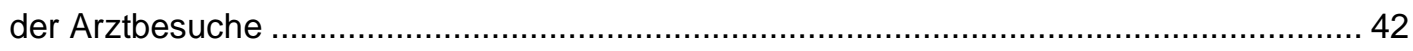

4.2.6 Statistische Signifikanz und statistische Analyseverfahren.............................................. 43

5. ERGEBNISSE

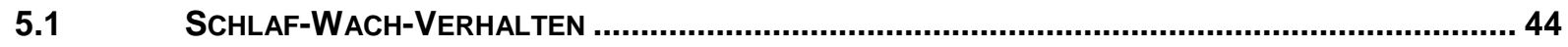

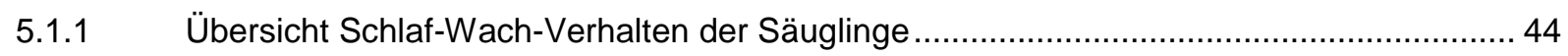

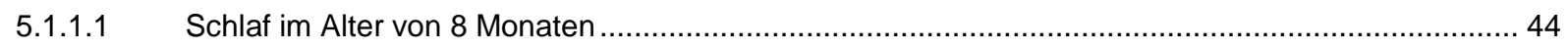

5.1.1.2 Schlafprobleme im Alter von 12 Monaten: Kriteriumsvariablen...................................................... 45

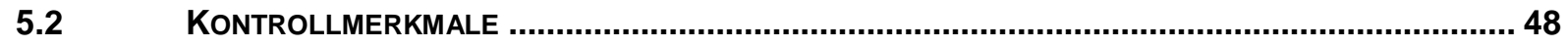

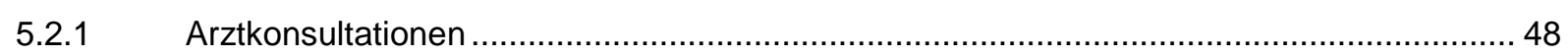

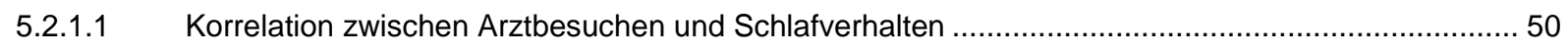

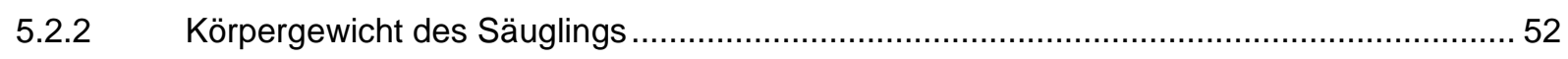

5.2.2.1 Körpergewicht zum Zeitpunkt der Geburt und der U3-, U5- und U6-Untersuchung............................ 53

5.2.2.2 Korrelation des Schlafverhaltens mit dem kindlichen Körpergewicht.............................................. 54

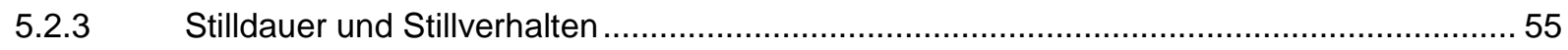

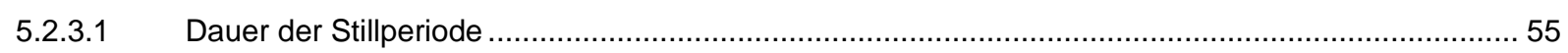

5.2.3.2 Untersuchung der Korrelation zwischen Stilldauer, Gewicht und Schlafeigenschaften .................... 56

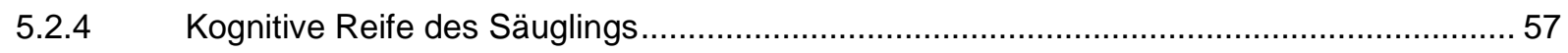

5.2.4.1 Korrelation zwischen kognitiver Entwicklung und Schlafverhalten ................................................ 57

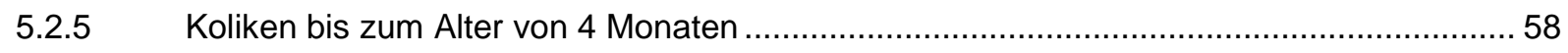

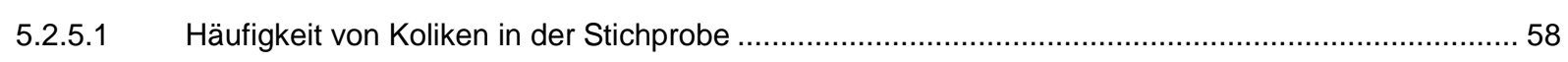

5.2.5.2 Zusammenhänge zwischen Kolikausprägung und Schlafverhalten ............................................. 60

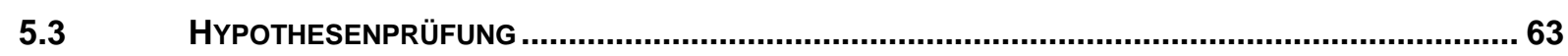

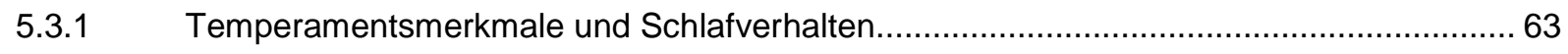

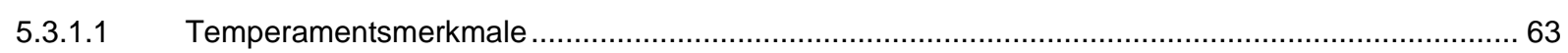

5.3.1.1.1 Untersuchung der Temperamentsmerkmale bezüglich ihrer Interkorrelation...................................63

5.3.1.2 Korrelation zwischen Temperamentsmerkmalen und Schlaf-/ Wachperioden ................................... 65

5.3.1.2.1 Zusammenhänge zwischen positiver Emotionalität und Schlafverhalten.......................................... 66

5.3.1.2.2 Zusammenhänge zwischen negativer Emotionalität und Schlafverhalten ......................................... 68 


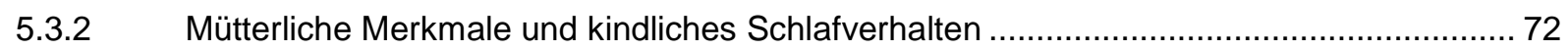

5.3.2.1 Korrelation der mütterlichen Merkmale mit den Aufwachepisoden des Säuglings............................ 72

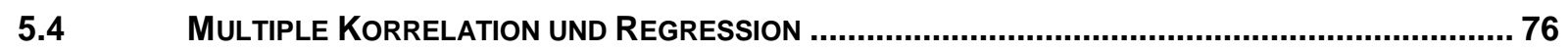

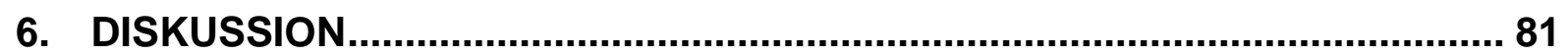

6.1 DAS SCHLAFVERHALTEN

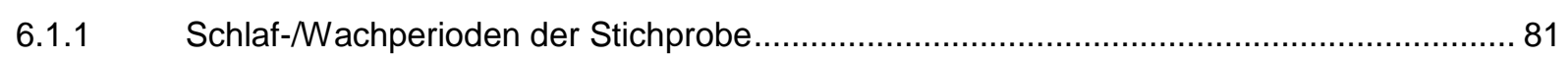

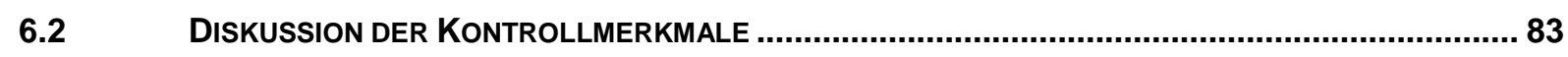

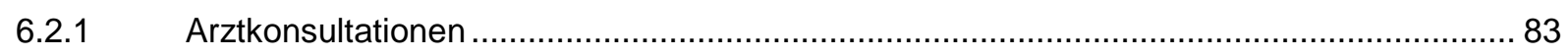

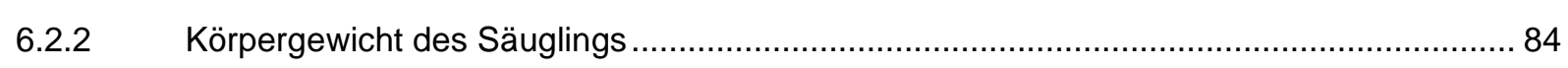

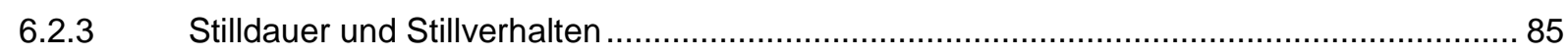

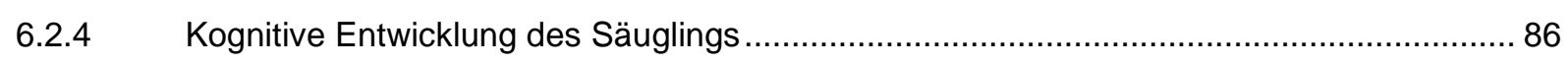

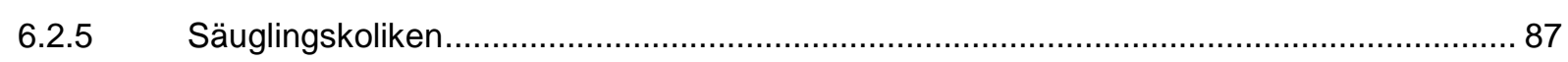

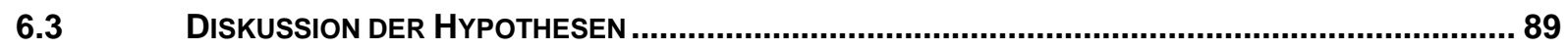

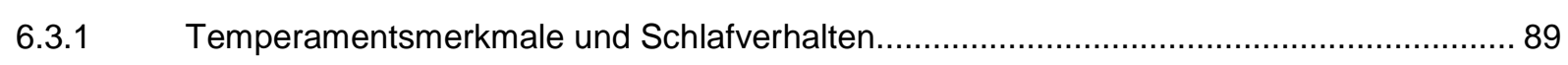

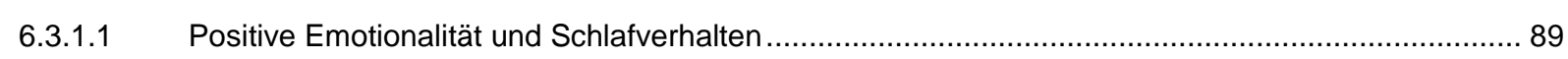

6.3.1.2 Negative Emotionalität und Schlafverhalten .......................................................................... 89

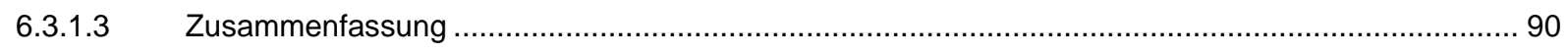

6.3.2 Mütterliche Merkmale und kindliches Schlafverhalten .................................................. 90

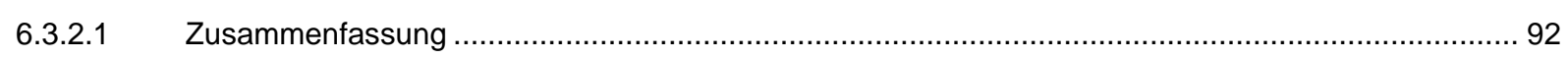

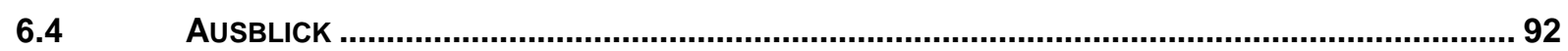

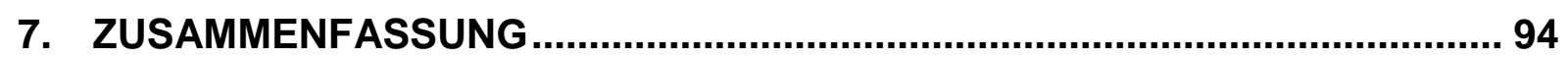

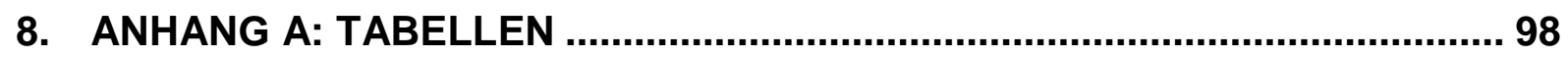

9. ANHANG B: LITERATURVERZEICHNIS ................................................ 124

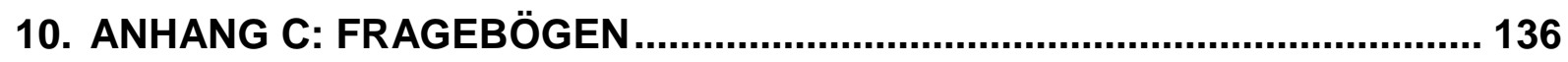

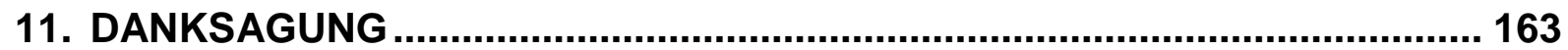

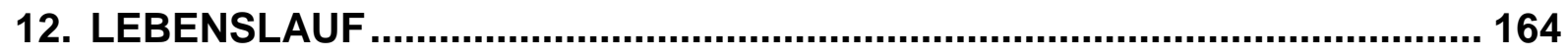




\section{Einleitung}

Über das Vorhandensein von Schlafproblemen im ersten Lebensjahr eines Kindes findet man in der Literatur einen deutlichen Konsens. Versucht man jedoch klare Aussagen über die Entwicklung von Schlafproblemen im Kleinkindalter zu erhalten, begibt man sich in einen Bereich, in dem man mit großer Zurückhaltung konfrontiert wird. Zu dieser These kommen Call und Emde (vgl. Call, 1983; Emde, 1985), welche die Zurückhaltung der Kinder- und Jugendpsychiatrie gegenüber der frühen Entwicklungsperiode unter anderem darin begründet sehen, dass die Unterscheidung zwischen dem, was als normal bzw. als auffällig anzusehen ist, beim Säugling und Kleinkind besondere Schwierigkeiten bereitet (vgl. Emde \& Score, 1984). Hauptmerkmale stellen hier zum einen die inter- und intraindividuelle Variabilität des frühen Verhaltens dar, zum anderen aber auch das Fehlen einschlägiger Kriterien, an denen Beeinträchtigungen beurteilt werden können. Durch die häufige Koinzidenz von körperlichen und seelischen Störungen im Säuglings- und Kleinkindesalter ist eine Trennung in vielen Fällen kaum bzw. nur unter erschwerten Bedingungen möglich. Gerade in dieser frühen Zeit der Entwicklung reagiert der Organismus als körperlichpsychische Einheit und äußert beispielsweise emotionale Beeinträchtigungen in körperlichen Symptomen (Emde, 1987). Wolke et al. (2002) finden einen deutlichen Zusammenhang von persistierenden Regulationsstörungen (Schlafproblematiken, Essverhalten und extremem Schreiverhalten) bis zum Alter von 6 Monaten mit der Entwicklung von Hyperaktivität und mangelnder schulischer Leistungsfähigkeit im weiteren Verlauf der Kindheit. Regulationsstörungen stellen damit einen Risikofaktor für die Entwicklung von Verhaltensauffälligkeiten dar. Weiter gehören die Regulationsstörungen und hier insbesondere die Schlafprobleme zu den häufigsten Vorstellungsgründen von Säuglingen in pädiatrischen Praxen.

Die enge Beziehung zwischen Säugling und Betreuungsperson - in den meisten Fällen die Mutter - erschwert weiter eine Unterscheidung zwischen Störungen des Säuglings und Störungen der Interaktion von Mutter und Kind. Deswegen wird eine Psychopathologie des Säuglings von vielen Autoren abgelehnt, und statt dessen das Konzept der Beziehungsstörung zwischen Eltern und Kind favorisiert (Emde, 1987). Untersuchungen zu dieser Problematik erscheinen aus zwei Gründen relevant: In der Literatur liegen besonders für den Zeitraum der ersten 12 Lebensmonate nur wenige Studien vor, welche sich mit der Entwicklung des Schlafverhaltens sowie der Entste- 
hung von Regulationsstörungen beschäftigen. Zudem wurden bisher im Hinblick auf diese Fragestellung nur selten unausgelesene Stichproben gesunder Mutter-Kind Paare untersucht.

Die zentrale Frage der vorliegenden Arbeit ist daher, unter welchen Bedingungen sich Regulationsstörungen entwickeln und wie sie möglichst frühzeitig erkannt werden können. Hierzu werden im Rahmen dieser Arbeit Merkmale auf kindlicher und mütterlicher Seite hinsichtlich ihres Vorhersagewertes für kindliches Schlafverhalten im 12. Lebensmonat zu drei vorgegebenen Untersuchungszeitpunkten (im 4., 8. und 12. Monat) geprüft. Vorrangig werden Beziehungen zwischen kindlichem Temperament und mütterlicher Depressionsneigung/Ängstlichkeit mit möglichen Schlafproblematiken am Ende des ersten Lebensjahres untersucht. Die hierzu verwendeten Daten wurden im Rahmen der Längsschnittstudie zu Temperamentsentwicklung (Leitung: PD Dr. U. Pauli-Pott; DFG Kennziffer: Pa 543/2-1,2,3,4) der Gießener Abteilung Medizinische Psychologie gewonnen.

Die Ergebnisse könnten dazu beitragen, die Qualität der pädiatrischen Beratung und Indikationsentscheidungen für eine psychotherapeutische Unterstützung zu verbessern.

Im nachfolgenden Kapitel soll zunächst auf das physiologische Schlafverhalten sowie auf mögliche pathologische Veränderungen in dessen Entwicklung eingegangen werden. Im weiteren Verlauf sollen Konzepte von Risiko- und Schutzfaktoren des Kindes im Hinblick auf sich entwickelnde Verhaltensauffälligkeiten erläutert werden. Am Ende des folgenden Kapitels werden elterliche Merkmale wie Depressivität, Ängstlichkeit, psychisch/soziale Risiken und der sozio-ökonomische Status in Beziehung zur kindlichen Entwicklung betrachtet. 


\section{Literaturübersicht}

\subsection{Physiologisches Schlafverhalten und Schlafstörungen}

\subsubsection{Normales Schlafverhalten}

Um die Problematik und das Ausmaß der Schlafstörungen richtig beurteilen zu können, ist es wichtig, einen Überblick über das normale Schlafverhalten und die normale Schlafentwicklung zu geben. Wolke (1994) berichtet von einer großen Veränderung in der physiologischen Schlaforganisation, dem zirkadischen (Tag-Nacht) und ultradiaschen (innerhalb des Tages) Rhythmus sowie der Schlafdauer innerhalb des ersten Lebensjahres. Bereits in den ersten Lebenswochen beginnt die zirkadische Schlaf-Wach-Organisation, wobei die Platzierung der längsten Schlafperiode in der Nacht auffällt. In der ersten Hälfte des ersten Lebensjahres vollzieht sich die Differenzierung in aktivem (REM), leichtem (NREM 1,2) und tiefem Schlaf (NREM 3,4). Beginnt das Neugeborene seinen Schlaf noch mit der REM-Schlaf-Phase, vollzieht sich das Einschlafen ab dem 4. bis 6. Monat bis zum Lebensende im NREM Schlaf (Wolke, 1994). Sadeh (1996) und Hoppenbrouwers et al. (1988) bestätigen mit ihren Ergebnissen, dass Neugeborene über $60 \%$ des Tages schlafen und über die Hälfte ihres Schlafes im REM-Schlaf bzw. im aktivem Schlaf verbringen (Sadeh et al., 1996; Hoppenbrouwers et al., 1988). Im Alter von 6 bis 8 Monaten ist der Schlaf des Säuglings hinsichtlich der REM-Schlafphase dem des Erwachsenen sehr ähnlich, wobei sich die Schlafphasen der Kleinkinder häufiger über den Tag verteilen. Die Schlafdauer verkürzt sich vom Neugeborenenalter mit 14 bis 16 Stunden auf ca. 12 bis 13 Stunden beim Zweijährigen (Wolke, 1994). Weiterhin zeigt sich nun beim zweijährigen Kind ein Rückgang des aktiven Schlafes bzw. REM-Schlafes auf ungefähr $35 \%$ des Gesamtschlafes (Anders et al., 2000). 


\subsubsection{Physiologische Aufwachphasen}

In Bezug auf das Schlafverhalten der Kleinkinder ist es interessant zu wissen, dass das Problem des nächtlichen Aufwachens keine Belastung für die Kinder darstellt, für die Erwachsenen jedoch anstrengend ist. Im Durchschnitt weist ein Kleinkind zwischen drei und sieben Aufwachperioden pro Nacht auf, was videosomnographische Aufnahmen bestätigen. Diese Unterbrechung des kindlichen Schlafes rückt jedoch erst dann in die Aufmerksamkeit der Eltern bzw. stellt eine Belastung für diese dar, wenn sich das Kind durch Schreien bemerkbar macht, die Eltern weckt oder beruhigt werden muss (Minde et al., 1993; Gaylor et al., 1998). Kinder sind nicht mit der Fähigkeit geboren die Nacht durchzuschlafen. Häufiges Aufwachen ist vielmehr adaptiv zur häufigen Nahrungsaufnahme und zum Wachstum in den ersten 6 Lebensmonaten (Wolke et al., 1998; Skuse et al., 1994).

\subsubsection{Schlafstörungen/ Schlafproblematiken}

\subsubsection{Definition}

Hauptaugenmerk dieser Arbeit sind die Durchschlafprobleme des Säuglings, welche neben den Einschlafproblemen in die Gruppe der Insomnien gehören (Dilling et al. 1991; Saß et al. 1996). Diese Gruppe stellt zugleich die häufigste Schlafstörung des ersten Lebensjahres dar (Wolke, 1999).

Es zeigt sich, dass das generelle nächtliche Aufwachen nicht uneingeschränkt als Schlafstörung bzw. Schlafproblematik anzusehen ist. Vielmehr leidet jenes Kind an einer Problematik, welches eigenständig nicht in der Lage ist, sich zu beruhigen und selbständig seinen Schlaf fortzusetzen (Minde et al., 1993; Gaylor et al.,1998).

Wolke (1999) unterscheidet bei den Durchschlafproblemen des Weiteren zwischen zwei Untergruppen. Zur ersten Gruppe gehören die Kinder, die älter als 6 Monate sind und an 5 Nächten pro Woche mindestens einmal pro Nacht zwischen 0:00 und 5:00 Uhr aufwachen. Zur zweiten Gruppe gehören Kinder mit schweren Durchschlafproblemen, nämlich solche, die mehrmals pro Nacht aufwachen (Wolke, 1999). Die Deutsche Gesellschaft für Kinder- und Jugendpsychiatrie definiert Durchschlafstörungen als durchschnittlich mehr als dreimal nächtliches Aufwachen in mindestens 4 
Nächten der Woche verbunden mit der Unfähigkeit, ohne elterliche Hilfen alleine wieder einzuschlafen (Deutsche Gesellschaft für Kinder- und Jugendpsychiatrie, 2003).

\subsubsection{Prävalenz und Persistenz von Schlafstörungen}

Das Auftreten von Schlafstörungen in der kindlichen Entwicklung ist unumstritten, jedoch schwanken die Angaben der Häufigkeiten von Autor zu Autor. Wolke, Meyer, Ohrt und Riegel (1995) fanden in der Bayrischen Entwicklungsstudie, in der über 1000 reifgeborene Kinder nachuntersucht wurden, Schlafstörungen bei 21,5\% der Kinder im Alter von 5 Monaten, bei 21,8 \% im Alter von 20 Monaten und bei 13,3\% im Alter von viereinhalb Jahren. Fegert et al. (1997) untersuchten 1314 Kinder in den ersten drei Lebensjahren. Etwa $20 \%$ der Kinder weckten die Eltern mehrmals, weitere 20 \% einmal pro Nacht auf. 15,2 \% der einjährigen- und 4,7\% der zweijährigen Kinder brauchten längere Zeit, um wieder einzuschlafen. In beiden epidemiologischen Längsschnittstudien erwiesen sich die Schlafstörungen als relativ stabil. Mindestens $30 \%$ der Kinder, die mit fünf Monaten regelmäßig aufwachen, neigen auch noch mit 20 Monaten oder 3 Jahren dazu.

So zeigen Gaylor et al. (2001) in ihrer Studie, in der 33 Kinder im Alter von 12 und 39 Monaten hinsichtlich ihres Schlafverhaltens untersucht wurden, ebenfalls ein Anteil von 33\% der Kinder mit Schlafstörungen. Zu beiden Untersuchungszeitpunkten lag die spontane Aufwachrate der Kinder bei circa drei Episoden pro Nacht, wobei diese mittels Videoaufnahmen ermittelt wurden. Demgegenüber beschreiben Hiscock et al. (2001) in ihrer Studie mit 738 Mutter-Kind Paaren 46\% der 6-12 Monate alten Säuglinge als schlafgestört, wobei die Aufwachrate pro Nacht bei diesen schlafgestörten Kindern im Vergleich zur vorherigen Studie lediglich bei zwei Ereignissen pro Nacht liegt. Ausschlaggebend ist hierbei jedoch die Art der Erfassung der Aufwachphasen. Wurde in der ersten Untersuchung das Aufwachen videographisch erfasst, stand in der Studie von Hiscock et al. die Aussage der Mutter im Vordergrund. Dabei ist erwähnenswert, dass die Mutter lediglich über die Aufwachzeitpunkte berichten kann, in denen sich ihr Kind aktiv bemerkbar gemacht hat und nicht selbständig wieder in den Schlaf fand. Über ein ähnliches Phänomen berichtet Sadeh (1996) in seiner Studie, in welcher er 66 Säuglinge auf ihre Aufwachhäufigkeit pro Nacht zum einen mit- 
tels objektivem Monitoring und andererseits mittels Tagebuch der Eltern untersucht. Es zeigte sich eine generelle Überbewertung der kindlichen Schlafdauer und eine generelle Unterbewertung der Häufigkeit der Aufwachepisoden pro Nacht mittels des Elterntagebuches im Vergleich zum objektivem Monitoring.

Zuckerman et al. (1987) ermittelten hingegen in ihren Studien mit 308 Mutter-KindPaaren eine geringere Häufigkeit an Schlafproblematiken (10 \%) im Alter von 8 Monaten. Die Mütter berichteten jedoch über ein wesentlich häufigeres Aufwachen der Kinder pro Nacht (drei oder mehrere Male) als in den vorher erwähnten Studien. Die relativ niedrige Aufwachhäufigkeit lässt sich damit erklären, dass den Mütter in der Studie drei Antwortmöglichkeiten hinsichtlich der Schlafproblematik des Kindes zur Verfügung standen: „das Baby wacht 3 oder mehr Mal pro Nacht auf“, „das Kind benötigt nach dem Aufwachen mehr als eine Stunde um sich wieder zu beruhigen“ und „der mütterliche Schlaf wird aufgrund des Kindes häufig unterbrochen“. Insgesamt gaben jedoch $18 \%$ der Mütter mindestens eine der genannten Antworten.

In einer umfangreichen Studie mit 2518 schwedischen Kindern im Alter von 6 bis 18 Monaten berichteten Thunström et al. (1999) bei knapp der Hälfte aller Kinder $(48,3 \%)$ über in der Vergangenheit aufgetretene Schlafprobleme. Weiter berichteten $16 \%$ der Eltern über mäßige bis schwere Probleme beim Einschlafen ihrer Kinder und $30 \%$ klagten über häufiges nächtliches Aufwachen ihres Nachwuchses. Als Ursache beschrieben Thunström et al. in besonderem Maße übermäßiges elterliches Umsorgen des Kindes während des Abends und der Nacht in Form von Füttern, Singen oder Wiegen, auf das in Abschnitt 2.1.3.4 näher eingegangen werden soll.

Nicht selten bleibt das Schlafproblem bei 50 \% der vorbelasteten Kinder für mehr als 5 Jahre bestehen und führt zu späteren psychologischen Problemen (Richman et al. 1982; Pollock 1992).

Zusammenfassend wird deutlich, dass sich das Schlafverhalten des Kindes in den ersten Jahren als ein relativ häufiges Problem darstellt. Die Studienergebnisse zur Prävalenz schwanken jedoch wegen Differenzen in den verwendeten Definitionen und Erfassungsmethoden. 


\subsubsection{Veränderungen der Schlafproblematiken im Verlauf der kindlichen Entwicklung}

Das erstmalige Auftreten von Schlafstörungen zu einem bestimmten Zeitpunkt des ersten Lebensjahres war Bestandteil einiger Studien.

Eine Untersuchung von 48 gesunden Säuglingen im Alter von 1-12 Monaten (Ficca et al., 1999) zeigte, dass insbesondere das zweite Lebenshalbjahr für die Entwicklung des Schlaf-Wach Rhythmus eine wichtige Rolle spielt. So lagen die nächtlichen Aufwachepisoden bei Kindern bis zum Alter von 6 Monaten bei durchschnittlich 7,5 Episoden pro Nacht, im zweiten Lebenshalbjahr reduzierten sie sich auf nur noch 4,9 Episoden pro Nacht.

So zeigt sich, dass mit dem Rückgang der REM Schlafphasen (aktiver Schlaf), der beim Neugeborenen mehr als 60 \% des Schlafes ausmacht (Sadeh et al., 1996; Hoppenbrouwers et al., 1988) und aus welchem der Säugling nachweislich öfters erwacht (Ficca et al., 1999), die Abnahme der nächtlichen Aufwachepisoden im ersten Jahr begründet werden kann.

Dies bestätigen weitere Studien von Hoppenbrouwers et al. (1988) und Louis et al. (1997), die bei der Untersuchung von jeweils 20 bzw. 15 Kindern im Zeitraum des ersten Lebensjahres über eine Abnahme der aktiven Schlafphase bzw. REM Phase und einer Zunahme der ruhigen Schlafphase (NREM) berichten. Dementsprechend zeigten die Säuglinge beider Studien einen Rückgang der nächtlichen Aufwachepisoden im zweiten Lebenshalbjahr.

\subsubsection{Zusammenhang der Schlafprobleme mit elterlichen Merkmalen}

Die im Jahre 1999 durchgeführte Untersuchung von Thunström et al. (1999) an 2518 Kindern im Alter von 6 bis 18 Monaten zeigte verschiedene Merkmale mit Beziehung zu den Schlafproblemen auf. Als entscheidendes Merkmal zeigte sich der deutliche Zusammenhang des Schlafverhaltens mit dem elterlichen Umsorgen (Füttern, Singen, Wiegen) in den Abend- und Nachtstunden sowie die Sorgen und Ängste der Eltern um das Wohl des Kindes. So wachten jene Kinder häufiger auf, welche vor dem Schlafengehen intensiv umsorgt wurden und deren Eltern Angst um das gesundheitliches Wohlergehen ihres Kindes hatten. Weiter erwies sich der Zeitraum 
vom 8. bis zum 10. Lebensmonat als Periode, in der besonders häufig Schlafprobleme auftraten.

Auch Burnham et al. (2002) zeigten an 80 Kindern im ersten Lebensjahr, dass jene Kinder besser schliefen, deren Eltern nicht direkt zum Kind eilten, wenn dieses sich durch das Aufwachen bemerkbar machte. Weiterhin wurde anhand dieser Studie deutlich, dass Kinder, die wach ins Bett gelegt wurden sowohl besser einschlafen als auch nach dem nächtlichen Aufwachen die Fähigkeit besaßen, ohne elterliche Hilfe wieder in den Schlaf zurückzufinden. Die neuronale Reife des Kindes, welche in enger Beziehung zur Dauer des kindlichen NREM-Schlafes nach der Geburt gesetzt wurde, erwies sich in dieser Studie ebenfalls als wichtige Größe. So zeigt ein Kind mit langen und früh einsetzenden NREM-Schlafphasen ein größeres Maß an Reife und damit verbunden eine erhöhte Fähigkeit an Selbstregulation mit 12 Monaten.

In einer 2002 durchgeführten Studie von Hiscock et al. (2002), bei der 156 Mütter von Kindern im Alter von 6 bis 12 Monaten mit Schlafschwierigkeiten untersucht wurden, ergaben sich Zusammenhänge der Schlafprobleme mit mütterlichen Depressionen und der Art und Weise der Fütterung der Kinder (Brust- oder Flaschennahrung). Bei Müttern, welche ihre Kinder stillten, fanden sich bei $56 \%$ der Kinder Schlafschwierigkeiten im Gegensatz zu Kindern mit Flaschennahrung (40\%). Weiter zeigte sich in dieser Studie ein niedriger Sozialstatus mit kindlichen Schlafschwierigkeiten verknüpft. Ähnliche Ergebnisse zeigte ein Vergleich von 1057 Kindern in Finnland mit 4427 Kindern in Deutschland, der von Wolke et al. (1998) durchgeführt wurde. Hier ergab sich, dass im Alter von 5 Monaten deutlich mehr finnische als deutsche Kinder Schlafschwierigkeiten zeigten (finnische: 25,5\% - 48\%; deutsche 15,1\% - 19,1\%), was von Wolke et al. (1998) mit einer erhöhten Rate an Bruststillen erklärt wurde. Erstaunlicherweise zeigte das Bruststillen in Finnland nur im 5. Lebensmonat einen Zusammenhang zum Schlafverhalten. Nach dieser Zeit schliefen die finnischen Kinder nahezu normal im Gegensatz zu den deutschen Kindern, welche auch in der Folgezeit häufiger aufwachten.

Auch über einen Studienzeitraum von fünfeinhalb Jahren lagen bei Kindern mit chronischen Schlafstörungen ähnliche Merkmale vor (Thunström, 2002). So enthielt die Gruppe der schlafgestörten Kinder ( $n=27$ ) im Gegensatz zur Kontrollgruppe vermehrt brustgestillte Kinder und solche, die nachts gefüttert wurden oder bei den Eltern im Bett schliefen. Weiterhin fanden sich in den betroffenen Familien gehäuft psychoso- 
ziale oder finanzielle Probleme, mütterliche Depressionen, elterlicher Stress und gesundheitliche Beeinträchtigungen.

\subsubsection{Zusammenhang zwischen kindlichem Temperament und Schlafverhalten}

Scher et al. (1998) untersuchten 1998 insgesamt 30 Kinder im Alter von 12 Monaten in Bezug auf Temperamentsmerkmale und inr Schlafverhalten. Bei Kindern, die von ihren Müttern mit Hilfe des "Carey Toddler Temperament Questionnaire" (TTQ; von Carey \& McDevitt, 1978) als rhythmisch und vorhersehbar eingestuft wurden, zeigte sich ein frühzeitigeres Zubettgehen und eine längere Schlafdauer als bei den „unrhythmischen“ Kindern. Die „rhythmischen“ Kinder zeigten jedoch im Vergleich zu den „unrhythmischen“ Kindern, welche zwar später zu Bett gebracht wurden und demzufolge eine kürzere Schlafdauer aufwiesen, ein Schlafverhalten mit häufigerem Aufwachen und niedrigerer Effektivität.

\subsubsection{Geschlechtsunterschiede}

Im Alter bis zu 12 Monaten zeigen sich keine signifikanten Unterschiede in den Schlafeigenschaften bzw. Schlafstörungen zwischen beiden Geschlechtern.

Dies belegen verschiedene Studien, welche keinen nachweisbaren Zusammenhang zwischen Geschlecht oder Geburtsverlauf und dem Schlafverhalten des Kindes aufzeigen konnten (Scher, 2001, Burnham et al., 2002, Fegert et al., 1997). Lediglich bei den 2518 Kindern, die von Thunström (1999) auf ihre Schlafeigenschaften untersucht wurden, waren es geringfügig mehr Jungen, die ernstere Schlafprobleme aufwiesen. Dieses Ergebnis sollte nach Thunströms Aussagen im Hinblick auf vorangegangene Studien zunächst einmal als vorläufig angesehen werden und durch zukünftige Untersuchungen überprüft werden. 


\subsubsection{Mögliche Komorbiditäten bei Schlafproblematiken}

Eine bedeutende Assoziation in Bezug auf kindliche Schlafproblematiken zeigen Schrei- und Fütterungsprobleme.

Sowohl Wolke et al. (1994) als auch v. Hofacker et al. (1996) belegen dies anhand von Untersuchungen. Ihre Stichproben setzten sich aus Kindern zusammen, welche im Alter von 1 bis 6 Monaten aufgrund exzessiven Schreiens (circa 4,5 bzw. 6 Stunden pro Tag) vorgestellt wurden. V. Hofacker und Papousek (1998) wiesen in ihrer Stichprobe nach, dass 77\% der Schreikinder gleichzeitig ein Schlafproblem besaßen; bei $34 \%$ der Kinder bestand neben dem exzessiven Schreien auch noch ein Fütterungsproblem. Ähnliche Ergebnisse zeigen Wolke et al. (1994) in ihren Untersuchungen: 80 \% der Schreikinder hatten gleichzeitig auch ein Fütterungsproblem, $70 \%$ der Schreikinder hatten nebenbei auch ein Schlafproblem.

\subsubsection{Auswirkungen von Schlafstörungen auf die spätere Kindheit}

Einige Studien beschäftigen sich mit möglichen Entwicklungsauffälligkeiten im späteren Kindheitsverlauf bei Säuglingen mit Schlafproblematiken. Bei einer Längsschnittstudie mit 308 Kindern, welche im Zeitraum vom 8. Lebensmonat bis zum 3. Lebensjahr gemacht wurde, stellte sich in der Gruppe der Kinder mit fortbestehenden Schlafstörungen heraus, dass diese bevorzugt Verhaltensproblematiken -insbesondere Gemüts- und Verhaltensregulationsstörungen- aufwiesen, im Gegensatz zu der Vergleichsgruppe, bei der keine Schlafstörungen bestanden (Zuckerman et al. (1987). Zu ähnlichen Erkenntnissen kommen Minde et al. (1993) in ihren Untersuchungen von 28 schlafgestörten Kindern im Vergleich zu 30 Kontroll-Kindern im Alter von 12 bis 36 Monaten. Hierbei zeigte sich in der Gruppe der schlecht schlafenden Kinder ein häufigeres Auftreten von Verhaltensproblemen und schwierigerem Temperament. Andere Autoren sehen kindliche Schreiproblematiken und damit verbundene Schlaf- und Essensprobleme als Vorläufer für Verhaltensregulationsstörungen wie Hyperaktivität und Verhaltensprobleme in späterer Kindheit (Wolke et al.,1995; DeGangi et al., 1991; Wolke, 1999; Moffitt, 1996). Wolke (2002) beschreibt indes einen deutlichen Zusammenhang von Schrei- und Regulationsproblemen in früher Kindheit mit vermehrten Problemen in späterer Kindheit wie Hyperaktivität, 
Betragensstörungen, negativer Emotionalität sowie einer mangelnden Anpassungsfähigkeit im Vergleich zu Kindern aus der Kontrollgruppe. Wolke (2002) vertritt sogar die These, dass Kinder mit frühen Verhaltensproblemen eine geringere akademische Leistungsfähigkeit im späteren Leben haben werden.

So zeigt auch die Beobachtung von schwedischen Kindern (Thunström, 2002) über fünfeinhalb Jahre einen merklichen Zusammenhang zwischen frühkindlichen Schlafstörungen bzw. Aufmerksamkeitsdefiziten und Hyperaktivität im Alter von $51 / 2$ Jahren. So wiesen 7 Kinder aus der Gruppe mit Schlafstörungen $(n=27)$ im fortgeschrittenen Alter Verhaltensstörungen auf im Gegensatz zur Kontrollgruppe $(n=27)$, in der kein Kind eine Verhaltensstörung hatte.

Regulationsstörungen stellen Risikofaktoren für die Entwicklung späterer Verhaltensauffälligkeiten dar. In besonderem Maße sind jene Kinder gefährdet, bei denen die Störungen über das erste Lebenshalbjahr hinaus persistieren. Im Folgenden erfolgt die Darstellung derzeit aktueller Risiko- und Schutzfaktorenkonzepte. 


\subsection{Das Risiko- und Schutzfaktorenkonzept für kindliche Verhal- tensauffälligkeiten}

Bei den Risiko- und Schutzfaktoren unterscheidet man endogene, kindbezogene Faktoren von exogenen, umgebungsbezogenen Faktoren.

\subsubsection{Risikofaktoren}

Endogene Risikofaktoren betreffen kindbezogene Bedingungen. Hierbei spricht man häufig von biologischen Risikofaktoren oder auch Vulnerabilitätsfaktoren. Diese umfassen auf der einen Seite genetische Dispositionen und chronische Krankheiten, andererseits Merkmale wie niedrige Intelligenz, vermehrte Ablenkbarkeit oder ein schwieriges Temperament. Faktoren, welche das Kind von Geburt an aufweist, werden als primäre Vulnerabilität bezeichnet. Demgegenüber entsteht die sekundäre Vulnerabilität in der Auseinandersetzung des Kindes mit seiner Umwelt (Scheithauer et al., 2000).

Exogene Faktoren stellen Bedingungen dar, welche psychosoziale Merkmale der Umwelt des Individuums betreffen. Diese, auch als Stressoren beschriebenen Merkmale umfassen familiäre oder soziale Faktoren, wie materielle Notlage, Kriminalität eines Elternteils oder chronische Disharmonie in der Familie (Rutter, 1988; Jensen et al., 1990). Familiäre Lebensbedingungen wie beengte Wohnverhältnisse, mangelnde soziale Integration und chronische Schwierigkeiten fließen des Weiteren in die Risikofaktoren ein (Laucht et al., 1998).

Cicchetti, Rogosch und Toth (1997) gehen davon aus, dass Risikofaktoren nicht unmittelbar mit Entwicklungsrisiken verbunden sein müssen. Ein mitentscheidender Faktor ist die gleichzeitig vorhandene Vulnerabilität des Kindes (Cicchetti et al., 1997; Cicchetti \& Toth 1997).

Endogene und exogene Risiken interagieren also sehr wahrscheinlich miteinander im Hinblick auf die weitere Entwicklung des Kindes.

Sehr wahrscheinlich spielen der Zeitpunkt des Auftretens der Risikobedingung innerhalb bestimmter Entwicklungsphasen, die Dauer des Einwirkens sowie die Intensität des Faktors eine entscheidende Rolle (Kazdin et al., 1997). Laucht et al. (1996) zeig- 
ten in ihrer Untersuchung an 384 Kindern (darunter 210 Hochrisikokinder), welche von der Geburt bis ins Schulalter begleitet wurden, dass es meist zu einer Addition des Einflusses biologischer und psychosozialer Risikobedingungen kommt. Moffitt (1993) sieht neurophysiologische Fehlentwicklungen, welche durch vor- bzw. nachgeburtliche Ereignisse entstanden sein können, als Ursachen für Verhaltensstörungen, Hyperaktivität, Aggressivität und Entwicklungsdefizite im kindlichen Leben. Petermann und Scheithauer (Petermann \& Scheithauer, 1998; Scheithauer \& Petermann, 2000) gehen davon aus, dass diese Defizite in der weiteren Entwicklung des Kindes mit schlechten Schulleistungen und daraus resultierendem mangelndem Selbstwertgefühl und aggressivem Verhalten verknüpft sind.

\subsubsection{Schutzfaktoren}

In verschiedenen Studien konnte nachgewiesen werden, dass Kinder, die unter massiven psychischen Belastungen und widrigsten Lebensumständen aufwuchsen, sich dennoch zu gesunden Erwachsenen entwickelten. So zeigten sich Schutzfaktoren, welche die Kinder vor den Auswirkungen der Risikofaktoren bewahrt hatten. Diese werden in personale, kindbezogene-, Resilienzfaktoren und soziale, umgebungsbezogene Faktoren (innerhalb der Familie und im weiteren Umfeld) unterteilt (Laucht et al., 1997; Rutter, 1985). Weiterhin zeigte sich in einer Untersuchung von Newcomb und Felix-Ortiz (1992), dass es bei den Schutzfaktoren die Möglichkeit der gegenseitigen Potenzierung gibt. Das heißt: Wenn Schutzfaktoren gemeinsam auftreten, verstärkt sich ihr protektiver Effekt (Newcomb \& Felix-Ortiz, 1992).

Die personalen, kindbezogenen Ressourcen wie weibliches Geschlecht, erstgeborenes Kind, positives Temperament, positives Selbstwertgefühl, überdurchschnittliche Intelligenz und positives Sozialverhalten (Laucht et al., 1997) stehen in einer deutlichen Beziehung zur kindlichen Resilienz (Widerstandsfähigkeit). Hiermit wird die Fähigkeit des Kindes beschrieben, mit eigenen Kompetenzen schwierige und belastende Phasen in der Entwicklung erfolgreich zu bewältigen und zu verarbeiten (Egeland et al., 1993; Laucht et al., 1997). 
Allerdings erweist sich die Resilienz als nicht von Geburt an vorhandenes Merkmal, sondern als eine durch Erfahrungen und Lernprozesse erst im Laufe der Entwicklung entstandene Eigenschaft (Egeland et al., 1993).

Anhand des Rochester Child Resilience Project konnte aufgrund verschiedener kindbezogener Ressourcen eine Vorhersage getroffen werden, ob Kinder, die unter schwierigen Lebensumständen aufwachsen oder denen kritische Lebensereignisse zuteil werden, später eine fehlangepasste Entwicklung zeigen oder aber eine deutliche Resilienz aufweisen. Diese Ressourcen waren die Empathie, ein realistischer Attributierungsstil, soziale Problemlösungsfertigkeiten und ein hohes Selbstwertgefühl (Cowen et al., 1997).

Soziale Ressourcen beinhalten Faktoren, die umgebungsbezogen auf das Kind einwirken. Unterteilt wird hierbei in Schutzfaktoren innerhalb der Familie und in Schutzfaktoren innerhalb des sozialen Umfeldes. Innerhalb der Familie spielt die MutterKind-Interaktion eine wesentliche Rolle für die Entwicklung resilienter Merkmale des Kindes. Weiter stellen ein offenes, unterstützendes Familienklima, ein familiärer Zusammenhalt und Modelle positiver Bewältigung soziale Ressourcen dar (Laucht et al., 1997).

\subsubsection{Zusammenwirken von Risiko- und Schutzfaktoren}

Das Zusammenwirken von Risiko- und Schutzfaktoren entscheidet letzten Endes darüber, ob das Kind in der Lage ist, erschwerte Lebenssituationen erfolgreich zu kompensieren (Laucht et al., 1997). Die Schwierigkeit des Zusammenwirkens von Risiko- und Schutzfaktoren ist im großen Überschneidungsbereich der beiden Faktoren zu sehen. So zeigen protektive Faktoren ihr prädiktives Gewicht häufig nur dadurch, dass sie die mangelnde Präsenz von Risikofaktoren anzeigen. Risikofaktoren werden in der Kinder- und Jugendpsychiatrie klar definiert und besitzen zuverlässige Messverfahren. Schutzfaktoren hingegen können häufig nur unzureichend definiert und erfasst werden (Laucht et al., 1998). Vorsicht sollte allerdings dabei geboten sein, das Fehlen von Risikofaktoren als risikomildernd bzw. protektiv darzustellen. Die schützende Potenz der risikomildernden Faktoren kommt nur dann zum Tragen, wenn sich in der kindlichen Entwicklung ein ungünstiger Verlauf zeigt. Hierfür spricht auch die These, dass sich die Schutzfaktoren bei Kindern mit normaler, unbeein- 
trächtigter Entwicklung eher unbedeutend auf das Auftreten von psychischen Störungen auswirken (Stattin und Magnusson, 1996).

Somit zeigt sich die Potenz der Schutzfaktoren nur beim Vorliegen von risikoerhöhenden Faktoren (Rutter 1990).

Zusammenfassend ist nun deutlich geworden, dass der Entwicklungsverlauf eines Kindes und dessen Grad der Belastung nur dann beurteilbar ist, wenn eine Gegenüberstellung seiner Risikofaktoren im kind- und umgebungsbezogenen Bereich, seine Schutzfaktoren und auch seiner Resilienz- und Vulnerabilitätsfaktoren als Ganzes erfolgt (Scheithauer \& Petermann, 1999).

Regulationsstörungen gehören wie dargestellt zu den Risikofaktoren. Sie können als sekundäre Vulnerabilitätsfaktoren betrachtet werden, also als Merkmale des Kindes, welche sich erst in der Auseinandersetzung mit der sozialen Umwelt entwickeln. Hierbei, so zeigt der Literaturüberblick, wird vielfach angenommen, dass „negative Emotionalität“ oder „ein schwieriges Temperament des Säuglings“ am Beginn der „ungünstigen Entwicklung“ gestanden haben könnten. Das heißt, dass das Temperament (endogener Risikofaktor) und die Erfahrungen des Kindes in seiner sozialen Umwelt (Mutter-Kind-Beziehung, soziale Ressource) im Verlauf des ersten Lebensjahres zur Ausbildung des sekundären Vulnerabilitätsfaktors „Regulationsstörung“ geführt haben könnte. Die Anzahl empirischer Studien zu dieser These ist jedoch gering. Ziel der vorliegenden Dissertation ist daher deren Überprüfung.

Zunächst erfolgt jedoch eine vertiefende Darstellung der elterlichen Merkmale, die an der Entwicklung von Regulationsstörungen beteiligt sein könnten. 


\subsection{Elterliche Merkmale (Depressivität, Ängstlichkeit, psy- chisch/soziale Risiken, sozio-ökonomischer Status)}

\subsubsection{Mutter-Kind-Interaktion}

Eine wichtige Aufgabe der Eltern bzw. der Mutter besteht im ersten Lebensjahr in der unterstützenden Funktion der kindlichen Erregungs- und Emotionsregulation. Die Mutter-Kind Beziehung basiert auf drei Funktionen. Die Nähe der Mutter vermittelt dem Kind Sicherheit in ungewohnten Situationen. Sie fördert das Kind im Erkunden seiner Umwelt und im Lernprozess. Das Kind erwirbt zudem Bewältigungsstrategien für verschiedene Anpassungsaufgaben. Die Aufgabe der Eltern besteht hierbei nicht nur darin, die körperlichen und geistigen Grundbedürfnisse wie Pflege, Ernährung, Schutz und emotionale Sicherheit zu gewährleisten, sondern auch in der Unterstützung des Kindes bei der Entdeckung seiner Umwelt. Hierfür besitzen Eltern intuitive Voraussetzungen (Sarimski \& Papousek, 2000; Sroufe, 1996).

Das Ende des ersten Lebensjahres zeigt sich als ein bedeutsamer Zeitraum, in dem sich Entwicklungsveränderungen hinsichtlich der Selbstregulationsfähigkeiten des Kindes äußern (Rothbart \& Bates, 1998; Ruff \& Rothbart, 1996). Im ersten Lebensjahr ist die Unterstützung der Bezugsperson unbedingt erforderlich und entscheidend für die beginnende Selbstregulation des Kindes (Kopp, 1989; Sroufe, 1996; Thompson, 1994) Wichtig ist hier ein aktives, beteiligtes und akzeptierendes Verhalten in der Beziehung zum Kind, d.h. ein auf die kindlichen Signale zuverlässiges und adäquates Reagieren. In einer Studie von Bridges et al. (1997) an 64 Säuglingen im Alter von 12 bis 14 Monaten zeigte sich, dass ein aktives, zugewandtes elterliches Interaktionsverhalten mit positivem Emotionsausdruck und höherer Selbstregulationsfähigkeit des Kindes verknüpft und im Gegensatz dazu ein passiv zurückgezogenes Elternverhalten mit einer weiterhin ungünstigen Entwicklung des Kindes assoziiert war. 


\subsubsection{Mütterliche Depressivität und Ängstlichkeit}

Das Verhalten des Kindes gerade im ersten Lebensjahr ähnelt spiegelbildlich den schon beschriebenen physischen und emotionalen Fähigkeiten seiner nächsten Bezugsperson (Sroufe, 1996; Tronick, 1989). In den meisten Fällen stellt die Mutter diese Bezugsperson dar und prägt das Kind infolgedessen durch ihre Charaktereigenschaften, ihre Stimmungslage sowie ihr Verhalten in umfangreichem Maße.

Folglich stellt eine intakte Mutter-Kind-Interaktion eine Unterstützung für die personalen Ressourcen des Kindes dar (Laucht et al., 1998).

Field (1994) und Tronick (1989) beobachten bei depressiven Müttern eher die Tendenz zur stärkeren Passivität, weniger Einfühlungsvermögen und einem vermehrt negativen Emotionsausdruck im Gegensatz zu nichtdepressiven Müttern. Kinder von depressiven Mütter zeigen eher negative Emotionen in der Mutter-Kind-Interaktion. Folglich scheinen die Mütter durch Angst und Belastung in ihrer elterlichen Rolle gar nicht in der Lage zu sein, eine situationsangepasste Kommunikation im Umgang mit ihrem Kind zu zeigen (Mayseless, 1998).

So stellt sich die frühkindliche Erfahrung mit Emotionen und deren Verarbeitung als grundlegende Struktur der Entwicklung ihrer späteren persönlichen affektiven Möglichkeiten dar. Infolgedessen zeigen besonders depressive Mütter durch ihre reduzierten positiven Interaktionen eine Beeinflussung der emotionalen Entwicklung des Kindes (Kogan \& Carter, 1996). Interessanterweise scheint die Depressivität der Mutter nur im frühen Kindesalter nachhaltig zu wirken. In Studien, welche bei älteren Kleinkindern und Vorschulkindern durchgeführt wurden, kam man zu dem Ergebnis, dass die Depression der Mutter als alleiniges Merkmal nicht ausreicht, um vorhersagend das Verhalten des Kindes zu beeinflussen (Murray \& Cooper, 1997).

\subsubsection{Soziale und Sozio-ökonomische Merkmale}

Das heranwachsende Kind wird ebenfalls mit sozio-ökonomischen Einflüssen aus seiner Umwelt konfrontiert. Diese setzen sich unter anderem aus dem Lebensumfeld, der finanziellen Lage der Eltern und deren Bildungsniveau zusammen. Im Hinblick auf die Auswirkungen der genannten Einflüsse finden sich in der Literatur unterschiedliche Auffassungen. 
So beschreiben unterschiedliche Studien sozioökonomische Mangelbedingungen als Stressoren bezüglich kindlicher und elterlicher Entwicklung. Dementsprechend fanden sich bei Kindern aus schlechten sozio-ökonomischen Verhältnissen vermehrt Verhaltensprobleme (Campbell et al., 1986), unsicherere Mutter-Kind Beziehungen (Spieker \& Booth, 1988) und im fortgeschrittenem Lebensalter verminderte Sprachund Lesefähigkeiten sowie geringere schulische Leistungen (Walker et al., 1994). McLoyd und Wilson (1991) stellten eine erhöhte Stressanfälligkeit sowie vermehrte Angst- und Depressionsneigungen bei wirtschaftlich schlecht gestellten Eltern im Vergleich zu finanziell gesicherten Verhältnissen fest. Dem Kind wird in diesem Fall lediglich eine suboptimale Erziehung dargeboten. Darüber hinaus zeigten Werner und Smith (1992) in ihrer Arbeit auf, dass das Maß an positiven Eltern-Kind Interaktionen bei einem höheren Bildungsniveau der Eltern umfangreicher ist.

In einer weiteren Untersuchung beschreibt Fish (1999) die vermehrt negativen Emotionen und Zukunftsaussichten bei Müttern mit niedrigem sozialem Status im Vergleich zu Müttern mit hohem Sozialstatus. 


\section{Fragestellung}

Die vorliegende Arbeit soll einen Beitrag zum besseren Verständnis der Entwicklung des Schlafverhaltens von Säuglingen im Alter bis zu 12 Monaten leisten. Die Hauptaufgabe besteht in der Untersuchung möglicher Prädiktoren der Schlafverhaltensentwicklung der Säuglinge im Verlauf des ersten Lebensjahres. Hierzu werden Verhaltensbeobachtungen und kontrollierte Verhaltenstests eingesetzt.

Es sollen Zusammenhänge zwischen dem Schlafverhalten des Kindes in Form der Aufwachhäufigkeit pro Nacht sowie der Anzahl der Nächte mit Aufwachepisoden pro Woche im Alter von 12 Monaten mit den zeitlich vorausgegangenen Eigenschaften negatives/ positives Temperament (negative Emotionalität, positive Emotionalität) sowie Depressivitäts- und Angstneigung der Mutter untersucht werden. Weitere potentielle Einflussfaktoren wie Körpergewicht, Stilldauer, kognitive Reife, Koliken und Arztkonsultationen werden kontrolliert.

In der Literatur sind aussagekräftige Untersuchungen zur Entwicklung des Risikofaktors „Regulationsstörung“ im ersten Lebensjahr bei Säuglingen rar: Die Mehrheit der Studien zum Thema "Regulationsstörung" betrachtet das Mutter-Kind-System und fokussiert dabei das Konzept der Beziehungsstörung (Emde, 1987). Hierbei zeigen sich zwar auch mögliche Verhaltensmerkmale, jedoch nur in der Mutter-KindVerbindung und nicht für den Säugling als einzelnes Individuum. Insofern besteht die Gefahr der nicht eindeutigen Zuordnung von Störungen des Säuglings und Störungen der Mutter.

Hält man sich vor Augen, dass nach Wolkes et al. (2002) Meinung die Prävalenz von Verhaltens- und Schlafproblemen sowie kindliche Temperamentseigenschaften im Alter von 6 Monaten nicht nur temporäre, d.h. zeitlich begrenzte Probleme mit sich bringen, sondern noch im fortgeschrittenen Kindesalter durch ein erhöhtes Risiko an Hyperaktivität und verminderten schulischen Leistungen Bestand haben, so verlangt dies im Angesicht der doch weitreichenden Folgen von Regulations- und Schlafproblemen ein vermehrtes Interesse und Handlungsbereitschaft sowie eine differenzierte Betrachtung und Unterscheidung der Merkmale der Mutter und der Merkmale des Kindes.

Deshalb soll in dieser Arbeit, im Vergleich zu anderen durchgeführten Studien, zunächst die Beobachtung des Säugling als eigenständiges Individuum im Hinblick auf 
seine positiven/negativen Temperamentscharakteristika, sein Verhalten sowie seine körperliche und geistige Entwicklung in möglicher Korrelation mit seinem Schlafverhalten im Vordergrund stehen. Erst im weiteren Verlauf erfolgt die Einbeziehung der mütterlichen Merkmale sowie deren Verhaltensmuster im Hinblick auf die Schlafeigenschaften ihres Kindes.

Das Hauptaugenmerk der vorliegenden Arbeit soll auf folgende Fragen gerichtet sein:

- Welche Zusammenhänge gibt es zwischen den zeitlich vorausgehenden Temperamentsmerkmalen eines Säuglings und dessen Schlafverhalten (Aufwachepisoden pro Nacht, Anzahl der Nächte mit Aufwachepisoden pro Woche)? Das heißt: Lässt sich die Entwicklung des Schlafverhaltens aus den Temperamentsmerkmalen (positive und negative Emotionalität) vorhersagen?

- Ist ein Zusammenhang von unsicherem und depressivem mütterlichen Verhalten mit dem kindlichen Schlafverhalten aufzeigbar? Das heißt: Lässt sich die Entwicklung des Schlafverhaltens aus den mütterlichen Merkmalen (Depressivitäts- und Angstneigung) vorhersagen?

Darüber hinaus werden nachfolgende Zusammenhänge mit untergeordneter Priorität kontrolliert:

- Besteht ein Zusammenhang zwischen der kognitiven Reife und dem Schlafverhalten des Säuglings (Aufwachepisoden pro Nacht, Anzahl der Nächte mit Aufwachepisoden pro Woche)?

- Ist die Entwicklung des kindlichen Schlafverhaltens aus dem Auftreten von „Koliken“ und der Anzahl der Arztkonsultationen vorhersagbar?

- Welche Bedeutung hat das Stillverhalten und die Stilldauer der Mutter und der damit verbundene Ernährungszustand des Kindes für die Entwicklung des Schlafverhaltens? 


\section{Methodenteil}

\subsection{Stichprobe und Untersuchungsgang}

\subsubsection{Zusammensetzung der Stichprobe}

Der Gesamtumfang der Stichprobe setzt sich aus insgesamt 101 BezugspersonenKind-Paaren zusammen. Diese Studie erhält seit 1997 eine Förderung durch die Deutsche Forschungsgemeinschaft (DFG) (Kennziffer: Pa 543/2-1, 2-2, 2-3, 2-4) (Pauli-Pott, 2001).

Die folgenden Ausführungen wurden auf der Basis von Pauli-Pott (2001) zusammengefasst:

Die Drop-Out Rate der Gesamtgruppe der 101 Familien bis 12 Monate kann mit $5,9 \%$ angegeben werden, was im Literaturvergleich als gering zu beurteilen ist.

\subsubsection{Stichprobenkriterien}

Um die Anzahl potentiell moderierender Variablen zu reduzieren, wurde die Stichprobe insofern homogenisiert, als dass ausschließlich Erstgebärende mit gesundem Säugling in die Stichprobe aufgenommen wurden. Des Weiteren bestand als Voraussetzung das Zusammenleben der Eltern sowie gute Deutschkenntnisse. Ausschlusskriterien wurden wie folgt definiert: Schwerwiegende Geburtskomplikationen, Geburtsgewicht < 2500g, Vorliegen einer Asphyxie, Vorliegen von Fehlbildungen und chronischen Erkrankungen des Kindes sowie Mehrlingsgeburten.

\subsubsection{Stichprobenzusammenstellung}

Die Zusammenstellung der Stichproben erfolgte in den Entbindungsstationen zweier Gießener Krankenhäuser. Die Rekrutierung der Stichprobe fand zwischen Juli 1997 
und März 1998 statt. Im Verlauf dieses Dreivierteljahres kam es zu insgesamt 85 Besuchen der oben genannten Einrichtungen.

Sowohl das katholische St. Josefs Krankenhaus als auch das Evangelische Krankenhaus dienen der allgemeinen Krankenversorgung in Gießen und sind auf dem Gebiet der Geburtshilfe bezüglich ihrer Geburtenzahlen und ihrer Angebote für die schwangeren Frauen vergleichbar.

In beiden Einrichtungen bestand eine Kooperation mit den Belegärzten der Entbindungsstation (Prof. Dr. Schubring; Drs. Esch, Felck und Meyer). Bei den „Rekrutierungsbesuchen“ wurden mittels Befragung der Säuglingsschwestern die Namen aller Frauen zusammengestellt, welche in den letzten Tagen entbunden hatten und die Aufnahmekriterien in die Stichprobe erfüllten (Erstgebärende mit gesundem Kind). Diese Frauen erhielten nähere Informationen bezüglich der Studie. Bei Einwilligung zur Studienbeteiligung erhielten die Mütter ein Informationsblatt; ein Termin für einen telefonischen Kontakt wurde abgesprochen. Dieses telefonische Gespräch erfolgte dreieinhalb Monate später, circa 14 Tage vor dem ersten Untersuchungstermin.

Die Gesamtzahl der bei den Besuchen in Frage kommenden Mütter, welche an der Studie teilnahmen, betrug $61,5 \%$.

\subsubsection{Stichprobenbeschreibung}

Die untersuchte Gesamtstichprobe kann wie folgt beschrieben werden:

Entsprechend den Kriterien, welche für diese Stichprobe festgelegt wurden, handelte es sich bei den 101 Säuglingen ausschließlich um erstgeborene, gesunde Kinder. 42,6 \% (43) hiervon waren Mädchen, 57,4 \% (58) Jungen. In drei Familien fand im Verlauf des ersten Lebensjahres ein Wechsel der primären Bezugsperson statt. Die Eltern nahmen abwechselnd Erziehungsurlaub. Aufgrund dieses Wechsels wurden die drei Familien bei den längsschnittlichen statistischen Analysen nicht berücksichtigt.

Der Geburtsverlauf stellte sich bei mehr als der Hälfte der Frauen spontan und ohne nennenswerte Komplikationen dar. Bei 29,7 \% war jedoch ein Kaiserschnitt notwendig. Die Apgar-Werte nach 5 und 10 Minuten lagen dementsprechend alle im oberen Wertebereich (> 7). Die Neugeborenen hatten ein durchschnittliches Geburtsgewicht 
zwischen $2500 \mathrm{~g}$ und $4650 \mathrm{~g}(\mathrm{x}=3414,64 \mathrm{~s}=441,15)$. Bei der Vorsorgeuntersuchung U3 (4-6 Wochen) schwankte das Körpergewicht zwischen $3280 \mathrm{~g}$ und $6420 \mathrm{~g}$ (x $=4474 \mathrm{~s}=616,91)$ und die Körpergröße zwischen 50 und $63 \mathrm{~cm}(x=55,5 \mathrm{~s}=2,2)$.

Hinsichtlich ihrer körperlichen und geistigen Entwicklung zeigte der Großteil der Kinder in den Vorsorgeuntersuchungen U1 bis U3 keinerlei Auffälligkeiten. Nur bei 22 Säuglingen bestanden bei wenigstens einer der Vorsorgeuntersuchungen kleinere Probleme wie Neugeborenenexanthem, leichte Sichelfußstellung, Hüftgelenksdysplasie und Nabelgranulome. Ärztliche Hilfe in der Praxis bzw. in einem Krankenhaus wurde in den ersten vier Monaten zwischen null- und neunmal von den Eltern der Säuglinge in Anspruch genommen. Hierbei ist anzumerken, dass 91,8\% (90) der Säuglinge, ausgenommen die Vorsorgetermine, maximal dreimal einem Arzt vorgestellt wurden. 59,4 \% (60) wurden im Alter von vier Monaten voll gestillt.

Der Altersdurchschnitt der Mütter lag im Schnitt bei 28,8 Jahren ( $s=3,7$ ), bei den Vätern bei circa 31,6 Jahren ( $s=4,8)$. Das Bildungsniveau betreffend wiesen 19,9\% der Eltern einen Hoch- oder Fachhochschulabschluss auf. Beim Schulabschluss überwog das Abitur mit 40,8 \% der Mütter und 41,8 \% der Väter. Real- und Hauptschulabschlüsse waren sowohl bei den Müttern mit 32,7\% (Realschulabschluss) bzw. 7,1 \% (Hauptschulabschluss), als auch bei den Vätern mit 29,6 \% (Realschulabschluss) bzw. 8,2 \% (Hauptschulabschluss) im Gesamtvergleich geringer vertreten. 83 (82,8 \%) Väter gingen zum Zeitpunkt der Erstuntersuchung einer beruflichen Vollzeitbeschäftigung nach, 12 Väter (11,9\%) befanden sich in einer Ausbildung und 6 Väter (5,9 \%) waren nicht berufstätig. Hiervon waren vier ohne Arbeit, ein Vater war Frührentner und ein Vater im Erziehungsurlaub.

Dahingegen waren 81 Mütter (80,2 \%) nicht berufstätig, 9 (8,9 \%) gingen einem Studium nach und weitere $9(8,9 \%)$ gaben an, stundenweise einer Tätigkeit bzw. einer Halbtagesbeschäftigung nachzugehen. Lediglich zwei Mütter (2,0 \%) waren voll berufstätig. 


\subsubsection{Ablauf der Untersuchungen}

\subsubsection{Zeitpunkte und Zeiträume der Untersuchungen}

Insgesamt wurden drei Erhebungen im ersten Lebensjahr durchgeführt, die erste im Alter von 4 Monaten, die nachfolgenden im 8. und 12. Lebensmonat. Pro Erhebung wurden drei Untersuchungstermine im Abstand von 7 Tagen vor bis 7 Tage nach dem Tag, an dem das Baby genau 4, 8 oder 12 Monate alt wurde, durchgeführt.

\subsubsection{Untersuchungsgang}

Es wurden pro Mutter-Kind-Paar und Erhebungszeitpunkt zumeist drei Untersuchungstermine durchgeführt. Die Termine wurden zu unterschiedlich festgelegten Tageszeiten (siehe unten) an drei Tagen innerhalb einer Woche vereinbart. Diese unterteilten sich in einen Termin im Videoraum und in zwei weitere Hausbesuche. Zum Abschluss des Videotermins wurde der Mutter ein Fragebogen ausgehändigt, den sie bis zum Termin der häuslichen Beobachtung komplett bearbeitet haben sollte.

\section{Der Videotermin am Vormittag}

Der Termin wurde nach Absprache mit der Mutter für den Verlauf des Vormittags festgelegt und zwar für die Zeit in der das Kind nach Ansicht der Mutter gesättigt und wach sein müsste. Zu Beginn wurde ein strukturiertes Interview mit der Mutter durchgeführt. Unter Zuhilfenahme des Kinderuntersuchungsheftes wurde die Mutter zum Gesundheitszustand des Kindes befragt. Der Fragenkatalog enthielt Fragen zum Geburts-/Schwangerschaftsverlauf, zu Auffälligkeiten oder Erkrankungen während der Vorsorgeuntersuchungen, zu derzeitigen Erkrankungen (Erkältung, Fieber), zum Ernährungsverhalten sowie als wichtigen Punkt Fragen zum Schlafverhalten des Kindes. Im Alter von 4 Monaten wurden u.a. kindliche „Koliken“ erfragt. (siehe Anhang C) 
Im Anschluss daran wurde der „Bayley Mental Test“ (Bayley, 1993) durchgeführt. Die Dauer der Bayley-Untersuchung betrug im Alter von 4 Monaten ungefähr 15 Minuten, im Alter von 8 Monaten etwa 25 Minuten und im Alter von 12 Monaten etwa 30 Minuten. Dem Bayley-Test folgte eine zehnminütige „Wickel-Spiel-Sequenz“ (nach Esser et al., 1993) zwischen Mutter und Säugling.

Ein Abbruch des Entwicklungstestes erfolgte lediglich dann, wenn es der Verhaltenszustand des Kindes erforderte. Der Test wurde dann allerdings bei einem weiteren Hausbesuch komplettiert.

Zu einem späteren Zeitpunkt wurden zwei weitere Hausbesuche durchgeführt, deren Ergebnisse jedoch im Rahmen dieser Arbeit nicht berücksichtigt werden und daher auf eine nähere Erläuterung verzichtet wird. 


\subsection{Erfassungsmethoden}

Im folgenden Kapitel werden die Erfassungsmethoden jener Variablen näher erläutert, welche zur Bearbeitung und Beantwortung der Fragestellung der hier vorliegenden Arbeit benötigt wurden. Eine zusammenfassende Übersicht dieser Parameter stellt Tabelle 1 dar.

Tabelle 1: Übersicht der im Alter von $\underline{4,8}$ und 12 Monaten bei den Säuglingen durchgeführten Erhebungen

\begin{tabular}{|c|c|}
\hline Merkmal & Erfassungsmodus \\
\hline \multicolumn{2}{|l|}{ Hauptmerkmale: } \\
\hline Schlafverhalten des Säuglings & $\begin{array}{l}\text { - Strukturiertes Interview mit der } \\
\text { Bezugsperson }\end{array}$ \\
\hline $\begin{array}{ll}\text { Temperament: } \\
\text { - } & \text { Negative Emotionalität/ Irritierbarkeit } \\
\text { - } & \text { Positive Emotionalität }\end{array}$ & $\begin{array}{ll}\text { - } & \text { Elternfragebogen } \\
\text { - } & \text { Reaktionen auf standardisierte Reize } \\
& (\text { „Verhaltenstest“) }\end{array}$ \\
\hline $\begin{array}{l}\text { Depressivität/ Ängstlichkeit der Bezugsper- } \\
\text { son }\end{array}$ & $\begin{array}{l}\text { Fragebogenskalen: } \\
\text { - „Depressivität“ und „Überfürsorge aus } \\
\text { Angst“ aus dem EMKK von Engfer } \\
\text { - „Trait-Angst“ aus dem STAI von Laux } \\
\text { et al. } \\
\text { - „Hoffnungslosigkeitsskala“ von Kram- } \\
\text { pen }\end{array}$ \\
\hline \multicolumn{2}{|l|}{ Kontrollmerkmale: } \\
\hline Kognitiver Entwicklungsstand & - Bayley-Mental Test \\
\hline $\begin{array}{l}\text { - } \text { „Koliken“ } \\
\text { - Stilldauer und Stillverhalten } \\
\text { - } \quad \text { Körpergewicht } \\
\text { - } \quad \text { Anzahl der Arztbesuche }\end{array}$ & $\begin{array}{l}\text { - Strukturiertes Interview mit der } \\
\text { Bezugsperson }\end{array}$ \\
\hline
\end{tabular}




\section{Erfassung der Hauptmerkmale}

\subsubsection{Erfassung des Schlafverhaltens der Säuglinge}

Das kindliche Schlafverhalten wurde anhand von strukturierten Interviews mit der Bezugsperson zum Zeitpunkt des achten und zwölften Lebensmonates erfasst. Im Alter von 8 Monaten bezogen sich die Fragen auf die Dauer des nächtlichen Schlafes ohne Unterbrechung (18:00 bis 6:00 Uhr Schlafbeginn), die Anzahl der Schlafperioden am Tag sowie die Regelmäßigkeit des Tagschlafes. Im Alter von 12 Monaten bezogen sich die Fragen auf die nächtliche Durchschlaffähigkeit (18:00 bis 6:00 Uhr) des Kindes, die nächtliche Schlafdauer ohne Unterbrechung (18:00 bis 6:00 Uhr Schlafbeginn), die Anzahl der Nächte mit Aufwachepisoden pro Woche sowie die Aufwachepisoden pro Nacht. Weiter wurde zu diesem Zeitpunkt erfragt, ob das Kind bei Aufwachepisoden länger als 20 Minuten wach ist und es anschließend zum Einschlafen länger als 30 Minuten benötigt, ob das Kind zeitweise auch im Elternbett schläft, wie hoch die Anzahl der Schlafperioden am Tag ist und deren Regelmäßigkeit. Wolke (1999) spricht von Durchschlafproblemen, wenn das Kind älter als 6 Monate ist und an 5 Nächten pro Woche mindestens einmal pro Nacht (zwischen 0:00 und 5:00 Uhr) aufwacht und von schweren Durchschlafproblemen, wenn das Kind mehrmals pro Nacht aufwacht (Wolke, 1999). Die Deutsche Gesellschaft für Kinderund Jugendpsychiatrie definiert Durchschlafstörungen als durchschnittlich mehr als dreimal nächtliches Aufwachen in mindestens 4 Nächten der Woche verbunden mit der Unfähigkeit, ohne elterliche Hilfen alleine wieder einzuschlafen (Deutsche Gesellschaft für Kinder- und Jugendpsychiatrie, 2003).

In diesen Definitionen sind die Anzahl der Nächte mit Aufwachepisoden pro Woche und die Anzahl der Aufwachepisoden pro Nacht vermischt. Hier sollen die zwei in den Definitionen angesprochenen Aspekte als zwei unabhängige Variablen verwendet werden. Daher werden folgende Zielkriterien benutzt:

a) Anzahl der Nächte mit Aufwachepisoden pro Woche

b) Anzahl der Aufwachepisoden pro Nacht im Alter von 12 Monaten. 


\subsubsection{Erfassung der Temperamentsmerkmale}

Entsprechend den Fragestellungen werden die Temperamentsmerkmale negative Emotionalität/ Irritierbarkeit und positive Emotionalität zu den drei Erhebungszeitpunkten im ersten Lebensjahr im Folgenden aufgeführt. Eine Aufstellung operationaler Definitionen dieser Dimensionen verfasste Rothbart (1981) im Rahmen der Konstruktion des „Infant Behavior Questionnaire“. Diese dienten als Grundlage der vorliegenden Untersuchungen:

Negative Emotionalität/ Irritierbarkeit betrifft negatives emotionales Ausdrucksverhalten (Mimik, Vokalisation) des Säuglings in von Frustration geprägten Situationen.

Gegensätzlich hierzu wird das Ausmaß des positiven emotionalen Ausdrucksverhaltens (Mimik, Vokalisation) als positive Emotion dargestellt.

Um die Möglichkeit der wechselseitigen Validierung zu erreichen, wurden jeweils mehrere Methoden eingesetzt:

Zur Erfassung der oben genannten Temperamentsdimensionen wurden

- schriftliche Befragungen der Hauptbezugspersonen und

- Beobachtungen von Reaktionen auf standardisierte Reize (Verhaltenstests) eingesetzt.

\subsubsection{Erfassung der Temperamentsmerkmale im Elternurteil}

Die Eltern bearbeiteten die deutschsprachige Adaptation des "Infant Behavior Questionnaire“ (IBQ) von Pauli-Pott et al. (2003) im Alter von 4, 8 und 12 Monaten. Der Fragebogen ist reliabel und valide.

Die Reliabilitätsschätzungen der Skala „Unbehagen bei Einschränkung“ betragen .79 (interne Konsistenz) und .87 (Testhalbierung) im Altersbereich 3 bis 4 Monate, .82 (interne Konsistenz) und .90 (Testhalbierung) im Altersbereich 6 bis 8 Monate und .81 (interne Konsistenz) und .84 (Testhalbierung) im Altersbereich 10 bis 12 Monate. Die internen Konsistenzkoeffizienten der Skala "Lächeln und Lachen“ liegen bei .79, .80 und .77, die Testhalbierungskoeffizienten bei .86, .82, und .84 in den genannten Altersbereichen.

Zur Validität vergleiche Abschnitt 4.2.2.3. 


\subsubsection{Erfassung der Temperamentsmerkmale durch Untersuchungen von Reaktionen auf standardisierte Reize (Verhaltenstest)}

Die Verhaltensbeobachtung in standardisierten Testsituationen ist als die intern valideste Methode zur Erfassung frühkindlicher Temperamentsmerkmale zu betrachten (Pauli-Pott et al., 2005). Folgende Verfahren wurden im Rahmen der Gießener Längsschnittsstudie zur Temperamentsentwicklung entwickelt und validiert (vgl. Pauli-Pott et al., 2004): Eine Untersuchungsleiterin nahm die Applikation der Reize vor. Die Aufzeichnung der Reaktionen des Säuglings auf diese Reize erfolgte videographisch. Um eine möglichst aussagekräftige, objektive Beurteilung zu erhalten, wurde die Beurteilung der gezeigten Reaktionen nochmals durch das Urteil einer weiteren Mitarbeiterin, welche nicht an der Untersuchungsdurchführung beteiligt war, gesichert. Diese Mitarbeiterin stand nun nochmals unter Kontrolle einer weiteren Person, welche wiederum eine Beurteilung abgab. Hierdurch sollte eine möglichst hohe Kontrolle der systematischen Verzerrung erreicht werden. Es kam somit zu einem Ausschluss denkbarer Konfundierungen mit mütterlichen Merkmalen.

In dieser Studie wurden die Merkmale „positive Emotionalität“ und „negative Emotionalität/Irritierbarkeit" anhand dieser Methode erfasst. Zur sprachlichen Vereinfachung der folgenden Darstellung soll der Terminus „Verhaltenstest“ zur Bezeichnung dieser Methode verwendet werden.

\subsection{Erfassung negativer Emotionalität/ Irritierbarkeit im Alter von 4 Monaten}

Im Alter von 4 Monaten wurden standardisierte Reize von mittlerer bis hoher Intensität dargeboten. Geeignet hierfür erschienen die unten genannten Items aus dem „Bayley Mental Test“ (Bayley, 1993).

Die Bayley-Skalen beinhalten unter anderem ein Ratingverfahren (das „Infant Behavior Record“, IBR) zur Beurteilung verschiedener Verhaltensmerkmale der Säuglinge. Eine Interpretation der Skalen des IBR als „Temperamentsmerkmale“ kam häufig vor (Matheny et al., 1974; Roth et al., 1984). Infolge ihrer Altersplatzierung für die Untersuchung viermonatiger Säuglinge wurden daher die Items der "Bayley-Mental-Scale“ mit standardisierten Vorgaben und konstanter Reihenfolge verwendet. Um möglichst 
genaue Ergebnisse der Untersuchung zu erhalten, wurde zur Vermeidung von Ermüdung und Überanstrengung des Säuglings die Gesamtdauer der Prozedur auf 10 Minuten begrenzt. Trotz dieser zeitlichen Beschränkung war es in insgesamt 10 Fällen nicht möglich, den Untersuchungsverlauf während des Termins im Videolabor vollständig durchzuführen, da die Sequenz durch Einschlafen oder Schreien des Säuglings unterbrochen wurde.

Die folgenden Items und Itemsequenzen wurden zur Ermittlung der negativen Emotionalität/ Irritierbarkeit verwendet. Sie wurden in der folgenden Reihenfolge durchgeführt.

1. Roter Ring, Baby in Rückenlage (Items 37, 46, 33, 44)

Das Baby liegt auf einem Wickeltisch. Ein roter Ring an einer Schnur wird in greifbarer Nähe über dem Säugling bewegt. Der Säugling erhält den Ring für eine Zeit. Dann wird der Ring durch die Untersucherin entfernt.

2. Glocke/ Rassel (Item 28)

Das Baby liegt auf dem Wickeltisch. Abwechselnd wird auf der linken und rechten Seite zunächst eine Glocke geläutet und dann mit einer Rassel gerasselt.

3. Glocke und Rassel im Wechsel (Items 26, 59)

Das Baby liegt auf dem Wickeltisch. Abwechselnd werden die Glocke und die Rassel betätigt.

4. Rassel in der Hand (Items 36, 59)

Das Baby erhält dann die Rassel. Die Rassel wird wiederholt aus der Hand entfernt und dem Kind auf die Brust gelegt.

5. Glocke (Item 47)

Das Baby sitzt auf dem Schoß der Mutter mit Blick zur Mutter. Die Glocke wird außerhalb des Gesichtsfeldes links und rechts neben dem Kind geläutet.

6. Rassel (Item 48)

(entspr. Item 47)

7. Roter Ball (Item 38)

Das Baby sitzt auf dem Schoß der Mutter vor einem Tisch (Blick zur Untersucherin), Ein roter Ball wird über den Tisch gerollt.

8. Roter Ring (Item 40)

(Position wie bei Item 38) Ein roter Ring an einem Band pendelt in greifbarer Nähe vor dem Kind.

9. Löffel (Item 41)

(Position wie bei Item 38) Ein Teelöffel wird vor dem Kind bewegt.

10. Roter Würfel (Items $32,49,51,54,60$ )

Ein roter Würfel wird zunächst außerhalb der Reichweite des Säuglings auf den Tisch gelegt. Nach einiger Zeit wird der Würfel näher zum Kind geschoben. Der Würfel ist für das Kind schwer zu erreichen. 
Zur adäquaten Beurteilung der affektiven Reaktion des Säuglings fand eine Adaptation der Skala „Kind Emotion“ der MBS-MKI-S von Esser et al. (1989) statt. Hierbei erfolgte in jedem Intervall zwischen Beginn (Gegenstand im Blickfeld des Säuglings oder Ertönen des ersten Geräusches) und Ende (Gegenstand wird aus dem Blickfeld des Säuglings entfernt, letztes Geräusch verklingt) der Darbietung des Items bzw. der Itemsequenz eine Bewertung des Affektausdrucks des Kindes. Ein Score „negative Emotionalität/Irritierbarkeit" wurde durch Summieren über die Werte der 10 Sequenzen erstellt. Die Beurteilerübereinstimmung lag hier bei Kappa = 1.00 (60 Intervalle).

\subsection{Erfassung negativer Emotionalität im Alter von 8 und 12 Monaten}

Geeignete Items aus dem „Bayley Test“ fanden ebenfalls Verwendung bei der Erfassung der negativen Emotionalität der Säuglinge im Alter von 8 und 12 Monaten. Im Alter von 8 Monaten wurden folgende Items und Itemsequenzen angewendet:

1. Roter Würfel (Items 60, 64, 70, 77, 82)

Ein kleiner roter Würfel wird außerhalb der Reichweite des Kindes auf dem Tisch platziert. Nach einer Zeit wird dieser und weitere Würfel vor das Kind in Reichweite auf den Tisch gelegt.

2. Tasse und Würfel (Items 90, 100)

Die Untersucherin demonstriert das Fallen eines Würfels in die Tasse. Das Kind wird zur Imitation aufgefordert.

3. Puppe und Tasse (Items 63, 73, 88)

Eine kleine rote Puppe wird auf den Tisch vor das Baby gesetzt und dann mit einer Tasse verdeckt.

4. Löffel und Tasse (Item 92)

Die Untersucherin rührt mit einem Teelöffel in einer Tasse. Das Kind erhält dann beide Gegenstände.

5. Dose (Items 62, 75)

Die Untersucherin legt eine kleine Dose auf den Tisch und schiebt diese dann über die Tischkante, so dass die Dose herunter fällt.

6. Spiegel (Item 65, 76)

Dem Baby wird ein Spiegel vorgehalten. 
7. Guck-Guck-Spiel (Item 81)

Die Untersucherin spielt mit dem Baby „Guck-Guck“.

8. Roter Ring (Items 67, 71, 80, 105)

Ein roter Ring an einer Schnur wird vor dem Kind gependelt. Der Ring wird dann außer Reichweite auf den Tisch gelegt. Die Schnur befindet sich in Reichweite.

9. Papier und Kreide (Items 74, 95, 98)

Die Untersucherin demonstriert das Malen mit der Kreide auf dem Papier. Das Kind erhält Kreide und Papier.

10. Glocke (Items 83, 78)

Dem Kind wird eine Glocke zum Spiel überlassen.

11. Rotes Auto und Kleenextuch (Item 86, 99)

Die Untersucherin zeigt dem Kind ein kleines rotes Auto. Das Kind erhält das kleine rote Auto. Nach einer Zeit nimmt die Untersucherin das Auto zurück und verdeckt es mit einem Kleenextuch.

12. Gelbes Brett mit Löchern (Item 87)

Vor das Baby wird auf dem Tisch ein gelbes Brett mit Löchern gelegt. Die Untersucherin steckt ihre Fingern in eines der Löcher.

13. Bilderbuch (Item 93)

Das Baby erhält ein Bilderbuch.

14. Blaue Dose (Item 91)

Das Baby beobachtet die Untersucherin. Sie legt zwei gelbe Perlen in eine blaue Dose und rasselt damit. Das Baby erhält dann die Dose ohne die Perlen.

15. Quietschpuppe (Item 104)

Die Untersucherin drückt auf den Bauch einer sehr harten Plastikpuppe, so dass diese quietscht. Dem Baby gelingt es nicht, die Puppe zum Quietschen zu bringen.

Im Alter von 12 Monaten fanden die folgenden Items und Itemsequenzen Gebrauch:

1. Spiegel (Item 76)

siehe 8 Monate: Punkt 6

2. Puppe und Kleenextuch (Item 86)

Eine kleine Puppe wird mit einem Kleenextuch verdeckt.

3. Roter Würfel (Items 82, 111)

Vor das Baby werden auf den Tisch drei Würfel gelegt. Später werden einige dazugelegt. Die Untersucherin baut einen Turm aus drei Würfeln und fordert das Kind auf, dies auch zu tun.

4. Tasse und Würfel (Items 88, 90, 100, 114)

siehe 8 Monate: Punkt 2

5. Löffel und Tasse (Item 92)

siehe 8 Monate: Punkt 4

6. Glocke (Item 83) 
siehe 8 Monate: Punkt 10

7. Blaue Dose (Item 91, 107)

Zunächst wird wie bei Punkt 14 (8 Monate) vorgegangen. Dann wird die Dose mit einem Deckel, in dem ein kleines Loch ist, verschlossen. Das Kind erhält Perlen mit der Aufforderung, diese in die Dose zu stecken.

8. Blaue Dose und kleiner Affe (Item 102)

In eine blaue Dose wird ein kleiner Affe gesteckt. Die Dose wird mit einem Deckel verschlossen. Das Baby erhält die verschlossene Dose.

9. Rote Dose (Item 115)

Die Untersucherin demonstriert wiederholt das Öffnen und Schließen einer kleinen runden roten Dose. Das Baby erhält die beiden Teile.

10. Rotes Auto (Item 99)

Nach Demonstration durch die Untersucherin fordert diese das Baby auf, ein kleines rotes Auto über den Tisch zu schieben.

11. Steckbrett mit gelben Stäben (Item 87, 108)

Vor das Baby wird ein Brett gelegt, in dem gelbe Stäbe stecken. Die Untersucherin entfernt dann die Stäbe und legt sie neben das Brett.

12. Bilderbuch (Items 93,103 ) siehe 8 Monate: Punkt 14

13. Papier und Kreide (Items 95, 98, 112) siehe 8 Monate: Punkt 9

14. Quietschpuppe (Item 104) siehe 8 Monate: Punkt 15

15. Roter Ring (Item 105) siehe 8 Monate: Punkt 8

16. Kleine Tablette und Dose (Item 109) Die Untersucherin steckt eine kleine Tablette in eine durchsichtige kleine hohe Dose. Das Baby erhält die Dose mit der Aufforderung, die Tablette herauszuholen. Dies gelingt nur, wenn die Dose umgedreht wird.

17. Blaues Brett mit Spielsteinen (Items 110, 121)

Das Baby erhält ein Brett mit verschieden geformten Vertiefungen und einen nur in einige der Vertiefungen passenden Spielstein. Falls es den Stein korrekt platziert, erhält es mehr Steine.

18. Kleiner Hase und Stock (Item 122)

Der kleine Hase liegt außer Reichweite auf dem Tisch und soll mit einem Stock herangeholt werden.

Die Hauptaufgabe dieser Items lag in der Konfrontation der Säuglinge mit attraktiven Gegenständen sowie im Wegnehmen dieser Gegenstände durch die Untersuchungs- 
leiterin. Des Weiteren wurden schwer lösbare, frustrierende Manipulationsaufgaben an die Säuglinge gestellt. Als Kennwerte der negativen Emotionalität (Ärgertendenz) wurde jeweils die Anzahl der Intervalle, in denen Ärgerreaktionen vorkamen, verwendet.

Eine Überprüfung der Beurteilerübereinstimmung wurde in 5 Fällen durchgeführt. Der Kappa-Wert lag für die 8-Monats-Untersuchung bei .94 (70 Episoden), für die 12Monats-Untersuchung bei .83 (68 Episoden).

\subsection{Erfassung von positiver Emotionalität/Soziabilität}

Die Bayley-Mental-Testuntersuchung setzt sich aus einer Interaktion zwischen einer freundlichen Untersuchungsleiterin und dem Säugling zusammen. Hierbei werden dem Säugling von der Untersuchungsleiterin attraktive Gegenstände angeboten, mit welchen dieser altersadäquate Aufgaben bewältigen soll. Somit waren auch die Items und Itemsequenzen des Bayley-Tests zur Erfassung des positiven Emotionsausdrucks geeignet. Die Durchführung der Bayley-Untersuchung erfolgte, wie oben schon beschrieben, in standardisierter Weise und in konstanter Abfolge der Items. Die gesamte Untersuchung wurde videographisch festgehalten.

Analog zur Erfassung der negativen Emotionalität wurde im Alter von 4, 8 und 12 Monaten die positive Emotionalität erfasst. Als Score „positive Emotionalität“ fand die Anzahl der Itemsequenzen, in denen positive Reaktionen beobachtet wurden, Verwendung.

Die Prüfung der Beurteilerübereinstimmung konnte auch hier als zufriedenstellend beurteilt werden. Der Kappa-Wert für die Untersuchung der 8monatigen Säuglinge lag bei .91 (70 Intervalle) bei den 12 monatigen Säuglingen bei .79 (67 Intervalle) (vgl. Pauli-Pott et al., 2004). 


\subsubsection{Interkorrelationen der Temperamentsmaße}

Zur Einschätzung der Validität der hier genutzten, neu entwickelten Maße war eine Bestimmung der Interkorrelationen der Maße innerhalb der zwei Merkmalsbereiche notwendig.

In Tabelle 2 werden die Interkorrelationen der positiven/negativen EmotionalitätsmaBe der Kinder im Alter von 4, 8 und 12 Monaten beschrieben.

Die Korrelationen zwischen Fragebogenskala und Verhaltenstest erweisen sich zu allen drei Zeitpunkten als statistisch bedeutsam.

Eine genaue Beschreibung der Interkorrelationen sowie der Darstellung der Zusammenhänge mit weiteren Verhaltensbeobachtungen des Kindes finden sich in PauliPott et al. (2004, 2005).

Insgesamt kann von einer zufriedenstellenden Validität der Verhaltenstests ausgegangen werden. 
Tabelle 2: Interkorrelation der positiven/ negativen Emotionalitätsmaße der Säuglinge im Alter von 4, 8 und 12 Monaten

\begin{tabular}{|c|c|c|}
\hline & \multicolumn{2}{|l|}{ Verhaltenstest } \\
\hline & $\begin{array}{l}\text { „positive Emotionali- } \\
\text { tät“ }\end{array}$ & $\begin{array}{l}\text { „"negative Emotio- } \\
\text { nalität“ }\end{array}$ \\
\hline \multicolumn{3}{|l|}{4 Monate } \\
\hline Fragebogenskala „Lächeln/ Lachen“ & $.33^{\star \star \star}$ & \\
\hline $\begin{array}{l}\text { Fragebogenskala „Unbehagen bei Ein- } \\
\text { schränkung“ }\end{array}$ & & $.24^{\star}(S p)$ \\
\hline \multicolumn{3}{|l|}{8 Monate } \\
\hline Fragebogenskala „Lächeln/ Lachen“ & $.26^{\star}$ & \\
\hline $\begin{array}{l}\text { Fragebogenskala „Unbehagen bei Ein- } \\
\text { schränkung“ }\end{array}$ & & $.20^{t}(\mathrm{Sp})$ \\
\hline \multicolumn{3}{|l|}{12 Monate } \\
\hline Fragebogenskala „Lächeln/ Lachen“ & $.24^{\star}$ & \\
\hline $\begin{array}{l}\text { Fragebogenskala „Unbehagen bei Ein- } \\
\text { schränkung“ }\end{array}$ & & $.23^{\star}(\mathrm{Sp})$ \\
\hline
\end{tabular}

Anmerkung: Ohne besondere Kennzeichnung Pearson-Produkt-Moment-Korrelationen;

Fallzahl zwischen 89 und 98.

Signifikanzniveau: $n s$ : nicht signifikant, t: $p<0,10,{ }^{*}: p<0,05,{ }^{* *}: 0,01,{ }^{* * *}: p<0,001$

\subsubsection{Erfassung der Depressionsneigung und Ängstlichkeit der Bezugsperson}

Die Befragung der Mutter bezüglich ihrer Selbstzweifel und Unzufriedenheit in der Mutterrolle sowie die Darstellung ihres Selbstbildes, ihrer persönlichen Erfahrungen und ihrer Zukunftserwartung diente zur Beurteilung der Depressionsneigung. Bei den Untersuchungen zu allen drei Erhebungszeitpunkten fanden die Skala „Depressivität“ und „Überfürsorge aus Angst“ aus dem „Fragebogen zur Erfassung der Erziehungseinstellungen von Müttern mit Kindern im Kleinstkindalter" (EMKK) von Engfer (1984) sowie die "Skala Hoffnungslosigkeit“ von Beck in der deutschen Bearbeitung von Krampen (1979) Anwendung. 
Die Skala „Trait-Angst" aus dem "State-Trait-Angstinventar“ (STAI) von Laux, Glanzmann, Schaffer und Spielberger (1981) diente der Erfassung der Ängstlichkeit der Mutter wiederum zu allen drei Zeitpunkten.

- Die Skala „Depressivität“ aus dem EMKK von Engfer (1984) erfasst Gefühle der Überforderung und Bedrücktheit in der Beziehung zum Kind sowie Selbstzweifel in der Rolle der Mutter. Die interne Konsistenz der Skala liegt bei .83 (Cronbachs Alpha) (Engfer, 1984).

- $\quad$ Die Skala „Überfürsorge aus Angst“ aus dem EMKK von Engfer (1984) misst Ängste, die um das Wohlergehen des Kindes zentriert sind sowie Tendenzen zur ständigen Kontrolle aus Besorgnis. Die interne Konsistenz der Skala liegt bei .80 (Cronbachs Alpha) (Engfer, 1984).

- Die Skala „Hoffnungslosigkeit“ von Beck (in der deutschen Übersetzung von Krampen, 1979) erfasst den eng mit Depressivität verwandten Aspekt der Hoffnungslosigkeit. Hoffnungslosigkeit wird hier als kognitives System negativer Erwartung über die eigene Person, die Umwelt und das künftige Leben beschrieben.

- $\quad$ Die Skala „Trait Angst“ aus dem STAI von Laux et al. (1981) erfasst Ängstlichkeit im Sinne der zeitstabilen Neigung, Situationen als bedrohlich zu bewerten. Die Skala korreliert hoch mit Neurotizismus- und Depressionsscores. Die Retestreliabilität nach 3,5 Monaten beträgt .81, nach 6 Monaten .68 (Laux et al., 1981).

Die drei Skalen wurden daher pro Untersuchungszeitpunkt nach z-Transformation summiert. Dieser Summenscore wird im folgenden als "Depressivität/Ängstlichkeit“ bezeichnet. 


\section{Erfassung der Kontrollmerkmale}

\subsubsection{Erfassung des kognitiven Entwicklungsstandes}

Für die Beurteilung des kognitiven Entwicklungsstandes der Säuglinge fand eine Ermittlung des „Bayley Mental Developmental Index“ (Bayley, 1993,) zu den drei Erhebungszeitpunkten statt.

Um den kognitiven Entwicklungsstand im Säuglingsalter beurteilen zu können, wird auf internationaler Ebene die "Bayley Mental Scale“ (Bayley, 1993) aufgrund ihrer besten Testgütekriterien (Laucht et al., 1993) als häufigstes Verfahren eingesetzt. Gleichermaßen belegten Laucht et al. (1993) eine vergleichsweise hohe Validität dieses Verfahrens im Rahmen der Mannheimer Säuglingsstudie. Hauptmerkmale des „Bayley Mental Tests“ stellen im ersten Lebensjahr die Items des Bewusstseins des Kindes über seine Umwelt, seine Anpassungsleistung, seine sensorische Reaktionen und die Spanne der Aufmerksamkeit für Stimuli dar (Bayley, 1993). Um das Ziel einer höheren Standardisierung der gesamten Untersuchung erlangen zu können, wurde in Anbetracht an Rennen-Allhoff (1990) die Verwendung eines festen Itemsatzes pro Altersbereich beschlossen. Alle Items wurden einer Prüfung unterzogen, deren Altersplatzierungsrange (Bayley, 1993) das Untersuchungsalter einschloss. Die Vorgaben einer konstanten Abfolge wurden eingehalten (vgl. Pauli-Pott, 2001).

Es kam zu einem Abbruch des Tests, wenn der Verhaltenszustand des Säuglings (Schreien, Einschlafen) keinen angemessenen Untersuchungsverlauf mehr gewährleisten konnte. Es fand dann eine Fortsetzung der Untersuchung bei den folgenden Hausbesuchen statt. Dieses Procedere war im Alter von 8 Monaten lediglich vereinzelt notwendig. Im Alter von 12 Monaten konnten die Untersuchungen ausnahmslos ohne Unterbrechung bzw. Fortsetzung vollzogen werden.

Um eine exakte Testdurchführung zu gewährleisten, wurden zu Beginn einer Erhebungswelle die Untersuchungspersonen hinsichtlich des Vorgehens ausführlich geschult. Um die Genauigkeit und Konsistenz der einzelnen Items zu erhöhen, fand eine Auswertung mit Hilfe von videographischen Mitschnitten der Tests durch die Studienleiterin Dr. Ursula Pauli-Pott statt. 


\subsubsection{Erfassung von kindlichen Koliken, Stilldauer und Stillverhalten, Körpergewicht und Anzahl der Arztbesuche}

Das Kontrollmerkmal kindliche „Koliken“ (Perioden unberuhigbaren Schreiens) wurde anhand des strukturierten Interviews mit der Bezugsperson zum Zeitpunkt des vierten Lebensmonats erfasst. Die Stilldauer und das Stillverhalten, das Körpergewicht und die Anzahl der Arztbesuche wurden zum Zeitpunkt des vierten, achten und zwölften Lebensmonats erfasst. Die Häufigkeit der Koliken konnte mit "keine“, „etwas" und "deutlich" beurteilt werden. Das Stillverhalten bezog sich u.a. darauf, ob das Kind nur gestillt, gestillt und zugefüttert oder nicht gestillt wurde. Bei der Stilldauer konnten die Mütter angeben, ob das Kind zum Zeitpunkt der Befragung noch gestillt wurde bzw. abgestillt wurde. Ebenfalls wurde die Gesamtstilldauer und die Stillund Fütterfrequenz erfragt. Die Anzahl der Arztbesuche schloss die Vorsorgeuntersuchungen aus.

Eine Übersicht über die zeitliche Erfassung der Kontrollmerkmale gibt Tabelle 3.

Tabelle 3: Übersicht der zeitlichen Erfassung der Kontrollmerkmale

\begin{tabular}{|l|l|}
\hline Erfassungszeitpunkt & Kontrollmerkmal \\
\hline 4. Lebensmonat & - Kognitiver Entwicklungsstand \\
& - Kindliche „Koliken“ \\
& - Stilldauer und Stillverhalten \\
& - Körpergewicht \\
& - Anzahl der Arztbesuche \\
\hline 8. Lebensmonat & - Kognitiver Entwicklungsstand \\
& - Stilldauer und Stillverhalten \\
& - Körpergewicht \\
& - Anzahl der Arztbesuche \\
\hline 12. Lebensmonat & - Kognitiver Entwicklungsstand \\
& - Stilldauer und Stillverhalten \\
& - Körpergewicht \\
& - Anzahl der Arztbesuche \\
\hline
\end{tabular}




\subsubsection{Statistische Signifikanz und statistische Analyseverfahren}

Im Folgenden werden zur Bearbeitung der Fragestellungen parametrische und nonparametrische Korrelationskoeffizienten, Chi-Techniken und logistische Regressionsanalysen eingesetzt.

Im Vorfeld fand eine Untersuchung der Verteilung der Variablen hinsichtlich ihrer möglichen deutlichen Abweichungen von einer Normalverteilung statt.

Von statistischer Signifikanz wird konventionsgemäß bei Vorliegen einer maximal $5 \%$ igen Irrtumswahrscheinlichkeit ausgegangen. Das Vorliegen statistischer Trends $(p<0,10)$ wird angegeben.

Alle Berechnungen wurden mit SPSS 11.0 durchgeführt. 


\section{Ergebnisse}

\subsection{Schlaf-Wach-Verhalten}

\subsection{1 Übersicht Schlaf-Wach-Verhalten der Säuglinge}

Das Aufwachen der Säuglinge pro Nacht und die Nächte mit Aufwachepisoden pro Woche sowie die durchschnittliche Schlafdauer pro Nacht ohne Unterbrechung und die Anzahl der Schlafphasen pro Tag wurden mit Hilfe von strukturierten Interviews der Bezugsperson zum Zeitpunkt des 8. und 12. Lebensmonats gewonnen. Hierbei bezogen sich die durchschnittliche Schlafdauer ohne Unterbrechung und die Wachepisoden auf den Zeitraum der Nacht (von 18:00 bis 6:00 Uhr). Die Anzahl der Schlafphasen bezogen sich auf den Tag.

\subsubsection{Schlaf im Alter von 8 Monaten}

Zunächst folgt die Darstellung der Daten der kontinuierlichen Schlafperiode der Säuglinge um damit einen Überblick über das Schlafverhalten ohne Unterbrechung (von 18:00 bis 6:00 Uhr) im Alter von 8 Monaten zu geben. Die durchschnittliche Schlafdauer ohne Unterbrechung pro Nacht beträgt in der untersuchten Stichprobe von 94 Säuglingen 8,32 Stunden. Die kürzeste Schlafdauer liegt bei 2 Stunden pro Nacht, die längste Schlafdauer bei 12 Stunden. Die Standardabweichung der Verteilung beträgt 3,07 Stunden (siehe Abbildung 1). 


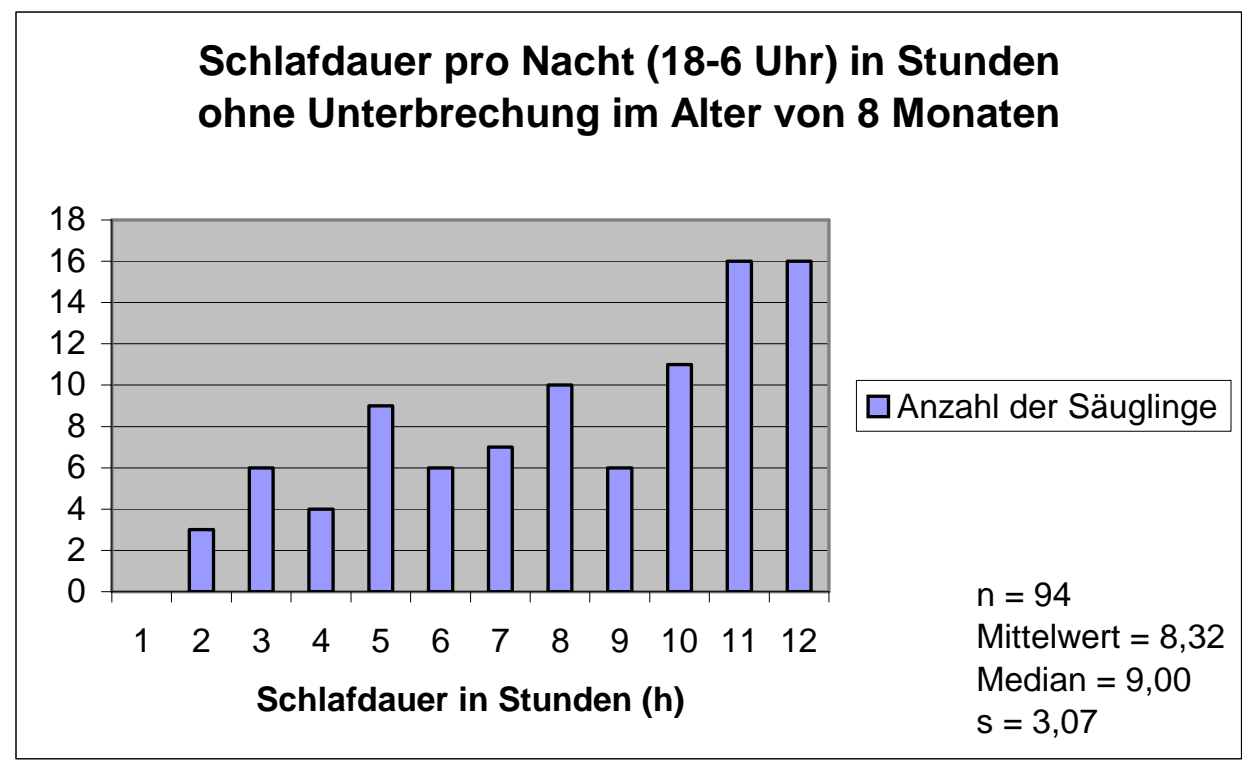

Abbildung 1: Schlafdauer pro Nacht (18:00 bis 6:00 Uhr) in Stunden ohne Unterbrechung

Die Anzahl der Schlafphasen des Kindes pro Tag wurde mit Hilfe des Kurzinterviews der Mutter erfasst und liegt bei 1,97 Schlafphasen ( $n=94$; Median 2,00; $s=0,52$ ).

Das heißt: Die Säuglinge weisen pro Tag im Mittel 2 Schlafperioden auf, wobei die längste Schlafdauer sich im Schnitt auf ein Drittel (circa 8 Stunden) des Tages beläuft.

\subsubsection{Schlafprobleme im Alter von 12 Monaten: Kriteriumsvariablen}

Die Kriteriumsvariablen waren:

a) Anzahl der Nächte mit Aufwachepisoden pro Woche

b) Anzahl der Aufwachepisoden pro Nacht

Es weisen circa $61 \%$ der Säuglinge lediglich 0 bis 1 Wachepisode pro Nacht (18:00 bis 6:00 Uhr) auf, circa $34 \% 2$ oder mehr Wachepisoden pro Nacht (18:00 bis 6:00 Uhr). Für 5 Säuglinge liegen keine Angaben vor (siehe Abbildung 2). 


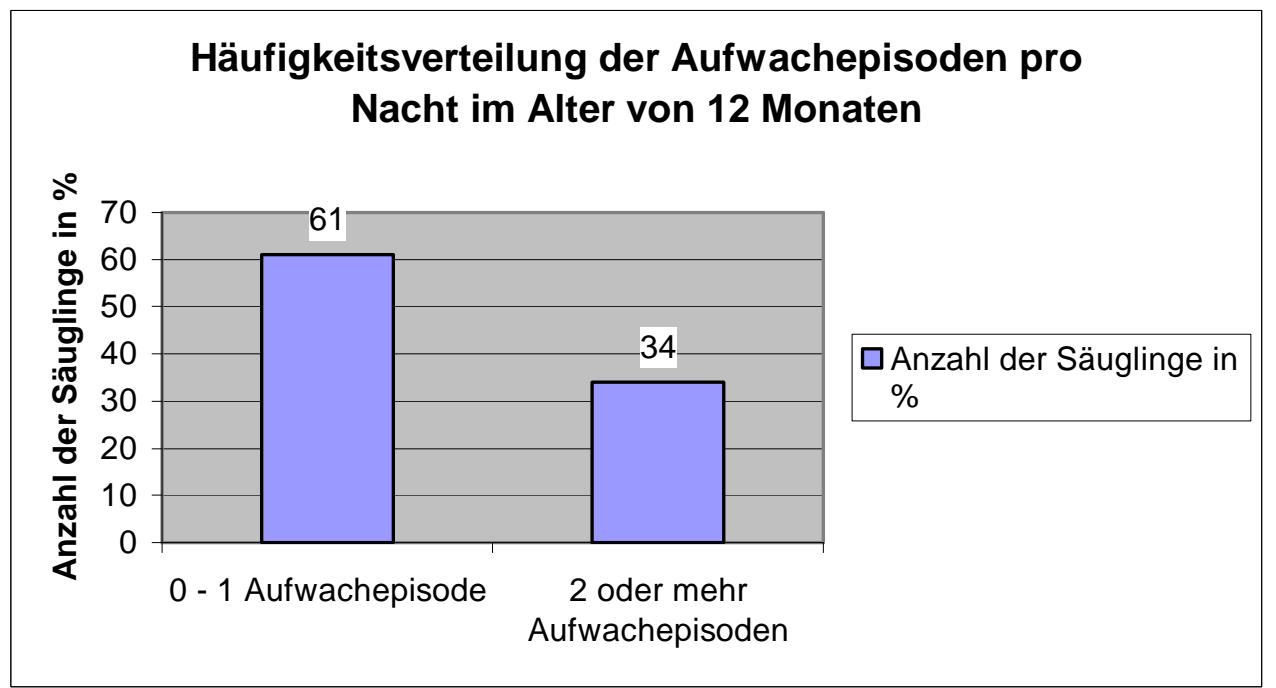

Abbildung 2: Häufigkeitsverteilung der Aufwachepisoden pro Nacht im Alter von 12 Monaten

Bei der Betrachtung der Häufigkeitsverteilung der Nächte pro Woche, in denen Wachepisoden der Säuglinge auftraten, zeigt sich, dass $51 \%$ der Säuglinge an weniger als 2 Nächten pro Woche Durchschlafprobleme aufweisen, $43 \%$ jedoch an 3 oder mehr Nächten der Woche Durchschlafprobleme haben. Für 6 Säuglinge liegen keine Angaben vor (siehe Abbildung 3).

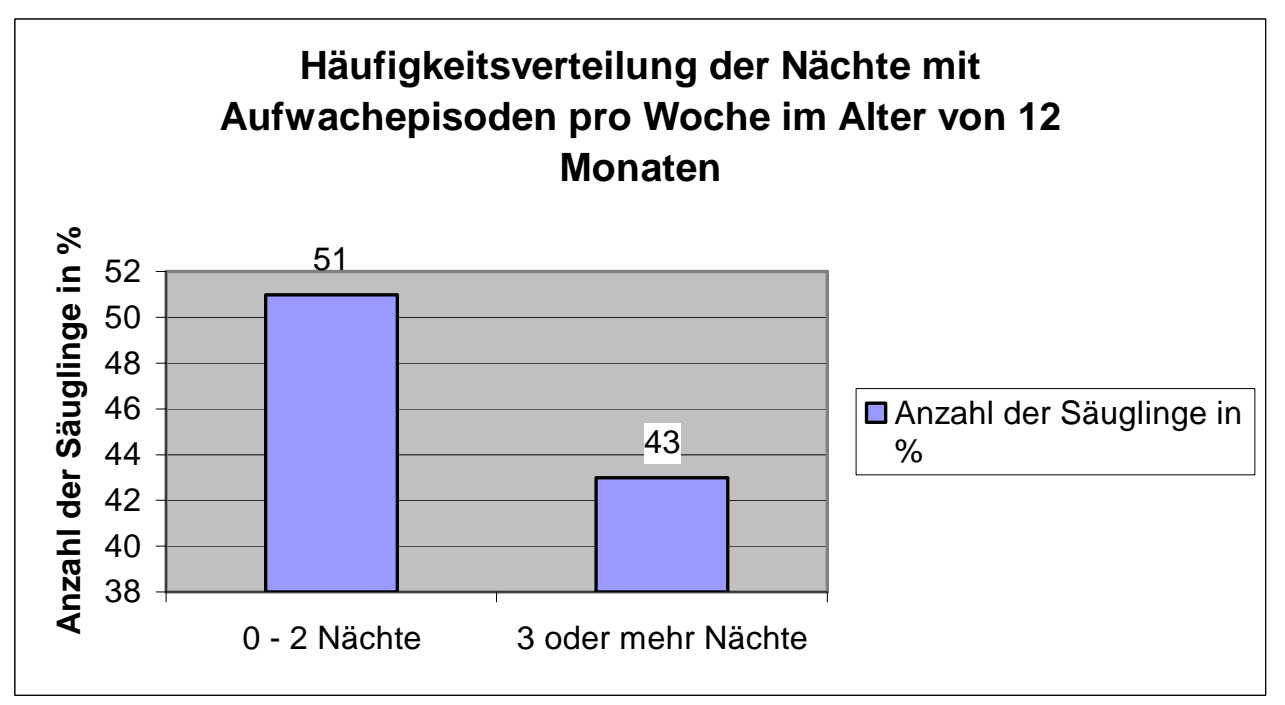

Abbildung 3: Häufigkeitsverteilung der Nächte mit Aufwachepisoden pro Woche im Alter von 12 Monaten

Darüber hinaus zeigt sich bei der Betrachtung der Variablen „Anzahl der Wachepisoden pro Nacht“ und "Anzahl der Nächte pro Woche mit Wachepisoden“ im Chi- 
Quadrat-Test, dass die Säuglinge, welche selten pro Nacht (0 bis 1 Mal) aufwachen auch nur maximal 2 Nächte pro Woche aufwachen. Säuglinge mit mehr als 2 Wachepisoden pro Nacht, wachen auch, auf die Anzahl der Nächte pro Woche bezogen (3 oder mehr Nächte), häufiger auf. 


\subsection{Kontrollmerkmale}

Im nun folgenden Abschnitt stehen die Kontrolle und Mitberücksichtigung von Merkmalen mit möglichen Zusammenhängen zur Schlafentwicklung der Säuglinge während der drei Untersuchungszeitpunkte (4., 8., 12. Monat) im Vordergrund. Diese setzen sich aus „Arztkonsultationen“, „Körpergewicht des Säuglings“, „Stilldauer und Stillverhalten“, der „kognitiven Entwicklung des Säuglings“ und den „Koliken im ersten Lebensjahr" zusammen.

In Abschnitt 5.3 erfolgt die Hypothesenprüfung der Hauptmerkmale "Temperament“ sowie „mütterlicher Depressionsneigung und Ängstlichkeit“ zum Zeitpunkt des 4., 8. und 12. Lebensmonats.

In beiden Abschnitten soll geprüft werden, ob zum Zeitpunkt der Untersuchung im 4., 8. und 12. Monat die genannten Merkmale Vorhersagen des Schlafverhaltens der Kinder im Alter von 12 Monaten geben können.

\subsubsection{Arztkonsultationen}

In diesem Abschnitt sollen die Häufigkeit der Arztbesuche der Säuglinge im Alter von 4, 8 und 12 Monaten näher betrachtet werden und mögliche Korrelationen mit dem Schlafverhalten überprüft werden.

Im Alter von 4 Monaten setzt sich die Stichprobe aus insgesamt 98 Säuglingen zusammen. Wie aus Abbildung $4 \mathrm{zu}$ ersehen ist, liegt der Mittelwert der Anzahl der Arztbesuche ohne Vorsorgeuntersuchungen bis zum 4. Lebensmonat bei 1,29 ( $\mathrm{n}=$ 98; Median =1,0, $s=1,59$ ). Die minimalste Anzahl liegt bei null Besuchen, das Maximum beträgt 9 Besuche in den ersten 4 Lebensmonaten (siehe Abbildung 4). Fast analog verhält sich die Untersuchung der Arztbesuche vom 4. bis zum 8. Lebensmonat. Hierbei können 93 Säuglinge berücksichtigt werden. Der Mittelwert der Arztbesuche liegt in diesem Zeitraum bei 1,27 $(n=93$; Median =1,00; $s=1,87)$. Das Minimum beträgt hier ebenfalls null Arztbesuche, der Spitzenreiter verzeichnet hier allerdings 15 Konsultationen (siehe Abbildung 5). Für den Zeitraum vom 8. bis zum 12. Monat können 92 Säuglinge berücksichtigt werden. Hier zeigt sich ein leichter Anstieg der Arztbesuche ohne Vorsorgeuntersuchungen im Vergleich zu den Vormona- 
ten. Der Mittelwert beträgt 1,58 Arztbesuche ( $n=92$; Median = 1,00; $s=1,77)$. Die minimalste Anzahl liegt hier bei null Besuchen, das Maximum bei 9 Arztbesuchen (siehe Abbildung 6).

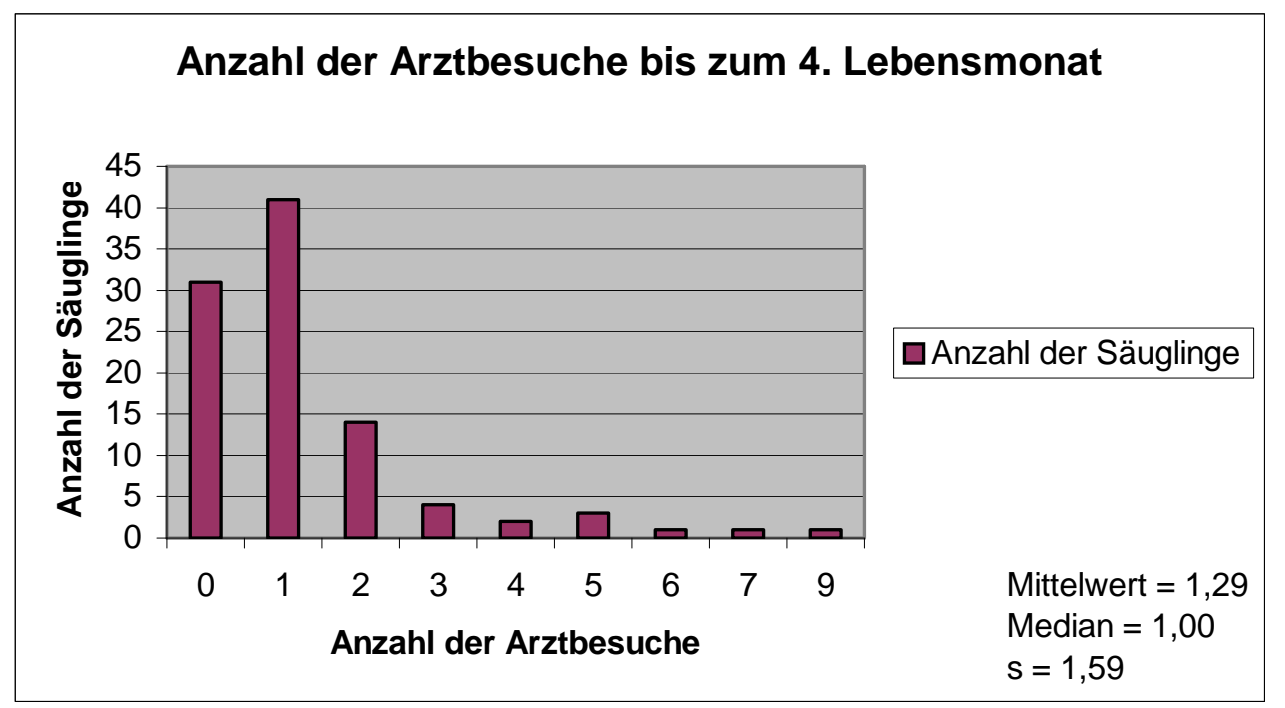

Abbildung 4: Anzahl der Arztbesuche der Säuglinge bis zum 4. Lebensmonat

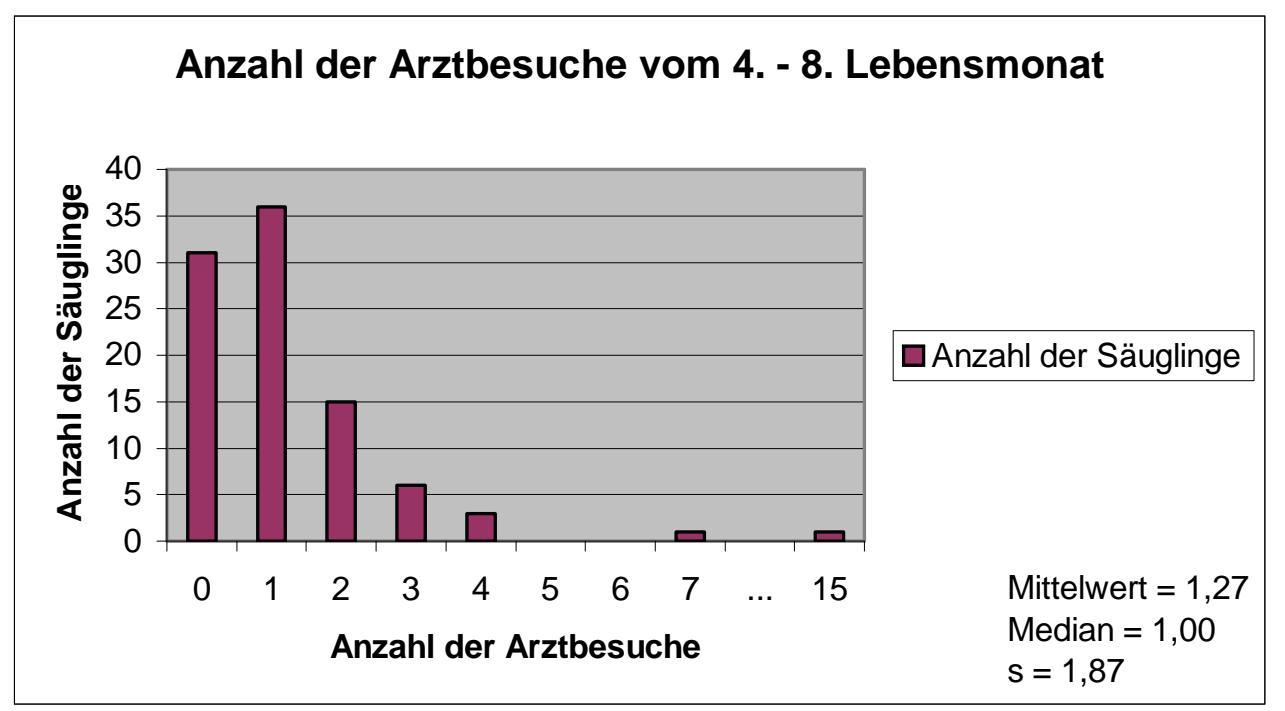

Abbildung 5: Anzahl der Arztbesuche der Säuglinge vom 4. bis 8. Lebensmonat 


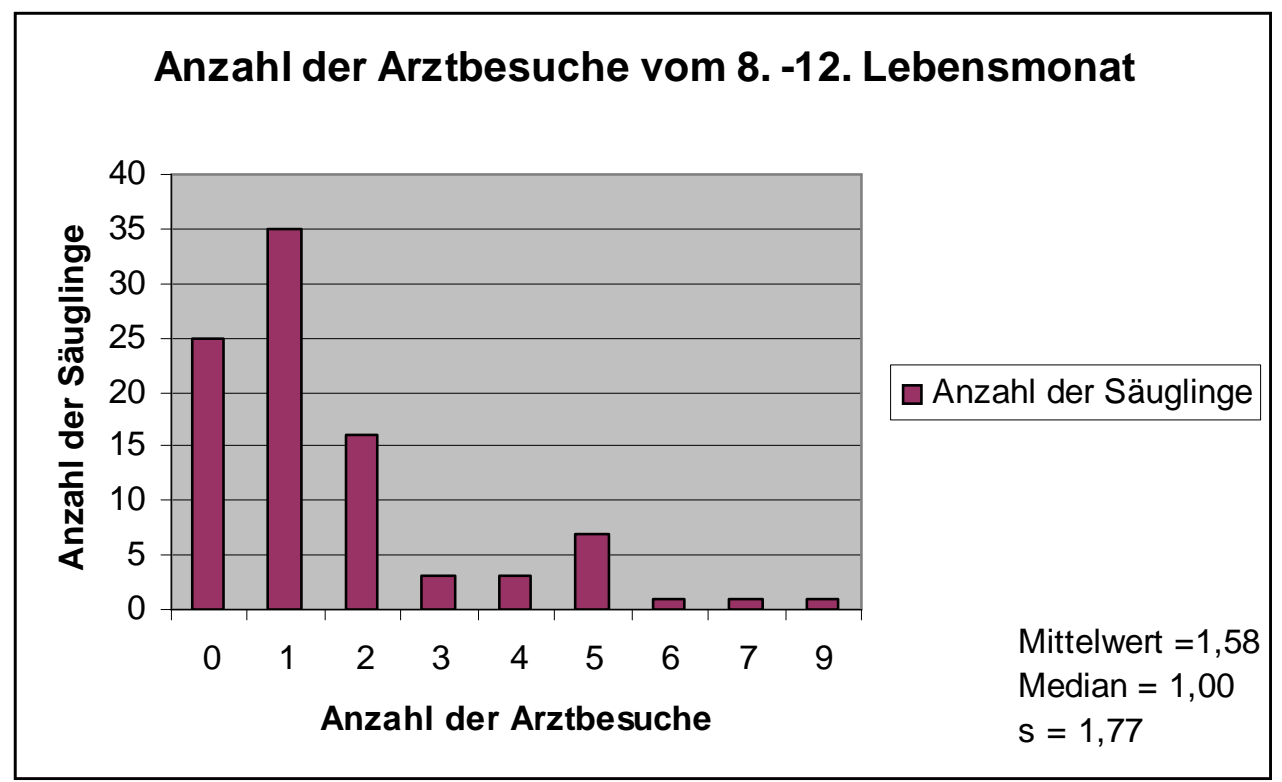

Abbildung 6: Anzahl der Arztbesuche vom 8. bis 12. Lebensmonat

\subsubsection{Korrelation zwischen Arztbesuchen und Schlafverhalten}

Im Allgemeinen kann die Häufigkeit der Arztbesuche als Parameter des Wohlergehens bzw. des Gesundheitszustandes des Säuglings angenommen werden. Im folgenden Abschnitt soll auf mögliche Zusammenhänge zwischen der Anzahl der Arztbesuche und dem Schlafverhalten des Kindes im Alter von 12 Monaten eingegangen werden.

Im Hinblick auf die Arztbesuche bis zum 4. Lebensmonat kann keine signifikante Korrelation mit den nächtlichen bzw. wöchentlichen Aufwachepisoden im Alter von 12 Monaten aufgezeigt werden.

Bei den Arztbesuchen zwischen dem 4. und 8. Lebensmonat lassen sich allerdings in beiden Bereichen sowohl in der Anzahl der nächtlichen Aufwachepisoden als auch in der Häufigkeit der Nächte mit Aufwachepisoden pro Woche im Alter von 12 Monaten statistisch signifikante Korrelationen aufweisen. Bei einer Signifikanz von $p<0,014$ zeigt sich bei den Kindern, welche zwei oder mehrere Male zwischen dem 4. und 8. Lebensmonat einen Arztbesuch aufweisen, eine deutlich höhere Anzahl an Wachepisoden pro Nacht im Alter von 12 Monaten als bei Kindern, die lediglich einmal bzw. keinmal den Arzt in diesem Zeitraum aufgesucht haben (siehe Abbildung 7). 


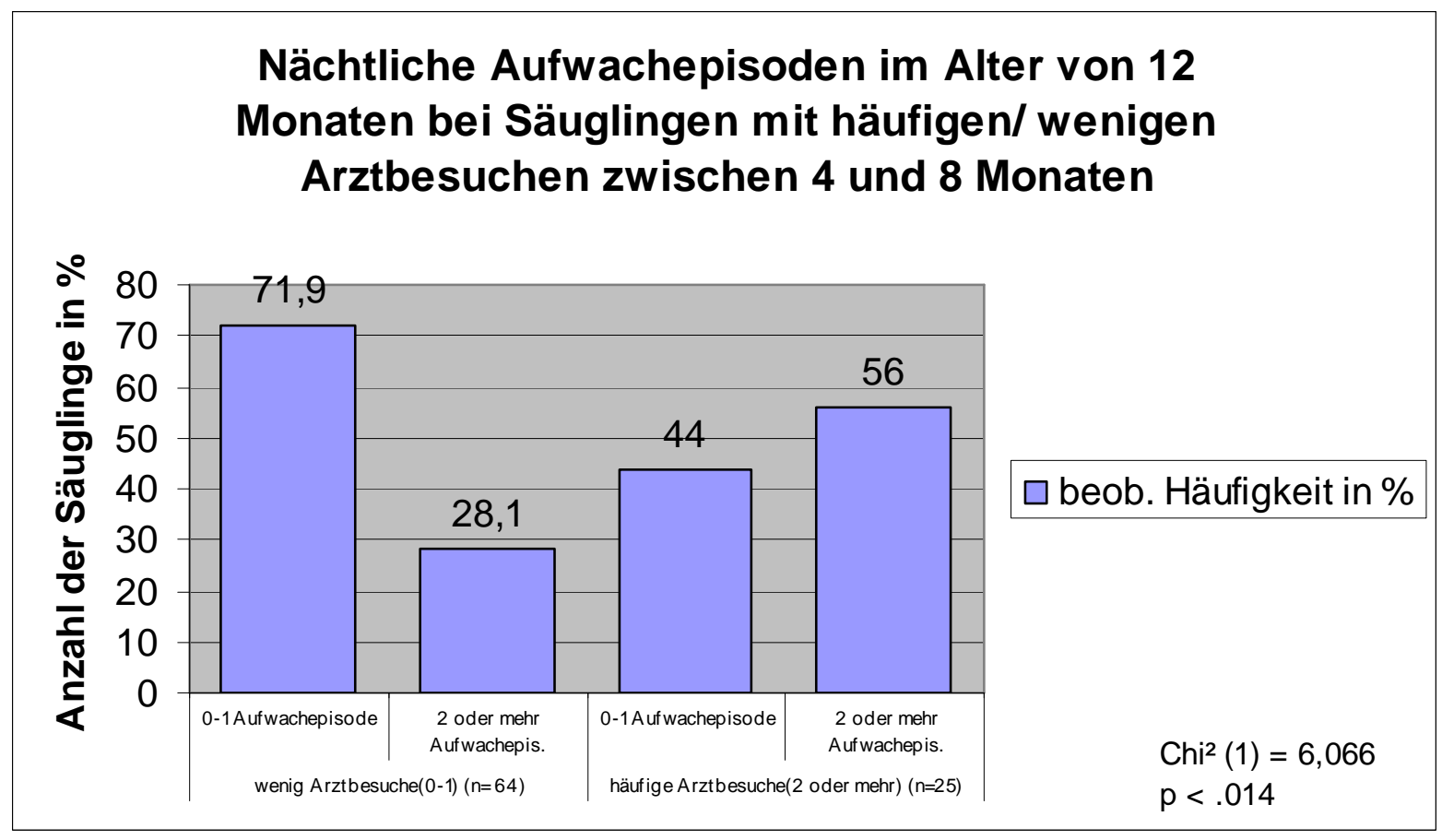

Abbildung 7 : Nächtliche Aufwachepisoden im Alter von 12 Monaten bei Säuglingen mit häufigen/wenigen Arztbesuchen zwischen 4 und 8 Monaten

Dieses Ergebnis wird bei der Betrachtung der Häufigkeit der Wachepisoden pro Woche im Alter von 12 Monaten gefestigt. Bei einem Signifikanzniveau von $p<0,003$ zeigt sich, dass Kinder mit häufigen Arztbesuchen (2 oder mehr) zwischen dem 4. und 8. Lebensmonat auch in der wöchentlichen Aufwachstatistik im 12. Lebensmonat höhere Werte gegenüber Kindern mit wenigen oder keinen Arztbesuchen ( 0 bis 1) zum Zeitpunkt zwischen 4. und 8. Lebensmonat aufweisen (siehe Abbildung 8). 


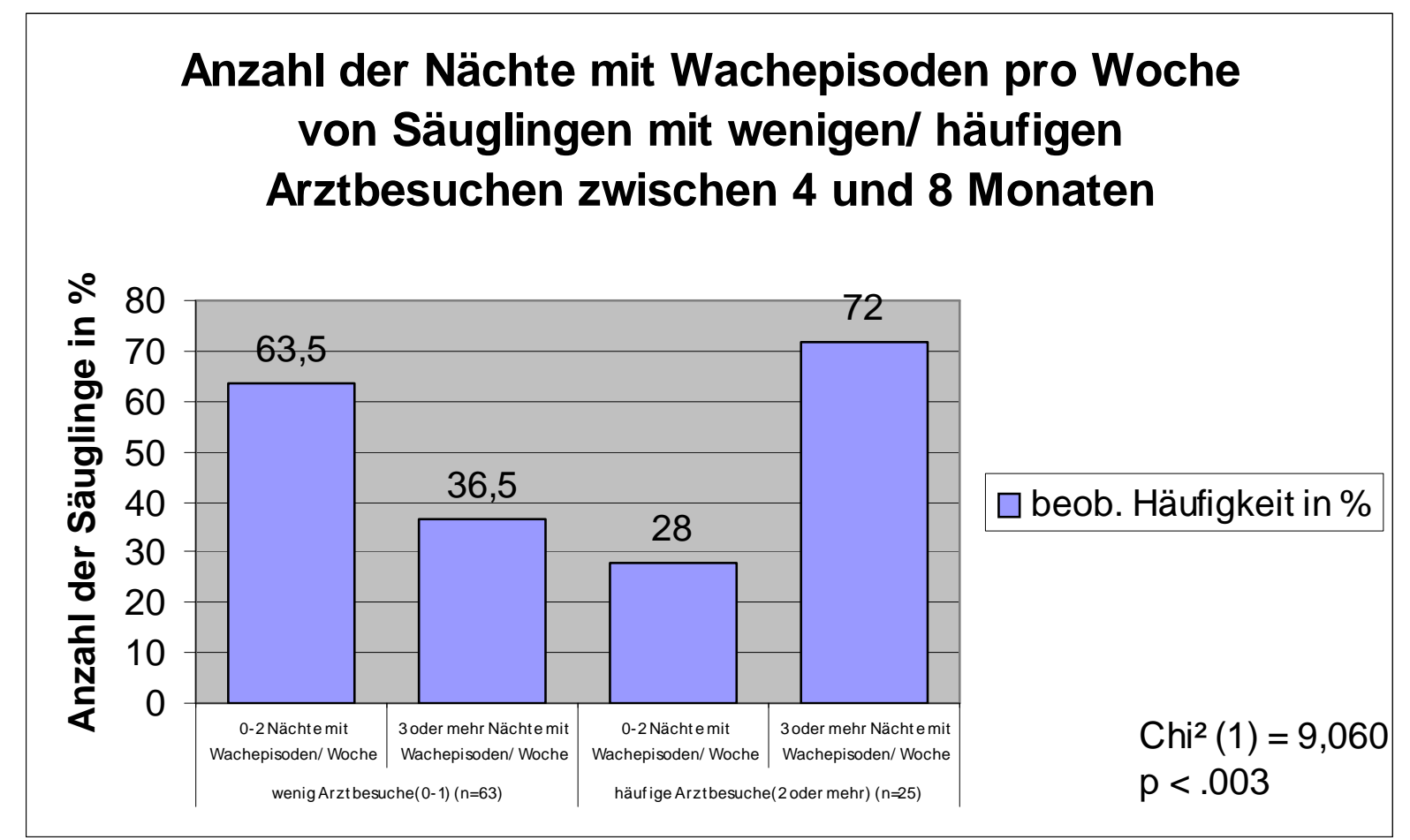

Abbildung 8: Anzahl der Nächte mit Wachepisoden pro Woche von Säuglingen im Alter von 12 Monaten mit wenigen/häufigen Arztbesuchen zwischen 4 und 8 Monaten

Bei der Betrachtung der Arztbesuche der Säuglinge im Alter von 8 bis 12 Monaten mit den nächtlichen Wachepisoden und der Häufigkeit der Wachepisoden pro Woche im Alter von 12 Monaten kann keine weitere signifikante Korrelation festgestellt werden.

Somit ist ein signifikanter Zusammenhang zwischen den Arztbesuchen in der Periode zwischen dem 4. und 8. Monat und den Aufwachepisoden sowohl auf die Nacht als auch auf die Woche bezogen im Alter von 12 Monaten aufzeigbar.

\subsubsection{Körpergewicht des Säuglings}

Die Beurteilung des Körpergewichtes zum Zeitpunkt der Geburt sowie im fortschreitenden Entwicklungsverlauf kann mit Hilfe der Perzentilenkurven aus den Wachstumskurven objektivierbar durchgeführt werden. Das Ergebnis ermöglicht das Aufzeigen möglicher Entwicklungs- und Reifungsverzögerungen. In diesem Kapitel soll das Körpergewicht der Säuglinge im Verlauf der ersten 12 Lebensmonate betrachtet werden. Hierzu erfolgte eine Gewichtsmessung bei der Geburt sowie bei den Vor- 
sorgeuntersuchungen U3 (4. bis 6. Lebenswoche), U5 (6. bis 7. Lebensmonat) und U6 (10. bis 12. Lebensmonat).

Des Weiteren werden mögliche Korrelationen zwischen dem Körpergewicht der Säuglinge und deren Schlafeigenschaften im Alter von 12 Monaten untersucht.

\subsubsection{Körpergewicht zum Zeitpunkt der Geburt und der U3-, U5- und U6- Untersuchung}

Das mittlere Geburtsgewicht der Säuglinge der Stichprobe liegt bei 3415 g. Das geringsten Geburtsgewicht beträgt 2500 g, das Höchstgewicht 4650 g. Die Standardabweichung der Verteilung liegt bei $s=441,15$. ( $n=98$; Median 3400 ; $s=441,15$ ). Abbildung 9 zeigt einen Überblick der Geburtsgewichte aus der gesamten Stichprobe.

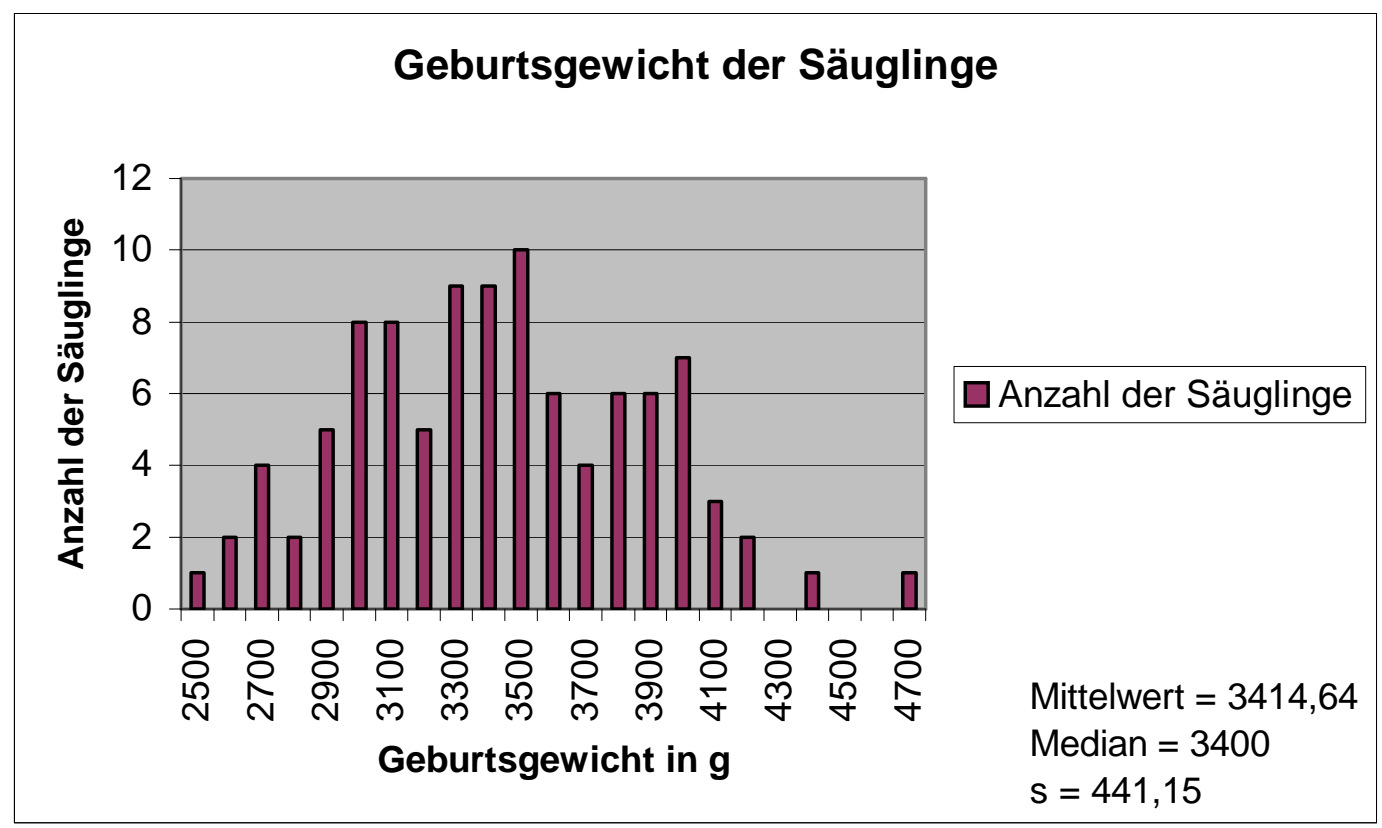

Abbildung 9: Geburtsgewicht der Säuglinge

Zum Zeitpunkt der U3-Untersuchung, welche im Alter von 4 bis 6 Lebenswochen durchgeführt wird, liegt das Durchschnittsgewicht der Säuglinge bei $4474 \mathrm{~g}$. Das geringste Gewicht beträgt bei dieser Untersuchung $3280 \mathrm{~g}$, das höchste $6420 \mathrm{~g}$. Die Standardabweichung der Verteilung liegt bei $s=616,91(n=97$; Median = 4480; $s=$ $616,91)$. 
Im 6. bis 7. Lebensmonat erfolgt erneut eine Gewichtsmessung im Rahmen der U5Vorsorgeuntersuchung. Hierbei liegt der Mittelwert des Körpergewichtes bei $7974 \mathrm{~g}$. Das leichteste Kind bringt es zu diesem Zeitpunkt auf 5590 g, der Höchstwert beträgt beachtliche $11260 \mathrm{~g}$. Die Standardabweichung der Verteilung liegt bei $\mathrm{s}=1084,27$ ( $\mathrm{n}=94 ;$ Median = 7730; $\mathrm{s}=1084,27)$.

Die letzte Gewichtsmessung im Rahmen dieser Studie wird im Alter von 10-12 Lebensmonaten bei der U6-Vorsorgeuntersuchung durchgeführt. Das durchschnittliche Gewicht der Säuglinge liegt hier bei $9668 \mathrm{~g}$, bei einem Minimum von $7500 \mathrm{~g}$ und einem Maximum von $12700 \mathrm{~g}$. Die Standardabweichung der Verteilung liegt bei $\mathrm{s}=$ 1196,42 ( $\mathrm{n}=90 ;$ Median = 9535; $\mathrm{s}=1196,42)$.

\subsubsection{Korrelation des Schlafverhaltens mit dem kindlichen Körperge- wicht}

Fast ausnahmslos lassen sich signifikante Korrelationen des körperlichen Reifezustandes, ablesbar am Körpergewicht des Säuglings, mit dessen nächtlicher Aufwachhäufigkeit im Alter von 12 Monaten finden. Schon beim Geburtsgewicht zeigt sich die signifikant negative Korrelation zur nächtlichen Aufwachhäufigkeit im 12. Lebensmonat. Bei einem Signifikanzniveau von $p<0,017$ wird eine negative Korrelation von $r=-0,247$ deutlich. Dies wiederum macht einen deutlichen Zusammenhang zwischen dem Geburtsgewicht und den Aufwachepisoden im Alter von 12 Monaten sichtbar. Je niedriger das Gewicht des Kindes zum Zeitpunkt der Geburt ist, umso häufiger wacht es später (im 12. Lebensmonat) pro Nacht auf. Gleiches lässt sich bei der Betrachtung der Korrelation beider Merkmale zum Zeitpunkt der Untersuchung U3 (4. bis 6. Lebenswoche) und U6 (10. bis 12. Lebensmonat) aufzeigen. Zu beiden Zeitpunkten zeigen sich negative Korrelationen (bei U3 $r=-0,205$; bei U6 $r=-0,215$ ) bei einem Signifikanzniveau von < 5\%. Lediglich bei der Vorsorgeuntersuchung U5 (6.-7. Lebensmonat) lässt sich kein signifikantes Ergebnis erbringen (siehe Tabelle 4).

Zusammenfassend kann anhand dieser Daten ein klarer Zusammenhang zwischen niedrigerem Körpergewicht im gesamten ersten Lebensjahr und mangelndem nächtlichen Durchschlafverhalten des Kindes im Alter von 12 Monaten aufgezeigt werden. 
Ein signifikantes Ergebnis bezüglich Körpergewicht und Anzahl der Nächte pro Woche mit Wachepisoden im Alter von 12 Monaten kann zu keinem Untersuchungszeitpunkt nachgewiesen werden (siehe Tabelle 4).

Tabelle 4: Pearson Rangkorrelationskoeffizienten der Körpergewichte des Säuglings mit der nächtlichen und wöchentlichen Aufwachhäufigkeit im Alter von 12 Monaten

\begin{tabular}{|l|r|r|}
\hline & Aufwachen pro Nacht & $\begin{array}{r}\text { Nächte mit Wachepisoden pro } \\
\text { Woche }\end{array}$ \\
\hline Geburtsgewicht & $r=-0,247$ & $r=-0,062$ \\
& $p<0,017$ & $p=n s$ \\
\hline Gewicht bei U3 & $r=-0,205$ & $r=-0,027$ \\
& $p<0,049$ & $p=n s$ \\
\hline Gewicht bei U5 & $r=-0,181$ & $r=0,024$ \\
& $p=n s$ & $p=n s$ \\
\hline Gewicht bei U6 & $r=-0,215$ & $r=-0,088$ \\
& $p<0,042$ & $p=n s$ \\
\hline
\end{tabular}

Anmerkung: Mitgeteilt sind punktbiseriale Korrelationskoeffizienten und Irrtumswahrscheinlichkeit (p); ns: nicht statistisch signifikant

\subsubsection{Stilldauer und Stillverhalten}

Im vorangegangenen Abschnitt konnte ein Zusammenhang zwischen dem geringen Körpergewicht und einer vermehrten nächtlichen Aufwachfrequenz aufgezeigt werden. Nun sollen mögliche Assoziationen des Stillverhaltens und der Stilldauer mit dem Schlafverhalten betrachtet werden.

Die Daten über Stilldauer und Stillverhalten wurden anhand der Kurzinterviews mit den Bezugspersonen zum Zeitpunkt des 4., 8. und 12. Lebensmonats erfasst.

\subsubsection{Dauer der Stillperiode}

Die untersuchte Stichprobe weist zum einen Kinder auf, welche nur kurz (ein bis zwei Wochen) gestillt wurden. Demgegenüber beläuft sich das Höchstmaß an Stilldauer 
auf mehr als 48 Wochen. Das heißt, dass insgesamt 12 Mütter beim letzten Befragungstermin im Alter von 12 Monaten noch stillten. Die Durchschnittsdauer der Stillperiode beträgt 27,46 Wochen. Die Standardabweichung der Verteilung liegt bei $13,49(n=91$, Median $=26,0 ; s=13,49)$ (siehe Abbildung 10).

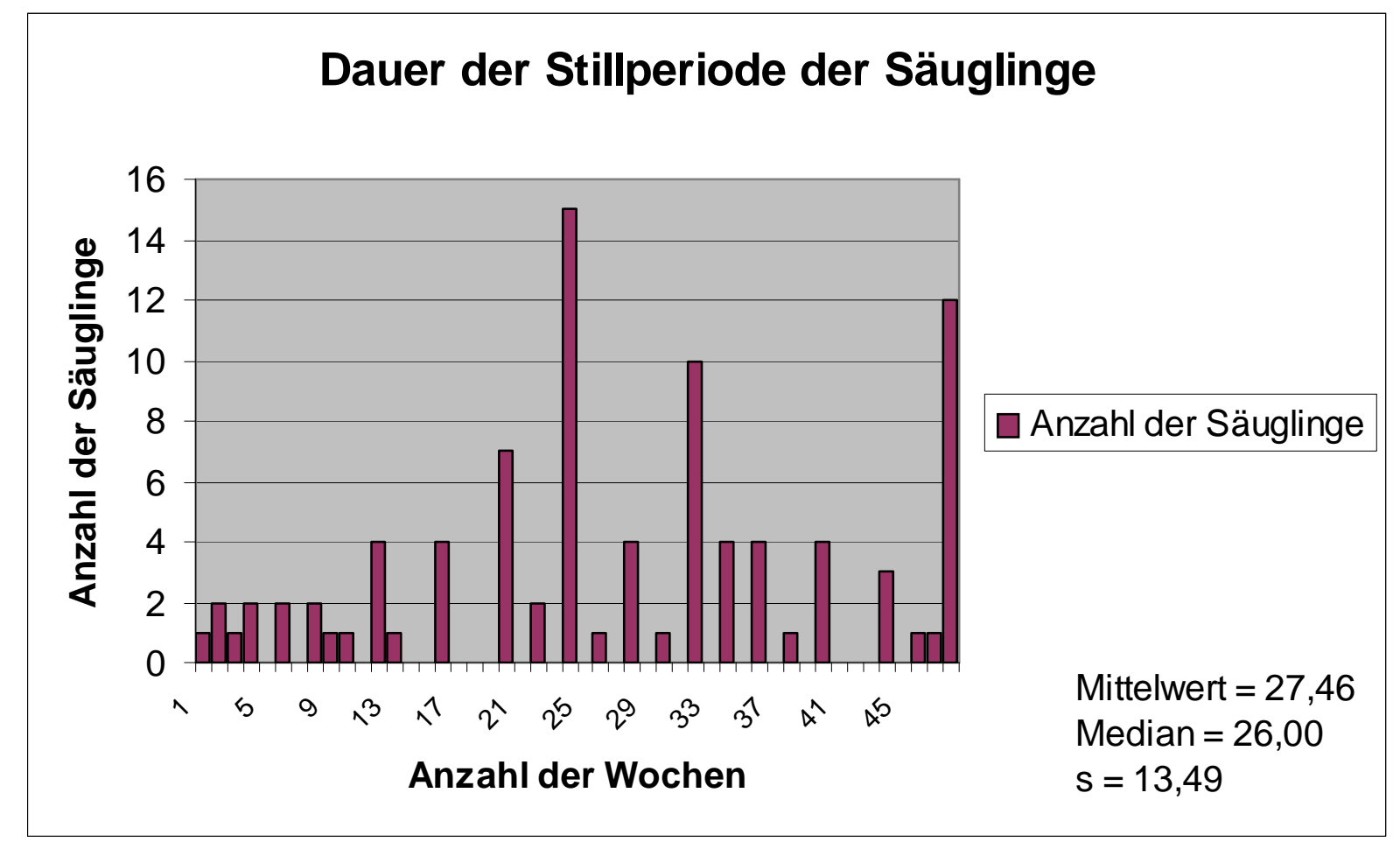

Abbildung 10: Dauer der Stillperiode der Säuglinge

\subsubsection{Untersuchung der Korrelation zwischen Stilldauer, Gewicht und Schlafeigenschaften}

Die Untersuchung möglicher Zusammenhänge zwischen den Schlafeigenschaften und der Stilldauer wird mit Hilfe der Pearson-Produkt-Momentkorrelation durchgeführt. Eine Signifikanz kann sowohl in Bezug auf die nächtlichen Wachepisoden als auch auf die Anzahl der Nächte mit Wachepisoden pro Woche im Alter von 12 Monaten nicht aufgezeigt werden.

Ähnlich verhält es sich bei der Betrachtung der Korrelation zwischen kindlichem Körpergewicht und Stilldauer, welches mit Hilfe des Spearman-Rho-Verfahrens ausgewertet wurde. Eine signifikante Beziehung kann zu keinem Zeitpunkt verzeichnet werden. 
Folglich stellt sich die Länge der Stillperiode hinsichtlich des kindlichen Schlafverhaltens in der untersuchten Stichprobe als nicht entscheidend dar.

\subsubsection{Kognitive Reife des Säuglings}

Das folgende Kapitel befasst sich mit der Betrachtung des kognitiven Entwicklungsstandes respektive der geistigen Reife des Säuglings. Das Ziel der Untersuchung besteht in der Prüfung einer möglichen Beziehung zwischen der geistigen Reife und dem Schlafverhalten des Säuglings im Alter von 12 Monaten. Die Merkmale des kindlichen Bewusstseins über seine Umwelt, seine Anpassungsleistung, seine sensorischen Reaktionen sowie die Spanne der Aufmerksamkeit für Stimuli wurden zu den drei Erhebungszeitpunkten (4, 8, 12 Monate) durch den „Bayley Mental Test“ durchgeführt (Bayley, 1993).

\subsubsection{Korrelation zwischen kognitiver Entwicklung und Schlafverhalten}

Eine signifikante Korrelation zwischen geistiger Reife und der Anzahl der nächtlichen Aufwachepisoden im 12. Lebensmonat ist tendenziell nur zum Zeitpunkt des 8. Lebensmonats zu erkennen. Bei einem Signifikanzniveau von $p<0,065$ ist eine negative Korrelation nachzuweisen ( $p=0,065 ; r=-0,195)$. Dies bedeutet, dass Säuglinge mit einem geringerem Stand geistiger Reife im Alter von 8 Monaten während der nächtlichen Schlafphase im Alter von 12 Monaten vermehrte Wachepisoden aufweisen. Zum Untersuchungszeitpunkt von 4 und 12 Monaten kann kein bedeutsames Ergebnis gezeigt werden (4 Monate: $p=n s ; r=0,058 ; 12$ Monate: $p=n s ; r=-$ 0,013).

Ein hochsignifikantes Ergebnis mit $p<0,001$ ergibt die Korrelationsuntersuchung des kognitiven Entwicklungsstandes im Alter von 12 Monaten mit der Anzahl der Nächte mit Wachepisoden pro Woche im Alter von 12 Lebensmonaten. Es zeigt sich eine positive Korrelation ( $r=0,327)$. Das heißt: Säuglinge mit hoher kognitiver Entwicklung leiden im Alter von 12 Monaten bezogen auf die Woche häufiger an Durchschlafproblemen als Säuglinge mit geringer kognitiver Entwicklung zu diesem Zeitpunkt. Dieses Ergebnis stellt sich bei der Untersuchung der kognitiven Reife im 12. 
Monat signifikant dar. Bei der Untersuchung der kognitiven Reife im Alter von 4 und 8 Monaten zeigt sich hingegen kein signifikanter Zusammenhang (4 Monate: $p=n s ; r$ $=-0,024 ; 8$ Monate: $p=n s ; r=-0,058)$.

\subsubsection{Koliken bis zum Alter von 4 Monaten}

Das Auftreten von Koliken im Verlaufe der ersten Lebensmonate und aktuelle Koliken (zum Zeitpunkt des 4. Lebensmonats) wurde bei den Säuglingen der Stichprobe im 4. Lebensmonat mit Hilfe des Kurzinterviews der Bezugsperson ermittelt. Koliken bedeuten in diesem Zusammenhang „Perioden unberuhigbaren Schreiens“. Im weiteren Verlauf sollen die Zusammenhänge zwischen dem Auftreten von Koliken und dem Schlafverhalten im Alter von 12 Monaten untersucht werden.

\subsubsection{Häufigkeit von Koliken in der Stichprobe}

Die Häufigkeit der Koliken (Perioden unberuhigbaren Schreiens) wurde mittels Befragung der Mutter für zwei Zeitabschnitte erfasst. Ein Zeitabschnitt bezog sich aktuell auf den vierten Lebensmonat, der andere auf die vorangegangene Zeit (Geburt bis zum 4. Lebensmonat). Die Mütter konnten je nach Auftreten der Koliken die Häufigkeit mit keine (1), etwas (2) und deutliche (3) Koliken beschreiben. Zeitlich gesehen kann man über die 98 Säuglinge der Stichprobe sagen, dass das Ausmaß der Koliken bis zum 4. Lebensmonat deutlich zurückgeht. Wie aus Abbildung 11 ablesbar ist, klagen $51 \%$ der Mütter über gelegentliche bis deutliche Koliken ihrer Kinder in den vorangegangenen 4 Monaten (Geburt bis 4. Lebensmonat). 49 \% der Befragten verneinen Koliken ihrer Säuglinge in dieser Zeit. 


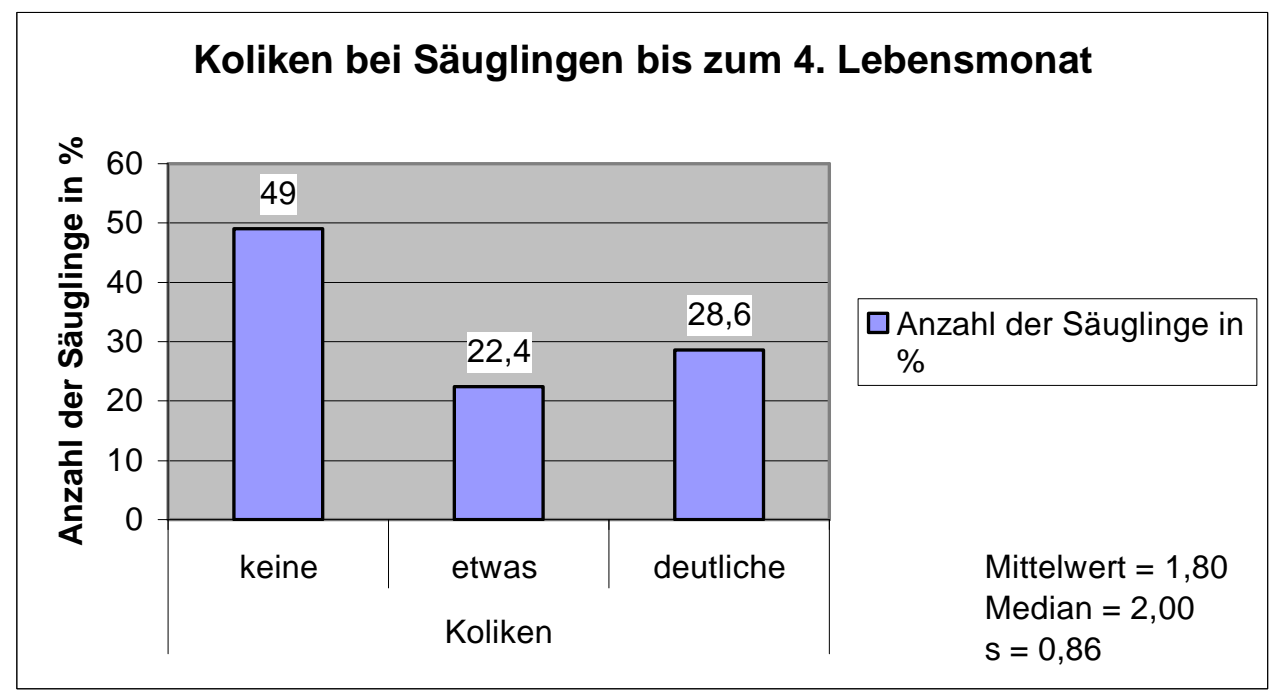

Abbildung 11: Koliken bei Säuglingen bis zum 4. Lebensmonat

Im vierten Lebensmonat berichten lediglich noch $17 \%$ der Mütter aktuell von gelegentlich bis häufigen Koliken. 83 \% der Säuglinge sind zum Zeitpunkt der Befragung kolikfrei (siehe Abbildung 12).

Darüber hinaus verringert sich die Prozentzahl der Säuglinge mit häufigen Koliken im Verlaufe der ersten Lebensmonate bis zum vierten Lebensmonat von $29 \%$ auf lediglich $3 \%$ (vgl. hierzu Abbildung 11 \& Abbildung 12).

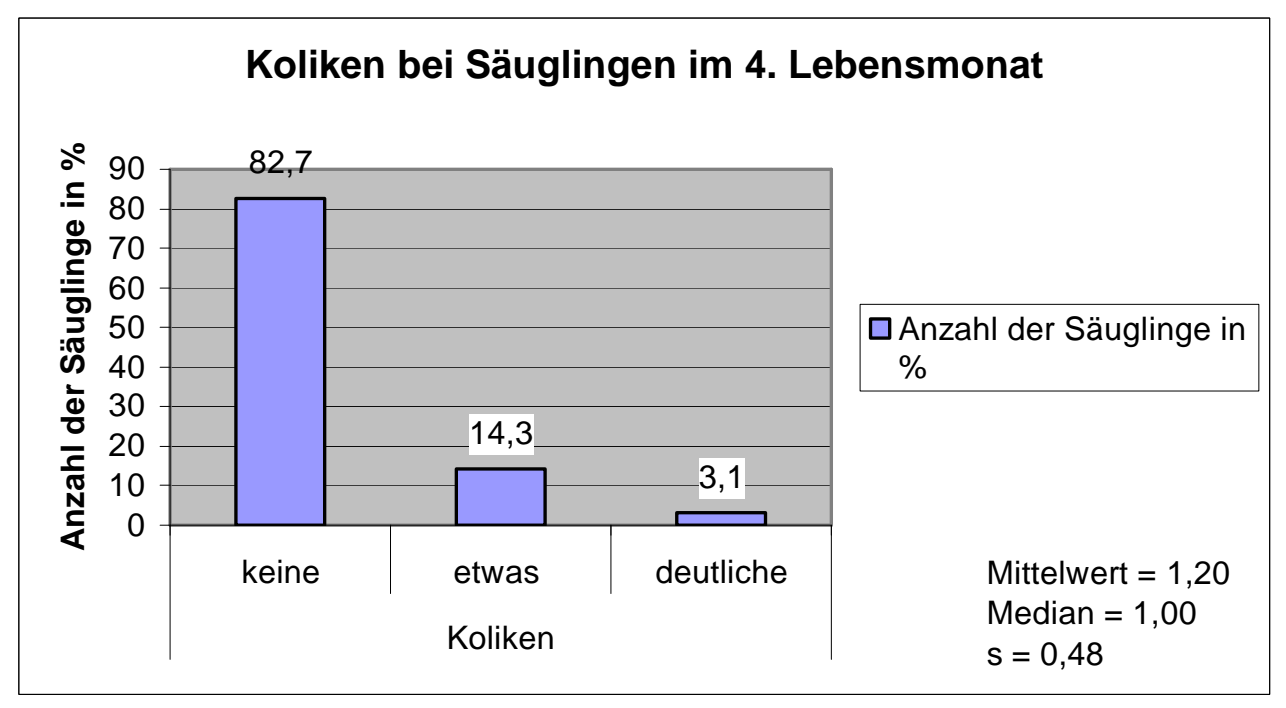

Abbildung 12: Koliken bei Säuglingen im 4. Lebensmonat 


\subsubsection{Zusammenhänge zwischen Kolikausprägung und Schlafverhalten}

Für den Zeitraum von der Geburt des Säuglings bis zur Befragung der Mutter im 4. Lebensmonat zeigt sich im Chi²-Test, bezogen auf die Anzahl der Nächte mit Wachepisoden pro Woche im Alter von 12 Monaten, ein signifikantes Ergebnis mit $\mathrm{p}<$ $0,004\left(\mathrm{Chi}^{2}(1)=8,121 ; \mathrm{p}<0,004\right)$. Wie aus Abbildung 13 ersichtlich ist, liegt die Anzahl der Säuglinge ohne bzw. mit wenigen Koliken bis zum 4. Lebensmonat, welche oft (>3 Nächten) pro Woche im Alter von 12 Monaten aufwachen, deutlich unter der erwarteten Häufigkeit (beob. Häufigkeit: 24 (36,4 \%) vs. erw.: Häufigkeit: 30,1 (45,6 $\%)$ ). Demgegenüber übersteigt die Anzahl der Kinder mit deutlichen Koliken und zahlreichen (>3 Nächte) Aufwachepisoden pro Woche im Alter von 12 Monaten die erwartete Häufigkeit um gut ein Drittel (beob. Häufigkeit:18 (69,2\%) vs. erw.: Häufigkeit: $11,9(45,7 \%))$.

So zeigt sich eine höhere Aufwachhäufigkeit pro Woche im Alter von 12 Monaten bei Säuglingen mit deutlichen Koliken bis zum 4. Lebensmonat im Gegensatz zu den Säuglingen, welche nur wenige bzw. keine Koliken zu diesem Zeitpunkt haben (siehe Abbildung 13).

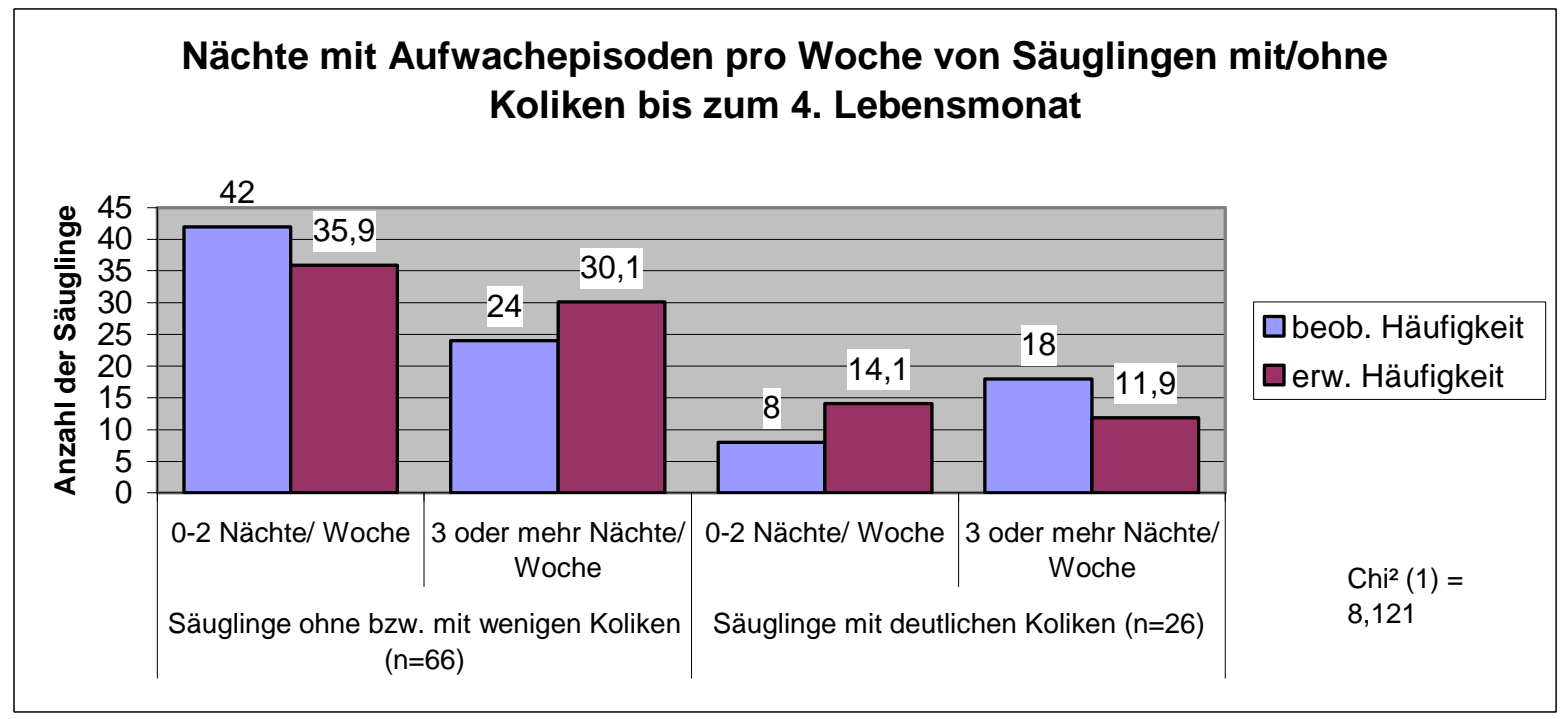

Abbildung 13: Nächte mit Aufwachepisoden pro Woche im 12. Lebensmonat von Säuglingen mit/ohne Koliken bis zum 4. Lebensmonat

Zum Untersuchungszeitpunkt im 4. Lebensmonat ist das oben genannte Ergebnis in Bezug auf die Nächte mit Aufwachepisoden pro Woche im 12. Lebensmonat nicht 
mehr aufrecht zu erhalten. Es kann im Chi²-Test kein signifikantes Ergebnis erreicht werden.

Bei der Betrachtung der Koliken in den ersten Lebensmonaten (Geburt bis 4. Lebensmonat) in Bezug auf die nächtliche Aufwachfrequenz im 12. Monat lässt sich mit Hilfe des Chi²-Tests kein signifikantes Ergebnis aufzeigen.

Erst im 4. Lebensmonat stellt sich mittels des Chi²-Tests $\left(\right.$ Chi $^{2}(1)=4,696$; $)$ eine signifikante Verbindung $(\mathrm{p}<0,03)$ zwischen Säuglingen mit Koliken und nächtlicher Aufwachhäufigkeit im 12. Lebensmonat dar. Trotz des in Kapitel 5.2.5.1 erwähnten zahlenmäßigen Rückgangs der Säuglinge mit Koliken übersteigt die Zahl der Kinder mit weiterhin bestehenden Koliken und häufigen Aufwachepisoden pro Nacht die erwartete Häufigkeit um fast das Doppelte (beob. Häufigkeit: 9 (60 \%) vs. erw.: Häufigkeit: 5,3 (35,4 \%)). Das heißt: Säuglinge, die im 4. Lebensmonat noch deutliche Koliken zeigen wachen im Alter von 12 Monaten signifikant häufiger pro Nacht auf als Säuglinge ohne Koliken. In Abbildung 14 wird das Ergebnis dargestellt.

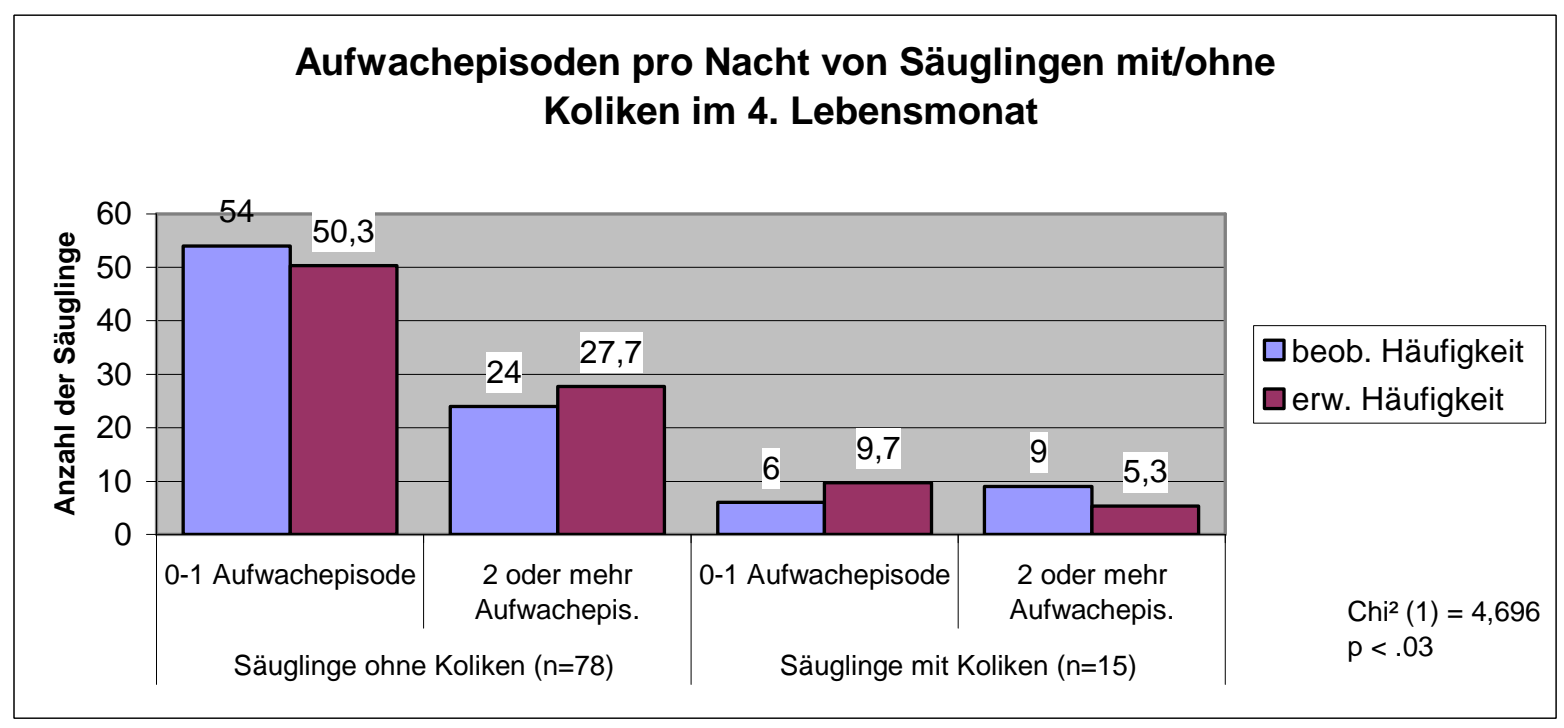

Abbildung 14: Aufwachepisoden pro Nacht im 12. Lebensmonat von Säuglingen mit/ohne Koliken im 4. Lebensmonat

Zusammenfassend kann das Auftreten von Koliken mit gehäuftem Aufwachen zum Ende des ersten Lebensjahres assoziiert werden. Dies wird in den ersten Lebensmonaten (Geburt bis 4. Lebensmonat) besonders bei der Betrachtung der Anzahl der Nächte mit Wachepisoden während der gesamten Woche deutlich. So zeigen Säuglinge, welche von der Geburt bis zum 4. Lebensmonat deutliche Koliken haben, im 
Alter von 12 Monaten eine höhere Anzahl von Nächten mit Wachepisoden pro Woche als Säuglinge mit wenigen Koliken. Hat der Säugling im Alter von 4 Monaten häufig Koliken, so äußert sich dies im Alter von 12 Monaten in einer verstärkten nächtlichen Aufwachfrequenz (siehe Abbildung 13 und 14). 


\subsection{Hypothesenprüfung}

Im folgenden Abschnitt steht die Untersuchung der Hauptmerkmale „Temperament des Säuglings“ sowie „Depressionsneigung und Ängstlichkeit der Bezugsperson“ zum Zeitpunkt des 4., 8. und 12. Lebensmonats im Vordergrund.

\subsubsection{Temperamentsmerkmale und Schlafverhalten}

\subsubsection{Temperamentsmerkmale}

Die Temperamentsmerkmale wurden, wie schon beschrieben, zum einen aus Elternfragebögen, zum anderen aus Reaktionen auf standardisierte Reize (Verhaltenstests) erfasst. Um eine möglichst objektive Darstellung der Temperamentsmerkmale zu erhalten, wird zunächst auf die Abhängigkeit bzw. Unabhängigkeit der Variablen eingegangen. Die Temperamentsmerkmale setzen sich aus den zwei Dimensionen „positive Emotionalität“ und „negative Emotionalität“ zusammen.

\subsection{Untersuchung der Temperamentsmerkmale bezüglich ihrer Inter- korrelation}

Die Interkorrelation wurde während aller drei Untersuchungszeiträume (4, 8 und 12 Monate) geprüft.

Tabelle 5 zeigt deutlich, dass sich die Variablen „positive und negative Emotion“ im Alter von 4 Monaten sowohl aus dem Elternurteil als auch aus den Verhaltenstests als weitgehend unabhängig darstellen. Es kann keine signifikante Korrelation nachgewiesen werden (vgl. Tabelle 5). 
Tabelle 5: Interkorrelation der Temperamentsmerkmale positive Emotionalität und negative Emotionalität im Alter der Säuglinge von 4 Monaten

\begin{tabular}{|c|l|l|}
\hline & Positive Emotionalität & \multicolumn{1}{|c|}{$\begin{array}{c}\text { Negative Emotionalität/ } \\
\text { Irritierbarkeit }\end{array}$} \\
\hline Positive Emotionalität & & Verhaltenstest: \\
$-0,11$ \\
\cline { 2 - 3 } $\begin{array}{c}\text { Negative Emotionalität / } \\
\text { Irritierbarkeit }\end{array}$ & $\begin{array}{l}\text { Elternurteil: } \\
-0,17^{\mathrm{t}}\end{array}$ & \\
\hline
\end{tabular}

Anmerkung: Signifikanzniveau: $\mathrm{t}: \mathrm{p}<0,10$

Mitgeteilt sind Pearson-Produkt-Moment-.Korrelationskoeffizienten.

Bei der Betrachtung der 8-monatigen Säuglinge zeigt sich ebenfalls die fast ausnahmslose Unabhängigkeit zwischen den positiven und negativen Emotionalitätsmaßen. Es ist nur eine geringe negative Korrelation feststellbar. Lediglich beim Signifikanzniveau von 5\% wird eine statistisch bedeutsame Korrelation zwischen „negativer Emotionalität//rritierbarkeit“ und „positiver Emotionalität“ im Elternurteil sichtbar, wobei sich die gemeinsame Varianz nur auf $4 \%$ beläuft. Grundsätzlich ist jedoch die Tendenz der Unabhängigkeit der zwei Dimensionen sowohl in den Elternurteilen als auch in den Verhaltenstests angezeigt (vgl. Tabelle 6).

Tabelle 6: Interkorrelation der Temperamentsmerkmale positive Emotionalität und negative Emotionalität im Alter der Säuglinge von 8 Monaten

\begin{tabular}{|c|l|l|}
\hline & \multicolumn{1}{|c|}{ Positive Emotionalität } & \multicolumn{1}{c|}{$\begin{array}{c}\text { Negative Emotionalität/ } \\
\text { Irritierbarkeit }\end{array}$} \\
\hline Positive Emotionalität & & $\begin{array}{l}\text { Verhaltenstest: } \\
-0,13\end{array}$ \\
\cline { 2 - 3 } $\begin{array}{c}\text { Negative Emotionalität / } \\
\text { Irritierbarkeit }\end{array}$ & $\begin{array}{l}\text { Elternurteil: } \\
-0,20^{*}\end{array}$ & \\
\hline
\end{tabular}

Anmerkung: Signifikanzniveau: ${ }^{*}: p<0,05$

Mitgeteilt sind Pearson-Produkt-Moment-.Korrelationskoeffizienten.

Im Alter von 12 Monaten bestätigen sich wiederum die schon zu den vorangegangenen Monaten erfassten Ergebnisse, welche eine Unabhängigkeit der beiden Dimensionen besonders im Verhaltenstest nachweisen. Einzig und allein lässt sich, wie 
schon bei der Interkorrelationsbeschreibung nach 8 Monaten angedeutet, eine jetzt noch deutlichere negative Korrelation der „negativen Emotionalität/Irritierbarkeit“ mit der „positiven Emotionalität“ im Elternurteil verzeichnen. Dies bedeutet, dass ein Säugling, der von seiner Bezugsperson häufig mit positivem Affektausdruck beschrieben wird dementsprechend auch selten negativ in seinen Reaktionen beschrieben wird (vgl. Tabelle 7).

Tabelle 7: Interkorrelation der Temperamentsmerkmale positive Emotionalität und negative Emotionalität im Alter der Säuglinge von 12 Monaten

\begin{tabular}{|c|l|l|}
\hline & \multicolumn{1}{|c|}{ Positive Emotionalität } & \multicolumn{1}{c|}{$\begin{array}{c}\text { Negative Emotionalität/ } \\
\text { Irritierbarkeit }\end{array}$} \\
\hline Positive Emotionalität & & Verhaltenstest: \\
& & 0,00 \\
\hline $\begin{array}{c}\text { Negative Emotionalität / } \\
\text { Irritierbarkeit }\end{array}$ & $\begin{array}{l}\text { Elternurteil: } \\
-0,38^{* * *}\end{array}$ & \\
\hline Anmerkung: Signifikanzniveau: $* * *: p<0,001$ &
\end{tabular}

Anmerkung: Signifikanzniveau: ${ }^{* \star *}: p<0,001$

Mitgeteilt sind Pearson-Produkt-Moment-.Korrelationskoeffizienten.

Abschließend kann die auf der Grundlage der Verhaltenstests basierende Unabhängigkeit der zwei Emotionalitätsmerkmale bestätigt werden.

\subsubsection{Korrelation zwischen Temperamentsmerkmalen und Schlaf-/ Wachperioden}

In diesem Abschnitt sollen nun die Zusammenhänge der Wachepisoden während der Nacht und die Häufigkeit der Nächte mit Schlafproblemen unter der Woche im 12. Lebensmonat mit den Temperamentsmerkmalen des Säuglings zu den drei Erhebungszeitpunkten untersucht werden. 


\subsection{Zusammenhänge zwischen positiver Emotionalität und Schlafver- halten}

Die positive Emotionalität des Kindes stellt nach Laucht et al. (1997) kindliche Ressourcen dar. In Anbetracht dieser These sollen nun die Zusammenhänge zwischen positiver Emotionalität aus den Verhaltenstests sowie aus der Skala „Lachen/ Lächeln“ des „Infant Behavior Questionaire“ (IBQ) mit dem Schlafverhalten der Säuglinge dargestellt werden.

Aus den gewonnenen Ergebnissen geht deutlich hervor: Je höher die positive Emotionalität des Säuglings im Eltern-Fragebogen und im Verhaltenstest ausgeprägt ist, desto seltener wird der Nachtschlaf im 12. Lebensmonat durch Wachepisoden unterbrochen.

Mit besonderer Deutlichkeit zeigt sich dies bei den Elternurteilen (Skala „Lachen/ Lächeln“) mit den Wachepisoden pro Nacht. Im Alter von 4 und 12 Monaten liegt hier das Signifikanzniveau bei $<1 \%$ und die Korrelationen betragen jeweils $r=-0,299$ (4 Monaten) und $r=-0,287$ (12 Monaten).

Im Alter von 8 Monaten zeigt sich lediglich ein Ergebnis mit einem tendenziellen Charakter $(p<0,059)$ (siehe Tabelle 8$)$.

Eine Bestätigung der oben genannten Ergebnisse wurde durch die Auswertung der Verhaltenstests erreicht. Hierbei zeigt sich besonders im Alter von 8 und 12 Monaten ein deutlicher Zusammenhang zwischen positiver Emotionalität und nächtlichem Durchschlafen im 12. Lebensmonat. Das Signifikanzniveau beträgt $<5 \%$ bei einer Korrelation von $r=-0,232$ (8 Monaten) und $r=-0,221$ (12 Monaten).

Im Alter von 4 Monaten kann kein signifikantes Ergebnis festgestellt werden. Eine Übersicht erfolgt in Tabelle 8. 
Tabelle 8: Pearson Rangkorrelationskoeffizienten der positiven Emotionalität mit den nächtlichen Aufwachepisoden im 12. Lebensmonat

\begin{tabular}{|c|c|c|c|c|c|c|}
\hline & & \multicolumn{5}{|c|}{ Aufwachepisoden pro Nacht } \\
\hline & & 4 Monate & & 8 Monate & & 12 Monate \\
\hline Fragebogenskala & & $r=-0,299$ & & $r=-0,201$ & & $r=-0,287$ \\
\hline „Lachen / Lä- & & $p<0,004$ & & $p<0,059$ & & $p<0,006$ \\
\hline cheln“" & & $\mathbf{n}=93$ & * & $\mathbf{n}=89$ & * & $\mathrm{n}=92$ \\
\hline Verhaltenstest & & $r=-0,063$ & & $r=-0,232$ & & $r=-0,221$ \\
\hline „,positive Emotio- & & ns & & $p<0,029$ & & $p<0,033$ \\
\hline nalität‘، & & $\mathrm{n}=88$ & $\star *$ & $\mathbf{n}=89$ & ** & $n=93$ \\
\hline
\end{tabular}

Anmerkung: *Mitgeteilt sind Phi-Korrelationskoeffizienten und Irrtumswahrscheinlichkeit (p); ns: nicht statistisch signifikant. ${ }^{* *}$ Mitgeteilt sind punktbiseriale Korrelationskoeffizienten und Irrtumswahrscheinlichkeit (p); ns: nicht statistisch signifikant.

Die oben genannten Ergebnisse zum Untersuchungszeitpunkt des 12. Lebensmonats werden mit Hilfe von Abbildung 15 verdeutlicht. Hier ist klar zu erkennen, dass Säuglinge mit deutlich positiver Emotionalität im Vergleich zu Säuglingen mit geringerer positiver Emotionalität nachts besser Durchschlafen.

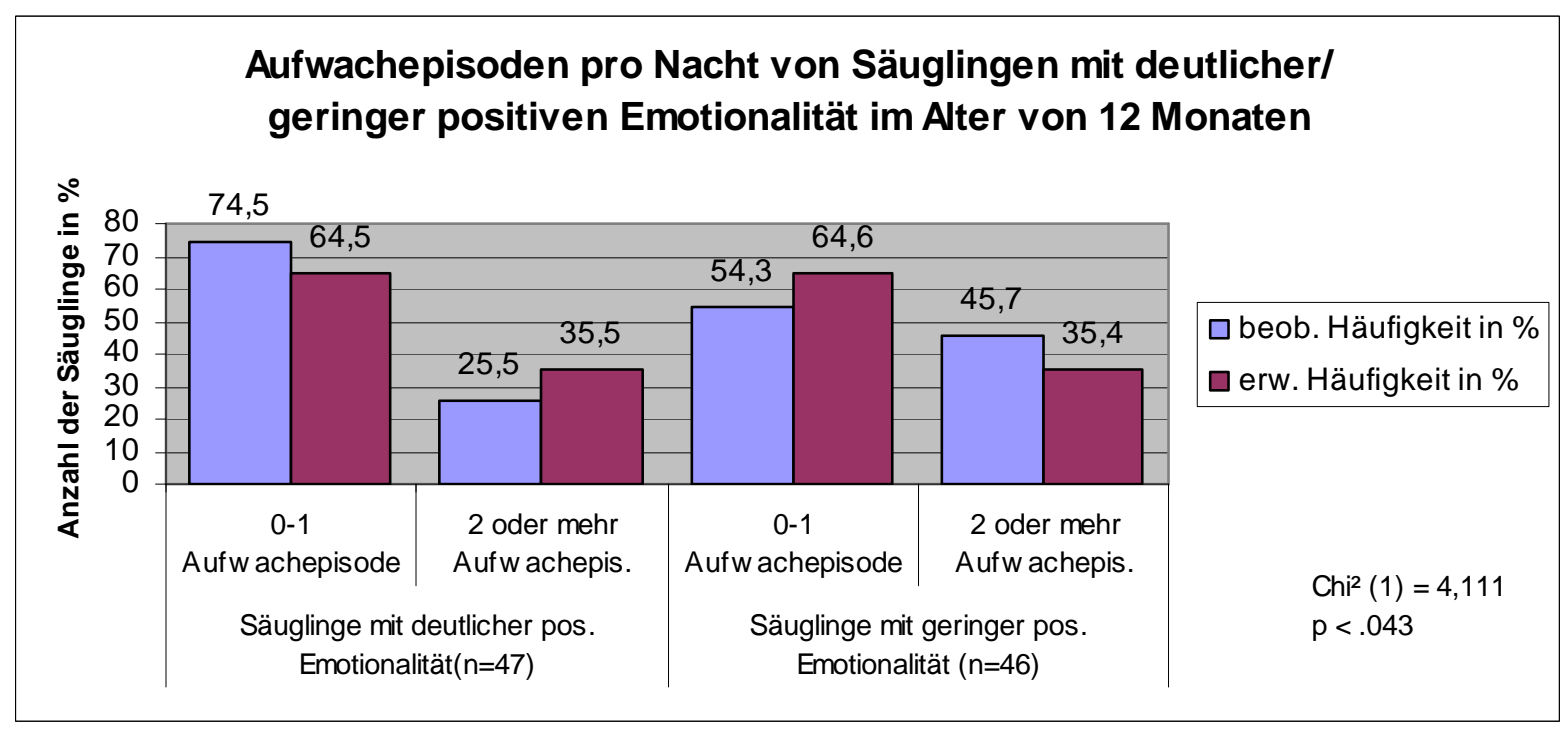

Abbildung 15: Aufwachepisoden pro Nacht von Säuglingen mit deutlicher/ geringer positiver Emotionalität im Alter von 12 Monaten 
Bei der Untersuchung des Zusammenhangs von wöchentlicher Aufwachhäufigkeit im 12. Lebensmonat mit der positiven Emotionalität des Säuglings kann zu keinem Untersuchungszeitpunkt weder im Elternurteil noch im Verhaltenstest ein signifikanter Zusammenhang festgestellt werden (siehe Tabelle 9).

Tabelle 9: Pearson Rangkorrelationskoeffizienten der positiven Emotionalität mit den Nächten, in denen Aufwachepisoden über die Woche betrachtet auftraten

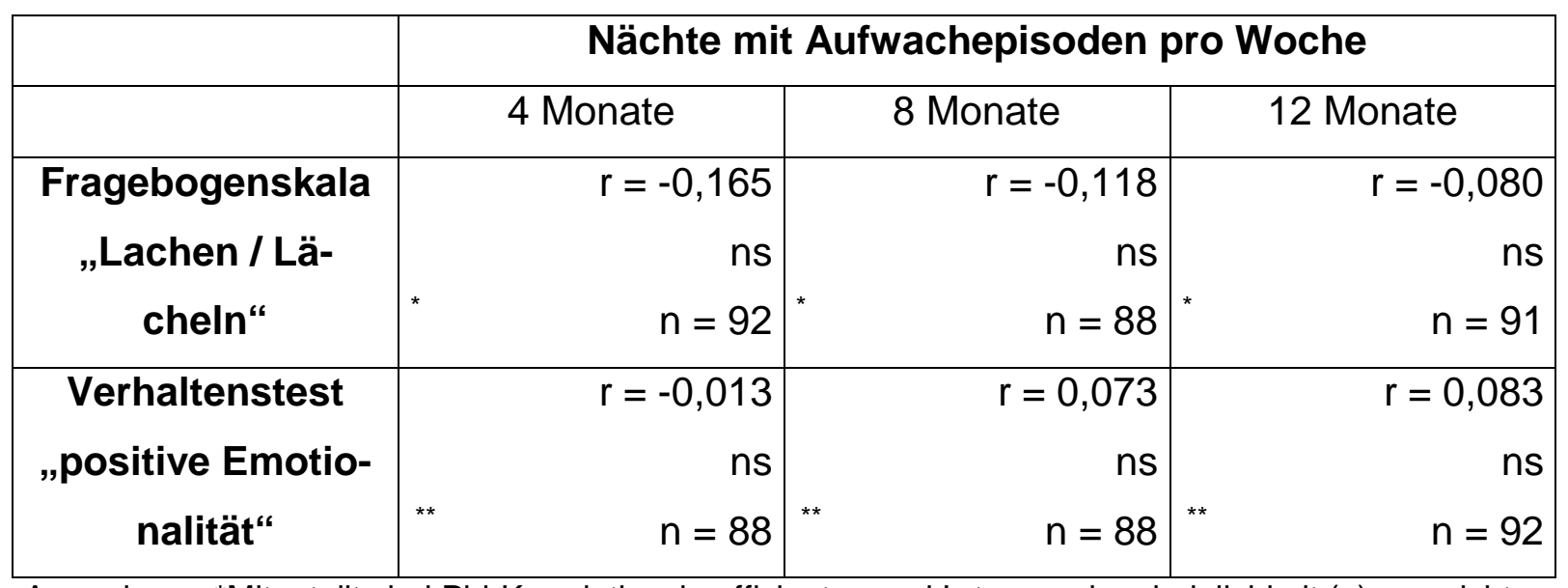

Anmerkung: *Mitgeteilt sind Phi-Korrelationskoeffizienten und Irrtumswahrscheinlichkeit (p); ns: nicht statistisch signifikant. ${ }^{* *}$ Mitgeteilt sind punktbiseriale Korrelationskoeffizienten und Irrtumswahrscheinlichkeit (p); ns: nicht statistisch signifikant.

Zusammenfassend kann das Vorhandensein positiver Emotionalität als förderlich in Bezug auf die nächtliche Durchschlafwahrscheinlichkeit im 12. Lebensmonat des Säuglings angesehen werden.

\subsection{Zusammenhänge zwischen negativer Emotionalität und Schlafver- halten}

An dieser Stelle soll der Frage nachgegangen werden, ob sich eine vermehrte Irritierbarkeit und ein damit verbunden erhöhtes Auftreten negativer Emotionalität im nächtlichen Schlafverhalten im 12. Lebensmonat der Säuglinge wiederspiegelt. Dies geschieht anhand Berechnung der Korrelationen negativer Temperamentsmerkmale mit den nächtlichen und wöchentlichen Aufwachepisoden. Die hierfür benötigten Daten wurden analog zur Erfassung der positiven Emotionalität durch Verhaltenstests und Elternfragebögen in Form der deutschsprachigen Adaptation des „Infant Behavi- 
or Questionaire“ (IBQ) von Pauli-Pott et al. (2003) mit der Skala „Unbehagen bei Frustration/ Einschränkungen“ im Alter von 4, 8 und 12 Monaten erfasst.

Ein über den gesamten Untersuchungszeitraum (4, 8 und 12 Monate) signifikantes Ergebnis ist bei Säuglingen, welche von ihrer Bezugsperson mittels der Skala „Unbehagen bei Frustration/Einschränkung“ als schnell frustriert und unzufrieden beschrieben werden, nicht zu erkennen.

Lediglich in der Anzahl der wöchentlichen Aufwachhäufigkeit im 12. Monat bei Säuglingen mit geringer Frustrationstoleranz im Alter von 4 Monaten lässt sich eine signifikante Korrelation erkennen $(r=0,220, p<0,036)$. Das heißt: Ein Säugling, der mit 4 Monaten als schnell frustriert und unzufrieden beschrieben wird, wacht im Alter von 12 Monaten pro Woche häufiger auf als ein zufriedener, ausgeglichener Säugling.

Ansonsten zeigt sich zu den übrigen Untersuchungszeitpunkten bei der wöchentlichen Aufwachhäufigkeit kein signifikanter Zusammenhang zum Fragebogenergebnis der Eltern oder bei der Untersuchung mittels Verhaltenstest (siehe Tabelle 10).

Tabelle 10: Pearson Rangkorrelationskoeffizienten der negativen Emotionalität mit den Nächten, in denen Aufwachepisoden über die Woche betrachtet auftraten

\begin{tabular}{|c|c|c|c|}
\hline & \multicolumn{3}{|c|}{ Nächte mit Aufwachepisoden pro Woche } \\
\hline & 4 Monate & 8 Monate & 12 Monate \\
\hline $\begin{array}{c}\text { Fragebogenskala } \\
\text { „Unbehagen bei } \\
\text { Frustration/ Ein- } \\
\text { schränkung“ }\end{array}$ & $\begin{array}{r}r=0,220 \\
p<0,036 \\
n=91\end{array}$ & $\begin{array}{r}r=0,144 \\
n s \\
n=89\end{array}$ & $\begin{array}{r}r=0,156 \\
n s \\
n=91\end{array}$ \\
\hline $\begin{array}{c}\text { Verhaltenstest } \\
\text { „negative Emotio- } \\
\text { nalität“ }\end{array}$ & $\begin{array}{r}r=-0,099 \\
n s \\
n=85\end{array}$ & $\begin{array}{r}r=0,049 \\
n s \\
n=88\end{array}$ & $\begin{array}{r}r=0,001 \\
n s \\
n=92\end{array}$ \\
\hline
\end{tabular}

Anmerkung: *Mitgeteilt sind Phi-Korrelationskoeffizienten und Irrtumswahrscheinlichkeit (p); ns: nicht statistisch signifikant. ${ }^{* *}$ Mitgeteilt sind punktbiseriale Korrelationskoeffizienten und Irrtumswahrscheinlichkeit (p); ns: nicht statistisch signifikant.

Bei der Betrachtung der Verhaltenstests in Bezug auf die nächtliche Aufwachhäufigkeit im 12. Lebensmonat stellt sich zum Untersuchungszeitpunkt des 12. Monats eine deutlich statistische Verbindung heraus $(r=0,259$; $p<0,012)$. Folglich weisen Säuglinge mit erhöhter Frustrationsneigung und schnellem Unbehagen bei Einschränkung 
im Alter von einem Jahr eine höhere Aufwachhäufigkeit pro Nacht auf als gering negativ emotionell veranlagte Säuglinge.

Ansonsten zeigt sich auch hier zu den übrigen Untersuchungszeitpunkten bei der nächtlichen Aufwachhäufigkeit kein weiterer signifikanter Zusammenhang mit den Untersuchungen mittels Verhaltenstest oder zum Fragebogenergebnis der Eltern (siehe Tabelle 11).

Tabelle 11: Pearson Rangkorrelationskoeffizienten der negativen Emotionalität mit den nächtlichen Aufwachepisoden

\begin{tabular}{|c|c|c|c|}
\hline & \multicolumn{3}{|c|}{ Aufwachepisoden pro Nacht } \\
\hline & 4 Monate & 8 Monate & 12 Monate \\
\hline $\begin{array}{c}\text { Fragebogenskala } \\
\text { „Unbehagen bei } \\
\text { Frustration/ Ein- } \\
\text { schränkung }\end{array}$ & $\begin{array}{r}r=0,079 \\
n s \\
n=92\end{array}$ & $\begin{array}{r}r=0,069 \\
n s \\
n=90\end{array}$ & $\begin{array}{r}r=0,155 \\
n s \\
n=92\end{array}$ \\
\hline $\begin{array}{c}\text { Verhaltenstest } \\
\text { „negative Emotio- } \\
\text { nalität“ }\end{array}$ & $\begin{array}{r}r=-0,047 \\
n s \\
n=85\end{array}$ & $\begin{array}{r}r=0,131 \\
n s \\
n=89\end{array}$ & $\begin{array}{r}r=0,259 \\
p<.012 \\
n=93\end{array}$ \\
\hline
\end{tabular}

Anmerkung: *Mitgeteilt sind Phi-Korrelationskoeffizienten und Irrtumswahrscheinlichkeit (p); ns: nicht statistisch signifikant. ${ }^{* *}$ Mitgeteilt sind punktbiseriale Korrelationskoeffizienten und

Irrtumswahrscheinlichkeit (p); ns: nicht statistisch signifikant.

Die oben genannten Ergebnisse zum Untersuchungszeitpunkt des 12. Lebensmonats werden mit Hilfe von Abbildung 16 verdeutlicht. Hier ist klar zu erkennen, dass $43(74,1 \%)$ der insgesamt 58 einjährigen Säuglinge mit geringer negativer Temperamentsbeurteilung aus dem Verhaltenstest keine bzw. nur eine geringe Anzahl an nächtlichen Aufwachepisoden (0-1) am Ende des ersten Lebensjahres zu verzeichnen haben. Demgegenüber weisen 18 (51,4\%) der insgesamt 35 Säuglinge gleichen Alters mit deutlicher negativer Emotionalität zwei oder mehr nächtliche Wachepisoden am Ende des ersten Lebensjahres auf $\left(\operatorname{Chi}^{2}(1)=6,232 ; p<0,013\right)$.

Zum Befragungszeitpunkt im 4. und 8. Monat stellen sich die Ergebnisse der Verbindung von Verhaltenstest und nächtlicher/wöchentlicher Aufwachhäufigkeit im Alter von 12 Monaten statistisch als nicht signifikant dar. 


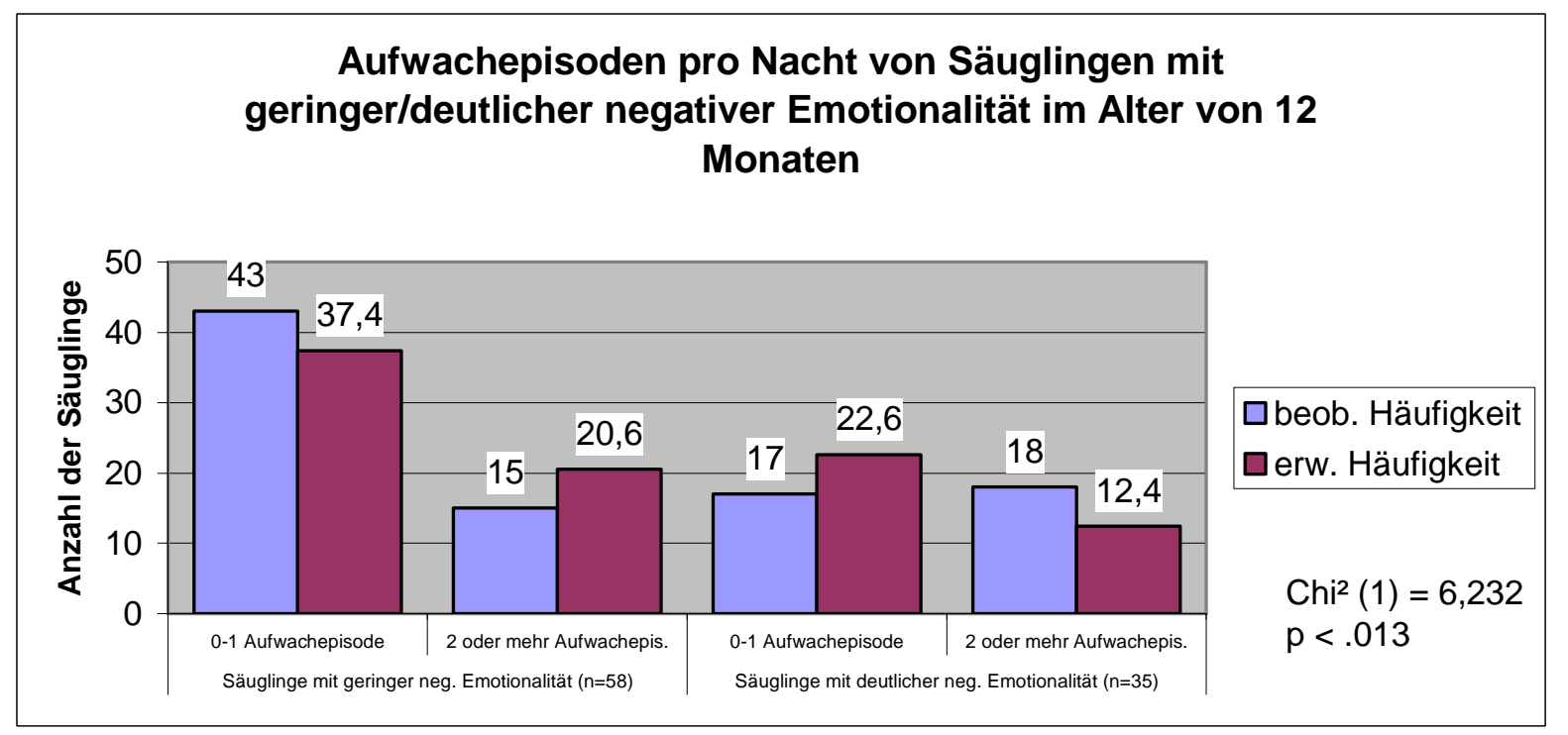

Abbildung 16: Aufwachepisoden pro Nacht im 12. Lebensmonat von Säuglingen mit geringer/ deutlicher negativer Emotionalität im Alter von 12 Monaten 


\subsubsection{Mütterliche Merkmale und kindliches Schlafverhalten}

Die Erfassung der Depressionsneigung und der Ängstlichkeit der Bezugsperson wurde zu allen drei Untersuchungszeitpunkten (4., 8., 12. Monat) mit Hilfe der Fragebogenskalen „Depressivität“ und der Skala „Überfürsorge aus Angst“ aus dem „Fragebogen zur Erfassung der Erziehungseinstellungen von Müttern mit Kindern im Kleinstkindalter“ (EMKK) von Engfer (1984), der „Skala Hoffnungslosigkeit“ von Beck in der deutschen Bearbeitung von Krampen (1979) und der Skala „Trait-Angst“ aus dem „State-Trait-Angstinventar“ (STAI) von Laux, Glanzmann, Schaffer und Spielberger (1981) durchgeführt.

\subsubsection{Korrelation der mütterlichen Merkmale mit den Aufwachepisoden des Säuglings}

Im Folgenden werden die Zusammenhänge der mütterlichen Depressionsneigung und Ängstlichkeit während der Untersuchungszeitpunkte 4., 8. und 12. Monat mit dem Schlafverhalten des Kindes im 12. Lebensmonat untersucht.

Bei der Betrachtung von Müttern, welche sich in der Fragebogenskala „Depressivität“ als wenig depressiv und unsicher beschreiben, zeigt sich eine deutlich positive Korrelation zum Untersuchungszeitpunkt im 4. $(r=0,231 ; p=0,029)$ und 12. Monat $(r=$ 0,223; $p=0,046)$ mit der nächtlichen Aufwachhäufigkeit des Kindes im Alter von 12 Monaten. Hieraus lässt sich ablesen, dass Kinder mit selbstbewussten und wenig depressiven Müttern im Alter von 12 Monaten nachts seltener aufwachen. Zum Untersuchungszeitpunkt im 8. Monat lässt sich das vorangegangene Ergebnis nicht signifikant darstellen (siehe Tabelle 12). 
Tabelle 12: Pearson Rangkorrelationskoeffizienten der Skalen „Depressivität“ im 4., 8. und 12. Monat mit den nächtlichen Aufwachepisoden der Säuglinge im Alter von 12 Monaten

\begin{tabular}{|c|r|r|r|}
\hline & \multicolumn{3}{|c|}{ Aufwachepisoden pro Nacht } \\
\hline & 4 Monate & 8 Monate & \multicolumn{1}{|c|}{12 Monate } \\
\hline Fragebogenskala & $\mathbf{r}=\mathbf{0 , 2 3 1}$ & $\mathrm{r}=0,139$ & $\mathbf{r = 0 , 2 2 3}$ \\
"Depressivität“ & $\mathbf{p}<. \mathbf{0 2 9}$ & $\mathrm{ns}$ & $\mathbf{p}<. \mathbf{0 4 6}$ \\
\hline
\end{tabular}

Anmerkung: Mitgeteilt sind punktbiseriale Korrelationskoeffizienten und Irrtumswahrscheinlichkeit (p); ns: nicht statistisch signifikant.

Ähnliche Ergebnisse zeigt die statistisch signifikante Korrelation der Skala „Hoffnungslosigkeit“ der Bezugsperson zum Untersuchungszeitpunkt im 4. Lebensmonat mit den Schlafgewohnheiten des Kindes im 12. Lebensmonat $(r=0,209 ; p=0,044)$. Das bedeutet: Säuglinge von Müttern, welche sich zum Zeitpunkt des 4. Lebensmonats mit negativen Denkstrukturen über ihre eigene Person, die Umwelt und die $\mathrm{Zu}$ kunft beschreiben, weisen mit 12 Monaten eine deutlich höhere Aufwachhäufigkeit pro Nacht auf als Säuglinge von Müttern mit positiven Denkstrukturen.

Zum Untersuchungszeitpunkt im 8. und 12. Monat lässt sich kein signifikantes Ergebnis darstellen (siehe Tabelle 13).

Tabelle 13: Pearson Rangkorrelationskoeffizienten der Fragebogenskala „Hoffnungslosigkeit“ im 4., 8. und 12. Monat mit den nächtlichen Aufwachepisoden der Säuglinge im Alter von 12 Monaten

\begin{tabular}{|c|r|r|r|}
\hline & \multicolumn{3}{|c|}{ Aufwachepisoden pro Nacht } \\
\hline & 4 Monate & 8 Monate & 12 Monate \\
\hline $\begin{array}{c}\text { Fragebogenskala } \\
\text { „Hoffnungslosig- } \\
\text { keit“ }\end{array}$ & $\mathbf{r}=\mathbf{0 , 2 0 9}$ & $\mathrm{r}=0,040$ & $\mathrm{r}=0,099$ \\
$\mathrm{p}<.044$ & $\mathrm{~ns}$ \\
\hline
\end{tabular}

Anmerkung: Mitgeteilt sind punktbiseriale Korrelationskoeffizienten und Irrtumswahrscheinlichkeit (p); ns: nicht statistisch signifikant.

Erstaunlicherweise lässt sich eine signifikante Korrelation bei ängstlichen Müttern im Hinblick auf die nächtliche Aufwachhäufigkeit ihres Kindes am Ende des ersten Lebensjahres zu keinem Untersuchungszeitpunkt (4., 8., 12. Monat) zeigen. Die Skala „Überfürsorge aus Angst“ korreliert jedoch im 4. und 8. Monat tendenziell mit der 
Häufigkeit des Aufwachens des Säuglings im Alter von 12 Monaten. Dies beinhaltet: Wenn die Mutter sorglos und angstfrei mit dem Kind interagiert, zeigt sich eine tendenziell geringere Aufwachhäufigkeit des Säuglings pro Nacht im 12. Lebensmonat. Zum Untersuchungszeitpunkt im 12. Monat lässt sich kein signifikantes Ergebnis darstellen (siehe Tabelle 14).

Tabelle 14: Pearson Rangkorrelationskoeffizienten der Fragebogenskala „Trait-Angst“ und „Überfürsorge aus Angst“ im 4., 8. und 12. Monat mit den nächtlichen Aufwachepisoden der Säuglinge im Alter von 12 Monaten

\begin{tabular}{|c|r|r|r|}
\hline & \multicolumn{3}{|c|}{ Aufwachepisoden pro Nacht } \\
\hline & 4 Monate & 8 Monate & 12 Monate \\
\hline $\begin{array}{c}\text { Fragebogenskala } \\
\text { „Trait- Angst“ }\end{array}$ & $r=0,132$ & $r=0,095$ & $r=0,067$ \\
\hline $\begin{array}{c}\text { Fragebogenskala } \\
\text { Überfürsorge aus }\end{array}$ & $\mathbf{r}=\mathbf{0 , 2 0 0}$ & $\mathbf{n s}$ & $\mathrm{ns}$ \\
Angst“ & $\mathbf{p}<\mathbf{0 , 0 5 7}$ & $\mathbf{p}<0,076$ & $\mathrm{~ns}$ \\
\hline
\end{tabular}

Anmerkung: Mitgeteilt sind punktbiseriale Korrelationskoeffizienten und Irrtumswahrscheinlichkeit (p); ns: nicht statistisch signifikant.

Abschließend wurde die Summe über allen Depressivitäts- und Angstskalen während der drei Untersuchungszeitpunkte (4., 8. und 12. Monat) gebildet und auf mögliche Korrelationen zur Aufwachhäufigkeit des Kindes im Alter von 12 Monaten untersucht. So zeigte sich zum Untersuchungszeitpunkt 4. Monat bei Müttern, welche vermehrt Überforderung und Bedrücktheit in der Beziehung zu ihrem Kind angeben und deren Mutterrolle von Selbstzweifeln geprägt ist, eine statistisch signifikante Korrelation ( $r=$ $0,234, p<0,025)$ zur Aufwachhäufigkeit ihres Kindes pro Nacht im 12. Lebensmonat. Das besagt: Je unsicherer und gehemmter sich die Mutter im 4. Lebensmonat beschreibt, um so häufiger wacht der Säugling am Ende des ersten Jahres pro Nacht auf.

Im Alter von 8 und 12 Monaten zeigt sich hingegen kein signifikantes Ergebnis (siehe Tabelle 15). 
Tabelle 15: Pearson Rangkorrelationskoeffizienten der $\Sigma$ über allen Depressivitäts- und Angstskalen im 4., 8. und 12. Monat mit den nächtlichen Aufwachepisoden der Säuglinge im Alter von 12 Monaten

\begin{tabular}{|c|r|r|r|}
\hline & \multicolumn{3}{|c|}{ Aufwachepisoden pro Nacht } \\
\hline & 4 Monate & 8 Monate & 12 Monate \\
\hline $\begin{array}{c}\text { über allen De- } \\
\text { pressivitäts- und } \\
\text { Angstskalen }\end{array}$ & $\mathbf{r}=\mathbf{0 , 2 3 4}$ & $r=0,110$ & $\mathrm{r}=0,124$ \\
$\mathrm{~ns}$ & $\mathrm{~ns}$ \\
\hline
\end{tabular}

Anmerkung: Mitgeteilt sind punktbiseriale Korrelationskoeffizienten und Irrtumswahrscheinlichkeit (p); ns: nicht statistisch signifikant.

Die wöchentliche Aufwachfrequenz im Alter von 12 Monaten zeigt zu keinem Erhebungszeitpunkt eine statistisch signifikante Korrelation mit oben genannten Fragebogenskalen zur Depressionsneigung und Ängstlichkeit der Bezugsperson. 


\subsection{Multiple Korrelation und Regression}

Im folgenden Kapitel soll das Augenmerk auf mögliche Beziehungen zwischen den für die Untersuchung genutzten Prädiktorvariablen und den beiden Kriteriumsvariablen „Anzahl der Aufwachepisoden pro Nacht“ und „Anzahl der Nächte mit Wachepisoden pro Woche" gerichtet sein. Ziel hierbei ist, die Prädiktorvariablen ausfindig zu machen, welche einen signifikanten Beitrag zur Vorhersage der einzelnen Kriteriumsvariablen, in unserem Fall der Aufwachfrequenz der Säuglinge pro Nacht und pro Woche über die anderen Variablen hinaus leisten. Dabei soll geprüft werden, ob die Merkmale Depressivität/Ängstlichkeit der Mutter und die Temperamentsmerkmale voneinander unabhängig und über die Kontrollmerkmale hinaus mit dem Schlafverhalten im Alter von 12 Monaten korrelieren. Hierzu werden multiple Regressionsanalysen mit schrittweiser Variablenauswahl verwendet. Bei diesen multivariaten Analysen sollen alle bivariat mit den Schlafmerkmalen signifikant verbundenen Variablen berücksichtigt werden. Aus diesem Satz der signifikanten Prädiktoren wählt das schrittweise Regressionsverfahren jene Prädiktoren aus, die am engsten und unabhängig von den übrigen Prädiktoren mit dem Schlafmerkmal korrelieren.

Tabelle 16 enthält eine Zusammenstellung all jener Merkmale, die mit den beiden Schlafverhaltensmerkmalen „Anzahl der Wachepisoden pro Nacht“ und „Anzahl der Nächte mit Wachepisoden pro Woche" signifikant verbunden waren. Kinder, die im Alter von 12 Monaten durchschlafen, wurden von ihren Müttern im Alter von 4, 8 und 12 Monaten als häufig lächelnd und lachend beschrieben. Sie zeigten in den Verhaltenstests im Alter von 8 und 12 Monaten einen mehr positiven Emotionsausdruck und im Alter von 12 Monaten seltener einen negativen Emotionsausdruck. Die Mütter dieser Kinder beschrieben sich im Alter der Kinder von 4 und 12 Monaten als weniger depressiv, im Alter von 4 und 8 Monaten als weniger ängstlich sowie im Alter von 4 Monaten als weniger hoffnungslos.

Weiterhin zeigte sich bei Auswertung der Kontrollvariablen, dass Kinder, die mit 12 Monaten besser durchschlafen, vom 4. bis 8. Lebensmonat lediglich einmal bzw. keinmal den Arzt konsultierten (ausgenommen die Vorsorgeuntersuchungen). Ebenso zeigte sich bei diesen gut schlafenden Kindern zum Zeitpunkt der Geburt, der Vorsorgeuntersuchungen U3 (4. bis 6. Lebenswoche) und U6 (10. bis 12. Lebens- 
monat) ein höheres Körpergewicht als bei den häufig aufwachenden Säuglingen. Die Säuglinge mit wenigen Aufwachepisoden pro Nacht zum Zeitpunkt des 12. Lebensmonat wiesen im Alter von 4 Monaten ebenfalls weniger Koliken (Perioden unberuhigbaren Schreiens) auf.

Mit der Anzahl der Nächte pro Woche, in denen das Kind nachts aufwacht, ergab sich lediglich eine statistisch bedeutsame Korrelation mit der Skala „Unbehagen bei Frustration/Einschränkung“ im Alter der Säuglinge von 4 Monaten. Bei den Kontrollvariablen korrelierten die Häufigkeit der Arztbesuche im Zeitraum vom 4. bis 8. Monat, der kognitive Entwicklungsstand des Säuglings im Alter von 12 Monaten und die Häufigkeit von Koliken (Perioden unberuhigbaren Schreiens) von der Geburt bis zum 4. Lebensmonat signifikant. 
Tabelle 16: Übersicht der mit der Aufwachhäufigkeit pro Nacht und Woche signifikant verknüpften Merkmale

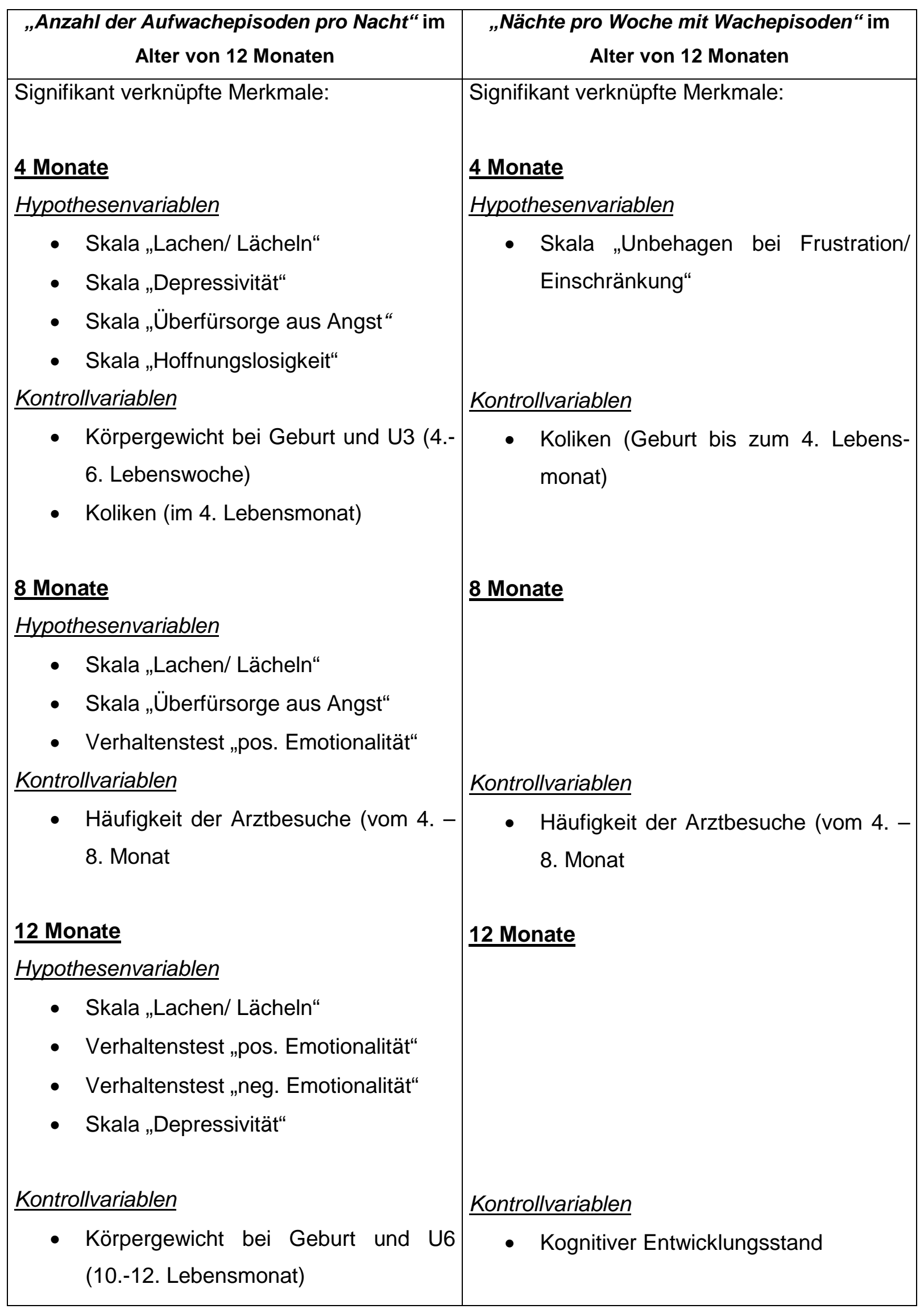


Im Rahmen der Regressionsanalysen werden die im Alter von 4 und 8 Monaten erhobenen Prädiktoren berücksichtigt.

Das Merkmal „Anzahl der Aufwachepisoden pro Nacht“ lässt sich am besten durch die Merkmale geringes Geburtsgewicht, Depression der Mutter im 4. Lebensmonat des Säuglings und geringe positive Emotionalität im Alter von 8 Monaten vorhersagen. Diese Merkmale korrelieren unabhängig voneinander und über die im Alter von 8 Monaten vorliegende Schlafdauer hinaus mit dem Kriterium (vgl. Tabelle 17).

Tabellen 17 a,b,c: Ergebnisse der Regressionsanalyse zum Merkmal „Anzahl der Aufwachepisoden pro Nacht“"

\begin{tabular}{|l|lll|}
\hline & \multicolumn{3}{|l|}{ Änderungsstatistiken } \\
& $\mathrm{R}^{2}$ & $\mathrm{~F}$ & $\mathrm{p}$ \\
\hline $\begin{array}{l}\text { 1. Skala Lä- } \\
\text { cheln/Lachen (4 Mon.) }\end{array}$ & .09 & 8.55 & .004 \\
\cline { 1 - 1 } $\begin{array}{l}\text { 2. Schlafdauer ohne } \\
\text { Unterbrechung } \\
\text { (8 Mon.) }\end{array}$ & .07 & 6.73 & .011 \\
\cline { 1 - 1 } 3. Geburtsgewicht & .05 & 5.45 & .022 \\
\cline { 1 - 1 } $\begin{array}{l}\text { 4. Depressivität } \\
\text { (4 Mon.) }\end{array}$ & .05 & 6.15 & 0.015 \\
\cline { 1 - 1 } $\begin{array}{l}\text { 5. Ausschluss: Skala } \\
\text { Lachen/ Lächeln } \\
\text { (4 Mon.) }\end{array}$ & - & - & - \\
\cline { 1 - 1 } $\begin{array}{l}\text { 6. pos. Emotionalität } \\
\text { (Verhaltenstest) } \\
\text { (8 Mon.) }\end{array}$ & .04 & 4.47 & .038 \\
\hline
\end{tabular}




\begin{tabular}{|c|c|c|c|}
\hline & \multicolumn{3}{|c|}{$\begin{array}{l}\text { Koeffizienten der Regres- } \\
\text { sionsgleichung (Gesamt- } \\
\text { gleichung) }\end{array}$} \\
\hline & Beta & $\mathrm{t}$ & $p$ \\
\hline $\begin{array}{l}\text { Schlafdauer ohne } \\
\text { Unterbrechung } \\
\text { (8 Mon.) }\end{array}$ & -.33 & 3.57 & .001 \\
\hline Geburtsgewicht & -.28 & 2.88 & .005 \\
\hline Depressivität & .28 & 3.05 & .003 \\
\hline Pos. Emotionalität & -.20 & 2.11 & .038 \\
\hline
\end{tabular}

\begin{tabular}{|lll|}
\hline \multicolumn{3}{|l|}{ Signifikanz der Gesamtgleichung } \\
$\mathrm{R}$ & $\mathrm{F}$ & $\mathrm{p}$ \\
\hline .53 & 8.24 & $<.001$ \\
\hline
\end{tabular}

Das Merkmal „Anzahl der Nächte pro Woche mit Wachepisoden“ war mit beschriebenen Säuglingskoliken, der Fragebogenskala „Unbehagen bei Frustration/Einschränkung“ und der Anzahl der Arztbesuche bivariat signifikant verbunden. In der schrittweisen Regressionsanalyse werden die Merkmale Säuglingskoliken und Arztbesuche als beste und voneinander unabhängige Prädiktoren identifiziert (vgl. Tabellen 18).

Tabellen 18 a,b: Ergebnisse der Regressionsanalyse zum Merkmal „Anzahl der Nächte pro Woche mit Wachepisoden“

\begin{tabular}{|c|c|c|c|c|c|c|}
\hline & \multicolumn{3}{|c|}{ Änderungsstatistiken } & \multicolumn{3}{|c|}{$\begin{array}{l}\text { Koeffizienten der } \\
\text { Regressionsgleichung } \\
\text { (Gesamtgleichung) }\end{array}$} \\
\hline & $\mathrm{R}^{2}$ & $\mathrm{~F}$ & $p$ & Beta & $\mathrm{t}$ & $\mathrm{p}$ \\
\hline Anzahl der Arztbesuche & .10 & 9,87 & .002 & .29 & 2.88 & .005 \\
\hline Koliken & .07 & 6,80 & .011 & .26 & 2.61 & .011 \\
\hline
\end{tabular}

\begin{tabular}{|lll|}
\hline \multicolumn{3}{|l|}{ Signifikanz der Gesamtgleichung } \\
$\mathrm{R}$ & $\mathrm{F}$ & $\mathrm{p}$ \\
\hline .41 & 8,67 & $<.001$ \\
\hline
\end{tabular}




\section{Diskussion}

\subsection{Das Schlafverhalten}

Das Schlafverhalten von Säuglingen ist besonders während des ersten Lebenshalbjahres nicht auf einen durchgängigen Nachtschlaf ausgerichtet, vielmehr verhält sich das häufige nächtliche Aufwachen adaptativ zur häufigen Nahrungsaufnahme und dem Wachstum in der Anfangszeit (Wolke et al., 1998; Skuse et al., 1994). Allerdings äußert sich das häufige Aufwachen nicht unumgänglich in einer Schlafproblematik. Lediglich beim kindlichen Unvermögen, selbständig in den Schlaf zurückzufinden, werden die Schlafprobleme offenbar (Minde et al., 1993; Gaylor et al., 1998).

\subsubsection{Schlaf-/Wachperioden der Stichprobe}

Die Säuglinge der Stichprobe zeigten im Alter von 8 Monaten zum größten Teil ein als regelmäßig zu bezeichnendes Schlafverhalten mit durchschnittlich zwei Tagschlafperioden. Die durchschnittliche Dauer der nächtlichen Schlafperiode ohne Unterbrechung betrug hier ungefähr 8 Stunden.

Da es aus technischen Gründen unmöglich war, alle Aufwachepisoden des Kindes, insbesonders jene, in denen es selbständig wieder in den Schlaf fand, videosomnographisch festzuhalten, konnten lediglich solche Wachepisoden erfasst werden, in denen der Säugling die Aufmerksamkeit der Eltern auf sich richtete. So zeigten sich bei knapp zwei Drittel $(61 \%)$ der Stichprobe $(n=93)$ nur vereinzelte bzw. keine Aufwachepisoden pro Nacht. Jedoch wiesen immerhin noch gut ein Drittel (34\%) der Säuglinge vermehrte nächtliche Aufwachepisoden (2 oder mehr) auf (Kriteriumsvariable: Anzahl der Wachepisoden pro Nacht). Für 5 Säuglinge lagen keine Angaben vor. Im Literaturvergleich zeigen die Ergebnisse sowohl Übereinstimmungen als auch Abweichungen zu anderen Untersuchungen. In Studien von Wolke, Meyer, Ohrt und Riegel (1995) sowie Fegert et al. (1997) zeigten nur rund 20\% der Kinder im Alter von ungefähr 5 und 20 Monaten häufige nächtliche Aufwachepisoden. Zuckerman et al. (1987) berichten über lediglich 10\% der Kindern ihrer 308-köpfigen Stichprobe im Alter von 8 Monaten, welche mehr als dreimal pro Nacht aufwachen. 
Ähnliche Ergebnisse zu der hier vorliegenden Untersuchung finden sich sowohl bei Studien von Gaylor et al. (2001) als auch bei Thunström et al (1999). Beide berichten analog von ungefähr einem Drittel der Kinder im Alter von 12 und 36 Monaten (Gaylor) und 6 bis 18 Monaten (Thunström), welche häufig nächtliche Wachepisoden angaben. Deutlich über den vorangegangenen Ergebnissen liegt die Untersuchung von Hiscock et al. (2001). Diese ermittelten $46 \%$ der Kinder ihrer Stichprobe im Alter von 6 bis 12 Monaten mit häufigen Aufwachepisoden (zwei Ereignisse) pro Nacht. So zeigt sich in der vergleichenden Betrachtung ein ungefähres Mittelmaß an nächtlichen Aufwachereignissen in der vorliegenden Stichprobe. Im Hinblick auf die Häufigkeitsverteilung der Nächte mit Wachepisoden pro Woche konnten keine vergleichbaren Studien gefunden werden. Es zeigte sich in der vorliegenden Stichprobe $(n=92)$, dass fast $43 \%$ der Kinder in mehr als 3 Nächten pro Woche Wachepisoden zu verzeichnen hatten (Kriteriumsvariable: Anzahl der Nächte mit Aufwachepisoden pro Woche).

Im Folgenden sollen zunächst die Merkmale diskutiert werden, welche einen denkbaren Zusammenhang zur kindlichen Schlafentwicklung haben, bevor die Erörterung der Hauptmerkmale „kindliches Temperament“ und „mütterliche Depressionsneigung und Ängstlichkeit" erfolgt. 


\subsection{Diskussion der Kontrollmerkmale}

\subsubsection{Arztkonsultationen}

Der Arztbesuch des Säuglings ist für viele Eltern die einzige Möglichkeit, Hilfe bei den Schlafproblemen ihres Kindes zu bekommen (Minde et al., 1993; Gaylor et al., 1998). Des Weiteren beschränkt sich das Schlafproblem nicht nur auf den Säugling, sondern es erstreckt sich nahezu ins gesamte familiäre Umfeld (Lozoff et al., 1985). Anhand der untersuchten Säuglinge wurde geprüft, ob eine Beziehung zwischen der Häufigkeit der Arztbesuche - ausgenommen die Vorsorgeuntersuchungen - und dem Schlafverhalten des Kindes im Alter von 12 Monaten besteht. Um mögliche Störfaktoren in Gestalt von chronischen Krankheiten bzw. Gesundheitseinschränkungen, welche schon zum Zeitpunkt der Geburt bestanden, ausschließen zu können, wurden lediglich gesunde Säuglinge von erstgebärenden Müttern in die Studie aufgenommen.

Es konnte bei den Säuglingen der Stichprobe während aller drei Erhebungszeiträume (I: Geburt bis 4. Monat, II: zwischen 4. und 8. Monat sowie III: zwischen 8. und 12. Monat) ein nahezu identischer Verlauf bezüglich der Anzahl der Arztbesuche festgestellt werden. So lag die durchschnittliche Häufigkeit während aller drei Zeiträume zwischen 1,3 und 1,6 Arztbesuchen, wobei die Extremwerte bei keinem bzw. bei 9 (Geburt bis 4. Monat sowie zwischen 8. und 12. Monat) und 15 (zwischen 4. und 8. Monat) Konsultationen lagen.

In Anbetracht vergleichbarer Studien, welche kindliche Regulationsstörungen und damit vergesellschaftete Schlafprobleme als eine der häufigsten Gründe für die Vorstellung in kinderärztlichen Praxen angeben (vgl. Wolke et al., 2002), werden auch die hier vorliegenden Ergebnisse interpretiert.

Ein eindeutiger Zusammenhang zwischen den Arztbesuchen und dem Schlafverhalten wurde in den ersten 4 Lebensmonaten nicht ersichtlich. Erst in der darauffolgenden Zeit kristallisierten sich deutliche Merkmalsbeziehungen sowohl bei der Aufwachhäufigkeit pro Nacht im Alter von 12 Monaten als auch bei der Häufigkeit der Nächte mit Wachepisoden pro Woche im Alter von 12 Monaten mit der Anzahl der Arztbesuche. Es wurde deutlich, dass Säuglinge, welche in der Zeit vom 4. bis zum 8. Lebensmonat häufig dem Arzt vorgestellt wurden, sowohl häufiger pro Nacht als 
auch in mehr Nächten pro Woche Wachepisoden aufwiesen als Kinder, welche kaum ärztliche Hilfe in Anspruch nahmen.

Für den Zeitraum zwischen dem 8. und 12. Monat konnte kein Zusammenhang zu den kindlichen Schlafgewohnheiten am Ende des ersten Jahres nachgewiesen werden.

Das erst ab dem 4. Lebensmonat deutlich werdende Beziehungsgefüge zwischen der Anzahl der Arztbesuche und dem Schlafverhalten des Kindes wird bei Betrachtung der Erkenntnisse Wolkes et al. (1998) und Skuses et al. (1994) ersichtlich. Nach ihrer Auffassung ist die Häufigkeit der nächtlichen Aufwachepisoden des Säuglings besonders in den ersten sechs Monaten an die Frequenz der Nahrungsaufnahme sowie an die enorme Wachstumsgeschwindigkeit gekoppelt.

Die Anzahl der Arztbesuche verhält sich, wie oben beschrieben, zwar in beiden Zeiträumen konstant, jedoch kann die erst später auftretende Merkmalsbeziehung damit begründet werden, dass in den ersten Lebensmonaten das kindliche Aufwachen nicht als Schlafproblem empfunden wird, sondern als notwendige Unterbrechung zur Nahrungsaufnahme des Kindes. Das Schlafproblem stellt sich hier als relativ stabil dar. Erst wenn sich die Nahrungsaufnahme des Kindes im Verlauf der weiteren Lebensmonate (4. bis 8. Lebensmonat) auf die Tageszeit konzentriert, werden die Aufwachepisoden des Nachts als Störung empfunden, und es entwickelt sich ein signifikantes Gefüge zwischen den nun wahrgenommenen Wachepisoden und den damit verbundenen Arztbesuchen.

Andererseits können im zweiten Lebenshalbjahr häufiger auftretende Erkältungskrankheiten eine mögliche Begründung für die Schlafprobleme zu dieser Zeit sein.

\subsubsection{Körpergewicht des Säuglings}

Im Rahmen der Studie wurden Korrelationen des Körpergewichtes der Säuglinge zum Zeitpunkt der Geburt sowie bei den Vorsorgeuntersuchungen U3 (im Alter von 4 bis 6 Wochen), U5 (im Alter von 6 bis 7 Monaten) und U6 (im Alter von circa 1 Jahr) und dem Schlafverhalten im Alter von 12 Monaten festgestellt.

Richtet man sich nach der einschlägigen Literatur, so stellt sehr geringes Geburtsgewicht $(<2500 \mathrm{~g})$ einen risikoerhöhenden Faktor für die kindliche Entwicklung dar (Laucht et al., 1998). Weiterhin wird in der Literatur ein Zusammenhang zwischen 
einer schwachen Gewichtszunahme (bei normalem Geburtsgewicht und Gesundheit) während der ersten 6 Monate und einer daraus resultierenden ungünstigeren kognitiven Entwicklung erwähnt (Skuse et al., 1994).

Eines der Ausschlusskriterien der Studie war ein Geburtsgewicht unterhalb $2500 \mathrm{~g}$. Somit wurden extrem untergewichtige Säuglinge hier nicht untersucht. Das durchschnittliche Geburtsgewicht betrug in der Studie circa $3415 \mathrm{~g}$. Es zeigte sich bei Säuglingen mit geringem Gewicht ein signifikanter Zusammenhang mit deutlich erhöhten Aufwachepisoden pro Nacht im Alter von 12 Monaten. In der weiteren Durchführung der Studie zeigte sich bei der Auswertung der Ergebnisse bezüglich des Körpergewichtes bei den jeweiligen Vorsorgeuntersuchungen eine Fortsetzung der negativen Korrelation auf signifikantem Niveau. Eine Ausnahme bildete lediglich die Vorsorgeuntersuchung U5. Es zeigt sich sowohl in der 4. bis 6. Lebenswoche (U1) als auch im Alter von einem Jahr (U6), dass der körperliche Reifezustand des Säuglings in Gestalt des Körpergewichtes als Prädiktor für die nächtliche Aufwachhäufigkeit des Kindes gesehen werden kann. So könnte ein Säugling mit niedrigem Gewicht aufgrund seines Gewichtsdefizits auch nachts nach Nahrung verlangen, weshalb die Schlafunterbrechung klinisch als „physiologische Anpassung“ aufgefasst werden kann. Andererseits könnte das geringe Gewicht, wie auch Laucht et al. (1998) und Skuse et al. (1994) schon beschreiben, zu einer geringeren Reifeentwicklung und somit zu Regulationsstörungen führen, welche sich als Schlafproblem bemerkbar machen. In Studien von Huizink et al. (2004) zeigte sich subklinisch, dass schon pränataler Stress der Mutter zur vermehrten Stresshormonausschüttung führt und dies über verschiedene biologische Regelkreisläufe das Geburtsgewicht des Kindes mindert. Im Weiteren hat dies Entwicklungsdefizite des Kindes zur Folge und damit verbunden auch Schlafprobleme.

\subsubsection{Stilldauer und Stillverhalten}

Die Einbeziehung des Stillverhaltens in die Untersuchung sollte Aufschluss darüber geben, ob sich anhand der Stilleigenschaften und der Stilldauer Einflussfaktoren auf das Schlafverhalten des Säuglings mit 12 Monaten aufzeigen lassen.

Bei den 91 untersuchten Säuglingen der Stichprobe lag die durchschnittliche Stilldauer bei 27,46 Wochen. Dabei gab es Mütter $(n=3)$, welche nur kurz (1-2 Wochen) 
ihr Kind stillten bzw. Mütter ( $n=12)$, welche dies noch über den letzten Befragungstermin hinaus im Alter von 12 Monaten machten. Im Rahmen der vorliegenden Studie zeigte sich zu keinem Zeitpunkt des Verlaufs eine auch nur annähernde Korrelation zwischen dem Schlafverhalten und dem Stillverhalten, auch dann nicht, wenn das kindliche Körpergewicht einbezogen wurde. So ergab sich auch kein Zusammenhang zwischen Stillverhalten und Körpergewicht.

Dieses Ergebnis steht im Widerspruch zur Literatur. Hier berichten etliche Studien von deutlich häufigeren und längeren Wachepisoden bei Säuglingen, welche von der Mutter gestillt wurden. Hiscock et al. (2002) wiesen in ihrer Studie bei circa $60 \%$ brustgestillter Kinder gehäufte Schlafproblematiken nach im Gegensatz zu nur 40 \% flaschengestillter Kinder. Ähnliche Ergebnisse zeigen Wolke et al. (1998) und Thunström (2002), die in ihren Studien ebenfalls vermehrt Schlafschwierigkeiten bei brustgestillten als bei flaschengestillte Kinder nachweisen konnten.

Weiter stellen Wolke et al. (1998) anhand ihrer Untersuchung an insgesamt 5484 Kindern das Bruststillen sogar als einzigen unabhängigen Prädiktor für Schlafprobleme im 5. Lebensmonat dar.

Die hier untersuchte Stichprobe stellt sich im Vergleich zu den anderen Stichproben zahlenmäßig als viel kleiner dar, so dass sich unter Umständen sehr kleine Effekte nicht zeigen lassen. Weiter scheint auch das im vorangegangenen Abschnitt erläuterte Gewicht des Kindes als entscheidender im Hinblick auf das Schlafverhalten als auf das Stillen bzw. Nicht-Stillen.

\subsubsection{Kognitive Entwicklung des Säuglings}

Mit Hilfe des „Bayley Mental Tests“ (Bayley, 1993) wurden die Säuglinge in dieser Studie hinsichtlich ihrer kognitiven Entwicklung, ihrer Anpassungsleistungen, ihrer sensorischen Reaktionen und auch bezüglich ihrer Aufmerksamkeit für Stimuli überprüft.

In der gängigen Literatur konnten keine Ergebnisse gefunden werden, welche einen Zusammenhang der kognitiven Reife mit den kindlichen Schlafproblemen nahelegen. In unseren Ergebnissen fanden sich teils positive, teils negative Korrelationen. Im 8. Lebensmonat stellt sich die kognitive Entwicklung des Kindes in negativer Korrelation mit den kindlichen Schlafproblemen dar. Es zeigt sich, dass Kinder mit einem über- 
durchschnittlichen Mental Developmental Index (MDI) zum Zeitpunkt des 8. Lebensmonats später mit einem Jahr weniger pro Nacht aufwachen. Hier prädiziert ein im Alter von 8 Monaten hoher kognitiver Reifestand eine geringere Aufwachfrequenz pro Nacht 4 Monate später.

Im Alter von 12 Monaten zeigt sich indes eine hochsignifikante positive Korrelation zwischen häufigen Nächten mit Aufwachepisoden pro Woche und der kognitiven Entwicklung des Kindes zum Zeitpunkt des 12. Lebensmonats. Dies bedeutet: Ein Säugling, der zum Untersuchungszeitpunkt im 12. Lebensmonat eine überdurchschnittliche kognitive Entwicklung zeigt, wacht zur gleichen Zeit auch regelmäßiger pro Woche auf als Säuglinge mit niedrigeren Werten. So könnte der „intelligente“ Säugling viele Reize aufnehmen und damit verbundene eine höhere Aktivation angenommen werden, welche als Resultat eine vermehrte Aufwachhäufigkeit pro Woche mit sich bringt. Weiter könnte dem Zusammenhang ein erlerntes Verhalten des Kindes und ein bestimmtes Erziehungsverhalten der Eltern (viel Stimulation und Reaktivität gegenüber dem Kind) zugrunde liegen. Kinder mit einem hohen Mental Developmental Index (MDI) wachen regelmäßig (viele Nächte pro Woche) auf, aber nicht sehr häufig in einer Nacht. Es könnte sein, dass die Eltern dieser Kinder auch nachts bereit sind auf das Verhalten ihres Nachwuchses zu reagieren.

\subsubsection{Säuglingskoliken}

Insgesamt konnten alle 98 Säuglinge aus der Stichprobe über den von der Mutter ausgefüllten Fragebogen auf ihre Kolikausprägung in Form von „Perioden unberuhigbaren Schreiens“ untersucht werden. Im Verlauf der beiden Untersuchungszeiträume (bis zum 4. Lebensmonat und im 4. Lebensmonat) zeigte sich ein deutlicher Rückgang der Koliken von $51 \%$ auf nur noch $17 \%$ im 4. Lebensmonat bei Kindern, welche in den ersten Lebensmonaten „zeitweise“ bis „deutliche“ Koliken aufwiesen. Noch offensichtlicher wird dieser Trend bei der Betrachtung der Säuglinge mit sehr häufigen Koliken. Dort zeigt sich eine Abnahme der Häufigkeit von $29 \%$ in den ersten Monaten auf nahezu $3 \%$ im 4. Lebensmonat.

Ein deutlicher Zusammenhang war auch feststellbar zwischen der Ausprägung der Koliken in der Zeit bis zum 4. Lebensmonat und der Anzahl der Nächte mit Wachepi- 
soden pro Woche am Ende des ersten Lebensjahres. Im Alter von 4 Monaten bestand hier kein Zusammenhang mehr. Mit der nächtlichen Aufwachhäufigkeit ergab sich ein weiterer signifikanter Zusammenhang. Säuglinge, bei denen trotz des beschriebenen allgemeinen Rückgangs der Koliken diese weiterhin angegeben wurden, zeigten im Alter von 12 Monaten häufige Wachepisoden pro Nacht.

Das gewonnene Ergebnis entspricht durchaus den Erkenntnissen der vorhandenen Literatur. St. James-Roberts et al. (1997) zeigen in ihren Studien, dass Kinder mit Koliken in Form von andauernden Schreiphasen besonders im Alter von 6 Wochen eine kürzere Gesamtschlafzeit haben als gemäßigte Schreikinder. Sowohl Wolke (1994) als auch v. Hofacker et al. (1996) beschreiben in ihren Studien an Kindern, welche im Alter von 1 bis 6 Monaten wegen exzessiven Schreiens vorgestellt wurden, dass jeweils circa 70 \% der Schreikinder ein gleichzeitiges Schlafproblem besaBen. Kirjavainen et al. (2001) verzeichnen in ihrer Studie bezüglich kindlicher Koliken und Schlafverhalten auch eine vermehrte Aufwachhäufigkeit in den ersten Lebenswochen, allerdings nur anhand eines Tagebuches von elterlicher Seite, in welchem das Schlafverhalten dokumentiert wurde. Demgegenüber weisen die gleichen Säuglinge bei der Beobachtung im Schlaflabor (Ausnahme: Zeitpunkt der Koliken) im Alter von 2 und 7 Monaten keinen signifikanten Unterschied zum Schlafverhalten von Kindern der Kontrollgruppe auf.

Aufgrund der hier gewonnenen Ergebnisse kann vermutet werden, dass die Kinder, welche mit 4 Monaten Koliken aufweisen, auch später im Alter von 12 Monaten schlecht schlafen und damit verbunden ihre Eltern wecken. Dies zeigen ebenfalls einige weitere Studien ungeachtet der angewandten Methoden. 


\subsection{Diskussion der Hypothesen}

Im nachfolgenden Abschnitt sollen die gewonnenen Ergebnisse zu den Hauptmerkmalen „Temperament des Säuglings“ und „Depressionsneigung und Ängstlichkeit der Bezugsperson" im Zusammenhang mit dem kindlichen Schlafverhalten im Alter von 12 Monaten diskutiert und erörtert werden.

\subsubsection{Temperamentsmerkmale und Schlafverhalten}

\subsubsection{Positive Emotionalität und Schlafverhalten}

Laucht et al. (1997) weisen positiven kindlichen Temperamentsmerkmalen in Form von einem flexiblen, aktiven und offenen Verhalten einen wesentlichen protektiven Mechanismus bei Begegnungen mit Störfaktoren zu.

In der hier erhobenen Studie zeigt sich an den untersuchten Säuglingen eine deutlich protektive Wirkung der positiven Emotionalität hinsichtlich der nächtlichen Aufwachhäufigkeit im Alter von 12 Monaten. So weist ein Kind mit häufigem Lachen/Lächeln (erfasst anhand der Fragebogenskala) und ausgeprägter positiver Emotionalität (erfasst anhand des Verhaltenstests) zu fast allen drei Erhebungszeitpunkten am Ende des ersten Lebensjahres weniger nächtliche Aufwachepisoden auf als ein Kind mit geringer ausgeprägter positiver Emotionalität. Folglich kann die positive Emotionalität auf kindlicher Seite als protektiv im Hinblick auf die Aufwachsymptomatik dargestellt werden.

Dieses Ergebnis findet zusätzliche Bestätigung durch Untersuchungen Sroufes (1996), die verdeutlichen, dass positive Emotionalität eine ausgeprägte Resilienz, gute Anpassungsfähigkeit, Emotionsregulation und die Rhythmizität von Bioregulation indiziert.

\subsubsection{Negative Emotionalität und Schlafverhalten}

Bei der Untersuchung der Stichprobe an Säuglingen, welche vermehrt Irritierbarkeit und Unbehagen bei Frustration/Einschränkung (erfasst anhand der Fragebogenska- 
la) und negative Emotionalität (erfasst anhand des Verhaltenstests) aufwiesen, kann ebenfalls eine Literaturübereinstimmung erreicht werden, wenn auch nicht auf dem vorangegangenem hohen Niveau.

Scheithauer et al. (2000) stellen Merkmale wie vermehrte Ablenkbarkeit, schwieriges Temperament und verminderte Intelligenz als klare endogene Risikofaktoren für den heranwachsenden Säugling dar.

Ein signifikantes Ergebnis konnte mit Ausnahme des 8. Lebensmonats zu allen Erhebungszeitpunkten (4. und 12. Monat) in der Gruppe der Säuglinge mit vermehrt negativer Emotionalität gefunden werden.

Es zeigte sich eine tendenzielle Beziehung zwischen der vermehrten negativen Emotionalität und Irritierbarkeit des Säuglings im Alter von 4 Monaten und der Häufigkeit der Nächte mit Aufwachepisoden pro Woche am Ende des ersten Lebensjahres. Im Alter von 12 Monaten bestand eine deutlich höhere Anzahl an nächtlichen Aufwachepisoden bei Säuglingen, welche im Verhaltenstest hohe negative Emotionalität zeigten als bei Säuglingen mit geringer negativer Emotionalität.

Die ausgebliebene Korrelation zum Zeitpunkt des 8. Monats kann mit der hohen Dynamik und Reifungsgeschwindigkeit dieses Zeitpunktes bezüglich der Differenzierung des negativen Affektausdrucks in Emotionen wie Angst und Ärger (Sroufe, 1996) begründet werden.

\subsubsection{Zusammenfassung}

Nach der Betrachtung der Temperamentsmerkmale „positive und negative Emotionalität" wird deutlich, dass beide Merkmalsausprägungen mit Schlafproblementwicklung verbunden sind. Es zeigten sich bei schlecht schlafenden Kindern erhöhte negative Reaktionen und eine geringere Anpassungsleistung.

\subsubsection{Mütterliche Merkmale und kindliches Schlafverhalten}

Im ersten Lebensjahr ist die Unterstützung der Bezugsperson unbedingt erforderlich und entscheidend für die beginnende Selbstregulation des Kindes (Kopp, 1989; Sroufe, 1996; Thompson, 1994). So wurde innerhalb der hier vorliegenden Studie die 
These geprüft, ob mütterliche Befindlichkeitsmerkmale mit der Entwicklung des kindlichen Schlafverhaltens assoziiert sind.

Es zeigte sich innerhalb der untersuchten Säuglinge besonders zum Untersuchungszeitpunkt von 4 Monaten eine offensichtliche Merkmalsbeziehung zwischen der mütterlichen Depressivität und Ängstlichkeit und dem Schlafverhalten der Kinder im Alter von 12 Monaten. Deutlich wurde dies bei der Auswertung der Skala „Hoffnungslosigkeit" von Beck in der deutschen Bearbeitung von Krampen (1979). Hierbei zeigte sich, dass Mütter, welche vermehrt negative Einstellungen über sich selbst, ihre Umwelt und die Zukunft äußerten, später häufiger über nächtliche Schlafprobleme des Kindes berichteten. Anzumerken ist, dass eher die Hoffnungslosigkeit eine Rolle spielt als die Ängstlichkeit der Mütter, welche keine signifikanten Ergebnisse bei der Auswertung der Skala „Trait-Angst“ aus dem „State-Trait-Angstinventar“ (STAI) von Laux, Glanzmann, Schaffer und Spielberger (1981) aufzeigte. Nur bei Müttern, welche zum Zeitpunkt des 8. Monats keine Überfürsorge aus Angst angaben, zeigte sich eine weitere Beziehung zum Schlafverhalten im Alter von 12 Monaten. So schliefen solche Kinder im Alter von einem Jahr besser, deren mütterliche Interaktion nicht von Überängstlichkeit geprägt war.

Die Beziehung zwischen mütterlichen Befindlichkeitsmerkmalen und dem kindlichen Schlafverhalten wird in der Skala „Depressivität“ aus dem „Fragebogen zur Erfassung der Erziehungseinstellungen von Müttern mit Kindern im Kleinstkindalter“ (EMKK) von Engfer (1984) dargestellt. Hier zeigte sich wiederum im Alter von 4, aber auch von 12 Monaten eine klare Abhängigkeit der depressiven Neigung auf mütterlicher Seite und dem vermehrten nächtlichen Aufwachen der Kinder zum Ende des ersten Lebensjahres.

Zu diskutieren ist hier die Frage, warum die mütterliche Depressivität/ Ängstlichkeit besonders zum Zeitpunkt des 4. Lebensmonats eine so deutliche Beziehung zum Schlafverhalten des Kindes zeigt und sich zu späteren Zeitpunkten als weniger entscheidend darstellt. Dies lässt den Schluss zu, dass Säuglinge im Alter von 4 Monaten besonders sensibel auf mütterliche Depressivität und Ängstlichkeit reagieren. Eine ähnliche Meinung vertreten Murray \& Cooper (1997). Sie beschränken den Einfluss der mütterlichen Depressivität lediglich auf die frühe Kindheitsphase. Bei ihrer Untersuchung von Kleinkindern im fortgeschrittenerem Alter sowie Vorschulkindern 
konnte sich die Depression der Mutter als alleiniges Merkmal zur Beeinflussung des kindlichen Verhaltens nicht halten.

Aufgrund der hier erhobenen Studie ist im Weiteren davon auszugehen, dass sich die frühe Phase der Kindheit als besonders sensitiv für Depressivität und Ängstlichkeit von Seiten der Mutter darstellt. Dies wurde durch die vermehrten Aufwachepisoden im Alter von 12 Monaten bestätigt.

Eine Beziehung zu späteren Zeitpunkten bzw. zu der Häufigkeit der Nächte mit Aufwachepisoden pro Woche konnte mit den Ergebnissen dieser Studie nicht bestätigt werden.

\subsubsection{Zusammenfassung}

Die Zusammenhänge von mütterlicher Depressivität und Ängstlichkeit und kindlichem Schlafverhalten in Form nächtlicher Aufwachhäufigkeit konnte im Rahmen dieser Studie und Literaturvergleiche vorrangig im Alter von 4 Monaten bestätigt werden. Kinder von Müttern mit depressiven Neigungen zum Untersuchungszeitpunkt 4. Monat tendieren demzufolge zu vermehrten nächtlichen Aufwachepisoden im Alter von 12 Monaten. Weiter zeigte sich das kindliche Verhalten in den ersten vier Monaten als besonders sensibel im Hinblick auf die mütterlichen Befindlichkeitsmerkmale. Ferner war feststellbar, dass sich das kindliche Verhalten besonders in den ersten vier Monaten als vulnerabel bezüglich des mütterlichen Gemüts verhält. Im weiteren Entwicklungsverlauf erwies es sich als weniger beeinflussbar von Gefühlsmerkmalen der Bezugsperson.

\subsection{Ausblick}

Abschließend sollen die in dieser Studie gewonnenen Ergebnisse zusammengefasst, diskutiert und mögliche Ansatzpunkte für weitere Untersuchungen aufgezeigt werden.

Interessanterweise zeigte sich, dass 4/5 der als signifikant verknüpften Variablen (Hypothesen- und Kontrollvariablen) mit dem Parameter „Anzahl der Aufwachepisoden pro Nacht“ im Zusammenhang stehen. Lediglich 1/5 der signifikant verknüpften 
Variablen zeigten einen Zusammenhang mit dem Parameter „Nächte pro Woche mit Aufwachepisoden“ (vgl. Ergebnisteil Tabelle 16). Betrachtet man in diesem Zusammenhang die Definition Wolkes (1999) über Schlafstörungen, so zeigt sich, dass dieser sowohl die Anzahl der Tage pro Woche, an denen das Kind aufwacht als auch die Häufigkeit an Aufwachepisoden pro Nacht in seine Beurteilung über das kindliche Schlafverhalten mit einbezieht.

Die Ergebnisse der hier vorliegenden Untersuchung machen zwar deutlich, dass signifikante Merkmalsbeziehungen zu beiden Variablen („Anzahl der Aufwachepisoden pro Nacht“ \& „Nächte pro Woche mit Wachepisoden“) vorliegen, die Gewichtung liegt jedoch deutlich auf Seiten der „Anzahl der Aufwachepisoden pro Nacht“.

Folglich lässt sich der erste Parameter („Anzahl der Aufwachepisoden pro Nacht“ im Alter von 12 Monaten) in größerem Maße durch kindliche Faktoren (positive/negative Emotionalität) und durch mütterliche Faktoren (Depressionsneigung/Ängstlichkeit) vorhersagen als der zweite Parameter („Nächte pro Woche mit Wachepisoden“ im Alter von 12 Monaten).

Eine Aufgabe weiterer Studien könnte damit sein zu kontrollieren, ob sich die Aufwachhäufigkeit pro Nacht bezüglich der Schlafprobleme wirklich als entscheidenderer Faktor im Gegensatz zur Anzahl der Nächte pro Woche mit Wachepisoden darstellen lässt. Weiter wäre zu prüfen, ob eine frühzeitige Intervention durch eine pädiatrische Beratung und eine psychotherapeutische Unterstützung sowohl kindliche als auch mütterliche Faktoren in solchem Maße beeinflussen kann, dass die Schlafproblematik in Form von häufigen nächtlichen Aufwachepisoden deutlich reduziert werden könnte. 


\section{Zusammenfassung}

Schlafstörungen in der frühen Kindheit stellen ein weit verbreitetes Problem dar und gehören zu den häufigsten Vorstellungsgründen von Kindern in der pädiatrischen Praxis.

Da besonders für das erste Lebensjahr zur kindlichen Schlafentwicklung und der Entstehung von Regulationsstörungen nur sehr wenig Studien vorhanden sind und bisher nur selten unausgelesene Stichproben gesunder Mutter-Kind Paare untersucht wurden, geht die vorliegende Arbeit der Frage nach, unter welchen Bedingungen sich Schlafproblematiken respektive Regulationsstörungen entwickeln und wie sie möglichst frühzeitig erkannt werden können. Hierzu werden im Rahmen der vorliegenden Arbeit Merkmale auf kindlicher und mütterlicher Seite zu drei vorgegebenen Untersuchungszeitpunkten (4., 8. und 12. Monat) hinsichtlich ihres Vorhersagewertes auf kindliche Schlafprobleme im 12. Lebensmonat geprüft. Vorrangig werden Beziehungen zwischen kindlichem Temperament und mütterlicher Depressionsneigung/ Ängstlichkeit mit möglichen Schlafproblematiken am Ende des ersten Lebensjahres untersucht. Von untergeordneter Bedeutung werden als Kontrollmerkmale die Arztbesuche des Kindes, das Körpergewicht, die Stilldauer und das Stillverhalten, die kognitive Reife sowie die Häufigkeit von Koliken mit den Schlafproblematiken am Ende des ersten Lebensjahres betrachtet.

Die Studie wurde an insgesamt 101 Bezugspersonen-Kind-Paaren durchgeführt. 3 Bezugspersonen-Kind-Paare wurden aufgrund eines Wechsels der primären Bezugsperson im Verlauf der Studie ausgeschlossen.

Es zeigten $61 \%$ der Säuglinge 0 bis 1 Wachepisoden pro Nacht (18:00 bis 6:00 Uhr), 34\% 2 oder mehr Wachepisoden pro Nacht (18:00 bis 6:00 Uhr). Für 5 Säuglinge lagen keine Angaben vor. Bei der Betrachtung der Aufwachhäufigkeit pro Woche zeigte sich, dass $51 \%$ der Säuglinge an weniger als 2 Nächten pro Woche Durchschlafprobleme aufwiesen, 43 \% jedoch an 3 oder mehr Nächte der Woche Durchschlafprobleme hatten. Für 6 Säuglinge lagen keine Angaben vor.

Die notwenigen Daten zum kindlichen Schlafverhalten und Temperament sowie der Depressivität/ Ängstlichkeit der Bezugsperson als auch der Kontrollmerkmale wurden anhand von strukturierten Interviews mit der Bezugsperson, Fragebögen und standardisierten Verhaltenstests erfasst. 
Es stellten sich folgende Zusammenhängen als signifikant dar:

Kinder mit wenigen nächtlichen Aufwachepisoden im Alter von einem Jahr zeigten durchweg eine höhere positive Emotionalität im Urteil der Mutter zum Untersuchungszeitpunkt im 4., 8. und 12. Monat und im Verhaltenstest im 8. und 12. Monat. Weiterhin beschrieben sich die Mütter der schlecht schlafenden Kinder zum Zeitpunkt des 4. und 12. Monates als depressiver, zum Zeitpunkt des 4. und 8. Monats als ängstlicher sowie zum Zeitpunkt des 4 Monats als hoffnungsloser als die Mütter der Kinder ohne Schlafprobleme.

Anhand der Kontrollmerkmale zeigten die Kinder mit Schlafproblemen häufigere Arztbesuche (ausgenommen die Vorsorgeuntersuchungen), ein geringeres Körpergewicht und mehr Koliken (Perioden unberuhigbaren Schreiens) im Vergleich zu den Kindern, welche gut schliefen.

Bezogen auf die Anzahl der Nächte pro Woche, in denen das Kind nachts aufwacht, ergab sich, dass jene Kinder häufiger pro Woche aufwachten, die vermehrt Unbehagen bei Frustration/Einschränkung zeigten, häufiger den Arzt konsultierten, eine höhere kognitive Reife besaßen sowie über vermehrte Koliken klagten.

Es konnten keine Merkmalsbeziehungen zwischen Stilldauer und Stillverhalten mit den Schlafgewohnheiten im Alter von 12 Monaten nachgewiesen werden. 


\section{Summary}

Sleeping disorders in the early infanthood is a widespread problem, and belongs to the most frequent consultation reasons for children in the pediatric practice.

As there are only very few studies concerning the sleep development and the onset of regulation disorders in the first year of life, and only very few representative control samples of healthy mother-child couples were examined, this doctorate researches the circumstances under which sleeping problems and respective regulation disorder develop and how to detect them early.

This doctorate spans the maternal and infantile features of three examinations points (4, 8 and 12 months), and compares their results in the 12th month. The primary focus is on the relation between the infantile vivacity and maternal tendency to depression/anxiety with possible sleeping problems at the end of the first year. Therefore, of secondary significance, the control features of medical consultations of the child, its bodyweight, the cognitive maturity, as well as the frequency of colics with the sleeping problems are followed at the end of the year.

The study was performed on altogether 101 mother-child couples; 3 of such couples were to be excluded, as the primary attachment figure changed. In view of the infants, $61 \%$ showed 0 to 1 wake-up episodes per night (06:00 pm - 06:00 am), $34 \%$ 2 or more of such episodes. There was no data available for 5 infants. In view of the frequency in waking up per week, $51 \%$ of the infants had problems with sleeping through less than 2 nights per week; however, $43 \%$ had sleeping problems 3 or more nights a week. For altogether 6 infants there was no information available. The necessary data on the infantile sleeping performance and vivacity, as well as the depression/anxiety of the attachment figure, and, the control features were gathered in structured interviews with the attachment figure, questionnaires and standardized behavioral tests.

The following significance was evaluated:

According to their mothers, infants of age 1 had few nightly wake-up episodes, continuously showed a higher positive emotionalism with 4, 8 and 12 months, and in the behavioral test with 8 and 12 months at the same age. Moreover, at the age of 4 and 12 months mothers of badly sleeping infants found themselves more depressive, more anxious with 4 and 8 months, and, more hopeless with 4 months. In compari- 
son to the well-sleeping infants, the control features revealed that children with sleeping problems were seen by medics more frequently (excluding the screenings and primings), had less bodyweight and more colics (periods of uncalmable screaming). Based on the amount per week, in which a child wakes up, the study showed that infants with augmented discomforts upon frustration/restrictions, medical consultations, higher cognitive maturity and colics that are more frequent wake up more often per week.

A relation between the duration of the breastfeeding phase and its performance with the sleeping habits at the age of 12 months could not be proved. 


\section{Anhang A: Tabellen}

Tabelle 1: $\quad$ Häufigkeitstabelle des Schlafverhaltens der Säuglinge

Tabelle 2: $\quad$ Gruppenvergleich (Chiquadrat-Tests) hinsichtlich der nächtlichen und wöchentlichen Aufwachhäufigkeit

Tabelle 3: $\quad$ Häufigkeitstabelle der Arztbesuche der Säuglinge

Tabelle 4: $\quad$ Zweigruppenvergleich (Chiquadrat-Tests) hinsichtlich der Arztbesuche

Tabelle 5: $\quad$ Häufigkeiten/ Statistiken zum Körpergewicht der Säuglinge

Tabelle 6: $\quad$ Pearson Rangkorrelationskoeffizienten des Körpergewichts und dem Schlafverhalten der Säuglinge

Tabelle 7: $\quad$ Häufigkeiten/ Statistiken zum Stillverhalten

Tabelle 8: $\quad$ Pearson Rangkorrelationskoeffizienten des Stillverhaltens und dem Schlafverhalten der Säuglinge

Tabelle 9: $\quad$ Spearman-Rho Rangkorrelationskoeffizienten des Stillverhaltens und dem Körpergewicht der Säuglinge

Tabelle 10: $\quad$ Pearson Rangkorrelationskoeffizienten der kognitiven Reife und dem Schlafverhalten der Säuglinge

Tabelle 11: $\quad$ Häufigkeitstabelle über Koliken der Säuglinge

Tabelle 12: $\quad$ Zweigruppenvergleich (Chiquadrat-Tests) hinsichtlich der Koliken

Tabelle 13: $\quad$ Pearson Rangkorrelationskoeffizienten der positiven Emotionalität und dem Schlafverhalten der Säuglinge

Tabelle 14: $\quad$ Pearson Rangkorrelationskoeffizienten der negativen Emotionalität und dem Schlafverhalten der Säuglinge

Tabelle 15: $\quad$ Zweigruppenvergleich (Chiquadrat-Tests) hinsichtlich der positiven Emotionalität

Tabelle 16: $\quad$ Zweigruppenvergleich (Chiquadrat-Tests) hinsichtlich der negativen Emotionalität

Tabelle 17: $\quad$ Pearson Rangkorrelationskoeffizienten der mütterlichen Merkmale und dem Schlafverhalten der Säuglinge

Tabelle 18: $\quad$ Häufigkeitstabelle hinsichtlich des Geschlechts der Säuglinge

Tabelle 19: $\quad$ Häufigkeitstabelle zum Alter der Mutter

Tabelle 20: $\quad$ Häufigkeitstabelle zum Alter des Vaters

Tabelle 21: Häufigkeitstabelle zum Bildungsniveau der Mutter

Tabelle 22: Häufigkeitstabelle zum Bildungsniveau des Vaters 


\begin{tabular}{|cc|}
\hline \multicolumn{2}{|c|}{ Legende } \\
$\mathrm{n}=$ Stichprobengröße & Anz. Fall $=$ Anzahl der Fälle \\
$\mathrm{p}=$ Irrtumswahrscheinlichkeit & Erw. Wert. = Erwartungswert \\
$\mathrm{r}=$ Korrelationskoeffizient & Zeilen $\%=$ Zeilenprozent \\
$\mathrm{ns}=$ nicht signifikant & Spalten $\%=$ Spaltenprozent \\
& $\mathrm{FG}=$ Freiheitsgrade \\
\hline
\end{tabular}


Tabelle 1: Häufigkeitstabelle des Schlafverhaltens der Säuglinge

Wachepisoden pro Nacht (18:00 bis 6:00 Uhr) im Alter von 12 Monaten

\begin{tabular}{|c|r|r|}
\hline & Häufigkeit & Prozent \\
\hline 0 & 15 & 15,3 \\
1 & 45 & 45,6 \\
2 & 17 & 17,3 \\
3 & 10 & 10,8 \\
4 & 4 & 4,1 \\
6 & 1 & 1,0 \\
8 & 1 & 1,0 \\
Gültig Ge- & 93 & 94,9 \\
samt & & \\
Fehlend & 5 & 5,1 \\
GESAMT & 98 & 100 \\
\hline
\end{tabular}

Nächte mit Wachepisoden pro Woche im Alter von 12 Monaten

\begin{tabular}{|c|r|r|}
\hline & Häufigkeit & Prozent \\
\hline 0 & 26 & 26,5 \\
1 & 13 & 13,3 \\
2 & 11 & 11,2 \\
3 & 7 & 7,1 \\
4 & 4 & 4,1 \\
5 & 3 & 3,1 \\
7 & 28 & 28,6 \\
Gültig Ge- & 92 & 93,9 \\
samt & & \\
Fehlend & 6 & 6,1 \\
GESAMT & 98 & 100 \\
\hline
\end{tabular}


Tabelle 2: Gruppenvergleich hinsichtlich der nächtlichen und wöchentlichen Aufwachhäufigkeit (Chiquadrat-Tests)

\begin{tabular}{|c|c|c|c|c|c|c|c|c|c|}
\hline \multirow{2}{*}{\multicolumn{2}{|c|}{$\begin{array}{l}\text { Anz. Fall } \\
\text { Erw. Wert } \\
\text { Zeilen \% } \\
\text { Spalten \% }\end{array}$}} & \multicolumn{7}{|c|}{ Nächte mit Aufwachepisoden pro Woche } & \multirow{2}{*}{$\begin{array}{c}\text { Zeile } \\
\text { ge- } \\
\text { samt }\end{array}$} \\
\hline & & 0 & 1 & 2 & 3 & 4 & 5 & 7 & \\
\hline \multirow{28}{*}{$\begin{array}{l}\frac{1}{0} \\
\frac{\pi}{2} \\
\frac{0}{2} \\
\frac{0}{2} \\
\frac{1}{0} \\
\frac{0}{0} \\
\frac{.0}{0} \\
\frac{0}{0} \\
\frac{1}{0} \\
\frac{\pi}{3} \\
\frac{1}{2}\end{array}$} & 0 & 15 & 0 & 0 & 0 & 0 & 0 & 0 & 15 \\
\hline & & 4,2 & 2,1 & 1,8 & 1,1 & 0,7 & 0,5 & 4,6 & 15,0 \\
\hline & & 100 & 0 & 0 & 0 & 0 & 0 & 0 & 100 \\
\hline & & 57,7 & 0 & 0 & 0 & 0 & 0 & 0 & 16,3 \\
\hline & 1 & 8 & 8 & 7 & 7 & 3 & 1 & 10 & 44 \\
\hline & & 12,4 & 6,2 & 5,3 & 3,3 & 1,9 & 1,4 & 13,4 & 44,0 \\
\hline & & 18,2 & 18,2 & 15,9 & 15,9 & 6,8 & 2,3 & 22,7 & 100 \\
\hline & & 30,8 & 61,5 & 63,6 & 100 & 75 & 33,3 & 35,7 & 47,8 \\
\hline & 2 & 3 & 3 & 2 & 0 & 1 & 0 & 8 & 17 \\
\hline & & 4,8 & 2,4 & 2,0 & 1,3 & 0,7 & 0,6 & 5,2 & 17 \\
\hline & & 17,6 & 17,6 & 11,8 & 0 & 5,9 & 0 & 47,1 & 100 \\
\hline & & 11,5 & 23,1 & 18,2 & 0 & 25 & 0 & 28,6 & 18,5 \\
\hline & 3 & 0 & 2 & 2 & 0 & 0 & 2 & 4 & 10 \\
\hline & & 2,8 & 1,4 & 1,2 & 0,8 & 0,4 & 0,3 & 3,0 & 10 \\
\hline & & 0 & 20 & 20 & 0 & 0 & 20 & 40 & 100 \\
\hline & & 0 & 15,4 & 18,2 & 0 & 0 & 66,7 & 14,3 & 10,9 \\
\hline & 4 & 0 & 0 & 0 & 0 & 0 & 0 & 4 & 4 \\
\hline & & 1,1 & 0,6 & 0,5 & 0,3 & 0,2 & 0,1 & 1,2 & 4,0 \\
\hline & & 0 & 0 & 0 & 0 & 0 & 0 & 100 & 100 \\
\hline & & 0 & 0 & 0 & 0 & 0 & 0 & 14,3 & 4,3 \\
\hline & 6 & 0 & 0 & 0 & 0 & 0 & 0 & 1 & 1 \\
\hline & & 0,3 & 0,1 & 0,1 & 0,1 & 0 & 0 & 0,3 & 1 \\
\hline & & 0 & 0 & 0 & 0 & 0 & 0 & 100 & 100 \\
\hline & & 0 & 0 & 0 & 0 & 0 & 0 & 3,6 & 1,1 \\
\hline & 8 & 0 & 0 & 0 & 0 & 0 & 0 & 1 & 1 \\
\hline & & 0,3 & 0,1 & 0,1 & 0,1 & 0 & 0 & 0,3 & 1 \\
\hline & & 0 & 0 & 0 & 0 & 0 & 0 & 100 & 100 \\
\hline & & 0 & 0 & 0 & 0 & 0 & 0 & 3,6 & 1,1 \\
\hline \multirow{4}{*}{\multicolumn{2}{|c|}{$\begin{array}{l}\text { Spalte } \\
\text { gesamt }\end{array}$}} & 26 & 13 & 11 & 7 & 4 & 3 & 28 & 92 \\
\hline & & 26 & 13 & 11 & 7 & 4 & 3 & 28 & 92 \\
\hline & & 28,3 & 14,1 & 12 & 7,6 & 4,3 & 3,3 & 30,4 & 100 \\
\hline & & 100 & 100 & 100 & 100 & 100 & 100 & 100 & 100 \\
\hline
\end{tabular}


Tabelle 3: Häufigkeitstabelle der Arztbesuche der Säuglinge

Arztbesuche der Säuglinge bis zum 4. Lebensmonat

\begin{tabular}{|c|r|r|}
\hline & Häufigkeit & Prozent \\
\hline 0 & 31 & 31,6 \\
1 & 41 & 41,8 \\
2 & 14 & 14,3 \\
3 & 4 & 4,1 \\
4 & 2 & 2,0 \\
5 & 3 & 3,1 \\
6 & 1 & 1,0 \\
7 & 1 & 1,0 \\
9 & 1 & 1,0 \\
GESAMT & 98 & 100 \\
Mittelwert & 1,29 & \\
Median & 1,0 & \\
Standardabweichung & 1,59 & \multicolumn{1}{|c}{} \\
\cline { 1 - 2 } & \multicolumn{2}{|c|}{}
\end{tabular}

Arztbesuche der Säuglinge vom 4. bis zum 8. Lebensmonat

\begin{tabular}{|l|r|r|}
\hline & Häufigkeit & Prozent \\
\hline \multicolumn{1}{|c|}{0} & 31 & 31,6 \\
1 & 36 & 36,7 \\
2 & 15 & 15,3 \\
3 & 6 & 6,1 \\
4 & 3 & 3,1 \\
7 & 1 & 1,0 \\
15 & 1 & 1,0 \\
Gültig Gesamt & 93 & 94,9 \\
Fehlend & 5 & 5,1 \\
GESAMT & 98 & 100 \\
Mittelwert & 1,27 & \\
Median & 1,0 & \\
Standardabweichung & 1,87 & \multicolumn{2}{|c}{} \\
\cline { 1 - 2 } & \multicolumn{2}{|c}{}
\end{tabular}


Arztbesuche der Säuglinge vom 8. bis zum 12. Lebensmonat

\begin{tabular}{|c|r|r|}
\hline & Häufigkeit & Prozent \\
\hline 0 & 25 & 25,5 \\
1 & 35 & 35,7 \\
2 & 16 & 16,3 \\
3 & 3 & 3,1 \\
4 & 3 & 3,1 \\
5 & 7 & 7,1 \\
6 & 1 & 1,0 \\
7 & 1 & 1,0 \\
9 & 1 & 1,0 \\
Gültig Gesamt & 92 & 93,9 \\
Fehlend & 6 & 6,1 \\
GESAMT & 98 & 100 \\
\cline { 2 - 3 } Mittelwert & 1,58 & \\
Median & 1,0 & \\
Standardabweichung & 1,77 & \\
\cline { 1 - 2 } & &
\end{tabular}


Tabelle 4: Zweigruppenvergleich (Chiquadrat-Tests) hinsichtlich der Arztbesuche

Arztbesuche der Säuglinge bis zum 4. Lebensmonat

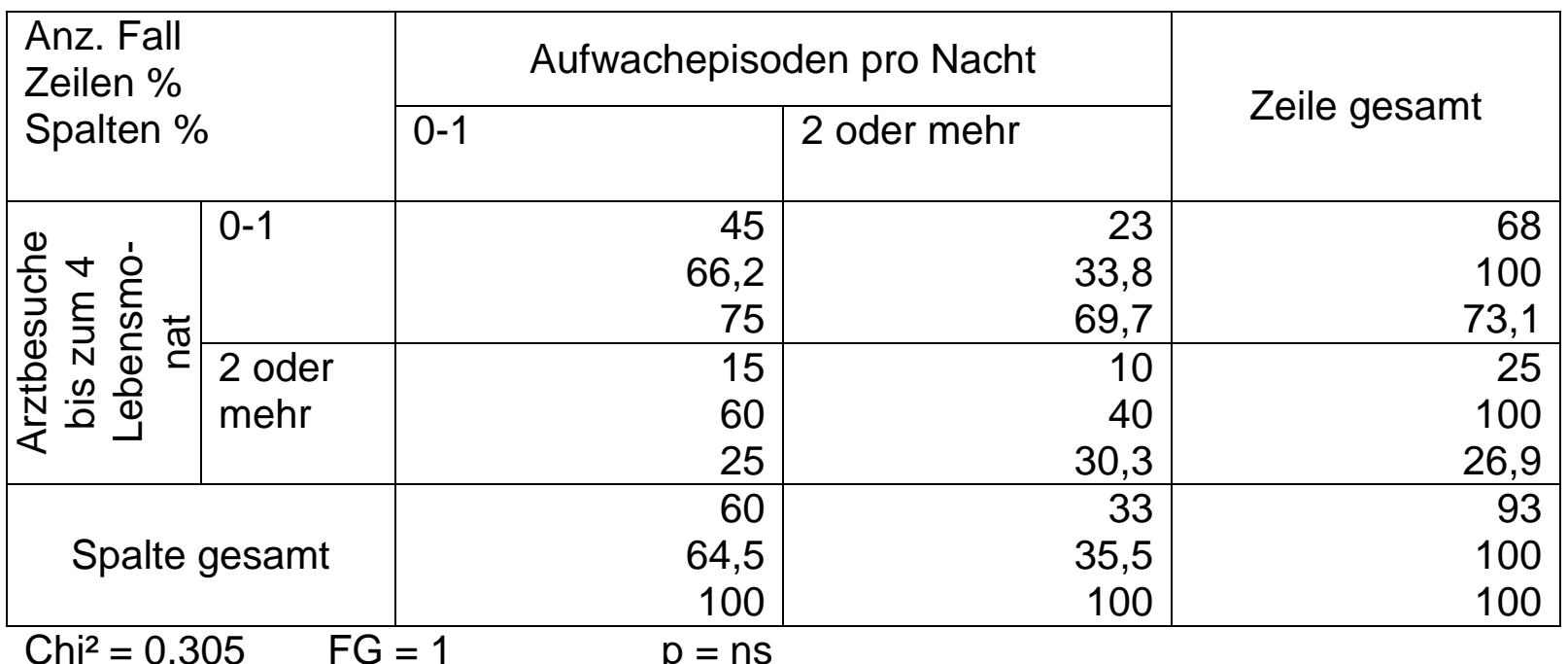

\begin{tabular}{|c|c|c|c|c|}
\hline \multirow{2}{*}{\multicolumn{2}{|c|}{$\begin{array}{l}\text { Anz. Fall } \\
\text { Zeilen \% } \\
\text { Spalten \% }\end{array}$}} & \multicolumn{2}{|c|}{ Nächte mit Aufwachepisoden pro Woche } & \multirow{2}{*}{ Zeile gesamt } \\
\hline & & $0-2$ & 3 oder mehr & \\
\hline \multirow{2}{*}{ 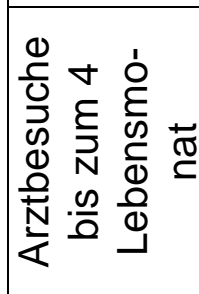 } & $0-1$ & $\begin{array}{r}40 \\
58,8 \\
80\end{array}$ & $\begin{array}{r}28 \\
41,2 \\
66,7\end{array}$ & $\begin{array}{r}68 \\
100 \\
73.9\end{array}$ \\
\hline & $\begin{array}{l}2 \text { oder } \\
\text { mehr }\end{array}$ & $\begin{array}{r}10 \\
41,7 \\
20\end{array}$ & $\begin{array}{r}14 \\
58,3 \\
33,3\end{array}$ & $\begin{array}{r}24 \\
100 \\
26,1\end{array}$ \\
\hline \multicolumn{2}{|c|}{ Spalte gesamt } & $\begin{array}{r}50 \\
54,3 \\
100\end{array}$ & $\begin{array}{r}42 \\
45,7 \\
100\end{array}$ & $\begin{array}{r}92 \\
100 \\
100\end{array}$ \\
\hline
\end{tabular}

$\mathrm{Chi}^{2}=2,105 \quad \mathrm{FG}=1 \quad \mathrm{p}=\mathrm{ns}$

Arztbesuche der Säuglinge vom 4. bis zum 8. Lebensmonat

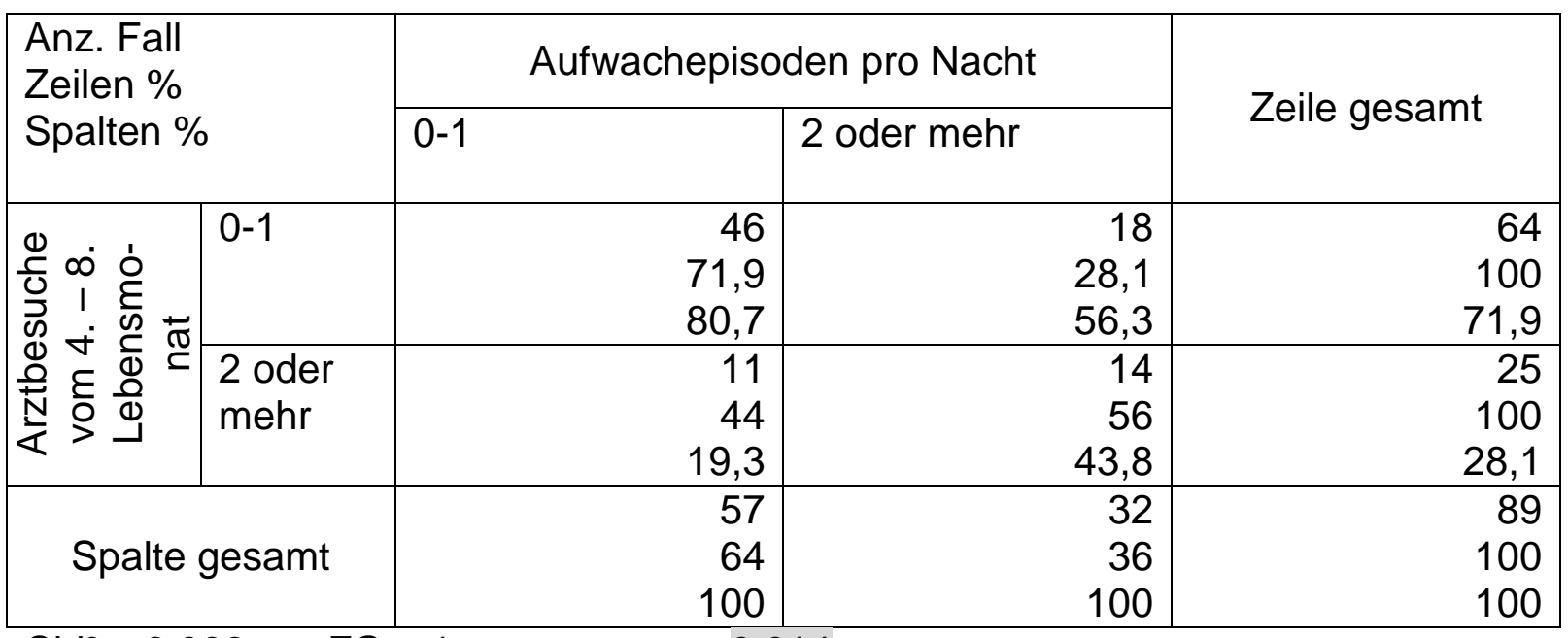

$\mathrm{Chi}^{2}=6,066 \quad \mathrm{FG}=1 \quad \mathrm{p}=0,014$ 


\begin{tabular}{|c|c|c|c|c|}
\hline \multirow{2}{*}{\multicolumn{2}{|c|}{$\begin{array}{l}\text { Anz. Fall } \\
\text { Zeilen \% } \\
\text { Spalten \% }\end{array}$}} & \multicolumn{2}{|c|}{ Nächte mit Aufwachepisoden pro Woche } & \multirow{2}{*}{ Zeile gesamt } \\
\hline & & $0-2$ & 3 oder mehr & \\
\hline 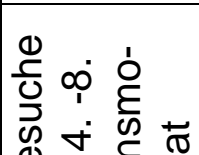 & $0-1$ & $\begin{array}{r}40 \\
63,5 \\
85,1 \\
\end{array}$ & $\begin{array}{r}23 \\
36,5 \\
56,1 \\
\end{array}$ & $\begin{array}{r}63 \\
100 \\
71,6 \\
\end{array}$ \\
\hline 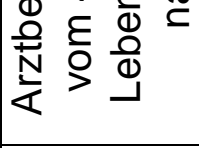 & $\begin{array}{l}2 \text { oder } \\
\text { mehr }\end{array}$ & $\begin{array}{r}7 \\
28 \\
14,9 \\
\end{array}$ & $\begin{array}{r}18 \\
72 \\
43,9 \\
\end{array}$ & $\begin{array}{r}25 \\
100 \\
28,4 \\
\end{array}$ \\
\hline \multicolumn{2}{|c|}{ Spalte gesamt } & $\begin{array}{r}47 \\
53,4 \\
100 \\
\end{array}$ & $\begin{array}{r}41 \\
46,6 \\
100 \\
\end{array}$ & $\begin{array}{r}88 \\
100 \\
100 \\
\end{array}$ \\
\hline
\end{tabular}

Arztbesuche der Säuglinge vom 8. bis zum 12. Lebensmonat

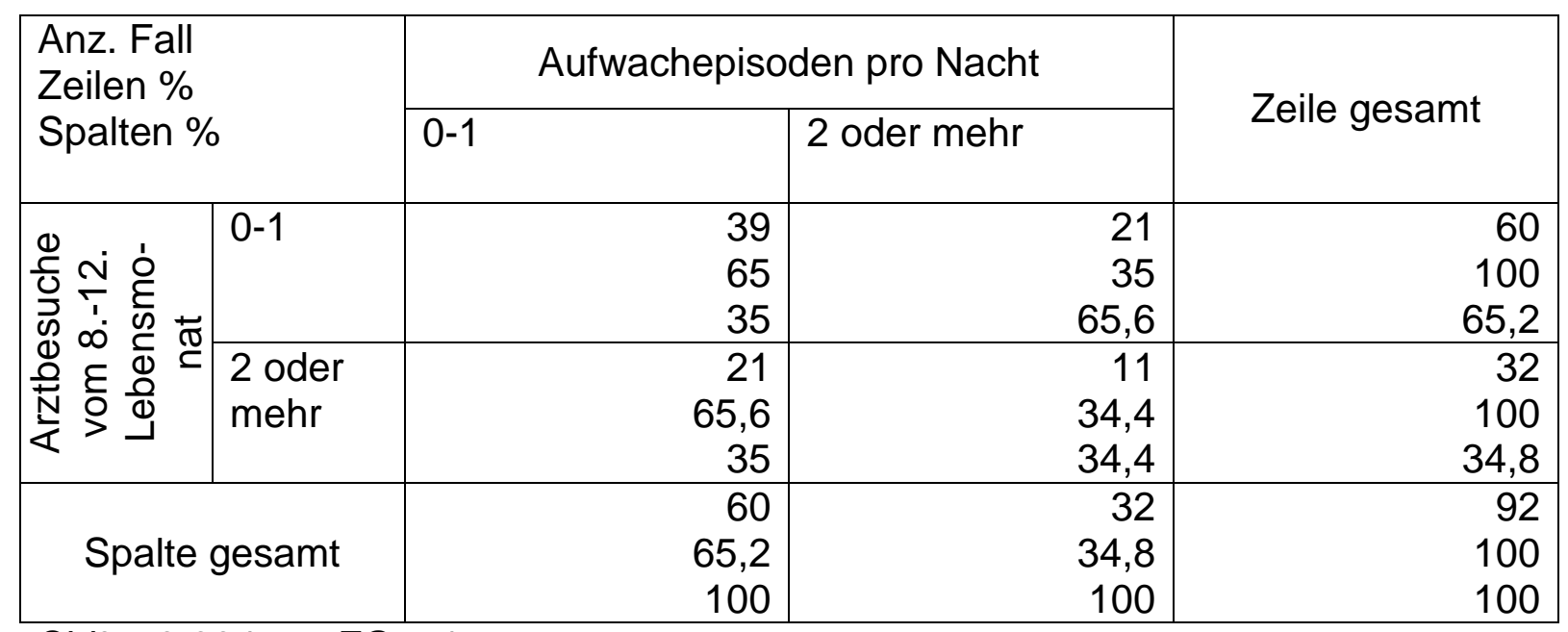

Chi $^{2}=0,004 \quad F G=1 \quad p=n s$

\begin{tabular}{|c|c|c|c|c|}
\hline \multirow{2}{*}{\multicolumn{2}{|c|}{$\begin{array}{l}\text { Anz. Fall } \\
\text { Zeilen \% } \\
\text { Spalten \% }\end{array}$}} & \multicolumn{2}{|c|}{ Nächte mit Aufwachepisoden pro Woche } & \multirow{2}{*}{ Zeile gesamt } \\
\hline & & $0-2$ & 3 oder mehr & \\
\hline 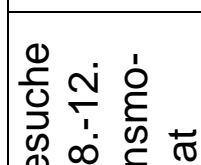 & $0-1$ & $\begin{array}{r}35 \\
59,3 \\
70 \\
\end{array}$ & $\begin{array}{r}24 \\
40,7 \\
58,5 \\
\end{array}$ & $\begin{array}{r}59 \\
100 \\
64,8 \\
\end{array}$ \\
\hline 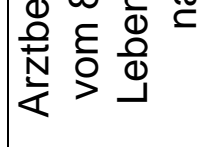 & $\begin{array}{l}2 \text { oder } \\
\text { mehr }\end{array}$ & $\begin{array}{r}15 \\
46,9 \\
30\end{array}$ & $\begin{array}{r}17 \\
53,1 \\
41,5\end{array}$ & $\begin{array}{r}32 \\
100 \\
35,2\end{array}$ \\
\hline \multicolumn{2}{|c|}{ Spalte gesamt } & $\begin{array}{r}50 \\
54,9 \\
100\end{array}$ & $\begin{array}{r}41 \\
45,1 \\
100\end{array}$ & $\begin{array}{r}91 \\
100 \\
100\end{array}$ \\
\hline
\end{tabular}


Tabelle 5: Häufigkeiten/ Statistiken zum Körpergewicht der Säuglinge

\begin{tabular}{|c|r|r|r|r|}
\hline & Geburtsgewicht & $\begin{array}{l}\text { Gewicht } \\
\text { bei U3 }\end{array}$ & $\begin{array}{l}\text { Gewicht } \\
\text { bei U5 }\end{array}$ & $\begin{array}{l}\text { Gewicht } \\
\text { bei U6 }\end{array}$ \\
\hline Gültige Anzahl & 98 & 97 & 94 & 90 \\
Fehlend & 0 & 1 & 4 & 8 \\
Mittelwert & 3414,64 & 4474,48 & 7974,31 & 9668,03 \\
Median & 3400 & 4480,00 & 7730,00 & 9535,00 \\
Standardabweichung & 441,15 & 616,91 & 1084,27 & 1196,42 \\
Minimum & 2500 & 3280 & 5590 & 7500 \\
Maximum & 4650 & 6420 & 11260 & 12700 \\
\hline
\end{tabular}

Tabelle 6: Pearson Rangkorrelationskoeffizienten des Körpergewichts und dem Schlafverhalten der Säuglinge

\begin{tabular}{|c|c|c|}
\hline & Aufwachen pro Nacht & $\begin{array}{l}\text { Nächte mit Wachepisoden pro } \\
\text { Woche }\end{array}$ \\
\hline Geburtsgewicht & $\begin{array}{l}r=-0,247 \\
p<0,017 \\
n=93\end{array}$ & $\begin{array}{l}r=-0,062 \\
p=n s \\
n=92\end{array}$ \\
\hline Gewicht bei U3 & $\begin{array}{l}r=-0,205 \\
p<0,049 \\
n=93\end{array}$ & $\begin{array}{l}r=-0,027 \\
p=n s \\
n=92\end{array}$ \\
\hline Gewicht bei U5 & $\begin{array}{l}r=-0,181 \\
p=n s \\
n=90\end{array}$ & $\begin{array}{l}r=0,024 \\
p=n s \\
n=89\end{array}$ \\
\hline Gewicht bei U6 & $\begin{array}{l}r=-0,215 \\
p<0,042 \\
n=90\end{array}$ & $\begin{array}{l}r=-0,088 \\
p=n s \\
n=89\end{array}$ \\
\hline
\end{tabular}

Anmerkung: Mitgeteilt sind punktbiseriale Korrelationskoeffizienten und Irrtumswahrscheinlichkeit (p); ns: nicht statistisch signifikant

Tabelle 7: Häufigkeiten/ Statistiken zum Stillverhalten

\begin{tabular}{|c|r|}
\hline & Stilldauer \\
\hline Gültige Anzahl & 91 \\
Fehlend & 7 \\
Mittelwert & 27,46 \\
Median & 26,00 \\
Standardabweichung & 13,49 \\
Minimum & 1 \\
Maximum & 48 \\
\hline
\end{tabular}


Tabelle 8: Pearson Rangkorrelationskoeffizienten des Stillverhaltens und dem Schlafverhalten der Säuglinge

\begin{tabular}{|c|c|c|}
\hline & $\begin{array}{c}\text { Aufwachepisoden } \\
\text { pro Nacht }\end{array}$ & $\begin{array}{l}\text { Nächte mit Wach- } \\
\text { episoden pro Wo- } \\
\text { che }\end{array}$ \\
\hline $\begin{array}{c}\text { Stilldauer } \\
\text { zum Zeitpunkt } \\
\text { des } 12 . \text { Mo- } \\
\text { nats }\end{array}$ & $\begin{array}{r}r=0,172 \\
n s \\
n=91\end{array}$ & $\begin{array}{r}r=0,040 \\
n s \\
n=90\end{array}$ \\
\hline
\end{tabular}

Tabelle 9: Spearman-Rho Rangkorrelationskoeffizienten des Stillverhaltens und dem Körpergewicht der Säuglinge

\begin{tabular}{|c|c|c|c|c|}
\hline & Geburtsgewicht & $\begin{array}{l}\text { Gewicht } \\
\text { bei U3 }\end{array}$ & $\begin{array}{l}\text { Gewicht } \\
\text { bei U5 }\end{array}$ & $\begin{array}{l}\text { Gewicht } \\
\text { bei U6 }\end{array}$ \\
\hline $\begin{array}{c}\text { Stilldauer } \\
\text { zum Zeitpunkt } \\
\text { des 8. Monats }\end{array}$ & $\begin{array}{r}r=0,147 \\
n s \\
n=94\end{array}$ & $\begin{array}{r}r=0,192 \\
n s \\
n=93\end{array}$ & $\begin{array}{r}r=-0,120 \\
n s \\
n=94\end{array}$ & $\begin{array}{r}r=-0,156 \\
n s \\
n=87\end{array}$ \\
\hline $\begin{array}{c}\text { Stilldauer } \\
\text { zum Zeitpunkt } \\
\text { des } 12 . \text { Mo- } \\
\text { nats }\end{array}$ & $\begin{array}{r}r=0,038 \\
n s \\
n=91\end{array}$ & $\begin{array}{r}r=0,211 \\
n s \\
n=91\end{array}$ & $\begin{array}{r}r=-0,029 \\
n s \\
n=88\end{array}$ & $\begin{array}{r}r=-0,107 \\
n s \\
n=88\end{array}$ \\
\hline
\end{tabular}

Tabelle 10: Pearson Rangkorrelationskoeffizienten der kognitiven Reife und dem Schlafverhalten der Säuglinge

\begin{tabular}{|c|c|c|}
\hline & $\begin{array}{c}\text { Aufwachepisoden } \\
\text { pro Nacht }\end{array}$ & $\begin{array}{l}\text { Nächte mit Wach- } \\
\text { episoden pro Wo- } \\
\text { che }\end{array}$ \\
\hline $\begin{array}{c}\text { Mental Develop- } \\
\text { mental Index } \\
\text { (MDI) im 4. Mo- } \\
\text { nat }\end{array}$ & $\begin{array}{r}r=0,058 \\
n s \\
n=88\end{array}$ & $\begin{array}{r}r=-0,024 \\
n s \\
n=88\end{array}$ \\
\hline $\begin{array}{l}\text { Mental Develop- } \\
\text { mental Index } \\
\text { (MDI) im } 8 . \\
\text { Monat }\end{array}$ & $\begin{array}{r}r=-0,195 \\
p=0,065 \\
n=90\end{array}$ & $\begin{array}{r}r=-0,058 \\
n s \\
n=89\end{array}$ \\
\hline $\begin{array}{l}\text { Mental Develop- } \\
\text { mental Index } \\
\text { (MDI) im } 12 . \\
\text { Monat }\end{array}$ & $\begin{array}{r}r=-0,013 \\
n s \\
n=93\end{array}$ & $\begin{array}{r}r=0,327 \\
p=0,001 \\
n=92\end{array}$ \\
\hline
\end{tabular}


Tabelle 11: Häufigkeitstabelle über Koliken der Säuglinge

Koliken von Geburt bis zum 4. Lebensmonat

\begin{tabular}{|l|r|r|}
\hline & Häufigkeit & Prozent \\
\hline \multicolumn{1}{|c|}{ Keine Koliken } & 48 & 49 \\
Etwas Koliken & 22 & 22,4 \\
Deutliche Koliken & 28 & 28,6 \\
Gültig Gesamt & 98 & 100 \\
Fehlend & 0 & 0 \\
GESAMT & 98 & 100 \\
& & \\
Mittelwert & 1,80 & \\
Median & 2,00 & \\
Standardabweichung & 0,86 & \\
Minimum & 1 & \\
Maximum & 3 &
\end{tabular}

$\underline{\text { Koliken im 4. Lebensmonat }}$

\begin{tabular}{|l|r|r|}
\hline & Häufigkeit & Prozent \\
\hline Keine Koliken & 81 & 82,7 \\
Etwas Koliken & 14 & 14,3 \\
Deutliche Koliken & 3 & 3,1 \\
Gültig Gesamt & 98 & 100 \\
Fehlend & 0 & 0 \\
GESAMT & 98 & 100 \\
& & \\
Mittelwert & 1,20 & \\
Median & 1,00 & \\
Standardabweichung & 0,48 & \\
Minimum & 1 & \\
Maximum & 3 &
\end{tabular}


Tabelle 12: Zweigruppenvergleich (Chiquadrat-Tests) hinsichtlich der Koliken

Koliken von Geburt bis zum 4. Lebensmonat

\begin{tabular}{|c|c|c|c|c|}
\hline \multirow{2}{*}{\multicolumn{2}{|c|}{$\begin{array}{l}\text { Anz. Fall } \\
\text { Erw. Wert } \\
\text { Zeilen \% } \\
\text { Spalten \% }\end{array}$}} & \multicolumn{2}{|c|}{ Aufwachepisoden pro Nacht } & \multirow{2}{*}{ Zeile gesamt } \\
\hline & & $0-1$ & 2 oder mehr & \\
\hline \multirow{6}{*}{ 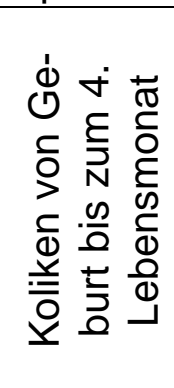 } & Keine & 43 & $\begin{array}{r}24 \\
238\end{array}$ & 67 \\
\hline & $\begin{array}{l}\text { bzw. et- } \\
\text { was }\end{array}$ & $\begin{array}{l}43,2 \\
64,2\end{array}$ & $\begin{array}{l}23,8 \\
35,8\end{array}$ & $\begin{array}{r}67 \\
100\end{array}$ \\
\hline & & 71,7 & 72,7 & 72 \\
\hline & Deutliche & $\begin{array}{r}17 \\
168\end{array}$ & 9 & 26 \\
\hline & & $\begin{array}{l}16,8 \\
65,4\end{array}$ & $\begin{array}{r}9,2 \\
34,6\end{array}$ & $\begin{array}{r}26 \\
100\end{array}$ \\
\hline & & 28,3 & 27,3 & 28 \\
\hline \multirow{4}{*}{\multicolumn{2}{|c|}{ Spalte gesamt }} & 60 & 33 & 93 \\
\hline & & 60 & 33 & 93 \\
\hline & & 64,5 & 35,5 & 100 \\
\hline & & 100 & 100 & 100 \\
\hline
\end{tabular}

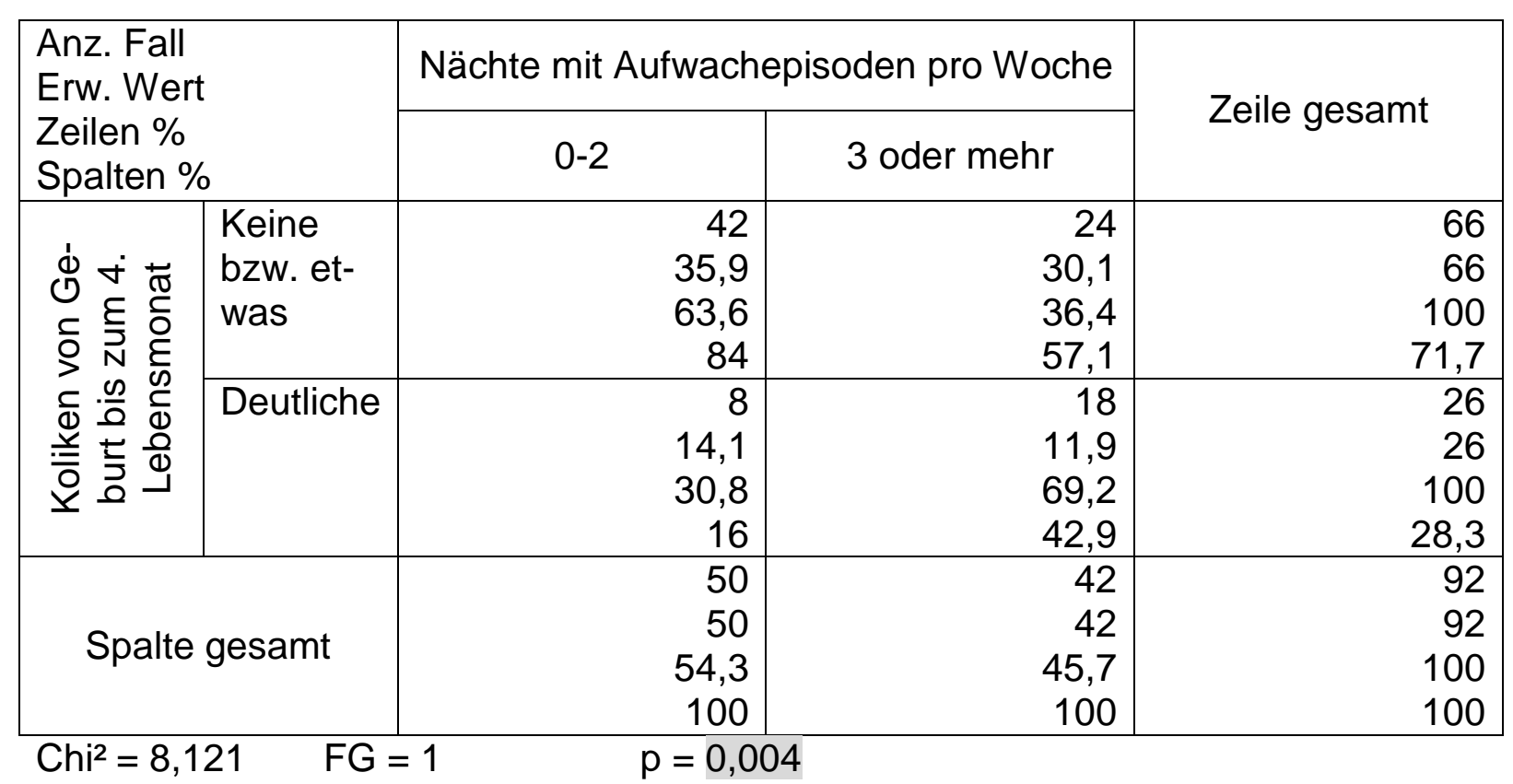


$\underline{\text { Koliken im 4. Lebensmonat }}$

\begin{tabular}{|c|c|c|c|c|}
\hline \multirow{2}{*}{\multicolumn{2}{|c|}{$\begin{array}{l}\text { Anz. Fall } \\
\text { Erw. Wert } \\
\text { Zeilen \% } \\
\text { Spalten \% }\end{array}$}} & \multicolumn{2}{|c|}{ Aufwachepisoden pro Nacht } & \multirow{2}{*}{ Zeile gesamt } \\
\hline & & $0-1$ & 2 oder mehr & \\
\hline \multirow{2}{*}{ 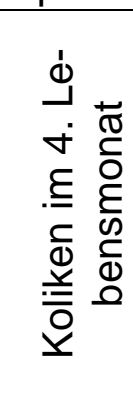 } & Keine & $\begin{array}{r}54 \\
50,3 \\
69,2 \\
90\end{array}$ & $\begin{array}{r}24 \\
27,7 \\
30,8 \\
72,7\end{array}$ & $\begin{array}{r}78 \\
78 \\
100 \\
83,9\end{array}$ \\
\hline & Etwas/deutliche & $\begin{array}{r}6 \\
9,7 \\
40 \\
10\end{array}$ & $\begin{array}{r}9 \\
5,3 \\
60 \\
27,3 \\
\end{array}$ & $\begin{array}{r}15 \\
15 \\
100 \\
16,1\end{array}$ \\
\hline \multicolumn{2}{|c|}{ Spalte gesamt } & $\begin{array}{r}60 \\
60 \\
64,5 \\
100\end{array}$ & $\begin{array}{r}33 \\
33 \\
35,5 \\
100\end{array}$ & $\begin{array}{r}93 \\
93 \\
100 \\
100\end{array}$ \\
\hline
\end{tabular}

\begin{tabular}{|c|c|c|c|c|}
\hline \multirow{2}{*}{\multicolumn{2}{|c|}{$\begin{array}{l}\text { Anz. Fall } \\
\text { Erw. Wert } \\
\text { Zeilen \% } \\
\text { Spalten \% }\end{array}$}} & \multicolumn{2}{|c|}{$\begin{array}{l}\text { Nächte mit Aufwachepisoden pro } \\
\text { Woche }\end{array}$} & \multirow{2}{*}{ Zeile gesamt } \\
\hline & & $0-2$ & 3 oder mehr & \\
\hline \multirow{8}{*}{ 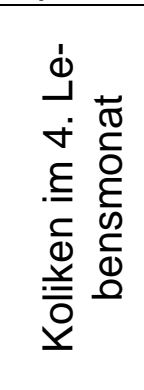 } & Keine & 44 & 33 & 77 \\
\hline & & 41,8 & 35,2 & 77 \\
\hline & & 57,1 & 42,9 & 100 \\
\hline & & 88 & 78,6 & 83,7 \\
\hline & Etwas/deutliche & 6 & 9 & 15 \\
\hline & & 8,2 & 6,8 & 15 \\
\hline & & 40 & 60 & 100 \\
\hline & & 12 & 21,4 & 16,3 \\
\hline \multirow{4}{*}{\multicolumn{2}{|c|}{ Spalte gesamt }} & 50 & 42 & 92 \\
\hline & & 50 & 42 & 92 \\
\hline & & 54,3 & 45,7 & 100 \\
\hline & & 100 & 100 & 100 \\
\hline
\end{tabular}


Tabelle 13: Pearson Rangkorrelationskoeffizienten der positiven Emotionalität und dem Schlafverhalten der Säuglinge

\begin{tabular}{|c|c|c|c|}
\hline & \multicolumn{3}{|c|}{ Aufwachepisoden pro Nacht } \\
\hline & 4 Monate & 8 Monate & 12 Monate \\
\hline $\begin{array}{c}\text { Fragebogenskala } \\
\text { „Lachen / Lä- } \\
\text { cheln“" }\end{array}$ & $\begin{array}{r}r=-0,299 \\
p<0,004 \\
n=93\end{array}$ & $\begin{array}{r}r=-0,201 \\
p<0,059 \\
n=89\end{array}$ & $\begin{array}{r}r=-0,287 \\
p<0,006 \\
n=92\end{array}$ \\
\hline $\begin{array}{c}\text { Verhaltenstest } \\
\text { „positive Emotio- } \\
\text { nalität“، }\end{array}$ & $\begin{array}{r}r=-0,063 \\
n s \\
n=88\end{array}$ & $\begin{array}{r}r=-0,232 \\
p<0,029 \\
n=89\end{array}$ & $\begin{array}{r}r=-0,221 \\
p<0,033 \\
n=93\end{array}$ \\
\hline
\end{tabular}

Anmerkung: *Mitgeteilt sind Phi-Korrelationskoeffizienten und Irrtumswahrscheinlichkeit (p); ns: nicht statistisch signifikant. ${ }^{* *}$ Mitgeteilt sind punktbiseriale Korrelationskoeffizienten und Irrtumswahrscheinlichkeit (p); ns: nicht statistisch signifikant.

\begin{tabular}{|c|c|c|c|}
\hline & \multicolumn{3}{|c|}{ Nächte mit Aufwachepisoden pro Woche } \\
\hline & 4 Monate & 8 Monate & 12 Monate \\
\hline Fragebogenskala & $r=-0,165$ & $r=-0,118$ & $r=-0,080$ \\
\hline $\begin{array}{l}\text { „Lachen / Lä- } \\
\text { cheln“ }\end{array}$ & $\mathrm{n}=92$ & $\begin{array}{r}n s \\
n=88\end{array}$ & $\begin{array}{r}\mathrm{ns} \\
\mathrm{n}=91\end{array}$ \\
\hline Verhaltenstest & $r=-0,013$ & $r=0,073$ & $r=0,083$ \\
\hline $\begin{array}{c}\text { „positive Emotio- } \\
\text { nalität““ }\end{array}$ & $\begin{array}{r}\mathrm{ns} \\
\mathrm{n}=88\end{array}$ & $\begin{array}{r}\mathrm{ns} \\
\mathrm{n}=88\end{array}$ & $\begin{array}{r}\mathrm{ns} \\
\mathrm{n}=92\end{array}$ \\
\hline
\end{tabular}

Anmerkung: *Mitgeteilt sind Phi-Korrelationskoeffizienten und Irrtumswahrscheinlichkeit (p); ns: nicht statistisch signifikant. ${ }^{* *}$ Mitgeteilt sind punktbiseriale Korrelationskoeffizienten und

Irrtumswahrscheinlichkeit (p); ns: nicht statistisch signifikant. 
Tabelle 14: Pearson Rangkorrelationskoeffizienten der negativen Emotionalität und dem Schlafverhalten der Säuglinge

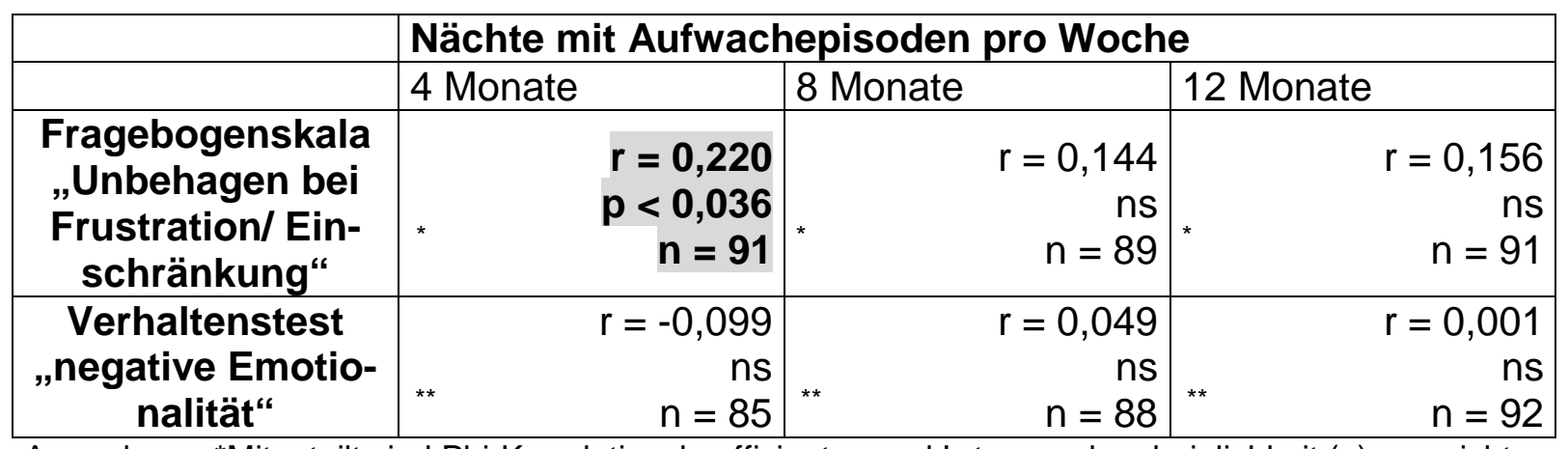

Anmerkung: *Mitgeteilt sind Phi-Korrelationskoeffizienten und Irrtumswahrscheinlichkeit (p); ns: nicht statistisch signifikant. ${ }^{* *}$ Mitgeteilt sind punktbiseriale Korrelationskoeffizienten und

Irrtumswahrscheinlichkeit (p); ns: nicht statistisch signifikant.

\begin{tabular}{|c|c|c|c|c|}
\hline & \multicolumn{4}{|c|}{ Aufwachepisoden pro Nacht } \\
\hline & 4 Monate & 8 Monate & & 12 Monate \\
\hline $\begin{array}{c}\text { Fragebogenskala } \\
\text { „Unbehagen bei } \\
\text { Frustration/ Ein- } \\
\text { schränkung }\end{array}$ & $\begin{array}{r}r=0,079 \\
n s \\
n=92\end{array}$ & $\begin{array}{r}r=0,06 \\
n \\
n=9\end{array}$ & & $\begin{array}{r}r=0,155 \\
n s \\
n=92\end{array}$ \\
\hline $\begin{array}{c}\text { Verhaltenstest } \\
\text { „,negative Emotio- } \\
\text { nalität“‘ }\end{array}$ & $\begin{array}{r}r=-0,047 \\
n s \\
n=85\end{array}$ & $\begin{array}{r}r=0,13 \\
n \\
n=8\end{array}$ & & $\begin{array}{r}r=0,259 \\
p<.012 \\
n=93\end{array}$ \\
\hline
\end{tabular}

Anmerkung: *Mitgeteilt sind Phi-Korrelationskoeffizienten und Irrtumswahrscheinlichkeit (p); ns: nicht statistisch signifikant. ${ }^{* *}$ Mitgeteilt sind punktbiseriale Korrelationskoeffizienten und Irrtumswahrscheinlichkeit (p); ns: nicht statistisch signifikant. 
Tabelle 15: Zweigruppenvergleich (Chiquadrat-Tests) hinsichtlich der positiven Emotionalität

$\underline{\text { Positive Emotionalität im Alter von } 4 \text { Monaten }}$

\begin{tabular}{|c|c|c|c|c|}
\hline \multirow{2}{*}{\multicolumn{2}{|c|}{$\begin{array}{l}\text { Anz. Fall } \\
\text { Erw. Wert } \\
\text { Zeilen \% } \\
\text { Spalten \% }\end{array}$}} & \multicolumn{2}{|c|}{ Aufwachepisoden pro Nacht } & \multirow{2}{*}{ Zeile gesamt } \\
\hline & & $0-1$ & 2 oder mehr & \\
\hline \multirow{2}{*}{ 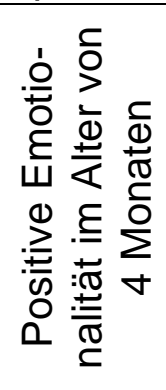 } & gering & $\begin{array}{r}39 \\
40,2 \\
63,9 \\
67,2\end{array}$ & $\begin{array}{r}22 \\
20,8 \\
36,1 \\
73,3 \\
\end{array}$ & $\begin{array}{r}61 \\
61 \\
100 \\
69,3 \\
\end{array}$ \\
\hline & deutlich & $\begin{array}{r}19 \\
17,8 \\
70,4 \\
32,8\end{array}$ & $\begin{array}{r}8 \\
9,2 \\
29,6 \\
26,7\end{array}$ & $\begin{array}{r}27 \\
27 \\
100 \\
30,7\end{array}$ \\
\hline \multicolumn{2}{|c|}{ Spalte gesamt } & $\begin{array}{r}58 \\
58 \\
65,9 \\
100\end{array}$ & $\begin{array}{r}30 \\
30 \\
34,1 \\
100\end{array}$ & $\begin{array}{r}88 \\
88 \\
100 \\
100\end{array}$ \\
\hline
\end{tabular}

\begin{tabular}{|c|c|c|c|c|}
\hline \multirow{2}{*}{\multicolumn{2}{|c|}{$\begin{array}{l}\text { Anz. Fall } \\
\text { Erw. Wert } \\
\text { Zeilen \% } \\
\text { Spalten \% }\end{array}$}} & \multicolumn{2}{|c|}{ Nächte mit Aufwachepisoden pro Woche } & \multirow{2}{*}{ Zeile gesamt } \\
\hline & & $0-2$ & 3 oder mehr & \\
\hline \multirow{2}{*}{ 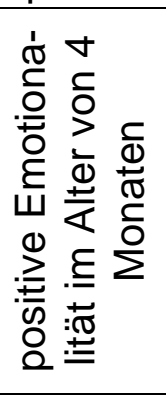 } & gering & $\begin{array}{r}33 \\
33,3 \\
54,1 \\
68,8\end{array}$ & $\begin{array}{r}28 \\
27,7 \\
45,9 \\
70\end{array}$ & $\begin{array}{r}61 \\
61 \\
100 \\
69,3\end{array}$ \\
\hline & deutlich & $\begin{array}{r}15 \\
14,7 \\
55,6 \\
31,3\end{array}$ & $\begin{array}{r}12 \\
12,3 \\
44,4 \\
30\end{array}$ & $\begin{array}{r}27 \\
27 \\
100 \\
30,7\end{array}$ \\
\hline \multicolumn{2}{|c|}{ Spalte gesamt } & $\begin{array}{r}48 \\
48 \\
54,5 \\
100\end{array}$ & $\begin{array}{r}40 \\
40 \\
45,5 \\
100 \\
\end{array}$ & $\begin{array}{r}88 \\
88 \\
100 \\
100 \\
\end{array}$ \\
\hline
\end{tabular}


positive Emotionalität im Alter von 8 Monaten

\begin{tabular}{|c|c|c|c|c|}
\hline \multirow{2}{*}{\multicolumn{2}{|c|}{\begin{tabular}{|l|} 
Anz. Fall \\
Erw. Wert \\
Zeilen \% \\
Spalten \% \\
\end{tabular}}} & \multicolumn{2}{|c|}{ Aufwachepisoden pro Nacht } & \multirow{2}{*}{ Zeile gesamt } \\
\hline & & $0-1$ & 2 oder mehr & \\
\hline \multirow{7}{*}{ 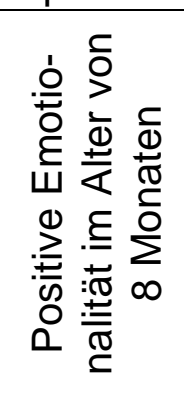 } & gering & 25 & 18 & 43 \\
\hline & & $\begin{array}{l}27,1 \\
58.1\end{array}$ & $\begin{array}{l}15,9 \\
41.9\end{array}$ & $\begin{array}{r}43 \\
100\end{array}$ \\
\hline & & 44,6 & 54,5 & 48,3 \\
\hline & deutlich & 31 & 15 & 46 \\
\hline & & 28,9 & 17,1 & 46 \\
\hline & & 67,4 & 32,6 & 100 \\
\hline & & 55,4 & 45,5 & 51,7 \\
\hline \multirow{4}{*}{\multicolumn{2}{|c|}{ Spalte gesamt }} & 56 & 33 & 89 \\
\hline & & 56 & 33 & 89 \\
\hline & & 62,9 & 37,1 & 100 \\
\hline & & 100 & 100 & 100 \\
\hline
\end{tabular}

\begin{tabular}{|c|c|c|c|c|}
\hline \multirow{2}{*}{\multicolumn{2}{|c|}{$\begin{array}{l}\text { Anz. Fall } \\
\text { Erw. Wert } \\
\text { Zeilen \% } \\
\text { Spalten \% }\end{array}$}} & \multicolumn{2}{|c|}{ Nächte mit Aufwachepisoden pro Woche } & \multirow{2}{*}{ Zeile gesamt } \\
\hline & & $0-2$ & 3 oder mehr & \\
\hline \multirow{2}{*}{ 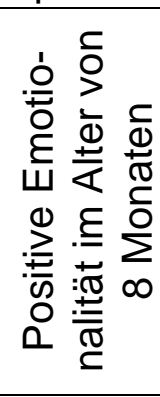 } & gering & $\begin{array}{r}24 \\
23 \\
55,8 \\
51,1\end{array}$ & $\begin{array}{r}19 \\
20 \\
44,2 \\
46,3\end{array}$ & $\begin{array}{r}43 \\
43 \\
100 \\
48,9\end{array}$ \\
\hline & deutlich & $\begin{array}{r}23 \\
24 \\
51,1 \\
48,9\end{array}$ & $\begin{array}{r}22 \\
21 \\
48,9 \\
53,7\end{array}$ & $\begin{array}{r}45 \\
45 \\
100 \\
51,1\end{array}$ \\
\hline \multicolumn{2}{|c|}{ Spalte gesamt } & $\begin{array}{r}47 \\
47 \\
53,4 \\
100\end{array}$ & $\begin{array}{r}41 \\
41 \\
46,6 \\
100\end{array}$ & $\begin{array}{r}88 \\
88 \\
100 \\
100\end{array}$ \\
\hline
\end{tabular}


positive Emotionalität im Alter von 12 Monaten

\begin{tabular}{|c|c|c|c|c|}
\hline \multirow{2}{*}{\multicolumn{2}{|c|}{$\begin{array}{l}\text { Anz. Fall } \\
\text { Erw. Wert } \\
\text { Zeilen \% } \\
\text { Spalten \% }\end{array}$}} & \multicolumn{2}{|c|}{ Aufwachepisoden pro Nacht } & \multirow{2}{*}{ Zeile gesamt } \\
\hline & & $0-1$ & 2 oder mehr & \\
\hline \multirow{2}{*}{ 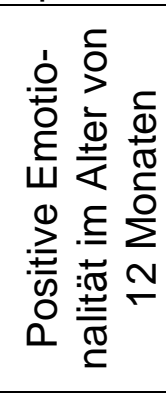 } & gering & $\begin{array}{r}25 \\
29,7 \\
54,3 \\
41,7\end{array}$ & $\begin{array}{r}21 \\
16,3 \\
45,7 \\
63,6\end{array}$ & $\begin{array}{r}46 \\
46 \\
100 \\
49,5\end{array}$ \\
\hline & deutlich & $\begin{array}{r}35 \\
30,3 \\
74,5 \\
58,3\end{array}$ & $\begin{array}{r}12 \\
16,7 \\
25,5 \\
36,4 \\
\end{array}$ & $\begin{array}{r}47 \\
47 \\
100 \\
50,5\end{array}$ \\
\hline \multicolumn{2}{|c|}{ Spalte gesamt } & $\begin{array}{r}60 \\
60 \\
64,5 \\
100\end{array}$ & $\begin{array}{r}33 \\
33 \\
35,5 \\
100\end{array}$ & $\begin{array}{r}93 \\
93 \\
100 \\
100\end{array}$ \\
\hline
\end{tabular}

\begin{tabular}{|c|c|c|c|c|}
\hline \multirow{2}{*}{\multicolumn{2}{|c|}{$\begin{array}{l}\text { Anz. Fall } \\
\text { Erw. Wert } \\
\text { Zeilen \% } \\
\text { Spalten \% }\end{array}$}} & \multicolumn{2}{|c|}{ Nächte mit Aufwachepisoden pro Woche } & \multirow{2}{*}{ Zeile gesamt } \\
\hline & & $0-2$ & 3 oder mehr & \\
\hline \multirow{2}{*}{ 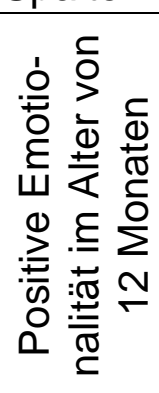 } & gering & $\begin{array}{r}26 \\
25 \\
56,5 \\
52\end{array}$ & $\begin{array}{r}20 \\
21 \\
43,5 \\
47,6\end{array}$ & $\begin{array}{r}46 \\
46 \\
100 \\
50\end{array}$ \\
\hline & deutlich & $\begin{array}{r}24 \\
25 \\
52,2 \\
48\end{array}$ & $\begin{array}{r}22 \\
21 \\
47,8 \\
52,4\end{array}$ & $\begin{array}{r}46 \\
46 \\
100 \\
50\end{array}$ \\
\hline \multicolumn{2}{|c|}{ Spalte gesamt } & $\begin{array}{r}50 \\
50 \\
54,3 \\
100\end{array}$ & $\begin{array}{r}42 \\
42 \\
45,7 \\
100\end{array}$ & $\begin{array}{r}92 \\
92 \\
100 \\
100\end{array}$ \\
\hline
\end{tabular}


Tabelle 16: Zweigruppenvergleich (Chiquadrat-Tests) hinsichtlich der negativen Emotionalität

$\underline{\text { negative Emotionalität im Alter von } 4 \text { Monaten }}$

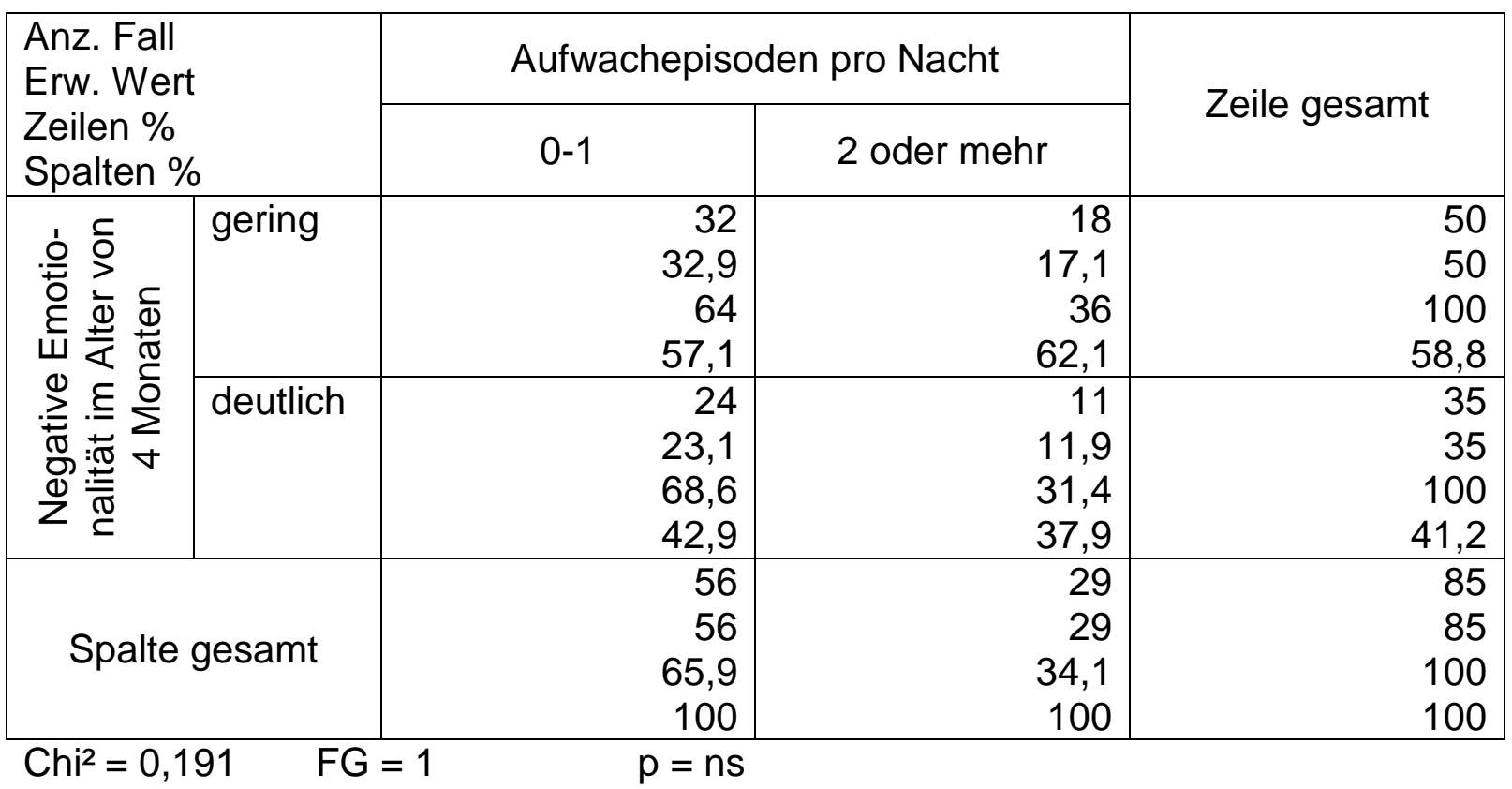

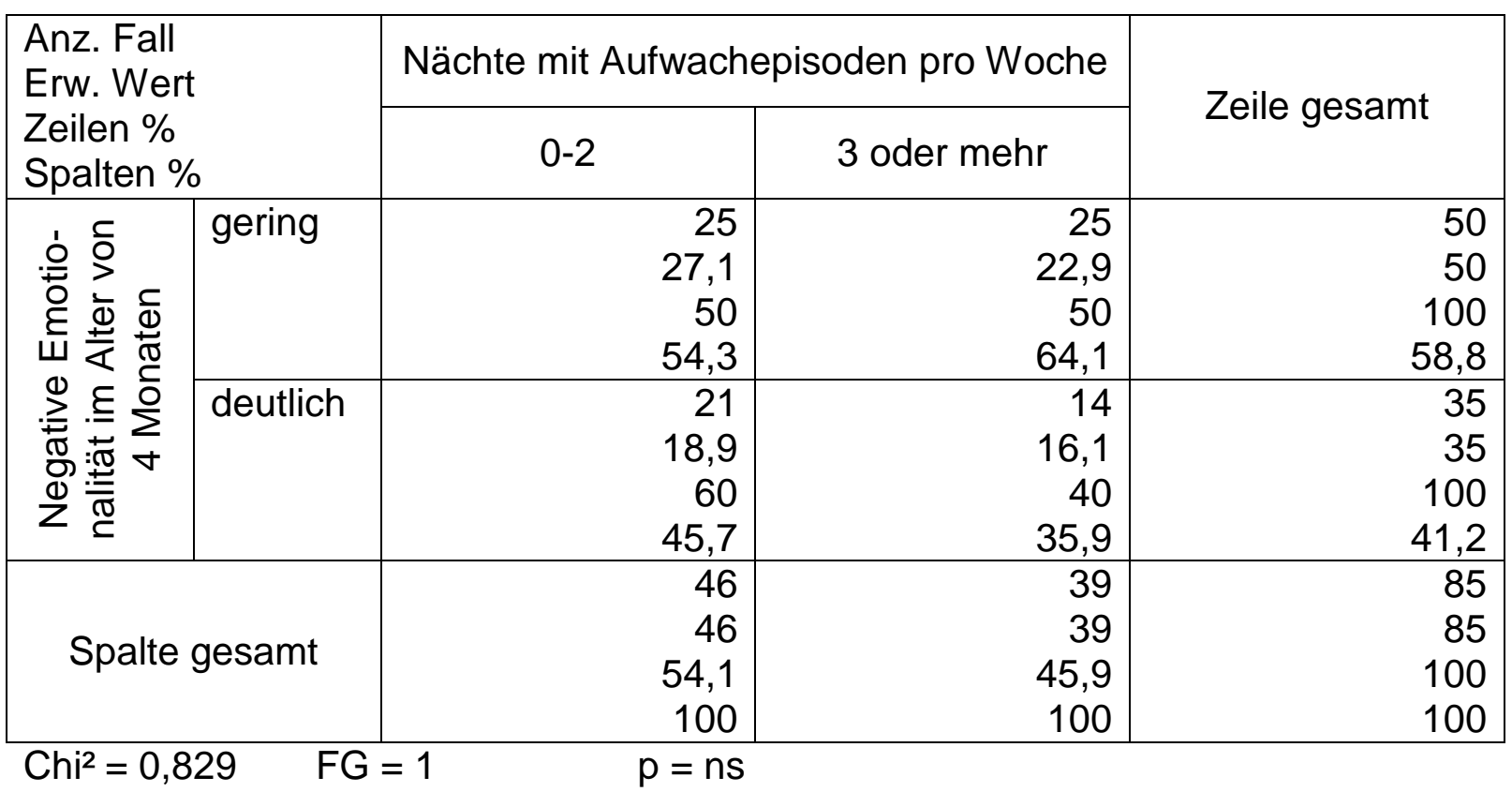


$\underline{\text { negative Emotionalität im Alter von } 8 \text { Monaten }}$

\begin{tabular}{|c|c|c|c|c|}
\hline \multirow{2}{*}{\multicolumn{2}{|c|}{$\begin{array}{l}\text { Anz. Fall } \\
\text { Erw. Wert } \\
\text { Zeilen \% } \\
\text { Spalten \% }\end{array}$}} & \multicolumn{2}{|c|}{ Aufwachepisoden pro Nacht } & \multirow{2}{*}{ Zeile gesamt } \\
\hline & & $0-1$ & 2 oder mehr & \\
\hline \multirow{7}{*}{ 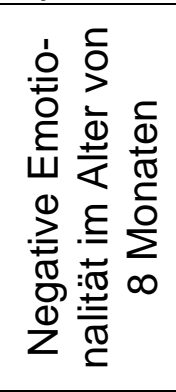 } & gering & 39 & 18 & 57 \\
\hline & & $\begin{array}{l}35,9 \\
68,4\end{array}$ & $\begin{array}{l}21,1 \\
31,6\end{array}$ & $\begin{array}{r}5 / \\
100\end{array}$ \\
\hline & & 69,6 & 54,5 & 64 \\
\hline & deutlich & 17 & 15 & 32 \\
\hline & & 20,1 & 11,9 & 32 \\
\hline & & 53,1 & 46,9 & 100 \\
\hline & & 30,4 & 45,5 & 36 \\
\hline \multirow{4}{*}{\multicolumn{2}{|c|}{ Spalte gesamt }} & 56 & 33 & 89 \\
\hline & & 56 & 33 & 89 \\
\hline & & 62,9 & 37,1 & 100 \\
\hline & & 100 & 100 & 100 \\
\hline
\end{tabular}

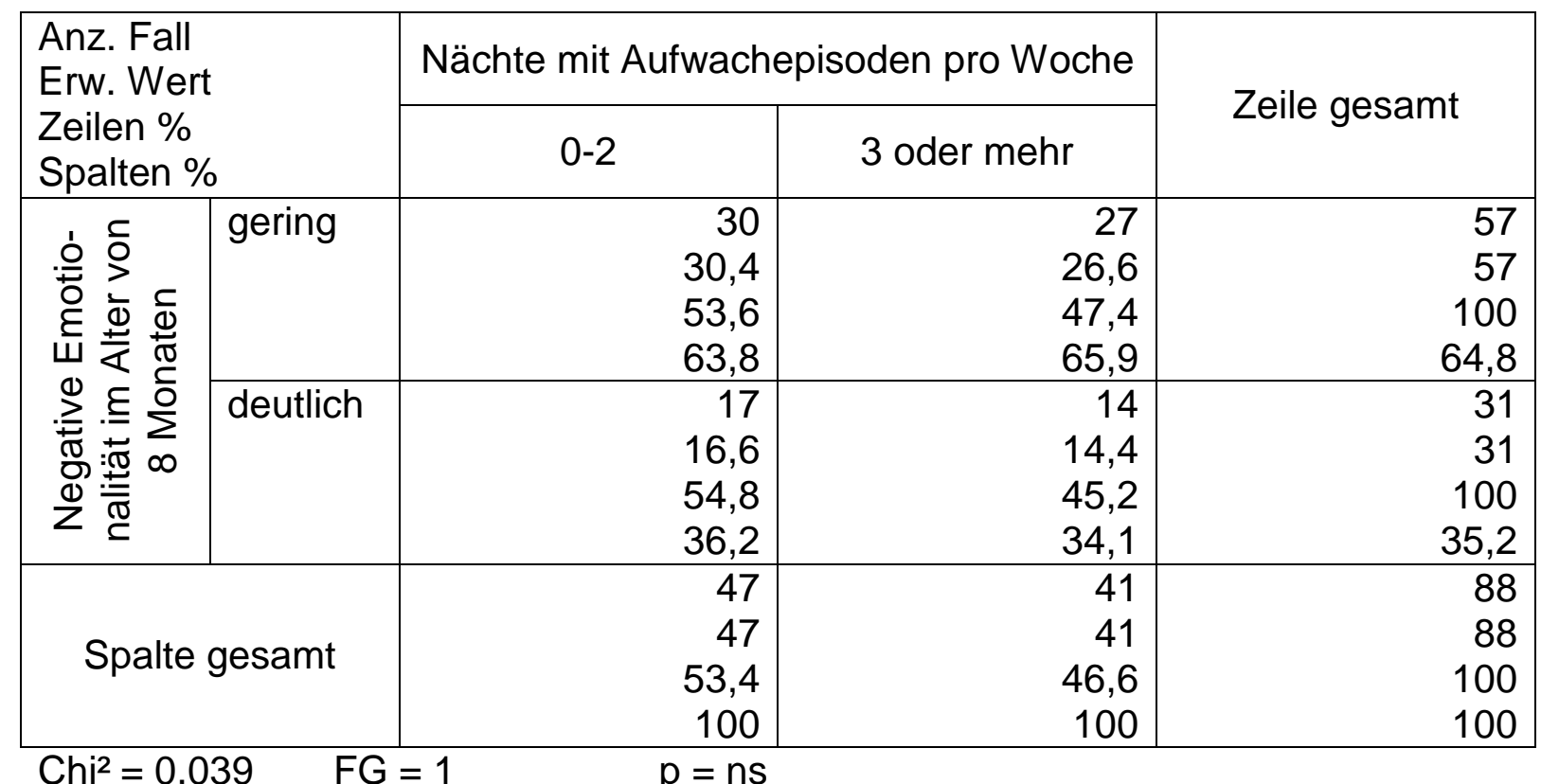


$\underline{\text { negative Emotionalität im Alter von } 12 \text { Monaten }}$

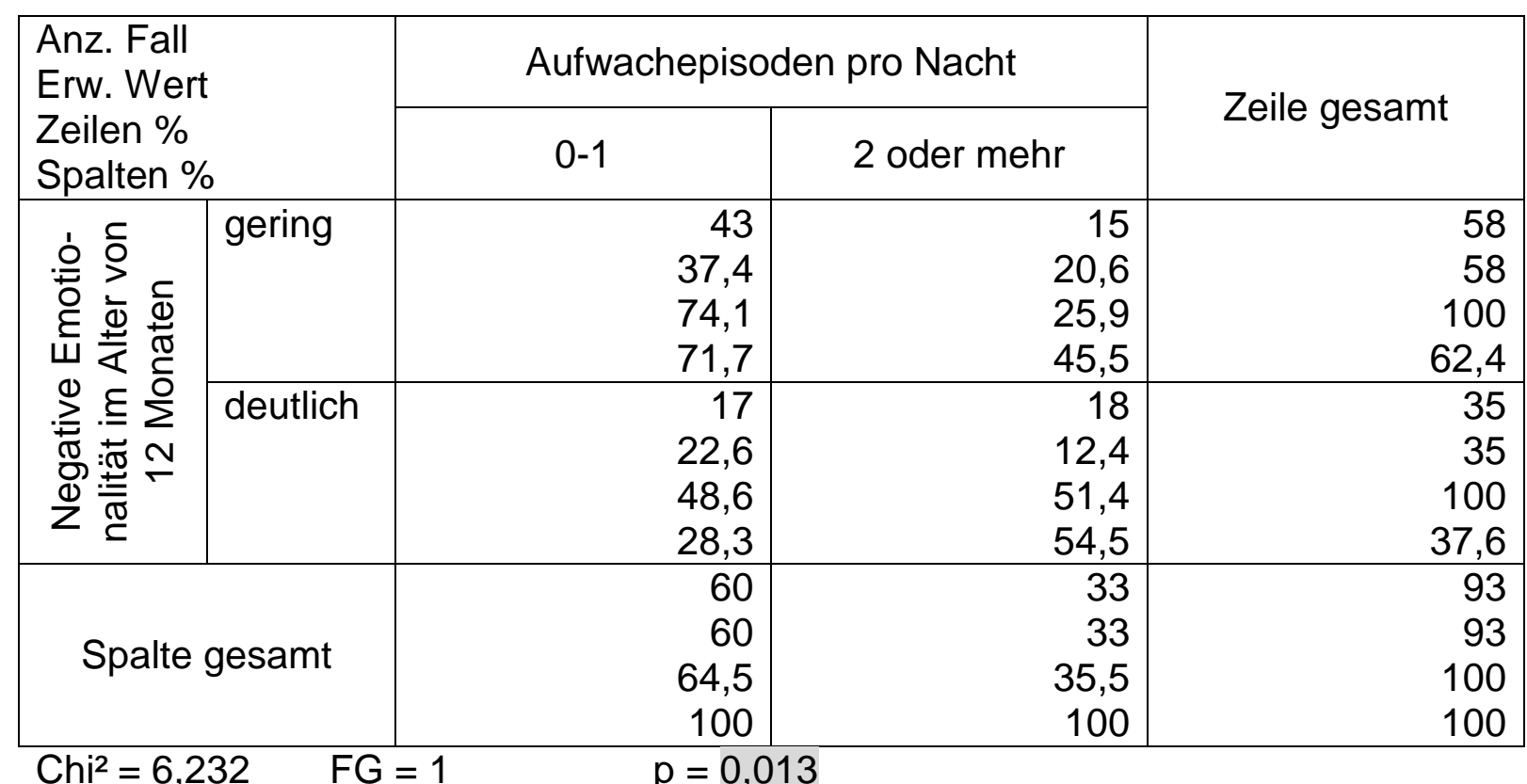

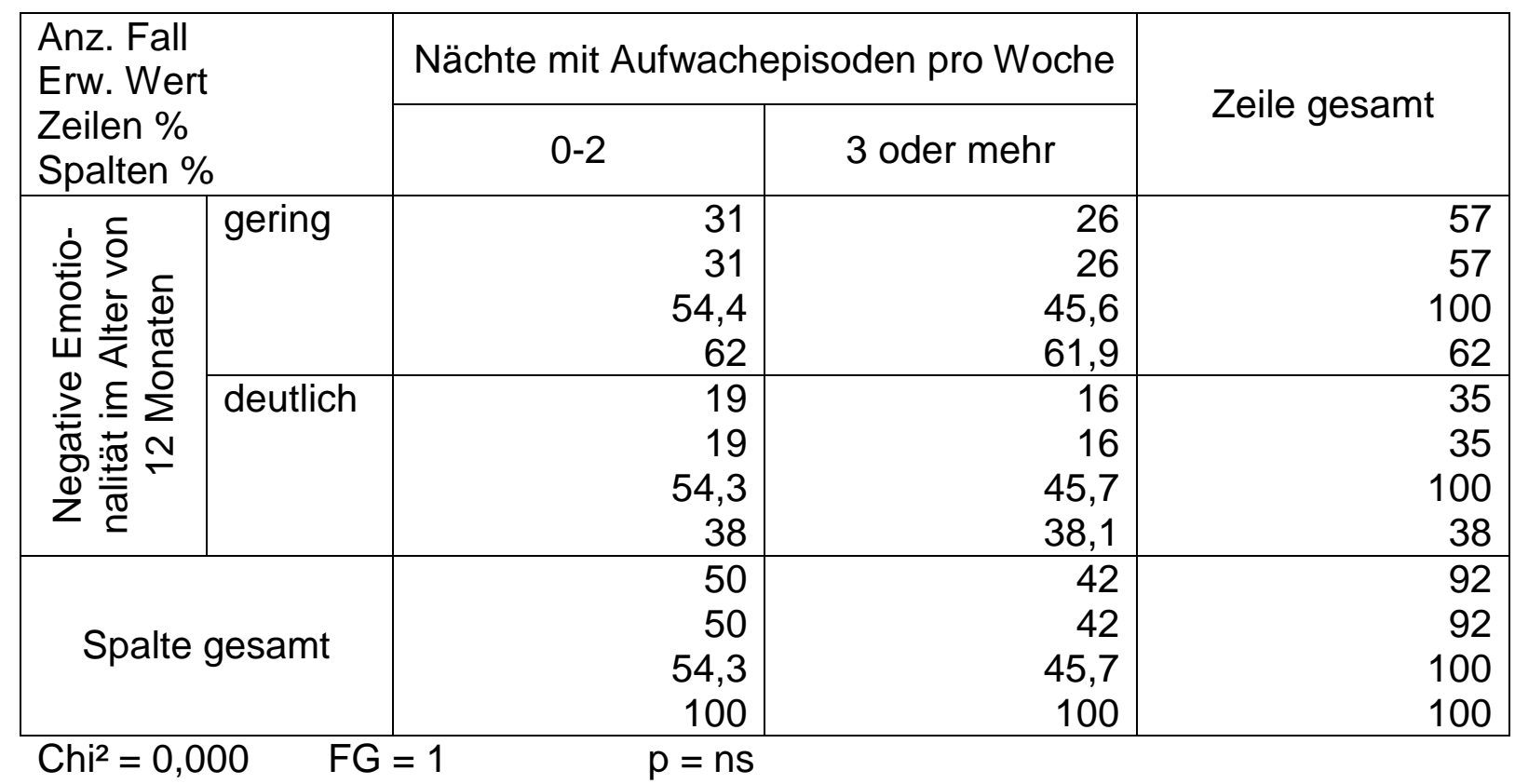


Tabelle 17: Pearson Rangkorrelationskoeffizienten der mütterlichen Merkmale und dem Schlafverhalten der Säuglinge

\begin{tabular}{|c|r|rr|r|}
\hline & \multicolumn{3}{|l|}{ Aufwachepisoden pro Nacht } \\
\hline & 4 Monate & 8 Monate & 12 Monate \\
\hline Fragebogenskala & $\mathbf{r}=\mathbf{0 , 2 3 1}$ & $\mathrm{r}=0,139$ & $\mathbf{r}=\mathbf{0 , 2 2 3}$ \\
"Depressivität“" & $\mathbf{p}<. \mathbf{0 2 9}$ & $\mathrm{ns}$ & $\mathbf{p}<. \mathbf{0 4 6}$ \\
& $\mathbf{n = 8 9}$ & $\mathrm{n}=83$ & $\mathbf{n = 8 1}$ \\
\hline
\end{tabular}

Anmerkung: Mitgeteilt sind punktbiseriale Korrelationskoeffizienten und Irrtumswahrscheinlichkeit (p); ns: nicht statistisch signifikant.

\begin{tabular}{|c|r|r|r|}
\hline & \multicolumn{3}{|l|}{ Nächte mit Aufwachepisoden pro Woche } \\
\hline & 4 Monate & 8 Monate & 12 Monate \\
\hline Fragebogenskala & $r=-0,159$ & $r=-0,010$ & $r=-0,001$ \\
„Depressivität“ & $n s$ & $n s$ & $n s$ \\
& $n=88$ & $n=82$ & $n=80$ \\
\hline
\end{tabular}

Anmerkung: Mitgeteilt sind punktbiseriale Korrelationskoeffizienten und Irrtumswahrscheinlichkeit (p); ns: nicht statistisch signifikant.

\begin{tabular}{|c|r|rr|r|}
\hline & \multicolumn{3}{|l|}{ Aufwachepisoden pro Nacht } \\
\hline & 4 Monate & 8 Monate & 12 Monate \\
\hline $\begin{array}{c}\text { Fragebogenskala } \\
\text { „Hoffnungslosig- }\end{array}$ & $\mathbf{r}=\mathbf{0 , 2 0 9}$ & $\mathrm{r}=0,040$ & $\mathrm{r}=0,099$ \\
keit““ & $\mathbf{p}<.044$ & $\mathrm{~ns}$ & $\mathrm{~ns}$ \\
$\mathbf{n = 9 3}$ & $\mathrm{n}=88$ & $\mathrm{n}=84$ \\
\hline
\end{tabular}

Anmerkung: Mitgeteilt sind punktbiseriale Korrelationskoeffizienten und Irrtumswahrscheinlichkeit (p); ns: nicht statistisch signifikant.

\begin{tabular}{|c|rr|r|r|}
\hline & \multicolumn{3}{|c|}{ Nächte mit Aufwachepisoden pro Woche } \\
\hline & 4 Monate & 8 Monate & 12 Monate \\
\hline $\begin{array}{c}\text { Fragebogenskala } \\
\text { „Hoffnungslosig- }\end{array}$ & $\mathrm{r}=0,085$ & $\mathrm{r}=-0,041$ & $\mathrm{n}=-0,006$ \\
keit““ & $\mathrm{ns}$ & $\mathrm{ns}$ & .956 \\
& $\mathrm{n}=92$ & $\mathrm{n}=87$ & $\mathrm{n}=83$ \\
\hline
\end{tabular}

Anmerkung: Mitgeteilt sind punktbiseriale Korrelationskoeffizienten und Irrtumswahrscheinlichkeit (p); ns: nicht statistisch signifikant.

\begin{tabular}{|c|r|r|r|}
\hline & \multicolumn{3}{|l|}{ Aufwachepisoden pro Nacht } \\
\hline & 4 Monate & 8 Monate & 12 Monate \\
\hline Fragebogenskala & $\mathrm{r}=0,132$ & $\mathrm{r}=0,095$ & $\mathrm{r}=0,067$ \\
"Trait- Angst“" & $\mathrm{ns}$ & $\mathrm{ns}$ & $\mathrm{ns}$ \\
& $\mathrm{n}=92$ & $\mathrm{n}=89$ & $\mathrm{n}=86$ \\
\hline Fragebogenskala & $\mathbf{r}=\mathbf{0 , 2 0 0}$ & $\mathbf{r}=\mathbf{0 , 1 9 4}$ & $\mathrm{r}=0,125$ \\
„Überfürsorge aus & $\mathbf{p}<\mathbf{0 , 0 5 7}$ & $\mathbf{p}<\mathbf{0 , 0 7 6}$ & $\mathrm{ns}$ \\
Angst“" & $\mathbf{n = 9 1}$ & $\mathbf{n}=\mathbf{8 5}$ & $\mathrm{n}=85$ \\
\hline
\end{tabular}

Anmerkung: Mitgeteilt sind punktbiseriale Korrelationskoeffizienten und Irrtumswahrscheinlichkeit (p); ns: nicht statistisch signifikant. 


\begin{tabular}{|c|r|rr|r|}
\hline & \multicolumn{3}{|l|}{ Nächte mit Aufwachepisoden pro Woche } \\
\hline & 4 Monate & 8 Monate & 12 Monate \\
\hline Fragebogenskala & $\mathrm{r}=0,029$ & $\mathrm{r}=-0,654$ & $\mathrm{r}=-0,103$ \\
„Trait- Angst“" & $\mathrm{ns}$ & $\mathrm{ns}$ & $\mathrm{ns}$ \\
\hline $\begin{array}{c}\text { Fragebogenskala } \\
\text { „Überfürsorge aus }\end{array}$ & $\mathrm{n}=0,061$ & $\mathrm{n}=88$ & $\mathrm{n}=85$ \\
Angst“" & $\mathrm{ns}$ & $\mathrm{r}=-0,138$ & $\mathrm{r}=-0,082$ \\
$\mathrm{n}=90$ & $\mathrm{~ns}$ & $\mathrm{~ns}$ \\
\hline
\end{tabular}

Anmerkung: Mitgeteilt sind punktbiseriale Korrelationskoeffizienten und Irrtumswahrscheinlichkeit (p); ns: nicht statistisch signifikant.

\begin{tabular}{|c|r|r|r|}
\hline & \multicolumn{3}{|l|}{ Aufwachepisoden pro Nacht } \\
\hline & 4 Monate & 8 Monate & 12 Monate \\
\hline über allen De- & $\mathbf{r}=\mathbf{0 , 2 3 4}$ & $\mathrm{r}=0,110$ & $\mathrm{r}=0,124$ \\
pressivitäts- und & $\mathbf{p}<\mathbf{0 , 0 2 5}$ & $\mathrm{ns}$ & $\mathrm{ns}$ \\
Angstskalen & $\mathbf{n = 9 2}$ & $\mathrm{n}=89$ & $\mathrm{n}=90$ \\
\hline
\end{tabular}

Anmerkung: Mitgeteilt sind punktbiseriale Korrelationskoeffizienten und Irrtumswahrscheinlichkeit (p); ns: nicht statistisch signifikant

\begin{tabular}{|c|r|r|r|}
\hline & \multicolumn{3}{|c|}{ Nächte mit Aufwachepisoden pro Woche } \\
\hline & 4 Monate & 8 Monate & 12 Monate \\
\hline$\sum$ über allen De- & $\mathrm{r}=0,090$ & $\mathrm{r}=-0,033$ & $\mathrm{r}=-0,029$ \\
pressivitäts- und & $\mathrm{ns}$ & $\mathrm{ns}$ & $\mathrm{ns}$ \\
Angstskalen & $\mathrm{n}=91$ & $\mathrm{n}=88$ & $\mathrm{n}=89$ \\
\hline
\end{tabular}

Anmerkung: Mitgeteilt sind punktbiseriale Korrelationskoeffizienten und Irrtumswahrscheinlichkeit (p); ns: nicht statistisch signifikant 
Tabelle 18: Häufigkeitstabelle hinsichtlich des Geschlechts der Säuglinge

\begin{tabular}{|l|r|r|}
\hline & Häufigkeit & Prozent \\
\hline männlich & 58 & 57,4 \\
weiblich & 43 & 42,6 \\
Gültig Ge- & 101 & 100 \\
samt & & \\
Fehlend & 0 & \\
GESAMT & 101 & \\
\hline
\end{tabular}

Anmerkung: 3 Fälle konnten bei den längsschnittlichen statistischen Analysen nicht berücksichtigt werden (vgl. Kapitel 4.1.4.)

Tabelle 19: Häufigkeitstabelle zum Alter der Mutter

\begin{tabular}{|c|c|c|}
\hline & Häufigkeit & Prozent \\
\hline 21 & 1 & 1 \\
\hline 22 & 6 & 6,1 \\
\hline 23 & 2 & 2 \\
\hline 24 & 4 & 4,1 \\
\hline 25 & 4 & 4,1 \\
\hline 26 & 8 & 8,2 \\
\hline 27 & 11 & 11,2 \\
\hline 28 & 8 & 8,2 \\
\hline 29 & 9 & 9,2 \\
\hline 30 & 16 & 16,3 \\
\hline 31 & 10 & 10,2 \\
\hline 32 & 7 & 7,1 \\
\hline 33 & 2 & 2 \\
\hline 34 & 2 & 2 \\
\hline 35 & 3 & 3,1 \\
\hline 36 & 3 & 3,1 \\
\hline 37 & 1 & 1 \\
\hline 40 & 1 & 1 \\
\hline Gültig Gesamt & 98 & 100 \\
\hline Fehlend & 0 & \\
\hline GESAMT & 98 & \\
\hline Mittelwert & 28,81 & \\
\hline Median & 29,00 & \\
\hline Standardabweichung & 3,74 & \\
\hline Minimum & 21 & \\
\hline Maximum & 40 & \\
\hline
\end{tabular}


Tabelle 20: Häufigkeitstabelle zum Alter des Vaters

\begin{tabular}{|c|c|c|}
\hline & Häufigkeit & Prozent \\
\hline 20 & 1 & 1 \\
\hline 21 & 2 & 2 \\
\hline 22 & 2 & 2 \\
\hline 25 & 3 & 3,1 \\
\hline 26 & 5 & 5,1 \\
\hline 27 & 1 & 1 \\
\hline 28 & 3 & 3,1 \\
\hline 29 & 17 & 17,3 \\
\hline 30 & 6 & 6,1 \\
\hline 31 & 10 & 10,2 \\
\hline 32 & 12 & 12,2 \\
\hline 33 & 9 & 9,2 \\
\hline 34 & 9 & 9,2 \\
\hline 35 & 2 & 2 \\
\hline 36 & 3 & 3,1 \\
\hline 37 & 4 & 4,1 \\
\hline 38 & 2 & 2 \\
\hline 39 & 2 & 2 \\
\hline 41 & 1 & 1 \\
\hline 42 & 1 & 1 \\
\hline 43 & 1 & 1 \\
\hline 46 & 1 & 1 \\
\hline 49 & 1 & 1 \\
\hline Gültig Gesamt & 98 & 100 \\
\hline Fehlend & 0 & \\
\hline GESAMT & 98 & \\
\hline Mittelwert & 31,56 & \\
\hline Median & 31,00 & \\
\hline Standardabweichung & 4,89 & \\
\hline Minimum & 20 & \\
\hline Maximum & 49 & \\
\hline
\end{tabular}


Tabelle 21: Häufigkeitstabelle zum Bildungsniveau der Mutter

\begin{tabular}{|l|r|r|}
\hline & Häufigkeit & Prozent \\
\hline Hauptschule & 7 & 7,1 \\
Realschule & 32 & 32,7 \\
Abitur & 40 & 40,8 \\
Hoch- oder Fach- & 19 & 19,4 \\
hochschulabschluss & & \\
Gültig Gesamt & 98 & 100 \\
Fehlend & 0 & \\
GESAMT & 98 & \\
& & \\
Mittelwert & 2,72 & \\
Median & 3,00 & \\
Standardabweichung & 0,86 & \\
& &
\end{tabular}

Tabelle 22: Häufigkeitstabelle zum Bildungsniveau des Vaters

\begin{tabular}{|l|r|r|}
\hline & Häufigkeit & Prozent \\
\hline Hauptschule & 8 & 8,2 \\
Realschule & 29 & 29,6 \\
Abitur & 41 & 41,8 \\
Hoch- oder Fach- & 20 & 20,4 \\
hochschulabschluss & & \\
Gültig Gesamt & 98 & 100 \\
Fehlend & 0 & \\
GESAMT & 98 & \\
& & \\
Mittelwert & 2,74 & \\
Median & 3,00 & \\
Standardabweichung & 0,88 & \\
\cline { 1 - 1 } & &
\end{tabular}




\section{Anhang B: Literaturverzeichnis}

Anders, T.F., Goodlin-Jones, B. \& Sadeh, A. (2000). Sleep disorders. In. C.H. Zeanah (Ed.), Handbook of infant mental health (2nd ed., pp. 326-338). New York: Guilford Press.

Bayley, N. (1993). Bayley Scales of Infant Development. Second Edition. San Antonio: The Psychological Corporation.

Bridges, L.J., Grolnick, W.S. \& Connell, J.P. (1997). Infant emotion regulation with mothers and fathers. Infant Behavior and Development, 20, 47-57.

Burnham, M.M., Goodlin-Jones, B.L.,Gaylor, E.E., Anders, T.F. (2002). Nighttime sleep-wake patterns and self-soothing from birth to one year of age: a longitudinal intervention study. J Child Psychol Psychiatry, 43(6), 713-25.

Call, J. (1983). Toward a nosology of psychiatric disorders in infancy. In Call, J.; Galenson, E.; Tyson, R. L. (Eds.), Frontiers of infant psychiatry. Basic Books, New York.

Campbell, S.B., Ewing, L.J., Breaux, A.M. \& Szumowski, E.K. (1986). Parent-referred problem three-year-olds: Follow-up at school entry. Journal of Child Psychology and Psychiatry, 27, 473-488.

Carey, W.B. \& McDevitt, S.C. (1978). Revision of the infant temperament questionnaire. Paediatrics, 61, 735-739.

Cicchetti, D. \& Toth, S.L. (1997). Transactional ecological systems in developmental psychopathology. In S.S. Luthar, J.A. Burack, D. Cicchetti \& J.R. Weisz (Eds.), Developmental psychopathology. Perspectives on adjustment, risk and disorder (pp. 273- 313). Cambridge: Cambridge University Press. 
Cicchetti, D., Rogosch, F.A. \& Toth, S.L. (1997). Ontogenesis, depressotypic organization and the depressive spectrum. In S.S. Luthar, J.A. Burack, D. Cicchetti \& J.R. Weisz (Eds.), Developmental psychopathology. Perspectives on adjustment, risk and disorder (pp. 273- 313). Cambridge: Cambridge University Press.

Cowen, E.L, Work, W.C. \& Wyman, P.A. (1997). The Rochester Child Resilience Project (RCRP): Facts found, lessons learned, future directions divined. In S.S. Luthar, J.A. Burack, D. Cicchetti \& J.R. Weisz (Eds.), Developmental psychopathology. Perspectives on adjustment, risk, and disorder (527- 547). Cambridge: Cambridge University Press.

DeGangi, G.A., DiPietro, J.A., Greenspan, S.I. \& Porges, S. (1991). Psychophysiological characteristics of the regulatory disordered infant. Infant Behavior Development, 14, 37- 50.

Deutsche Gesellschaft für Kinder- und Jugendpsychiatrie und Psychotherapie u.a. (Hrsg.): Leitlinien zur Diagnostik und Therapie von psychischen Störungen im Säuglings-, Kindes- und Jugendalter. Deutscher Ärzte Verlag, 2. überarbeitete Auflage 2003 - ISBN: 3-7691-0421-8.

Dilling, H., Mombour, W. und Schmidt, M. H. (Hrsg.). (1991). Internationale Klassifikation psychischer Störungen. ICD-10 Kapitel V (F). Bern: Verlag Hans Huber.

Egeland, B.R., Carlson, E. \& Sroufe, L.A. (1993). Resilience as process. Development and Psychopathology, 5, 517-528.

Emde, R.N. (1985). Assessment of infancy disorders. In M. Rutter, L. Hersov (Eds.), Child and adolescent psychiatry (2d ed.). Blackwell, Oxford.

Emde, R. N. (1987). Infant mental health: Clinical dilemmas, the expansion of meaning and opportunities. In J.D. Osofsky (Eds.), Handbook of infant development (2d ed.) 1297- 1320. Wiley, New York. 
Emde, R. N. and Score, J.F. (1984). Infancy: Perspectives on Normality. In D. Offer, M. Sabshin (Eds.), Normality and the life cycle (pp. 3-29). Basic Books, New York.

Engfer, A. (1984). Entwicklung punitiver Mutter-Kind Interaktionen im soziökologischen Kontext Arbeitsbericht an die DFG; Inst. Für Psychologie, Uni.München.

Esser, G., Dinter, R., Jörg, M., Rose, F., Villalba, P., Laucht, M. \& Schmidt, M.H. (1993). Bedeutung und Determinanten der frühen Mutter-Kind-Beziehung. Zeitschrift für Psychosomatische Medizin und Psychoanalyse, 39, 246-264.

Esser, G., Scheven, A., Petrova, A., Laucht, M. \& Schmidt, M.H. (1989). Mannheimer Beurteilungsskala zur Erfassung der Mutter-Kind-Interaktion im Säuglingsalter (MBS-MKI-S). Zeitschrift für Kinder und Jugendpsychiatrie, 17, 185-193.

Fegert, J., Schulz, J., Bermann, R., Tacke, U., Bergmann, K. \& Wahn, U. (1997). Schlafverhalten in den ersten drei Lebensjahren. Praxis der Kinderpsychologie und Kinderpsychiatrie, 17, 185-93.

Ficca, G., Fagioli, I, Giganti, F. \& Salzarulo, P. (1999). Spontaneous awakenings from sleep in the first year of life. Early Hum Dev,. 55(3), 219-28.

Field, T. (1994). The effects of mothers's physical and emotional unavailability on emotion regulation. In N.A. Fox (Ed.), The development of emotions regulation: Biological and behavioral considerations. Monographs of the society for Research in Child Development, 59 (2-3, Serial No. 240), 208-227.

Fish, M., \& Stifter, C. (1999). Parenting Attitudes and behaviors in rural Appalachian mothers: A comparison study. Journal of Rural Community Psychology, Vol E-2(1) [on-line]. Available: http://www.marshall.edu/jrcp.

Gaylor, E.E., Goodlin-Jones, B.L., Burnham, M.M. \& Anders, T.F. (1998). Maternal perception of night awakenings and infant self-soothing behavior during the first year of life. Paper presented at the International Conference of Infant Studies, Atlanta, USA, 2.-5. April 1998. (Kap. 14). 
Gaylor, E.E., Goodlin-Jones, B.L. \& Anders, T.F. (2001). Classification of young children's sleep problems: a pilot study. J Am Acad Child Adolesc Psychiatry, 40(1), $61-7$.

Hiscock, H. \& Wake, M. (2001). Infant sleep problems and postnatal depression: a community-based study. Pediatrics, 107(6), 1317-22.

Hiscock, H. \& Wake, M. (2002). Randomised controlled trial of behavioural infant sleep intervention to improve infant sleep and maternal mood. BMJ, 324(7345), 1062-5.

Hoppenbrouwers, T., Hodgman, J., Arakawa, K., Geidel, S.A. \& Sterman, M.B.(1988). Sleep and waking states in infancy: normative studies. Sleep, 11(4), $387-401$.

Huizink, A.C., Mulder, E.J. \& Buitelaar, J.K. (2004). Prenatal stress and risk for psychopathology: specific effects or induction of general susceptibility? Psychol Bull, $130(1), 115-42$.

Jensen, P.S., Bloedau, L., Degroot, J., Ussery, T. \& Davis, H. (1990). Children at risk: Risk factors and child sympthomatology. Journal of the American Academy of Child and Adolescent Psychiatry, 29, 51- 59.

Kazdin, A.E., Kraemer, H.C., Kessler, R.C., Kupfer, D.J. \& Offord, D.R. (1997). Contribution of risk-factor research to developmental psychopathology. Clinical Psychology Review, 17, 375- 406.

Kirjavainen, J., Kirjavainen,, T., Huhtala, V., Lehtonen, L., Korvenranta, H. \& Kero, P. (2001). Infants with colic have a normal sleep structure at 2 and 7 months of age. $J$ Pediatr, 138(2), 218-23. 
Kogan, N. \& Carter, A.S. (1996). Mother- infant reengagement following the still-face: The role of maternal emotional availability in infant affect regulation. Infant Behavior and Development, 19, 359- 370.

Kopp, C. (1989). Regulation of distress and negative emotions: A developmental view. Developmental Psychology, 25, 343-354.

Krampen, G. (1979). Hoffnungslosigkeit bei stationären Patienten- Ihre Messung durch einen Kurzfragebogen (H-Skala). Medizinische Psychologie, 5, 39-49.

Laucht, M., Esser, G. \& Schmidt, M.H. (1993). Adverse temperamental characteristics and early behaviour problems in 3-month-old infants born with different psychosocial and biological risks. Acta Pädopsychiatrica, 56, 19-24.

Laucht, M., Esser, G. \& Schmidt, M.H. (1997). Wovor schützen Schutzfaktoren? Anmerkungen zu einem populären Konzept der modernen Gesundheitsforschung. Zeitschrift für Entwicklungspsychologie und Pädagogische Psychologie, 29, 260270.

Laucht, M., Esser, G. \& Schmidt, M.H. (1998). Risiko- und Schutzfaktoren der frühkindlichen Entwicklung: Empirische Befunde. Zeitschrift für Kinder- und Jugendpsychiatrie, 26, 6- 20.

Laucht, M., Esser, G., Schmidt M.H. (1992). Verhaltensauffälligkeiten bei Säuglingen und Kleinkindern. Zeitschrift für Kinder- und Jungendpsychiatrie, 20, 22-33.

Laucht, M., Esser, G., Schmidt, M.H., Ihle, W., Marcus, A., Stöhr, R.-M. \& Weindrich, D. (1996). Viereinhalb Jahre danach: Mannheimer Risikokinder im Vorschulalter. Zeitschrift für Kinder- und Jugendpsychiatrie, 24, 67- 81.

Laux, L., Glanzmann, P., Schaffer, P. \& Spielberger, C.D. (1981). Das State-TraitAngstinventar Weinheim: Beltz. 
Louis, J., Cannard, C., Bastuji, H. \& Challamel, M.J. (1997). Sleep ontogenesis revisited: a longitudinal 24-hour home polygraphic study on 15 normal infants during the first two years of life. Sleep, 20(5), 323-33.

Lozoff, B., Wolf, A.W. \& Davis, N.S. (1985). Sleep problems seen in pediatric practice. Pediatrics, 75, 477- 483.

Matheny, A.P., Dolan, A.B. \& Wilson, R.S. (1974). Bayley's infant behavior record: Relations between behaviors and mental test scores. Developmental Psychology, 10, 696-702.

Mayseless, O. (1998). Maternal caregiving strategy: A distinction between the ambivalent and the disorganized profile. Infant Mental Health Journal, 19, 20-33.

Mc Loyd, V.C. \& Wilson, L. (1991). The strain of living poor: Parenting, social support, and child mental health. In A.C. Huston (Ed.), Children in poverty: Child development and public policy (pp105- 135). Cambridge: Cambridge University Press.

Minde, K., Popiel, K., Leos, N., Falkner, S., Parker, K. \& Handley-Derry, M. (1993). The evaluation and treatment of sleep disturbances in young children. Journal of child Psychology and Psychiatry, 34, 521-533 (Kap.14).

Moffitt, T.E. (1993). The neuropsychology of conduct disorder. Development and Psychopathology, 5, 135-151.

Moffitt, T.E., Caspi, A., Dickson, N., Silva, P. \& Stanton, W. (1996). Childhood-onset versus adolescent-onsent antisocial conduct problems in males: natural history form age 3 to 18 years. Development Psychopathology, 8, 399-424.

Murray, L. \& Cooper, P.J. (Eds.). (1997). Postpartum depression and child development. New York: Guilford Press. 
Newcomb, M.D. \& Felix-Ortiz, M. (1992). Multiple protective and risk factors for drug use and abuse: Cross-sectional and prospective findings. Journal of Personality and Social Psychology, 63, 280-296.

Papousek, M. (1994). Vom ersten Schrei zum ersten Wort. Bern: Huber.

Pauli-Pott, U., Mertesacker, B. \& Beckmann, D. (2003). Ein Fragebogen zur Erfassung des "frühkindlichen Temperaments" im Elternurteil [A questionnaire for assessment of "temprament in early childhood" as judged by parents]. Z Kinder Jugendpsychiatr Psychother, 31(2), 99-110.

Pauli-Pott, U., Mertesacker, B. \& Beckmann, D. (2005) Ein Vergleich unterschiedlicher Erfassungsmethoden der frühkindlichen Emotionalität. Z Kinder Jugendpsychiatr Psychother, 33 (2).

Pauli-Pott, U., Mertesacker, B. \& Beckmann, D. (2004). Predicting the development of infant emotionality from maternal characteristics. Dev Psychopathol, 16(1) 1942.

Pauli-Pott, U. (2001). Frühkindliche Temperamentsmerkmale- normative Stabilität und Bedingungen ihres Wandels. Habilitationsschrift. Fachbereich Humanmedizin, Giessen.

Petermann, F. \& Scheithauer, H. (1998). Aggressives und antisoziales Verhalten im Kindes- und Jugendalter. In F. Petermann, M. Kusch \& K. Niebank, Entwicklungspsychopathologie. Ein Lehrbuch (S. 243- 295). Weinheim: Psychologie Verlags Union.

Pollock, J.I. (1992). Predictors and long-term associations of reported sleeping difficulties in infancy. J Reprod Infant Psychol, 10, 151-68.

Rennen-Allhoff, B. (1990). Testgüte von Entwicklungstests- Ergebnisse der Marburger Säuglingsstudie Köln: Dt. Ärzte-Verlag. 
Richman, N., Stevenson J. \& Graham P.J. (1982). Pre-school to school. A behavioral study. New York: Academic Press.

Roth, K., Eisenberg, N. \& Sell, E.R. (1984). The relation of preterm and full-term infants temperament to test-taking behaviors and developmental status. Infant Behavior and Development, 7, 495-505.

Rothbart, M.K. \& Bates, J.E. (1998). Temperament. In N. Eisenberg (Vol. Ed.), \& W. Damon (Series Ed.), Handbook of Child Psychology: Vol. 4. Social, emotional and personality development (5 th ed., pp. 105-176). New York: Wiley.

Rothbart, M.K. (1981). Measurement of temperament in infancy. Child Development, $52,569-578$.

Ruff, H.A. \& Rothbart, M.K. (1996). Attention in early development: Themes and variations. New York: Oxford University Press.

Rutter, M. (1988). Studies of psychosocial risk: The power of longitudinal data. Cambridge University Press, Cambridge.

Rutter, M. (1985). Resilience in the face of adversity. Protective factors and resistance to psychiatric disorder. British Journal of Psychiatry, 147, 598- 611.

Rutter, M. (1990). Psychosocial resilience and protective mechanisms. In J. Rolf, A.S. Masten, D. Cicchetti, K.H. Nuechterlein \& S. Weintraub (Eds.), Risk and protective factors in the development of psychopathology (pp. 181 -214). New York: Cambridge University Press.

Sadeh, A. (1996). Evaluating night wakings in sleep-disturbed infants: a methodological study of parental reports and actigraphy. Sleep, 19(10) 757-62.

Sadeh, A., Dark, I. \& Vohr, B.R. (1996). Newborns' sleep-wake patterns: the role of maternal, delivery and infant factors. Early Hum Dev, 44(2),113-26. 
Sarimski, K. \& Papousek, M. (2000). Eltern-Kind-Beziehung und die Entwicklung von Regulationsstörungen. In Petermann, F., Niebank, K. \& Scheithauer, H. (Hrsg.), Risiken der frühkindlichen Entwicklung. Entwicklungspsychopathologie der ersten Lebensjahre (S. 199- 222). Göttingen: Hogrefe.

Saß, H., Wittchen, H.-U. und Zaudig, M. (Hrsg.).(1996). Diagnostisches und statistisches Manual psychischer Störungen (DSM IV). Göttingen: Hogrefe.

Scheithauer, H. \& Petermann, F. (1999). Aggression. In F. Petermann (Hrsg.) Lehrbuch der Klinischen Kinderpsychologie und -psychotherapie (S. 192-230); 4. Völlig veränd. Auflage). Göttingen: Hogrefe.

Scheithauer, H. \& Petermann, F. (2000). Aggression. In F. Petermann (Hrsg.), Lehrbuch der Klinischen Kinderpsychologie und -psychotherapie (S.192-230; 4. Völlig veränd. Auflage). Göttingen: Hogrefe.

Scheithauer, H., Niebank, K. \& Petermann, F. (2000). Biopsychosoziale Risiken in der frühkindlichen Entwicklung: Das Risiko- und Schutzfaktorenkonzept aus entwicklungspsychopathologischer Sicht. In Petermann, F., Niebank, K. \& Scheithauer, H. (Hrsg.), Risiken der frühkindlichen Entwicklung. Entwicklungspsychopathologie der ersten Lebensjahre (S. 65-97). Göttingen: Hogrefe

Scher, A. (2001). Attachment and sleep: a study of night waking in 12-month-old infants. Dev Psychobiol, 38(4), 274-85.

Scher, A., Tirosh, E. \& Lavie, P. (1998). The relationship between sleep and temperament revisited: evidence for 12-month-olds: a research note. J Child Psychol Psychiatry, 39(5), 785-8.

Skuse, D., Pickles, A., Wolke, D. \& Reilly, S. (1992). Postnatal growth and mental development: evidence for a „sensitive period”. J Child Psychol Psychiatry, 35, $521-45$. 
Skuse, D., Reilly, S. \& Wolke, D. (1994). Psychological adversity and growth during infancy. European Journal of Clinical Nutrition, 48, 113-130 (Kap.14).

Spieker, S. J., \& Booth, C. L. (1988). Maternal antecedents of attachment quality. In J. Belsky \& T. Nezworski (Eds.), Clinical implications of attachment. Hillsdale, NJ: Erlbaum.

Sroufe, L.A. (1996). Emotional development: The organization of emotional life in the early years. New York: Cambridge University Press.

Stattin, H. \& Magnusson, D. (1996). Antisocial behavior: A holistic approach. Development and Psychopathology, 8, 617-645.

St. James-Roberts, I.S., Conroy S. \& Hurry, J. (1997) Links between infant crying and sleep-waking at six weeks of age. Early Hum Dev, 48(1-2), 143-52.

Thompson, R.A. (1994). Emotion regulation: A theme in search of definition. Monographs of the Society for Research in Child Development, 59(2-3, Serial No. 240).

Thunström, M. (1999). Severe sleep problems among infants in a normal population in Sweden: prevalence, severity and correlates. Acta Paediatr, 88(12), 1356-63.

Thunström, M. (2002). Severe sleep problems in infancy associated with subsequent development of attention-deficit/hyperactivity disorder at 5.5 years of age. Acta $\mathrm{Pa}-$ ediatr, 91(5), 584-92.

Tronick, E.Z. (1989). Emotions and emotional communication in infants. American Psychologist, 44, 112-119.

Von Hofacker, N. \& Papousek, M. (1998). Disorder of excessive crying, feeding, and sleeping: The Munich Interdisciplinary Research and Intervention Program. Infant Mental Health Journal, 19 (2), 180-201 (Kap.5,12). 
von Hofacker, N., Jacubeit, T., Malinowski, M. \& Papousek, M. (1996). Diagnostik von Beeinträchtigungen der Mutter- Kind- Beziehung bei frühkindlichen Störungen der Verhaltensregulation. Kindheit und Entwicklung, 5, 160- 167. (Kap.5).

Walker, D., Greenwood, C., Hart, B., \& Carta, J. (1994). Prediction of school outcomes based on early language production and socioeconomic factors. Child Development, 65, 606-621.

Werner, E. E. \& Smith, R. S. (1992). Overcoming the Odds: High risk children from birth to adulthood. Ithaca, NY: Cornell University Press.

Wolke, D. (1994). Feeding and sleeping across the lifespan. In M. Rutter \& D. Hay (Eds.), Development through life: A handbook for clinicians (pp. 517- 557). Oxford: Blackwell scientific Publications (Kap. 14).

Wolke, D. (1998). Premature babies and the Special Care Baby Unit (SCBU)/ Neonatal Intensive Care Unit (NI-CU): Environmental, medical and developmental considerations. In C.A. Niven \& A. Walker (Eds.), Current issues in infancy and parenthood (Vol. 3,pp. 255-281). Oxford: Butterworth- Heinemann (Kap. 14,22).

Wolke, D. (1999). Interventionen bei Regulationsstörungen. In: R. Oerter, C. von Hagen, G. Röper (Eds.), Klinische Entwicklungspsychologie (S. 351- 380). Weinheim, Germany: Beltz PVU.

Wolke, D., Meyer, R., Ohrt, B. \& Riegel, K. (1995). The incidence of sleeping problems in preterm and fullterm infants discharged from special units: An epidemiological longitudinal study. Journal of Child Psychology and Psychiatry, 36, 203-223.

Wolke, D., Meyer, R., Ohrt, B. \& Riegel, K. (1995): Co-morbidity of crying and feeding problems with sleeping problems in infancy: concurrent and predictive associations. Early Development Parenting; 4, 191- 207.

Wolke, D., Ratschinski, G., Ohrt, B. \& Riegel, K. (1994). The cognitive outcome of very preterm infants may be poorer than often reported: An empirical investigation 
of how methodological issues make a big difference. European Journal of Pediatrics, 153, 906-915 (Kap. 22).

Wolke, D., Rizzo, P. \& Woods, S. (2002). Persistent Infant Crying and Hyperactivity Problems in Middle Childhood. Pediatrics, 109, 1054- 1060.

Wolke, D., Sohne, B., Riegel, K., Ohrt, B. \& Osterlund, K. (1998). An epidemiologic longitudinal study of sleeping problems and feeding experience of preterm and term children in southern Finland: comparison with a southern German population sample. Journal of Pediatrics, 133 (2), 224-31.

Zuckerman, B., Stevenson, J. \& Bailey, V. (1987). Sleep problems in early childhood: continuities, predictive factors, and behavioral correlates. Pediatrics, 80(5), 664-71. 


\section{Anhang C: Fragebögen}

Soziale Anamnese im Rahmen der Hausbesuche

Kurzinterview mit der Bezugsperson

Deutsch Adaptation des IBQ

Fragebögen zur Erhebung der mütterlichen Merkmale:

- Fragebogenskala „Depressivität“ und „Überfürsorge aus Angst“ aus dem EMKK von Engfer

- Fragebogenskala „Trait-Angst“ aus dem STAI von Laux et al.

- Fragebogenskala „Hoffnungslosigkeit“ von Krampen 


\section{$\underline{\text { Soziale Anamnese im Rahmen der Hausbesuche (T1) }}$}

Mutter

$\underline{\text { Alter: }}$

Schulbildung:

kein Abschluß

Hauptschule

Handelsschule

Realschule

Abitur

Fach-/Hochsch.
Vater

$\underline{\text { Alter: }}$

Schulbildung:

kein Abschluß

Hauptschule

Handelsschule

Realschule

Abitur

Fach-/Hochsch.

Abgeschlossene Berufsausbildung:

ja

nein ja

nein

Beruf:

Mutter berufstätig bis Mutterschutzzeit: ja

nein

Studium/Ausbildung

Mutter zur Zeit berufstätig:

nein

halb

voll

Studium/Ausbildung

Vater zur Zeit berufstätig:

nein

halb

voll

Studium/Ausbildung 
Größe der Wohnung $\left(\mathrm{m}^{2}\right)$ :

Anzahl der Zimmer:

Anzahl der Personen in der Wohnung (incl. Baby):

Schwangerschaft ungewollt: $\quad$ nein

Vergangenheit der Eltern:

ja (d.h. ernsthaft Abbruch erwogen)

Heimaufenthalte

aufgew. in 1-Eltern-Familie

Vater

Mutter keiner

körperliche Erkrankungen

Vater

Mutter keiner

psychische Erkrankungen

Vater

Mutter keiner

Vater Mutter keiner

\section{$\underline{\text { Aktuell: }}$}

Liegen Belastungen in der Familie vor? (z.B. Krankheit, Pflegefall, Arbeitslosigkeit, finanzielle Schwierigkeiten, Hausbau)

$$
\text { nein }
$$$$
\text { ja }
$$

Dauer der Partnerschaft: Monate Jahre

Versorgung des Babys:

Wieviel Stunden täglich beschäftigt sich der Vater mit dem Baby?

wochentags ca. wochenends ca. Std. Std.

Wie ist die Versorgung des Babys in Ihrer Partnerschaft aufgeteilt?

Vater: etwa gleich Partnerin macht mehr ich mache mehr Mutter: etwa gleich Partner macht mehr ich mache mehr 
Sind Sie zufrieden mit dieser Aufteilung?

Vater: eher zufrieden eher unzufrieden

Mutter: eher zufrieden eher unzufrieden

Wieviele Stunden täglich wird das Baby von Dritter Person betreut? Std. 


\section{T1: Kurzinterview mit der Bezugsperson (Gesundheit des Kindes)}

1. Zeitraum seit der letzten Mahlzeit des Babys: Minuten.

2. Geburtsgewicht: Gramm.

3. Apgarwerte: 5 Minuten ; 10 Minuten

4. Geschlecht des Kindes: 0 männlich

0 weiblich

5. Geburtsdatum:

6. Schwangerschaftskomplikationen:

7. Geburtskomplikationen:

8. Wurden Auffälligkeiten oder Erkrankungen bei den Vorsorgeuntersuchungen festgestellt?

Nein Ja wenn ja, welche:

Jawenn ja, welche:

\section{U.hefteintragung}

(a,b,c; Nummer)

U1:

O

O

U2:

$\mathbf{O}$

O

U3:

O

O

9. Bei der U3:

Körpergewicht: Gramm.

Körpergröße: cm 
10. Anzahl der Arztbesuche mit dem Baby außer Vorsorgeuntersuchungen:

_ mal

nein ja

11. Kamen bisher Erkältungskrankheiten vor (Husten, Schnupfen)

$\mathbf{O} \mathbf{O}$

12. Ist das Baby derzeit erkältet?

$\mathbf{O} \mathbf{O}$

13. Kam bisher Fieber vor (Temp. > 37.5)?

O O

14. Hat das Baby derzeit Fieber?

O $\mathbf{O}$

\section{Fragen zur Ernährung des Kindes:}

15. Wird das Baby zur Zeit:

\section{$O$ voll gestillt $\quad O$ gestillt und zugefüttert $O$ nicht gestillt}

16. Bis zu welchem Alter des Kindes wurde gestillt? O nie, Wochen

17. Wurde in der letzten Woche die Ernährung des Kindes umgestellt (abgestillt oder zugefüttert)?
$\mathbf{O}$ nein
O ja

18. Ist eine Umstellung der Ernährung in dieser Woche geplant?
O nein
O ja 
19. Still- bzw. Fütterfrequenz: ca. alle Stunden.

20. Das ist

O sehr regelmäßig $\quad$ O öfter mal unregelmäßig $O$ sehr unregelmäßig

so.

21. Hatte das Baby Koliken (Attacken unberuhigbaren Schreiens insbes. am Nachmittag und Abend)?
O nein
O etwas
O deutlich

22. Hat das Baby derzeit Koliken?
O nein
O etwas
O deutlich 


\section{T2: Kurzinterview mit der Bezugsperson (Gesundheit des Kindes)}

1. Zeitraum seit der letzten Mahlzeit des Babys: Minuten.

2. Wurden Auffälligkeiten oder Erkrankungen bei den Vorsorgeuntersuchungen festgestellt?

Nein Ja wenn ja, welche:

U.hefteintragung

(a,b,c; Nummer)

U4:

$\mathbf{O}$

$\mathbf{O}$

U5:

$\mathbf{O}$

$\mathbf{O}$

3. Bei der U5:

Körpergewicht: Gramm

Körpergröße: cm

4. Anzahl der Arztbesuche mit dem Baby außer Vorsorgeuntersuchungen im Intervall mal

nein ja

5. Kamen bisher Erkältungskrankheiten vor (Husten, Schnupfen)

O $\mathbf{O}$

6. Ist das Baby derzeit erkältet?

O $\mathbf{O}$

7. Kam seit letztem Termin Fieber vor (Temp. > 37.5)?

O O

8. Hat das Baby derzeit Fieber?

O $\mathbf{0}$ 


\section{Fragen zur Ernährung des Kindes:}

9. Wird das Baby zur Zeit:

\section{$O$ voll gestillt $\quad O$ gestillt und zugefüttert $O$ nicht gestillt}

10. Bis zu welchem Alter des Kindes wurde gestillt? O nie, Wochen

11. Wurde in der letzten Woche die Ernährung des Kindes umgestellt (abgestillt oder zugefüttert)?
O nein
O ja

12. Ist eine Umstellung der Ernährung in dieser Woche geplant?
O nein
O ja

13. Still- bzw. Fütterfrequenz: ca. alle Stunden.

14. Das ist

O sehr regelmäßig $\quad$ öfter mal unregelmäßig $O$ sehr unregelmäßig

so.

15. Wieviele Stunden schläft das Baby nachts (18-6 Uhr Schlafbeginn) ohne Unterbrechung?

\section{Stunden.}

16. Wie oft schläft das Baby am Tag: mal. 
17. Ist das

O sehr regelmäßig $\quad$ O öfter unregelmäßig $\quad$ O sehr unregelmäßig

so. 


\section{T3: Kurzinterview mit der Bezugsperson (Gesundheit des Kindes im Intervall)}

5. Zeitraum seit der letzten Mahlzeit des Babys: Minuten.

6. Wurden Auffälligkeiten oder Erkrankungen bei den Vorsorgeuntersuchungen festgestellt?

Nein Ja wenn ja, welche:$$
\text { Ja wenn ja, welche: }
$$

\section{U.hefteintragung}

(a,b,c; Nummer)

U6:

$\mathbf{O}$

$\mathbf{O}$

7. Bei der U6:

Körpergewicht:

Gramm

Körpergröße: cm

8. Anzahl der Arztbesuche mit Kind außer Vorsorgeunters. im Intervall mal

nein ja

5. Kamen seit letztem Termin Erkältungskrankheiten vor?

$\mathbf{O} \mathbf{O}$ (Husten, Schnupfen)

6. Ist das Kind derzeit erkältet?

O $\mathbf{O}$

7. Kam seit letztem Termin Fieber vor (Temp. > 37.5)?

O $\mathbf{O}$

8. Hat das Baby derzeit Fieber?

O O

9. Liegen im Moment andere Erkrankungen vor?

O

$\mathbf{O}$

wenn ja, welche 
wenn ja, welche

\section{Fragen zur Ernährung des Kindes:}

11. Wird das Baby zur Zeit:
O voll gestillt
$O$ gestillt und zugefüttert $O$ nicht gestillt

12. Bis zu welchem Alter des Kindes wurde gestillt? O nie, Wochen

13. Wurde in der letzten Woche die Ernährung des Kindes umgestellt (abgestillt oder zugefüttert)?
O nein
O ja

14. Ist eine Umstellung der Ernährung in dieser Woche geplant?
O nein
$\mathbf{O} \mathbf{j a}$

15. Still- bzw. Fütterfrequenz: ca. alle Stunden.

16. Das ist

O sehr regelmäßig $\quad$ öfter mal unregelmäßig $O$ sehr unregelmäßig so.

\section{Angaben zu den Schlafgewohnheiten}

17. Schläft Ihr Kind nachts durch? (23-6 Uhr)
O nein
O ja
wenn ja, seit wann (Lebensmonat)?

18. Wieviele Stunden schläft das Kind nachts (18-6 Uhr Schlafbeginn) ohne 
Unterbrechung?

\section{Stunden.}

19. In wie vielen Nächten pro Woche wacht Ihr Kind nachts auf?
ca.___Nächte

20. Wie oft wacht ihr Kind pro Nacht auf? mal.

21. Kommt es vor, dass es dann länger als $20 \mathrm{~min}$. wach ist?
O nein
$\mathbf{O} \mathbf{j a}$

22. Benötigt ihr Kind in der Regel mehr als 30 min. zum Einschlafen?
O nein
O ja

23. Kommt es vor, dass das Kind im Elternbett schläft? (auch wenn nachts dahin geholt)
O nein
O ja

16. Wie oft schläft das Baby am Tag: mal.

17. Ist das

O sehr regelmäßig $\quad$ o öfter unregelmäßig $\quad$ O sehr unregelmäßig

so. 
Deutsche Adaptation des IBQ

\title{
Fragebogen zum kindlichen Verhalten
}

Bitte lesen Sie die folgenden Hinweise sorgfältig:

Sie finden in diesem Fragebogen Beschreibungen kindlicher Verhaitensweisen. Geben Sie bitte für jede dieser Verhaltensweisen an, wie oft sich $\mathrm{Thr}$ Kind in der letzten Woche so verhalten hat.

Kreuzen Sie dazu bitte eine der Zahlen an, die immer neben der Beschreibung des Verhaltens stehen.

Diese Zahlen bedeuten. wie oft Sie das Verhalten in der letzten Woche beobachtet haben:

\author{
(1)...nie \\ (2)....sehr selten \\ (3)....weniger als bei der Hälfte der Gelegenheiten \\ (4)....ungefähr bei der Hälfte der Gelegenheiten \\ (5)....bei mehr als der Hälfte der Gelegenheiten \\ (6)....fast immer \\ (7)....immer
}

(x)....Die Situation kam nicht vor

(x): Die „kam nicht vor"-Spalte sollten Sie ankreuzen, wenn Sie das Baby während der letzten Woche nicht in der beschriebenen Situation beobachten konnten. Zum Beispiel, wenn die Situation, daß Thr Baby auf sein Essen warten mußte, in der letzten Woche nicht vorgekommen ist. "Kam nicht vor" ist etwas anderes als ,nie“".

"Nie" sollten Sie benutzen, wenn die Situation zwar vorkam, das Baby sich aber nie so wie beschrieben verhalten hat: Zum Beispiel, wenn das Baby auf sein Essen warten mußte, aber wälırend des Wartens niemals laut schrie.

Bitte achten Sie darauf, daß Sie bei jeder Frage eine Zahl ankreuzen!

Vielen Dank! 


\section{Füttern}

Wenn Ihr Baby auf sein Essen oder Getränk in der letzten Woche warten mußte, wie oft ...

\begin{tabular}{|l|c|c|c|c|c|c|c|c|}
\hline & $\begin{array}{l}\text { kam } \\
\text { nicht } \\
\text { vor }\end{array}$ & nie & $\begin{array}{l}\text { sehr } \\
\text { selten }\end{array}$ & $\begin{array}{l}\text { weniger } \\
\text { als } \\
\text { Hallte }\end{array}$ & $\begin{array}{l}\text { Halitte } \\
\text { der } \\
\text { Geleg. }\end{array}$ & $\begin{array}{l}\text { mehr } \\
\text { als } \\
\text { Halfe }\end{array}$ & $\begin{array}{l}\text { tast } \\
\text { immer }\end{array}$ & immer \\
\hline (1) schien es ihm wenig auszumachen? & $\mathbf{x}$ & $\mathbf{1}$ & $\mathbf{2}$ & $\mathbf{3}$ & $\mathbf{4}$ & $\mathbf{5}$ & $\mathbf{6}$ & $\mathbf{7}$ \\
\hline (2) zeigte es leichtes Unbehagen? & $\mathbf{x}$ & $\mathbf{1}$ & $\mathbf{2}$ & $\mathbf{3}$ & $\mathbf{4}$ & $\mathbf{5}$ & $\mathbf{6}$ & $\mathbf{7}$ \\
\hline (3) schrie es laut? & $\mathbf{x}$ & $\mathbf{1}$ & $\mathbf{2}$ & $\mathbf{3}$ & $\mathbf{4}$ & $\mathbf{5}$ & $\mathbf{6}$ & $\mathbf{7}$ \\
\hline
\end{tabular}

Beim Füttern, wie oft hat Ihr Baby ...

\begin{tabular}{|c|c|c|c|c|c|c|c|c|}
\hline & $\begin{array}{l}\text { kam } \\
\text { nicht } \\
\text { vor } \\
\end{array}$ & nie & $\begin{array}{l}\text { sehr } \\
\text { selten }\end{array}$ & $\begin{array}{l}\text { weniger } \\
\text { als } \\
\text { Hâlfte } \\
\end{array}$ & $\begin{array}{l}\text { Hualte } \\
\text { der } \\
\text { Geieg. }\end{array}$ & \begin{tabular}{|l|} 
mehrr \\
als \\
Hailte \\
\end{tabular} & $\begin{array}{l}\text { fast } \\
\text { innmer }\end{array}$ & immer \\
\hline (4) rulhig gelegen oder dagesessen? & $\mathbf{x}$ & 1 & 2 & 3 & 4 & 5 & 6 & 7 \\
\hline $\begin{array}{l}\text { (5) mit den Armen gewedelt, sich } \\
\text { gewunden oder gestrampelt? }\end{array}$ & $\mathbf{x}$ & 1 & 2 & 3 & 4 & 5 & 6 & 7 \\
\hline $\begin{array}{l}\text { (6) gequengelt oder geschrien, wemn es } \\
\text { genug hatte? }\end{array}$ & $x$ & 1 & 2 & 3 & 4 & 5 & 6 & 7 \\
\hline
\end{tabular}

Wenn Ihr Baby etwas Neuartiges zu essen oder zu trinken bekam, wie oft hat das Baby ...

\begin{tabular}{|c|c|c|c|c|c|c|c|c|}
\hline & \begin{tabular}{|l|}
$\begin{array}{l}\text { kamn } \\
\text { nicht } \\
\text { vor }\end{array}$ \\
\end{tabular} & nie & $\begin{array}{l}\text { sehr } \\
\text { selten }\end{array}$ & $\begin{array}{l}\text { weniger } \\
\text { ais } \\
\text { Hallte } \\
\end{array}$ & \begin{tabular}{|l} 
Halifte \\
der \\
Geleg.
\end{tabular} & $\begin{array}{l}\text { mehr } \\
\text { als } \\
\text { Hälfle } \\
\end{array}$ & $\begin{array}{l}\text { fast } \\
\text { inmer }\end{array}$ & immer \\
\hline (7) es sofort akzeptiert? & $\mathbf{x}$ & 1 & 2 & 3 & 4 & 5 & 6 & 7 \\
\hline (8) es überhaupt nicht akzeptiert? & $\mathbf{x}$ & 1 & 2 & 3 & 4 & 5 & 6 & 7 \\
\hline
\end{tabular}

\section{Schlafen}

Wie oft hat Ihr Kind in der letzten Woche ...

\begin{tabular}{|c|c|c|c|c|c|c|c|c|}
\hline & $\begin{array}{l}\text { kam } \\
\text { nicht } \\
\text { vor }\end{array}$ & nie & $\begin{array}{l}\text { sehr } \\
\text { selten }\end{array}$ & $\begin{array}{l}\text { wentiger } \\
\text { als } \\
\text { ykilfe }\end{array}$ & $\begin{array}{l}\text { Halfer } \\
\text { der } \\
\text { Geleg. }\end{array}$ & $\begin{array}{l}\text { mehr } \\
\text { als } \\
\text { Halne }\end{array}$ & $\begin{array}{l}\text { fast } \\
\text { immer }\end{array}$ & immer \\
\hline $\begin{array}{c}\text { (9) abends vor dem Einschlafen nicht } \\
\text { gequengelt oder geschrien? }\end{array}$ & $\mathbf{x}$ & $\mathbf{1}$ & $\mathbf{2}$ & $\mathbf{3}$ & $\mathbf{4}$ & $\mathbf{5}$ & $\mathbf{6}$ & $\mathbf{7}$ \\
\hline $\begin{array}{c}\text { (10) nicht gequengelt oder geschrien bevor } \\
\text { es am Tag einschlief? }\end{array}$ & $\mathbf{x}$ & $\mathbf{1}$ & $\mathbf{2}$ & $\mathbf{3}$ & $\mathbf{4}$ & $\mathbf{5}$ & $\mathbf{6}$ & $\mathbf{7}$ \\
\hline
\end{tabular}


Während des Schlafens, wie oft hat Ihr Baby ...

\begin{tabular}{|l|c|c|c|c|c|c|c|c|}
\hline & $\begin{array}{l}\text { kam } \\
\text { nicht } \\
\text { vor }\end{array}$ & nie & $\begin{array}{l}\text { sehr } \\
\text { selten }\end{array}$ & $\begin{array}{l}\text { weniger } \\
\text { als } \\
\text { Hallte }\end{array}$ & $\begin{array}{l}\text { Hallte } \\
\text { der } \\
\text { Geleg. }\end{array}$ & $\begin{array}{l}\text { mehr } \\
\text { als } \\
\text { Hallfe }\end{array}$ & $\begin{array}{l}\text { fast } \\
\text { imm }\end{array}$ & immer \\
\hline (11) sich im Bett umher bewegt? & $\mathbf{X}$ & $\mathbf{1}$ & $\mathbf{2}$ & $\mathbf{3}$ & $\mathbf{4}$ & $\mathbf{5}$ & $\mathbf{6}$ & $\mathbf{7}$ \\
\hline (12) an der gleichen Stelle ruhig gelegen? & $\mathbf{x}$ & $\mathbf{1}$ & $\mathbf{2}$ & $\mathbf{3}$ & $\mathbf{4}$ & $\mathbf{5}$ & $\mathbf{6}$ & $\mathbf{7}$ \\
\hline
\end{tabular}

Wenn es aufwachte, wie oft ...

\begin{tabular}{|c|c|c|c|c|c|c|c|c|}
\hline & \begin{tabular}{|l} 
kam \\
nicht \\
vor
\end{tabular} & nie & \begin{tabular}{|l} 
sehr \\
selten
\end{tabular} & $\begin{array}{l}\text { weniger } \\
\text { als } \\
\text { Hälfte }\end{array}$ & $\begin{array}{l}\text { Hallfe } \\
\text { der } \\
\text { Geleg. }\end{array}$ & $\begin{array}{l}\text { mehr } \\
\text { als } \\
\text { Halte }\end{array}$ & \begin{tabular}{|l|}
$\begin{array}{l}\text { fast } \\
\text { immer }\end{array}$ \\
\end{tabular} & immer \\
\hline (13) quengelte oder schrie Thr Baby sofort? & $\mathbf{x}$ & 1 & 2 & 3 & 4 & 5 & 6 & 7 \\
\hline (14) spielte es ruhig im Bett? & $\mathrm{x}$ & 1 & 2 & 3 & 4 & 5 & 6 & 7 \\
\hline $\begin{array}{l}\text { (15) weinte es, wenn innerhalb weniger } \\
\text { Minuten niemand kam? }\end{array}$ & $\mathbf{x}$ & 1 & 2 & 3 & 4 & 5 & 6 & 7 \\
\hline
\end{tabular}

\section{Baden und An- bzw. Ausziehen}

Wenn Ihr Baby in der letzten Woche an- oder ausgezogen wurde, wie oft hat es ...

\begin{tabular}{|l|c|c|c|c|c|c|c|c|}
\hline & $\begin{array}{l}\text { kam } \\
\text { nicht } \\
\text { vor }\end{array}$ & nie & $\begin{array}{l}\text { sehr } \\
\text { seiten }\end{array}$ & $\begin{array}{l}\text { weniger } \\
\text { als } \\
\text { Hallfte }\end{array}$ & $\begin{array}{l}\text { ider } \\
\text { der } \\
\text { Geleg. }\end{array}$ & $\begin{array}{l}\text { mehr } \\
\text { als } \\
\text { Hallte }\end{array}$ & $\begin{array}{l}\text { Gast } \\
\text { immer }\end{array}$ & immer \\
\hline $\begin{array}{c}\text { (16) mit den Armen gewedelt oder } \\
\text { gestrampelt? }\end{array}$ & $\mathbf{x}$ & $\mathbf{1}$ & $\mathbf{2}$ & $\mathbf{3}$ & $\mathbf{4}$ & $\mathbf{5}$ & $\mathbf{6}$ & $\mathbf{7}$ \\
\hline $\begin{array}{c}\text { (17) sich gewunden und versucht } \\
\text { wegzukommen? }\end{array}$ & $\mathbf{x}$ & $\mathbf{1}$ & $\mathbf{2}$ & $\mathbf{3}$ & $\mathbf{4}$ & $\mathbf{5}$ & $\mathbf{6}$ & $\mathbf{7}$ \\
\hline \begin{tabular}{l} 
(18) gelächelt oder gelacht? \\
\hline
\end{tabular} & $\mathbf{x}$ & $\mathbf{1}$ & $\mathbf{2}$ & $\mathbf{3}$ & $\mathbf{4}$ & $\mathbf{5}$ & $\mathbf{6}$ & $\mathbf{7}$ \\
\hline
\end{tabular}

Wenn Ihr Baby in das Badewasser kam, wie oft ...

\begin{tabular}{|c|c|c|c|c|c|c|c|c|}
\hline & $\begin{array}{l}\text { kams } \\
\text { nicht } \\
\text { vor }\end{array}$ & nie & $\begin{array}{l}\text { sehr } \\
\text { seiten }\end{array}$ & $\begin{array}{l}\text { weniger } \\
\text { als } \\
\text { Haifte }\end{array}$ & $\begin{array}{l}\text { Hélite } \\
\text { der } \\
\text { Geleg. }\end{array}$ & \begin{tabular}{|l} 
mehrs \\
als \\
Halthe
\end{tabular} & $\begin{array}{l}\text { fast } \\
\text { immer }\end{array}$ & irmer \\
\hline $\begin{array}{l}\text { (19) ist es erschrocken (nach Luft } \\
\text { schnappen, Arme hochwerfen, sich } \\
\text { versteifen etc.)? }\end{array}$ & $\mathbf{x}$ & 1 & 2 & 3 & 4 & 5 & 6 & 7 \\
\hline (20) hat es gelächelt oder gelacht? & $x$ & 1 & 2 & 3 & 4 & 5 & 6 & 7 \\
\hline $\begin{array}{l}\text { (21) machte es einen überraschten } \\
\text { Eindruck? }\end{array}$ & $\mathbf{x}$ & 1 & 2 & 3 & 4 & 5 & 6 & 7 \\
\hline (22) hat es geplanscht und gestrampelt? & $x$ & 1 & 2 & 3 & 4 & 5 & 6 & 7 \\
\hline (23) hat es sich gedreht und gewunden? & $\mathbf{x}$ & 1 & 2 & 3 & 4 & 5 & 6 & 7 \\
\hline (24) hat es viel geplappent? & $x$ & 1 & 2 & 3 & 4 & 5 & 6 & 7 \\
\hline
\end{tabular}


Wenn sein Gesicht gewaschen wurde, wie oft hat Ihr Baby ...

\begin{tabular}{|l|l|l|l|l|l|l|l|c|}
\hline & $\begin{array}{l}\text { kam } \\
\text { nicht } \\
\text { vor }\end{array}$ & nie & $\begin{array}{l}\text { sehr } \\
\text { selten }\end{array}$ & $\begin{array}{l}\text { werniger } \\
\text { als } \\
\text { Hälfee }\end{array}$ & $\begin{array}{l}\text { Hälfte } \\
\text { der } \\
\text { Gelen. }\end{array}$ & $\begin{array}{l}\text { mehr } \\
\text { als } \\
\text { Halffe }\end{array}$ & $\begin{array}{l}\text { hast } \\
\text { immer }\end{array}$ & inmer \\
\hline (25) gelächelt oder gelacht? & $\mathbf{x}$ & $\mathbf{1}$ & $\mathbf{2}$ & $\mathbf{3}$ & $\mathbf{4}$ & $\mathbf{5}$ & $\mathbf{6}$ & $\mathbf{7}$ \\
\hline (26) gejammert oder geschrien? & $\mathbf{x}$ & $\mathbf{1}$ & $\mathbf{2}$ & $\mathbf{3}$ & $\mathbf{4}$ & $\mathbf{5}$ & $\mathbf{6}$ & $\mathbf{7}$ \\
\hline
\end{tabular}

Beim Haare- oder Kopfwaschen, wie oft hat das Baby ...

\begin{tabular}{|c|c|c|c|c|c|c|c|c|}
\hline & $\begin{array}{l}\text { kam } \\
\text { nicht } \\
\text { vor } \\
\end{array}$ & nie & $\begin{array}{l}\text { sehr } \\
\text { seften }\end{array}$ & $\begin{array}{l}\text { Weniger } \\
\text { als } \\
\text { Hallfte } \\
\end{array}$ & $\begin{array}{l}\text { Hältle } \\
\text { der } \\
\text { Geleg. }\end{array}$ & \begin{tabular}{|l|} 
mehr \\
als \\
Halfte \\
\end{tabular} & 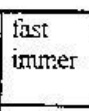 & immer \\
\hline (27) gelächelt oder gelacht? & $x$ & 1 & 2 & 3 & 4 & 5 & 6 & 7 \\
\hline (28) gejammert oder geschirien? & $\mathrm{x}$ & 1 & 2 & 3 & 4 & 5 & $\overline{6}$ & 7 \\
\hline
\end{tabular}

\section{$\underline{\text { Spielen }}$}

Wie oft hat Ihr Kind in der letzten Woche ...

\begin{tabular}{|c|c|c|c|c|c|c|c|c|}
\hline & \begin{tabular}{|l|l|}
$\begin{array}{l}\text { kam } \\
\text { nicht } \\
\text { vor }\end{array}$ \\
\end{tabular} & nie & $\begin{array}{l}\text { sehr } \\
\text { selten }\end{array}$ & $\begin{array}{l}\text { weniger } \\
\text { als } \\
\text { Hallite } \\
\end{array}$ & \begin{tabular}{|l|} 
Hăltte \\
der \\
Geleg.
\end{tabular} & $\begin{array}{l}\text { mehr } \\
\text { ais } \\
\text { Halfte }\end{array}$ & \begin{tabular}{|l|} 
fist \\
immer
\end{tabular} & immer \\
\hline (29) beim Spielen viel geplappert? & $\mathbf{x}$ & 1 & 2 & 3 & 4 & 5 & 6 & 7 \\
\hline (30) laut gelacht? & $\mathbf{x}$ & 1 & 2 & 3 & 4 & 5 & 6 & 7 \\
\hline $\begin{array}{l}\text { (31) gelächelt oder gelacht, wenn es } \\
\text { gekitzelt wurde? }\end{array}$ & $\mathbf{x}$ & 1 & 2 & 3 & 4 & 5 & 6 & 7 \\
\hline $\begin{array}{l}\text { (32) geweint oder mit Unbehagen reagiert, } \\
\text { wenn es gekitzeit wurde? }\end{array}$ & $\vec{x}$ & 1 & 2 & 3 & 4 & 5 & 6 & 7 \\
\hline
\end{tabular}

Wenn Ihrem Baby etwas weggenommen wurde, womit es gespielt hatte, oder ihm ein Spielzeug runtergefallen ist, wie oft ...

\begin{tabular}{|l|c|c|c|c|c|c|c|c|}
\hline & $\begin{array}{l}\text { kamm } \\
\text { nicht } \\
\text { vor }\end{array}$ & nie & $\begin{array}{l}\text { sehr } \\
\text { selten }\end{array}$ & $\begin{array}{l}\text { wenjger } \\
\text { als } \\
\text { Hălfe }\end{array}$ & $\begin{array}{l}\text { Hălfte } \\
\text { der } \\
\text { Geleg. }\end{array}$ & $\begin{array}{l}\text { mehr } \\
\text { als } \\
\text { Hälfte }\end{array}$ & $\begin{array}{l}\text { fast } \\
\text { inmer }\end{array}$ & immer \\
\hline $\begin{array}{c}\text { (33) schrie oder protestierte es für einen } \\
\text { Moment? }\end{array}$ & $\mathbf{x}$ & $\mathbf{1}$ & $\mathbf{2}$ & $\mathbf{3}$ & $\mathbf{4}$ & $\mathbf{5}$ & $\mathbf{6}$ & $\mathbf{7}$ \\
\hline $\begin{array}{c}\text { (34) schrie oder protestierte es für mehrere } \\
\text { Minuten oder länger? }\end{array}$ & $\mathbf{x}$ & $\mathbf{1}$ & $\mathbf{2}$ & $\mathbf{3}$ & $\mathbf{4}$ & $\mathbf{5}$ & $\mathbf{6}$ & $\mathbf{7}$ \\
\hline
\end{tabular}


Wenn Ihr Baby ein neues Spielzeug bekam, wie oft ...

\begin{tabular}{|l|c|c|c|c|c|c|c|c|}
\hline & $\begin{array}{l}\text { kann } \\
\text { nicht } \\
\text { vor }\end{array}$ & nie & $\begin{array}{l}\text { sehr } \\
\text { selten }\end{array}$ & $\begin{array}{l}\text { weniger } \\
\text { als } \\
\text { Halfee }\end{array}$ & $\begin{array}{l}\text { Hallte } \\
\text { der } \\
\text { Geleg. }\end{array}$ & $\begin{array}{l}\text { mehr } \\
\text { als } \\
\text { Halfte }\end{array}$ & $\begin{array}{l}\text { fast } \\
\text { immer }\end{array}$ & immer \\
\hline $\begin{array}{c}\text { (35) zögerte es beim ersten Anblick des } \\
\text { Spielzeugs? }\end{array}$ & $\mathbf{x}$ & $\mathbf{1}$ & $\mathbf{2}$ & $\mathbf{3}$ & $\mathbf{4}$ & $\mathbf{5}$ & $\mathbf{6}$ & $\mathbf{7}$ \\
\hline (36) wollte es das Spielzeug sofort haben? & $\mathbf{x}$ & $\mathbf{1}$ & $\mathbf{2}$ & $\mathbf{3}$ & $\mathbf{4}$ & $\mathbf{5}$ & $\mathbf{6}$ & $\mathbf{7}$ \\
\hline
\end{tabular}

Wenn das Baby spielerisch in die Höhe gehoben oder geworfen wurde (z.B. „Fliegen“ spielen $0 . a ̈$.), wie oft ...

\begin{tabular}{|l|c|c|c|c|c|c|c|c|}
\hline & $\begin{array}{l}\text { kam } \\
\text { nicht } \\
\text { vor }\end{array}$ & nie & $\begin{array}{l}\text { sehr } \\
\text { selten }\end{array}$ & $\begin{array}{l}\text { weniger } \\
\text { als } \\
\text { Hăife }\end{array}$ & $\begin{array}{l}\text { Halfte } \\
\text { der } \\
\text { Geleg. }\end{array}$ & $\begin{array}{l}\text { mehr } \\
\text { ats } \\
\text { Hallte }\end{array}$ \\
\hline (37) lächelte es? & $\mathbf{x}$ & $\mathbf{1}$ & $\mathbf{2}$ & $\mathbf{3}$ & $\mathbf{4}$ & $\mathbf{5}$ & $\mathbf{6}$ & $\mathbf{7}$ \\
\hline imst & $\mathbf{x}$ & $\mathbf{1}$ & $\mathbf{2}$ & $\mathbf{3}$ & $\mathbf{4}$ & $\mathbf{5}$ & $\mathbf{6}$ & $\mathbf{7}$ \\
\hline
\end{tabular}

Beim Guck-guck-Spiel, wie oft ...

\begin{tabular}{|l|c|c|c|c|c|c|c|c|}
\hline & $\begin{array}{l}\text { kam } \\
\text { nicht } \\
\text { vor }\end{array}$ & nie & $\begin{array}{l}\text { sehr } \\
\text { seiten }\end{array}$ & $\begin{array}{l}\text { weniger } \\
\text { als } \\
\text { Halfe }\end{array}$ & $\begin{array}{l}\text { Haifte } \\
\text { der } \\
\text { Geieg. }\end{array}$ & $\begin{array}{l}\text { mehr } \\
\text { als } \\
\text { Halfe }\end{array}$ & $\begin{array}{l}\text { fast } \\
\text { immer }\end{array}$ & immer \\
\hline (39) lächelte es? & $\mathbf{x}$ & $\mathbf{1}$ & $\mathbf{2}$ & $\mathbf{3}$ & $\mathbf{4}$ & $\mathbf{5}$ & $\mathbf{6}$ & $\mathbf{7}$ \\
\hline (40) lachte es? & $\mathbf{x}$ & $\mathbf{1}$ & $\mathbf{2}$ & $\mathbf{3}$ & $\mathbf{4}$ & $\mathbf{5}$ & $\mathbf{6}$ & $\mathbf{7}$ \\
\hline
\end{tabular}

Wenn Sie mit Ihrem Baby gespielt hatten, dabei aber unterbrochen wurden, wie oft ...

\begin{tabular}{|c|c|c|c|c|c|c|c|c|}
\hline & \begin{tabular}{|l|}
$\begin{array}{l}\text { Karm } \\
\text { nicht } \\
\text { vor }\end{array}$ \\
vor
\end{tabular} & nie & \begin{tabular}{|l|}
$\begin{array}{l}\text { sehr } \\
\text { selten }\end{array}$ \\
\end{tabular} & $\begin{array}{l}\text { weniger } \\
\text { als } \\
\text { Halte } \\
\end{array}$ & \begin{tabular}{|l} 
Hälffe \\
der \\
Geleg.
\end{tabular} & $\begin{array}{l}\text { mehr } \\
\text { als } \\
\text { Halfte }\end{array}$ & $\begin{array}{l}\text { fast } \\
\text { imrner }\end{array}$ & immer \\
\hline (41) machte das Threm Baby nichts aus? & $x$ & 1 & 2 & 3 & 4 & 5 & 6 & 7 \\
\hline (42) weinte es oder zeigte Unbehagen? & $\mathbf{x}$ & 1 & 2 & $\overline{3}$ & 4 & 5 & 6 & 7 \\
\hline
\end{tabular}

\section{$\underline{\text { Tagesaktivität }}$}

Wie oft in der letzten Woche ...

\begin{tabular}{|c|c|c|c|c|c|c|c|c|}
\hline & $\begin{array}{l}\text { karn } \\
\text { nicht } \\
\text { vor }\end{array}$ & nie & $\begin{array}{l}\text { sehr } \\
\text { selen }\end{array}$ & $\begin{array}{l}\text { weniger } \\
\text { als } \\
\text { Hoifte }\end{array}$ & $\begin{array}{l}\text { Falfte } \\
\text { der } \\
\text { Geleg. }\end{array}$ & $\begin{array}{l}\text { mehr } \\
\text { als } \\
\text { Halfe }\end{array}$ & $\begin{array}{l}\text { Gast } \\
\text { irmmer }\end{array}$ & immer \\
\hline $\begin{array}{c}\text { (43) schrie Ihr Baby oder zeigte Unbehagen } \\
\text { bei lauten Geräuschen (Küchen- } \\
\text { maschine, Staubsauger etc.)? }\end{array}$ & $\mathbf{x}$ & $\mathbf{1}$ & $\mathbf{2}$ & $\mathbf{3}$ & $\mathbf{4}$ & $\mathbf{5}$ & $\mathbf{6}$ & $\mathbf{7}$ \\
\hline $\begin{array}{c}\text { (44) protestierte es, wenn es in seiner } \\
\text { Bewegung eingeschränkt wurde } \\
\text { (Kinderstuhl, Laufstall, Autositz)? }\end{array}$ & $\mathbf{x}$ & $\mathbf{1}$ & $\mathbf{2}$ & $\mathbf{3}$ & $\mathbf{4}$ & $\mathbf{5}$ & $\mathbf{6}$ & $\mathbf{7}$ \\
\hline
\end{tabular}




\begin{tabular}{|c|c|c|c|c|c|c|c|c|}
\hline $\begin{array}{c}\text { (45) erschrak das Baby bei einer plötzlichen } \\
\text { Änderung seiner Körperlage, z.B. wenn } \\
\text { es schne!l hochgehoben wurde? }\end{array}$ & $\mathbf{x}$ & $\mathbf{1}$ & $\mathbf{2}$ & $\mathbf{3}$ & $\mathbf{4}$ & $\mathbf{5}$ & $\mathbf{6}$ & $\mathbf{7}$ \\
\hline $\begin{array}{c}\text { (46) erschrak es bei einem lauten oder } \\
\text { plötzlichen Geräusch? }\end{array}$ & $\mathbf{x}$ & $\mathbf{1}$ & $\mathbf{2}$ & $\mathbf{3}$ & $\mathbf{4}$ & $\mathbf{5}$ & $\mathbf{6}$ & $\mathbf{7}$ \\
\hline (47) schrie es, wenn es sich erschreckte? & $\mathbf{x}$ & $\mathbf{1}$ & $\mathbf{2}$ & $\mathbf{3}$ & $\mathbf{4}$ & $\mathbf{5}$ & $\mathbf{6}$ & $\mathbf{7}$ \\
\hline
\end{tabular}

Wenn thr Baby auf dem Arm war, wie oft ...

\begin{tabular}{|l|l|c|c|c|l|l|l|l|}
\hline & $\begin{array}{l}\text { kam } \\
\text { nicht } \\
\text { vor }\end{array}$ & nie & $\begin{array}{l}\text { sehr } \\
\text { selten }\end{array}$ & $\begin{array}{l}\text { weniger } \\
\text { als } \\
\text { Hänthe }\end{array}$ & $\begin{array}{l}\text { Hallte } \\
\text { der } \\
\text { Geleg. }\end{array}$ & $\begin{array}{l}\text { mehr } \\
\text { als } \\
\text { Halfte }\end{array}$ & $\begin{array}{l}\text { fast } \\
\text { immer }\end{array}$ & immer \\
\hline$(48)$ hat es sich heftig bewegt? & $\mathbf{x}$ & $\mathbf{1}$ & $\mathbf{2}$ & $\mathbf{3}$ & $\mathbf{4}$ & $\mathbf{5}$ & $\mathbf{6}$ & $\mathbf{7}$ \\
\hline
\end{tabular}

Wenn das Kind auf den Rücken gelegt wurde, wie oft ....

\begin{tabular}{|c|c|c|c|c|c|c|c|c|}
\hline & $\begin{array}{l}\text { kam } \\
\text { nicht } \\
\text { vor }\end{array}$ & nie & \begin{tabular}{|l} 
sehr \\
seiter
\end{tabular} & \begin{tabular}{|l|} 
wentiger \\
als \\
Hall?.e
\end{tabular} & \begin{tabular}{|l} 
Hälfte \\
der \\
Geieg.
\end{tabular} & \begin{tabular}{|l} 
mehr \\
als \\
Halfee
\end{tabular} & $\begin{array}{l}\text { fast } \\
\text { immer }\end{array}$ & immer \\
\hline (49) protestierte es? & $\mathbf{x}$ & 1 & 2 & 3 & 4 & 5 & 6 & 7 \\
\hline (50) lächelte oder lachte es? & $\mathbf{x}$ & 1 & 2 & 3 & 4 & 5 & 6 & 7 \\
\hline $\begin{array}{l}\text { (51) wedelte es mit den Armen oder } \\
\text { strampelte heftig? }\end{array}$ & $\mathbf{x}$ & 1 & 2 & 3 & 4 & 5 & 6 & 7 \\
\hline (52) hat es sich gedreht und gewunden? & $\mathbf{x}$ & 1 & 2 & 3 & 4 & 5 & 6 & 7 \\
\hline
\end{tabular}

Wenn das Baby etwas wollte, es aber nicht bekam, wie oft ...

\begin{tabular}{|c|c|c|c|c|c|c|c|c|}
\hline & $\begin{array}{l}\begin{array}{l}\text { kamn } \\
\text { nicht } \\
\text { vor }\end{array} \\
\end{array}$ & nie & $\begin{array}{l}\text { sehr } \\
\text { selten }\end{array}$ & \begin{tabular}{|l|l} 
weriger \\
als \\
Halfte \\
\end{tabular} & \begin{tabular}{|l|l} 
Hältte \\
der \\
Geleg.
\end{tabular} & \begin{tabular}{|l} 
mehrs \\
als \\
Hualfte
\end{tabular} & 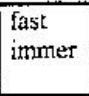 & immer \\
\hline (53) wurde es ärgerlich? & $\mathbf{x}$ & 1 & 2 & 3 & 4 & 5 & 6 & 7 \\
\hline $\begin{array}{l}\text { (54) bekam es einen Wutanfall (Schreien, } \\
\text { rotes Gesicht etc.)? }\end{array}$ & $\mathbf{x}$ & 1 & 2 & 3 & 4 & 5 & 6 & 7 \\
\hline
\end{tabular}

Wenn das Kind in einen Kinderstuhl oder Autositz gesetzt wurde, wie oft ...

\begin{tabular}{|c|c|c|c|c|c|c|c|c|}
\hline & \begin{tabular}{|l}
$\begin{array}{l}\text { kan } \\
\text { nicht } \\
\text { vor }\end{array}$ \\
\end{tabular} & nie & $\begin{array}{l}\text { sehr } \\
\text { selten }\end{array}$ & 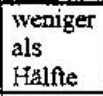 & \begin{tabular}{|l} 
Hillter \\
der \\
Geleg.
\end{tabular} & $\begin{array}{l}\text { mehr } \\
\text { als } \\
\text { Halfte }\end{array}$ & \begin{tabular}{|l|} 
fast \\
immer
\end{tabular} & immer \\
\hline $\begin{array}{l}\text { (55) wedelte es mit den Armen und } \\
\text { strampelte heftig? }\end{array}$ & $\mathbf{x}$ & 1 & 2 & 3 & 4 & 5 & 6 & 7 \\
\hline (56) hat es sich gedreht und gewunden? & $\mathbf{x}$ & 1 & 2 & 3 & 4 & 5 & 6 & 7 \\
\hline (57) lag oder $\mathrm{sa} ß$ es ruhig? & $\mathbf{x}$ & 1 & 2 & 3 & 4 & 5 & 6 & 7 \\
\hline (58) zeigte es anfangs Unbehagen? & $\mathbf{x}$ & 1 & 2 & 3 & 4 & 5 & 6 & 7 \\
\hline
\end{tabular}


Im Autositz, Kinderstuhl oder Babywippe, wie oft ...

\begin{tabular}{|l|c|c|c|c|l|l|l|l|}
\hline & $\begin{array}{l}\text { kamn } \\
\text { richt } \\
\text { vor }\end{array}$ & nie & $\begin{array}{l}\text { sehr } \\
\text { selten }\end{array}$ & $\begin{array}{l}\text { weniger } \\
\text { als } \\
\text { Häne }\end{array}$ & $\begin{array}{l}\text { Hallte } \\
\text { der } \\
\text { Geleg. }\end{array}$ & $\begin{array}{l}\text { mehr } \\
\text { als } \\
\text { Hallte }\end{array}$ & $\begin{array}{l}\text { fast } \\
\text { inmer }\end{array}$ & immer \\
\hline (59) plapperte Thr Baby viel? & $\mathbf{x}$ & $\mathbf{1}$ & $\mathbf{2}$ & $\mathbf{3}$ & $\mathbf{4}$ & $\mathbf{5}$ & $\mathbf{6}$ & $\mathbf{7}$ \\
\hline
\end{tabular}

Wenn Sie wegwaren, dann wiederkamen, und thr Kind wach war, wie oft ...

\begin{tabular}{|l|l|l|l|l|l|l|l|c|}
\hline & $\begin{array}{l}\text { kam } \\
\text { nicht } \\
\text { vor }\end{array}$ & nie & $\begin{array}{l}\text { sehr } \\
\text { seiten }\end{array}$ & $\begin{array}{l}\text { weniger } \\
\text { als } \\
\text { Halfie }\end{array}$ & $\begin{array}{l}\text { Halthe } \\
\text { der } \\
\text { Geieg. }\end{array}$ & $\begin{array}{l}\text { mehr } \\
\text { als } \\
\text { Hallte }\end{array}$ & $\begin{array}{l}\text { fast } \\
\text { immer }\end{array}$ & immer \\
\hline (60) lächelte oder lachte es? & $\mathbf{X}$ & $\mathbf{1}$ & $\mathbf{2}$ & $\mathbf{3}$ & $\mathbf{4}$ & $\mathbf{5}$ & $\mathbf{6}$ & $\mathbf{7}$ \\
\hline
\end{tabular}

Wenn Sie Ihr Baby ablegten (Bett, Sofa, Spieldecke etc.), wie oft ...

\begin{tabular}{|l|c|c|c|c|c|c|c|c|}
\hline & $\begin{array}{l}\text { karn } \\
\text { nicht } \\
\text { vor }\end{array}$ & nie & $\begin{array}{l}\text { sehr } \\
\text { selten }\end{array}$ & $\begin{array}{l}\text { weniger } \\
\text { als } \\
\text { Hallte }\end{array}$ & $\begin{array}{l}\text { सallte } \\
\text { der } \\
\text { Geieg. }\end{array}$ & $\begin{array}{l}\text { mehr } \\
\text { als } \\
\text { Hallte }\end{array}$ & $\begin{array}{l}\text { fast } \\
\text { inmer }\end{array}$ & irmer \\
\hline (61) quengelte es bald? & $\mathbf{X}$ & $\mathbf{1}$ & $\mathbf{2}$ & $\mathbf{3}$ & $\mathbf{4}$ & $\mathbf{5}$ & $\mathbf{6}$ & $\mathbf{7}$ \\
\hline (62) war es für längere Zeit zufrieden? & $\mathbf{X}$ & $\mathbf{1}$ & $\mathbf{2}$ & $\mathbf{3}$ & $\mathbf{4}$ & $\mathbf{5}$ & $\mathbf{6}$ & $\mathbf{7}$ \\
\hline
\end{tabular}

Wenn Ihr Baby mit einer fremden Person zusammenkam, wie oft ...

\begin{tabular}{|c|c|c|c|c|c|c|c|c|}
\hline & $\begin{array}{l}\text { kam } \\
\text { nicht } \\
\text { vor }\end{array}$ & nie & \begin{tabular}{|l} 
sehr \\
selten
\end{tabular} & $\begin{array}{l}\text { weniger } \\
\text { als } \\
\text { Halfte } \\
\end{array}$ & \begin{tabular}{|l} 
Hällte \\
der \\
Geleg.
\end{tabular} & $\begin{array}{l}\text { mehr } \\
\text { ais } \\
\text { Hullte }\end{array}$ & \begin{tabular}{|l|} 
Fast \\
intmer
\end{tabular} & immer \\
\hline (63) wollte es nur bei den Eltern bleiben? & $\mathbf{x}$ & 1 & 2 & 3 & 4 & 5 & 6 & 7 \\
\hline $\begin{array}{l}\text { (64) wollte es mit dem Fremden keinen } \\
\text { Kontakt aufnehmen? }\end{array}$ & $\mathbf{x}$ & 1 & 2 & 3 & 4 & 5 & 6 & 7 \\
\hline $\begin{array}{l}\text { (65) wendete es sich sofort dem Fremden } \\
\text { zu? }\end{array}$ & $\mathbf{x}$ & 1 & 2 & 3 & 4 & 5 & 6 & 7 \\
\hline
\end{tabular}

Wenn Sie mit Ihrem Baby in eine fremde Umgebung kamen, wie oft ...

\begin{tabular}{|l|c|c|c|c|c|c|c|c|}
\hline & $\begin{array}{l}\text { kam } \\
\text { nicht } \\
\text { vor }\end{array}$ & nie & $\begin{array}{l}\text { sehr } \\
\text { selten }\end{array}$ & $\begin{array}{l}\text { weniger } \\
\text { als } \\
\text { Hälfte }\end{array}$ & $\begin{array}{l}\text { Hälte } \\
\text { der } \\
\text { Gelez. }\end{array}$ & $\begin{array}{l}\text { mehr } \\
\text { als } \\
\text { Hälthe }\end{array}$ & $\begin{array}{l}\text { fast } \\
\text { inmer }\end{array}$ & immer \\
\hline (66) reagierte es sofort interessiert? & $\mathbf{X}$ & $\mathbf{1}$ & $\mathbf{2}$ & $\mathbf{3}$ & $\mathbf{4}$ & $\mathbf{5}$ & $\mathbf{6}$ & $\mathbf{7}$ \\
\hline $\begin{array}{c}\text { (67) reagierte es mit Zurückhaltung oder } \\
\text { Unbehagen? }\end{array}$ & $\mathbf{X}$ & $\mathbf{1}$ & $\mathbf{2}$ & $\mathbf{3}$ & $\mathbf{4}$ & $\mathbf{5}$ & $\mathbf{6}$ & $\mathbf{7}$ \\
\hline
\end{tabular}


Wenn Ihr Baby in den letzten 2 Wochen quengelte oder schrie und Sie es beruhigen wollten, wie oft ließ sich Ihr Baby schnell beruhigen durch:

\begin{tabular}{|c|c|c|c|c|c|c|c|c|}
\hline & \begin{tabular}{|l|} 
kam \\
richt \\
oor \\
\end{tabular} & nie & \begin{tabular}{|l|} 
sehr \\
seiten
\end{tabular} & \begin{tabular}{|l} 
weniger \\
als \\
itulfe
\end{tabular} & $\begin{array}{l}\text { Hülfte } \\
\text { der } \\
\text { Geleg. }\end{array}$ & \begin{tabular}{|l} 
methr \\
als \\
Halfe
\end{tabular} & \begin{tabular}{|l|} 
tast \\
immer
\end{tabular} & immer \\
\hline (68) Schaukein und Wiegen & $\mathbf{x}$ & 1 & 2 & 3 & 4 & 5 & 6 & 7 \\
\hline (69) Sprechen und Singen & $\mathbf{x}$ & 1 & 2 & 3 & 4 & 5 & 6 & 7 \\
\hline (70) Verändem der Körperlage & $x$ & 1 & 2 & 3 & 4 & 5 & 6 & 7 \\
\hline (71) Schnuller geben & $\mathbf{x}$ & 1 & 2 & 3 & 4 & 5 & 6 & 7 \\
\hline (72) Etwas zu Essen geben & $\mathbf{x}$ & 1 & 2 & 3 & 4 & 5 & 6 & 7 \\
\hline (73) Streicheln & $\mathbf{x}$ & 1 & 2 & 3 & 4 & 5 & 6 & 7 \\
\hline (74) Etwas Interessantes zeigen & $\mathbf{x}$ & 1 & 2 & 3 & 4 & 5 & 6 & $\overline{7}$ \\
\hline (75) Ein Spielzeug geben & $\mathbf{x}$ & 1 & 2 & 3 & 4 & 5 & 6 & 7 \\
\hline (76) Auf den Anm nehmen & $x$ & 1 & 2 & 3 & 4 & 5 & 6 & 7 \\
\hline (77) Auf dem Arm umhertragen & $\mathbf{x}$ & 1 & 2 & 3 & 4 & 5 & 6 & 7 \\
\hline 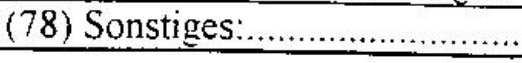 & & & & & & & & \\
\hline
\end{tabular}


Fragebogenskala „Depressivität“ und „Überfürsorge aus Angst“ aus dem EMKK von Engfer

Die Aussagen in diesem Fragebogen geben Erfahrungen wieder, die Sie im Umgang mit Threm Kind machen können oder die allgemeiner mit Kindern zu tun haben.

Bitte kreuzen Sie jeweils die Antwortmoglichkeit an, die Ihrer Meinung nach am besten auf Sie zutrifft.

Bitte lassen Sie keinen Satz aus!

\begin{tabular}{|c|c|c|c|c|}
\hline & $\begin{array}{c}\text { trifft } \\
\text { sehr zu }\end{array}$ & trifft zu & $\begin{array}{l}\text { trifft } \\
\text { eher } \\
\text { nicht zu }\end{array}$ & $\begin{array}{l}\text { trifft } \\
\text { gar } \\
\text { nicht zu }\end{array}$ \\
\hline $\begin{array}{l}\text { 1. Es macht mich sehr kribbelig, wenn } \\
\text { mein Kind nicht richtig trinkt. }\end{array}$ & & & & \\
\hline $\begin{array}{l}\text { 2. Ich bin stäudig in Sorge, daß } \\
\text { meinem Kind was zustoßen könnte. }\end{array}$ & & & & \\
\hline $\begin{array}{l}\text { 3. Seit der Entbindung bin ich viel } \\
\text { nervöser geworden. }\end{array}$ & & & & \\
\hline $\begin{array}{l}\text { 4. Ich werde den Gedanken nicht los, } \\
\text { daß mein Kind doch krank sein könnte. }\end{array}$ & & & & \\
\hline $\begin{array}{l}\text { 5. Seit das Kind da ist, fülle ich mich } \\
\text { innerlich angespannt. }\end{array}$ & & & & \\
\hline $\begin{array}{l}\text { 6. Es ist für mich unvorstellbar, mein } \\
\text { Kind auch nur für Minuten allein zu } \\
\text { lassen. }\end{array}$ & & & & \\
\hline $\begin{array}{l}\text { 7. Im Grunde weiß ich nicht, ob ich } \\
\text { was bei meinem Kind falsch mache. }\end{array}$ & & & & \\
\hline $\begin{array}{l}\text { 8. Manchmal würde ich am liebsten } \\
\text { nur noch schlafen und alle Probieme } \\
\text { vergessen. }\end{array}$ & & & & \\
\hline $\begin{array}{l}\text { 9. lu der letzten Zeit weine ich viel } \\
\text { häufiger. }\end{array}$ & & & & \\
\hline $\begin{array}{l}\text { 10. Ich kömnte mein Kind nie einem } \\
\text { Babysitter überlassen, aus Sorge, daß } \\
\text { er das Kind nicht richtig behandelt. }\end{array}$ & & & & \\
\hline $\begin{array}{l}\text { 11. Ich frage mich standig, ob ich bei } \\
\text { meinem Kind alles richtig mache. }\end{array}$ & & & & \\
\hline $\begin{array}{l}\text { 12. Im Grunde fühie ich mich mit } \\
\text { meinen Problemen ganz allein } \\
\text { gelassen, weil sich niemand wirklich } \\
\text { um mich kümmert. }\end{array}$ & & & & \\
\hline $\begin{array}{l}\text { 13. Andere Leute lasse ich an mein } \\
\text { Kind nicht ran, weil man nie weiß was } \\
\text { passieren könnte. }\end{array}$ & & & & \\
\hline $\begin{array}{l}\text { 14. Bei meinem Kind habe ich das } \\
\text { Gefuilhl. daB ich etwas falsch mache. }\end{array}$ & & & & \\
\hline
\end{tabular}




\begin{tabular}{|c|c|c|c|c|}
\hline & $\begin{array}{c}\text { trifft } \\
\text { sehr zu }\end{array}$ & trifft zu & $\begin{array}{l}\text { trifft } \\
\text { eher } \\
\text { nicht zu }\end{array}$ & $\begin{array}{l}\text { trifft } \\
\text { gar } \\
\text { nicht zu }\end{array}$ \\
\hline $\begin{array}{l}\text { 15. Manchmal labe ich das Gefuhi, } \\
\text { daß mich mein Kind nicht mag. }\end{array}$ & & & & \\
\hline $\begin{array}{l}\text { 16. Ich schaue nachts manchimal nach, } \\
\text { ob mein Kind auch wirkfich noch } \\
\text { atmet. }\end{array}$ & & & & \\
\hline $\begin{array}{l}\text { 17. Niemand scheint daran zu denken, } \\
\text { daß ich auch Liebe und Hilfe brauche. }\end{array}$ & & & & \\
\hline 18. Ich fühle mich recht erschöpft. & & & & \\
\hline $\begin{array}{l}\text { 19. Bei Krankheiten des Kindes } \\
\text { überfällt mich plötzlich der Gedanke, } \\
\text { daß mir das Kind wieder genommen } \\
\text { werden könnte. }\end{array}$ & & & & \\
\hline $\begin{array}{l}\text { 20. Ich finde es schwierig zu wissen, } \\
\text { was meinem Kind fehlt, wenn es } \\
\text { quentrelt. }\end{array}$ & & & & \\
\hline $\begin{array}{l}\text { 2l. Es macht mir große Mühe, } \\
\text { überhaupt noch Pläne zu machen und } \\
\text { in die Tat umzusetzen. }\end{array}$ & & & & \\
\hline $\begin{array}{l}\text { 22. Manchmal kann ich nachts nicht } \\
\text { schlafen, weil ich mir vorstelle, } \\
\text { meinem Kind kömnte etwas zustoßen. }\end{array}$ & & & & \\
\hline $\begin{array}{l}\text { 23. Ich habe manchunal Angst, daß ich } \\
\text { meinem Kind wehtue. }\end{array}$ & & & & \\
\hline $\begin{array}{l}\text { 24. Es kränkt mich, wenn mein Kind } \\
\text { nicht trinken mag. }\end{array}$ & & & & \\
\hline $\begin{array}{l}\text { 25. Ich fühle mich oft am Ende meiner } \\
\text { Kraft. }\end{array}$ & & & & \\
\hline $\begin{array}{l}\text { 26. Ich frage mich manchmal, ob ich } \\
\text { meinem Kind beim Trockenlegen nicht } \\
\text { selur wehtue. }\end{array}$ & & & & \\
\hline $\begin{array}{l}\text { 27. Mein Alltag erscheint mir } \\
\text { vollkommen zerstückelt. }\end{array}$ & & & & \\
\hline $\begin{array}{l}\text { 28. Manctinal überfällt mich der } \\
\text { Gedanke, daB ich mein Kind verletzen } \\
\text { könnte. }\end{array}$ & & & & \\
\hline $\begin{array}{l}\text { 29. Ich werde den Gedanken richt los, } \\
\text { daB ich im Grunde keine gute Mufter } \\
\text { bin. }\end{array}$ & & & & \\
\hline
\end{tabular}




\begin{tabular}{|c|c|c|c|c|}
\hline & $\begin{array}{c}\text { trifft } \\
\text { sehr zu }\end{array}$ & trifft za & $\begin{array}{c}\text { trifft } \\
\text { eher } \\
\text { nicht zu }\end{array}$ & $\begin{array}{c}\text { trifft } \\
\text { gar } \\
\text { nicht zu }\end{array}$ \\
\hline $\begin{array}{l}\text { 30. Ich darf nicht zulassen, daß das } \\
\text { Kind so wird wie ich. }\end{array}$ & & & & \\
\hline $\begin{array}{l}\text { 31. Ich fühle mich enttäuscht, wenn } \\
\text { mein Kind meine Zärtlichkeiten } \\
\text { abwehrt. }\end{array}$ & & & & \\
\hline $\begin{array}{l}\text { 32. Ich ärgere mich, wenn man mich } \\
\text { für eine schlechte Mutter hält, bloß } \\
\text { weil das Kind schreit. }\end{array}$ & & & & \\
\hline
\end{tabular}


Fragebogenskala „Trait-Angst“ aus dem STAI von Laux et al.

\section{Fragebogen zur Selbstbeschreibung}

STAI-G Form $\times 2$

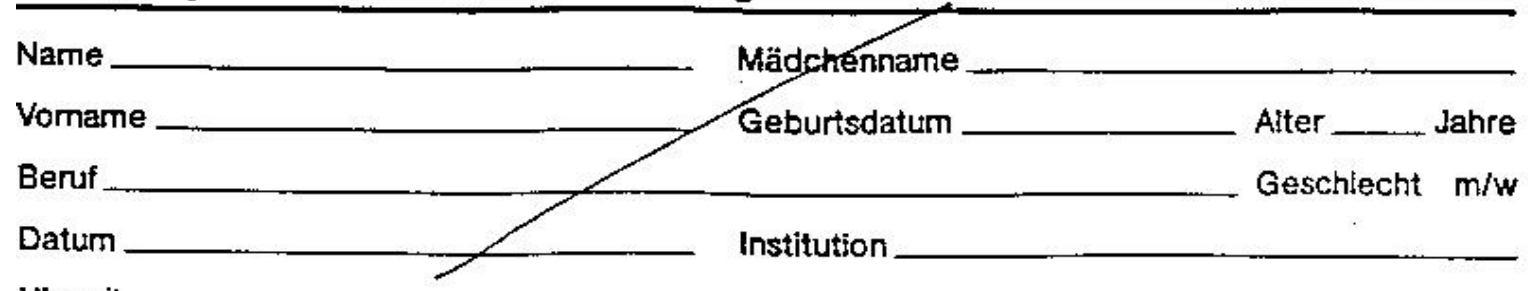

Uhrzeit

\begin{tabular}{|c|c|c|c|c|}
\hline $\begin{array}{l}\text { Anleitung: Im foigenden Fragebogen finden Sie eine Reihe von Feststeliungen, } \\
\text { mit denen man sich selbst beschreiben kann. Bitte lesen Sie jede Feststeliung } \\
\text { durch und wählen Sie aus den vier Antworten diejenige aus, die angibt, wie Sie } \\
\text { sich im allgemeinen fühlen. Kreuzen Sie bitte beijecierFeststellung die Zahl lunter } \\
\text { der von lhnen gewählten Antwort an. Es gibt keine richtigen oder falschen Ant- } \\
\text { worten. Überlegen Sie bitte nicht lange und denken Sie daran, diejenige Antwort } \\
\text { auszuwähien, die am besten beschreibt, wie Sie sich im allgemeinen fühlen. }\end{array}$ & $\begin{array}{l}\frac{\omega}{z} \\
\frac{5}{2} \\
\underline{x}\end{array}$ & $\begin{array}{l}\frac{1}{\frac{1}{2}} \\
\frac{1}{d} \\
\frac{2}{2} \\
\frac{1}{2}\end{array}$ & $\frac{5}{0}$ & $\frac{\frac{r}{4}}{\frac{1}{2}}$ \\
\hline 21. Ich bin vergnügt & 1 & 2 & 3 & 4 \\
\hline 22 lch werde schnelt müde & 1 & 2 & 3 & 4 \\
\hline 23. Mir ist zum Weinen zumute & 1 & 2 & 3 & 4 \\
\hline 24. Ich glaube, mir geht es schlechter als anderen Leuten & 1 & 2 & 3 & 4 \\
\hline $\begin{array}{l}\text { 25. Ich verpasse günstige Geiegenheiten, weil ich mich nicht schnell genug } \\
\text { entscheiden kann }\end{array}$ & 1 & 2 & 3 & 4 \\
\hline 26. Ich fühle mich ausgeruht & 1 & 2 & 3 & 4 \\
\hline 27. Ich bin ruhig und gelassen & 1 & 2 & 3 & 4 \\
\hline 28. lch głaube, daB mir meine Schwierigkeiten über den Kopf wachsen & 1 & 2 & 3 & 4 \\
\hline 29. Ich mache mir zuviel Gedanken ủber unwichtige Dinge & 1 & 2 & 3 & 4 \\
\hline 30. Ich bin glücklich & 1 & 2 & 3 & 4 \\
\hline 31. Ich neige dazu. alles schwor zu nehmen & $t$ & 2 & 3 & 4 \\
\hline 32. Mir fehlt es an Selbstvertrauen & 1 & 2 & 3 & 4 \\
\hline 33. tch tithle mitch geborgen & 1 & 2 & 3 & 4 \\
\hline 34. Ich mache mir Sorgen über mögliches Milggeschick & 1 & 2 & 3 & 4 \\
\hline 35. Ich fithle mich niedergeschtagen & 1 & 2 & 3 & 4 \\
\hline 36. lch bin zufrieden & 1 & 2 & 3 & 4 \\
\hline 37. Unwichtige Gedanken gehen mir durch den Kopf und bedrücken mich & 1 & 2 & 3 & 4 \\
\hline 38. Enttäuschungen nehme ich so schwer, daß ich sie nicht vergessen kann & $i$ & 2 & 3 & 4 \\
\hline 39. Ich bin ausgegtichen & 1 & 2 & 3 & 4 \\
\hline $\begin{array}{l}\text { 40. lch werde nervós und unruhig, wenn ich an meine dereitigen Angelegen- } \\
\text { heiten denke }\end{array}$ & 1 & 2 & 3 & 4 \\
\hline
\end{tabular}


Fragebogenskala „Hoffnungslosigkeit“ von Krampen

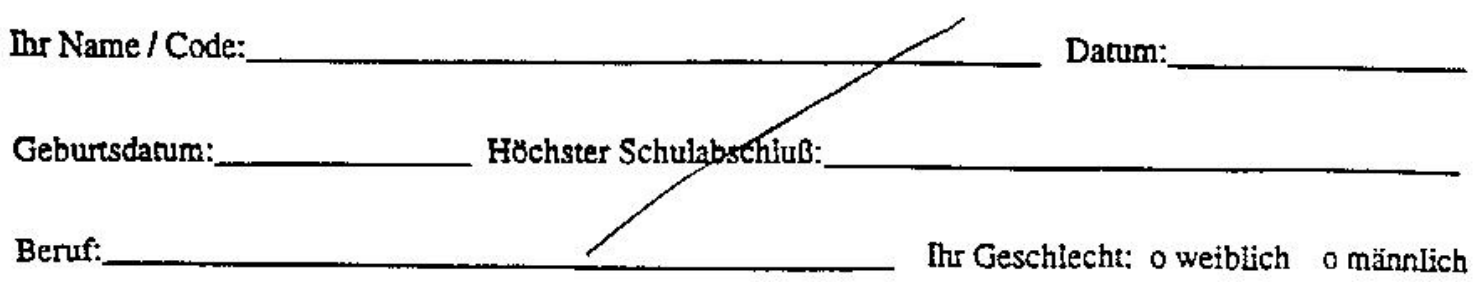

\section{ANLEITUNG}

Geben Sie bitte bei den folgenden Aussagen an, ob sie für Sie persönlich zutreffen oder nicht. Tun Sie dies, indem Sie den entsprechenden Kreis für RICHTTG oder FALSCH ankreuzen.

Hier ist ein Beispiel für die Beantwortung der Aussagen:

\section{FALSCH RICHTG}

"Ich bin ein zunickhaitender Mensch." $\quad 0 \quad 0$

Ist diese Aussage für Sie falsch, so durchkreuzen Sie bitte den Kreis unter "FALSCH". Ist diese Aussage für Sie richtig. so dưrchkreuzen Sie bitte den Kreis untêr "RICHTIG".

Bitte bearbeiten Sie alle Aussagen der Reihe nach, ohne eine auszulassen. Einige Aussagen haben einen ăhnlichen Wortaut oder Sinn. Bitte nehmen Sie auch zu diesen Aussagen Steilung. Es geht bei allen Aussagen um Thre ganz persönliche Sichtweise.

\section{Hier bitte nichts ausftillen!}

\section{Auswertung:}

H-S-Rohwer: PR-Wer: T-Wert: 
Im folgenden werden Sie gebeten, zu einigen Aussagen Steilung zu nehmen. Markieren Sie bitte jeweils die Antwort, die Ihrer persönlichen Meinung am besten entspricht.

01. Ich blicke mit Optimismus und Begeisterung in die Zukunft.

02. Häufig möchte ich alles hinschmeißen, weil ich es doch nicht besser machen kann.

03. Wenn eirmal alles schiefläuft, geht es mir besser, wenn ich daran denke, daß es ja auch wieder aufwärsggehen wird.

$0 \quad 0$

04. Ich karn mir nicht vorstellen, wie mein Leben in zehn Jahren aussehen wird.

05. Ich habe genug Zeit, um die Sachen, die mir Spaß machen, zu tun.

06. Das, womit ich mich im Moment am meisten beschäftige, wird mir in der Zukunft Nutzen bringen.

07. Die Zukunft liegt für mich im Dunkeln.

08. Ich erwarte, in meinem Leben mehr Schönes zu erleben als der durchschnittiche Mensch.

09. Ich kriege eintach keine richtigen Chancen im Leber.

10. Meine Erfahrungen sind eine gute Vorbereitung für künftige Probleme.

0

11. Alles, was ich im Moment so vor mir liegen sehe. ist eher unschön ais schŏn und angenehm.

Ich gaaube nicht, daß ich jemais das in Leben bekomme, was ich mir in

Wahrheit wïnsche.

13. Ich glaube. daß ich in kitnftigen Zeiten glücklicher sein werde als heute.

14. Die Dinge laufen einfach nicht so, wie ich es geme hătte.

0

15. Ich setze große Hoffnungen in die Zukunft.

$\begin{array}{ll}0 & 0 \\ 0 & 0 \\ 0 & 0 \\ 0 & 0\end{array}$

16. Ich bexomme einfach nie das, was ich will: es ist aiso Unsinn. Uberhaupt noch etwas zu wollen.

17. Es ist sehr unwahrscheinlich, daß das Leben mir noch Befriedigung und Freude bringt.

18. Die Zukunft erscheint mir sehr unsicher.

19. Das Leben wird mir noch viel mehr schöne Zeiten bringen ais schlechte.

20. Es niltzt nichts, etwas anzustreben. das ich geme hätte, da ich es wahsscheinlich ja doch nicht erreiche. 


\section{Danksagung}

An dieser Stelle möchte ich mich sehr bei meiner Betreuerin Frau PD Dr. Ursula Pauli-Pott für ihre Betreuung, ihre Gesprächsbereitschaft, ihre Hilfe bei der statistischen Auswertung der Daten und der sorgfältigen Durchsicht dieser Arbeit bedanken. Des Weiteren möchte ich mich bei Frau Hildegard Mathea bedanken, die mich ebenfalls durch wiederholte Durchsicht der Arbeit tatkräftig unterstützt hat.

Weiterhin wäre diese Arbeit nicht ohne die Mitarbeit und das Engagement der vielen Mütter zustande gekommen, welche uns die Daten über Ihre Säuglinge zur Verfügung gestellt haben. An dieser Stelle ein großes Dankeschön.

Zum Abschluss danke ich Frau Professorin Dr. R. Deinzer, Herrn Professor Dr. D. Beckmann und Herrn Professor Dr. U. Gieler, welche mir als Leiterin/ Leiter der Abteilung für Medizinische Psychologie der Universität Giessen diese Promotionsarbeit ermöglicht haben. 


\section{Lebenslauf}

Name:

Anschrift:

Geburtsdatum/-ort:

Staatsangehörigkeit:

Familienstand:

Schulausbildung/-abschluss:

Wehr-/ Ersatzdienste:

Hochschulstudium:

Berufliche Tätigkeit:
Andreas Heitkamp

Josefstr. 1, 83278 Traunstein

6. Mai 1978 in Remscheid

deutsch

ledig

1984 bis 1988 St. Katharina Grundschule Hückeswagen

1988 bis 1997 Erzbischhöfliches St. Angela Gymnasium Wipperfürth, Zeugnis der allgemeinen Hochschulreife

1997- 1998 Zivildienst beim DRK Wipperfürth

WS 1998/99 Studium Wirtschaftsingenieur an der Universität Siegen

SS 1999 - WS 2005/ 06 Studium der Humanmedizin an der Justus-Liebig-Universität in Gießen Abschluss: Staatsexamen

seit März 2006 Assistenzarzt in der Abteilung Anästhesiologie, operative Intensivmedizin und Schmerztherapie am Klinikum Traunstein 


\section{Erklärung}

„Ich erkläre: Ich habe die vorgelegte Dissertation selbständig, ohne unerlaubte fremde Hilfe und nur mit den Hilfen angefertigt, die ich in der Dissertation angegeben habe. Alle Textstellen, die wörtlich oder sinngemäß aus veröffentlichten oder nicht veröffentlichten Schriften entnommen sind, und alle Angaben, die auf mündlichen Auskünften beruhen, sind als solche kenntlich gemacht. Bei den von mir durchgeführten und in der Dissertation erwähnten Untersuchungen habe ich die Grundsätze guter wissenschaftlicher Praxis, wie sie in der „Satzung der Justus-Liebig- Universität GieBen zur Sicherung guter wissenschaftlicher Praxis" niedergelegt sind, eingehalten." 